\title{
Kognitiv-behaviorale und tiefenpsychologisch fundierte Therapie der Generalisierten Angst:
}

\section{Ein Therapieprozessvergleich}

\author{
Dissertation \\ zur Erlangung des Doktorgrades \\ der Mathematisch-Naturwissenschaftlichen Fakultäten \\ der Georg-August-Universität zu Göttingen
}

vorgelegt von

Norina Hofmann

aus Dresden

Göttingen 2007 
D 7

Referentin: Prof. Dr. B. Kröner-Herwig Korreferent: Prof. Dr. E. Leibing

Tag der mündlichen Prüfung: 23.01.2008 


\section{Danksagung}

Bedanken möchte ich mich bei Prof. Dr. Kröner-Herwig, Prof. Dr. Leibing und Prof. Dr. Leichsenring für die vielen wertvollen Tipps in den verschiedenen Phasen dieser Arbeit. Sie haben die Rahmenbedingungen für die Erstellung dieser Arbeit geschaffen und sie so überhaupt erst ermöglicht.

Außerdem danke ich meiner Kollegin Simone Salzer für die geduldige Bearbeitung meiner Anliegen.

Bei der Erhebung der Daten unterstützten mich Hedda Enkemann und Katja Jamrozinski; auch bei ihnen und allen Patienten und den behandelnden Therapeuten möchte ich mich bedanken.

Mein besonderer Dank gilt P. Goldmann für seine Unterstützung, Geduld und Ermutigung. 


\section{Inhaltsverzeichnis}

i. Verzeichnis der Tabellen

ii. Verzeichnis der Abbildungen

0. Zusammenfassung 1

I. Einleitung 2

II. Theoretischer und empirischer Hintergrund 7

1. Theoretische und empirische Fundierung der kognitiv-behavioralen und der $\begin{array}{ll}\text { psychodynamischen Psychotherapie } & 7\end{array}$

1.1 Kognitiv-behaviorale Ansätze $\quad 7$

1.1.1 Grundprinzipien und historische Entwicklung 7

$\begin{array}{ll}\text { 1.1.2 Stand der Outcome-Forschung } & 10\end{array}$

1.1.3 Wirkfaktoren der kognitiv-behavioralen Therapie 10

$\begin{array}{ll}1.2 \text { Psychodynamische Ansätze } & 11\end{array}$

1.2.1 Historische Entwicklung und Grundprinzipien 11

1.2.2 Unterscheidung von tiefenpsychologisch fundierter und analytischer $\begin{array}{ll}\text { Psychotherapie } & 15\end{array}$

1.2.3 Stand der psychodynamischen Outcome-Forschung 17

$\begin{array}{ll}\text { 1.2.4 Wirkfaktoren psychodynamischer Psychotherapie } & 17\end{array}$

1.3 Vergleich der Störungskonzepte von psychodynamischen und kognitiv$\begin{array}{ll}\text { behavioralen Therapieansätzen } & 18\end{array}$

2. Psychotherapie und Psychotherapieforschung 19

2.1 Psychotherapie-Outcome versus Psychotherapie-Prozess 19

$\begin{array}{ll}2.2 \text { Psychotherapieforschung } & 20\end{array}$

3. Psychotherapieprozessforschung 22

3.1 Methodische Anforderungen an die Psychotherapieprozessforschung 22

3.2 Das Psychotherapie Prozess Q-Sort (Jones, 2000) 26

3.3 Befunde aus der Prozessforschung mit dem PQS 28

3.4 Bewertung des PQS 34

3.5 Erste allgemeine Befunde aus der Psychotherapieprozessforschung:

Unterscheidung von unspezifischen und spezifischen Wirkfaktoren 36

3.5.1 Theoretische Annahmen und empirische Befunde zu unspezifischen $\begin{array}{ll}\text { Wirkfaktoren } & 36\end{array}$ 
3.5.2 Vergleich des Einflusses von unspezifischen und spezifischen Wirkfaktoren

3.6 Vergleich der unspezifischen und spezifischen Prozessmerkmale in psychodynamischen und kognitiv-behavioralen Therapien

4. Die Generalisierte Angststörung 41

4.1 Das Störungsbild 41

4.2 Entstehung und Aufrechterhaltung einer Generalisierten Angststörung 44

4.3 Therapie der Generalisierten Angststörung 47

$\begin{array}{ll}\text { 4.3.1 Psychopharmako-Therapie } & 47\end{array}$

$\begin{array}{ll}\text { 4.3.2 Psychotherapie } & 47\end{array}$

5. Fragestellung der vorliegenden Arbeit 54

$\begin{array}{ll}\text { III. Methode } & 60\end{array}$

$\begin{array}{ll}\text { 1. Beschreibung der Stichprobe } & 60\end{array}$

$\begin{array}{ll}\text { 1.1 Patienten } & 60\end{array}$

$\begin{array}{ll}1.2 \text { Therapeuten } & 63\end{array}$

2. Messinstrumente zur Erfassung diagnose- und outcome-relevanter Maße 64

$\begin{array}{ll}2.1 \text { Fremdbeurteilungsmaße } & 64\end{array}$

2.2 Selbstbeurteilungsmaße 66

3. Erfassung des Therapieprozesses: Das Psychotherapy Process Q-Sort (PQS) 69

4. Hypothesen und Fragestellungen und statistische Verfahren zu ihrer Auswertung 77

IV. Ergebnisse $\quad 82$

1. Vergleich der Intraklassenkorrelation zwischen den beiden Behandlungsgruppen 82

2. Vergleich der PQS-Ratings für die 10. und 18. Sitzung: Überprüfung der Hypothese 1

3. Vergleich der PQS-Ratings der psychodynamisch und der kognitiv-behavioral behandelten Patienten: Überprüfung der Hypothese 2

4. Übereinstimmung der implementierten Therapien mit den jeweiligen Prototypen: $\begin{array}{ll}\text { Überprüfung der Hypothese } 3 & 90\end{array}$

5. Zusammenhang zwischen der Stärke der Orientierung am zugehörigen Prototyp und dem Therapieoutcome: Überprüfung der Fragestellung A 93

6. PQS-Items als Prädiktoren des Therapieerfolgs: Überprüfung der Fragestellung B 94

V. Diskussion 99

1. Zur Reliabilität der Prozessbeurteilungen der Rater 99

2. Zum multiplen Testen in der vorliegenden Untersuchung 100 


\section{Inhaltsverzeichnis}

3. Der Therapieprozess in der 10. und 18. Sitzung: Gibt es Unterschiede? 101

4. Der Therapieprozess in der psychodynamischen und kognitiv-behavioralen Therapie: Gibt es Unterschiede?

5. Übereinstimmung der implementierten Therapien mit ihren jeweiligen Prototypen

6. Zusammenhang zwischen der Orientierung an einem Prototyp und dem Outcome

7. Therapieprozessmerkmale als Prädiktoren des Outcomes

8. Grenzen der Untersuchung

9. Einordnung der Ergebnisse und Ausblick 


\section{i. Verzeichnis der Tabellen}

Tabelle 1: Rangreihe der Q-Items anhand der Faktorwerte „psychodynamische Technik“.

Tabelle 2: Rangreihe der Q-Items anhand der Faktorwerte „kognitiv-behaviorale Technik“.

Tabelle 3: Übersicht über unspezifische Therapieprozesse nach Lambert und Ogles.

Tabelle 4: Soziodemographische Daten der Untersuchungsstichprobe.

Tabelle 5: Überblick über die verwendeten Messinstrumente

Tabelle 6: Bedeutung der Bewertungskategorien des PQS und Häufigkeit ihrer erlaubten Belegung.

Tabelle 7: Werte der Intraklassenkorrelation getrennt nach psychodynamischen und kognitivbehavioralen Behandlungssitzungen.

Tabelle 8: 10 charakteristischste Items der gesamten Stichprobe, ihre Rangordnung und Bedeutung.

Tabelle 9: 10 uncharakteristischste Items der gesamten Stichprobe, ihre Rangordnung und Bedeutung.

Tabelle 10: PQS-Items, die signifikant charakteristischer für die kognitiv-behaviorale Therapie sind.

Tabelle 11: PQS-Items, die signifikant charakteristischer für die psychodynamische Therapie sind.

Tabelle 12: Korrelationen der individuellen Q-Sorts der Patienten mit den beiden Prototypen und ihre $\mathrm{z}$-Werte.

Tabelle 13: Korrelationen (Pearson) mit den Prototypen und ihre z-Standardisierung.

Tabelle 14: Korrelationen zwischen der Orientierung am jeweiligen Prototyp mit den Outcome-Maßen.

Tabelle 15: Korrelationen zwischen „Orientierung am eigenen Prototyp“ und den OutcomeMaßen.

Tabelle 16: Die jeweils zehn charakteristischsten und uncharakteristischsten Items der verschiedenen Teilstichproben.

Tabelle 17: Ergebnisse der linearen Regressionen für alle Outcome-Maße und alle untersuchten Stichproben.

Tabelle 18: Ergebnisse der multiplen Regressionen zu den jeweils zehn charakteristischsten Items. 


\section{ii. Verzeichnis der Abbildungen}

Abbildung 1: Vereinfachtes Störungsmodell der Generalisierten Angststörung aus verhaltenstherapeutischer Sicht (nach Leichsenring et al., 2002).

Abbildung 2: Schematische Darstellung des PQS-Trainings 


\section{Zusammenfassung}

\section{Zusammenfassung}

In der vorliegenden Studie wurde der Therapieprozess von 42 Patienten untersucht. Diese Patienten stammen aus einer von der DFG geförderten Studie zur psychotherapeutischen Behandlung von Personen mit einer Generalisierten Angststörung. Durch Randomisierung wurden 19 dieser Patienten einer tiefenpsychologisch fundierten und 23 einer kognitivbehavioralen Kurzzeit-Psychotherapie zugeteilt. Für beide Psychotherapien wurde jeweils ein Manual entwickelt, nach dem die insgesamt 9 Therapeuten die Patienten zwischen 2001 und 2005 behandelten. Zur Analyse des Therapieprozesses wurden Audiomitschnitte von jeweils der 10. und 18. Sitzung mit dem Psychotherapie Prozess Q-Sort (PQS) von 3 unabhängigen Ratern beurteilt. Mit Hilfe der 100 Items des PQS werden 3 zentrale Bereiche des Therapieprozesses (emotionaler Zustand des Patienten bzw. Verhalten in der Therapie, Interaktion zwischen Patient und Therapeut bzw. in der Sitzung thematisierte Inhalte, therapeutische Interventionen und Verhaltensweisen) erfasst und auf einer neunstufigen Skala von „extrem uncharakteristisch“ bis „extrem charakteristisch“ beurteilt. Die Beobachterübereinstimmung der Rater lag bei .86. Zwischen der 10. und 18. Sitzung zeigten sich keine bedeutsamen Unterschiede hinsichtlich aller 100 Prozessvariablen. Auch zwischen der kognitiv-behavioralen und der tiefenpsychologisch fundierten Therapie zeigten sich bei 70 von 100 Prozessmerkmalen keine signifikanten Unterschiede. 33 davon beschreiben den emotionalen Zustand des Patienten bzw. sein Verhalten in der Therapie. Von den 30 Prozessmerkmalen, bei denen es zwischen beiden Therapieansätzen bedeutsame Differenzen gab, beschreiben 19 therapeutische Interventionen und Haltungen. Weiter wurde überprüft, inwieweit die beiden Therapien mit einer, gemäß Therapietheorie ideal verlaufenden Therapiesitzung übereinstimmten. Die Orientierung der tiefenpsychologisch fundiert arbeitenden Therapeuten am psychodynamischen Ideal unterschied sich nicht von der am kognitiv-behavioralen Ideal. Die kognitiv-behavioral arbeitenden Therapeuten orientierten sich signifikant mehr am kognitiv-behavioralen Ideal. In der kognitiv-behavioral behandelten Gruppe hing die Orientierung am psychodynamischen Ideal außerdem positiv mit dem Therapieoutcome zusammen. Sie führte zu einer bedeutsamen Reduktion des für Patienten mit GAS typischen Sorgenverhaltens. Die erfassten Prozessvariablen wiesen insgesamt nur wenig Vorhersagekraft für den Therapieerfolg auf. Die vorliegenden Befunde werden im Hinblick auf die Bedeutung von Therapieprozessforschung und detaillierten Kenntnissen über Wirkfaktoren und die Abläufe innerhalb einer Psychotherapie, auch unabhängig von ihrem theoretischen Hintergrund, diskutiert. 


\section{Einleitung}

\section{Einleitung}

Etwa jeder dritte Erwachsene im Alter zwischen 18 und 65 Jahren erkrankt im Laufe eines Jahres an einer psychischen Störung. Dies sind allein in Deutschland über 16 Millionen Menschen (Bundespsychotherapeutenkammer, BPTK, 2007). Allerdings nehmen nur $3 \%$ der Betroffenen eine psychotherapeutische Behandlung auf (BPTK, 2007). So berichtet die Bundespsychotherapeutenkammer (2007) von nur rund 700.000 Patienten, die innerhalb eines Jahres ambulant oder stationär psychotherapeutisch behandelt werden. Allerdings wurden Beratungsstellen, Ambulanzen und Tageskliniken hier nicht berücksichtigt. Mögliche Gründe für diese zurückhaltende Inanspruchnahme psychotherapeutischer Leistungen sind einerseits der Mangel an Informationen und Aufklärung, andererseits die Angst vor einer Stigmatisierung (BPTK, 2007).

Obwohl die Akzeptanz psychotherapeutischer Behandlungen heute noch immer nicht so hoch ist, wie es im Sinne der Patienten wünschenswert wäre (vgl. BPTK, 2007), ist sie dennoch gestiegen. Wittchen (2001) z.B. gibt an, dass 58 \% der Personen mit einer behandlungsbedürftigen psychischen Störung einer medikamentösen oder psychotherapeutischen Behandlung zugänglich wären. Diese vermehrte Inanspruchnahme psychotherapeutischer Leistungen ist sicherlich nicht zuletzt auch dadurch zu begründen, dass Forscher in den vergangenen 30 Jahren die Effektivität psychotherapeutischer Interventionen immer wieder belegen konnten (z.B. Smith \& Glass, 1977; Wittmann \& Matt, 1986; Lambert \& Ogles, 2004).

Ausgangspunkt des wachsenden Forschungsinteresses an der Frage der Wirksamkeit von Psychotherapie war eine Behauptung Eysencks (1952), nach der Psychotherapie nicht wirksamer sei als Spontanremission. Viele Psychologen ${ }^{1}$ und Psychotherapeuten fühlten sich durch diese fundamentale Kritik an ihrer Arbeit dazu aufgefordert, Eysencks Befund durch eigene empirische Forschung zu widerlegen. Erste Meta-Analysen (z.B. Smith \& Glass, 1977; Smith, Glass \& Miller, 1980) konnten dann auch die generelle Wirksamkeit von Psychotherapie zeigen.

Neben der Frage, ob Psychotherapie überhaupt wirkt, wurde und wird noch immer die Frage nach der vergleichenden Wirkung verschiedener Psychotherapieansätze gestellt und empirisch untersucht. Zur Beantwortung dieser wurden vor allem zwei weit verbreitete und in Deutschland in die Versorgung rechtlich implementierte Therapieansätze einander

\footnotetext{
${ }^{1}$ Aus Gründen der besseren Lesbarkeit wird in der vorliegenden Arbeit bei Sammelbezeichnungen (Psychologen, Therapeuten, Patienten etc.) durchgängig die männliche Form benutzt, wobei damit männliche und weibliche Personen jeweils gleichermaßen gemeint sind.
} 
gegenübergestellt: verhaltenstherapeutisch und tiefenpsychologisch fundierte Verfahren (international v.a. als psychodynamische Verfahren bezeichnet; vgl. Rüger \& Bell, 2004). Überraschenderweise zeigten einige Studien kaum Effektivitätsunterschiede zwischen diesen beiden und auch im Vergleich zu anderen Verfahren (z.B. Grawe, 1976; Smith \& Glass, 1977; Wampold, 2001). Dieser Befund wird daher auch als das „Äquivalenzparadoxon“ (vgl. Stiles, Shapiro \& Elliott, 1986) bezeichnet. Andere Arbeiten wiederum konnten einen klaren Vorteil der kognitiv-behavioralen gegenüber den psychodynamischen Verfahren ausmachen (z.B. Giles, 1983a, b; 1984; Grawe, Donati \& Bernauer, 1994). Lambert und Ogles (2004, S. 164) kommen in ihrer Überblicksarbeit zur (vergleichenden) Effektivität von Psychotherapie zu dem Schluss, dass es einerseits zwar viele Studien gibt, die das Äquivalenzparadoxon bestätigen können, dass andererseits aber kognitiv-behaviorale Methoden bei bestimmten Störungen oder unter bestimmten Bedingungen überlegen sind (z.B. bei Phobien und Panikstörungen, bei kindlicher Aggressivität, bei psychotischem Verhalten).

Aus diesen, z.T. kontroversen Befunden entwickelte sich ein neues Forschungsgebiet innerhalb der Psychotherapieforschung. Dessen zentrales Anliegen ist die Untersuchung des Therapieprozesses und die Erforschung von Wirkfaktoren, d.h. von denjenigen Faktoren, die zu Veränderungen im Patienten führen. Wirkfaktoren sind daher als Bindeglied zwischen therapeutischen Interventionen und Veränderungen im Erleben und Verhalten des Patienten zu sehen. Prozessforschung untersucht u.a., ob den Effekten von Psychotherapien unspezifische oder spezifische Faktoren zugrunde liegen. Unter unspezifischen Wirkfaktoren werden diejenigen verstanden, die in allen Therapieansätzen als wirksame Veränderungsmomente gelten dürfen wie z.B. die therapeutische Beziehung oder die Vermittlung von Hoffnung auf „Heilung“ durch die Therapie. Diese Faktoren werden als notwendige und z.T. auch als hinreichende Agenzien psychotherapeutischer Veränderung verstanden und sollen in verschiedenen Therapieansätzen im Prinzip die gleiche Wirkung entfalten. „Spezifische Wirkfaktoren“ hingegen sind diejenigen, von denen angenommen wird, dass sie nur innerhalb eines spezifischen Therapieansatzes Veränderungen im Patienten forcieren können. Solche Faktoren werden von manchen Forschern für wichtiger für die Veränderung gehalten als die unspezifischen.

Durch die Identifikation und Präzisierung gemeinsamer und spezifischer Prozessfaktoren können demnach folgende Forschungsfragen einer Beantwortung näher gebracht werden:

- Welche Aspekte einer Psychotherapie führen überhaupt zur Besserung des Patienten?

- Welche spezifischen Wirkfaktoren und damit verbunden welche spezifischen Veränderungsmechanismen werden durch eine Psychotherapie in Gang gesetzt? 


\section{Einleitung}

- Welcher Patient mit welcher Störung und welchen Ressourcen sollte mit welchem Therapieansatz behandelt werden, damit ein bestmögliches Ergebnis erzielt werden kann (differentielle Indikation)?

Durch die Beantwortung dieser Fragen kann auch auf eine Optimierung der verschiedenen Psychotherapieverfahren hingearbeitet werden, was für die psychotherapeutische Versorgungspraxis zunehmend an Relevanz gewinnt. Denn gerade im Hinblick auf in Zukunft zu erwartende weitere Kürzungen und Einschränkungen im Gesundheitssystem ist es wichtig, empirisch fundierte Aussagen zu Fragen hinsichtlich der Wirksamkeit und Effizienz von Psychotherapie und zu Aspekten eines integrativen Therapiemodells treffen zu können (vgl. Grawe, 1995). Dazu ist es notwendig, den Therapieprozess auch über die Grenzen verschiedener Therapieschulen und -orientierungen hinweg zu untersuchen (,desegregation process research“ vgl. Shoham-Salomon, 1991; vgl. a. Wolfe \& Goldfried, 1988). Erst dann wird es möglich sein, ein integratives, therapieschulenübergreifendes Behandlungsmodell zu entwickeln. Ein solches erfordert aber immer auch eine Entscheidung darüber, was überhaupt zu integrieren ist (vgl. Goldfried, 1991). Dazu wiederum ist eine intensive Analyse der einzelnen Prozesskomponenten, so wie sie in der vorliegenden Arbeit durchgeführt werden soll, erforderlich.

Ziel der vorliegenden Arbeit ist es, einen kognitiv-behavioralen mit einem tiefenpsychologisch fundierten Behandlungsprozess von Patienten mit einem gemeinsamen Störungsbild miteinander zu vergleichen, um neue Erkenntnisse über gemeinsame und spezifische Prozessmerkmale innerhalb dieser beiden Therapiekonzepte gewinnen zu können. Auch sollen die aktiv wirksamen Elemente des jeweiligen Therapieansatzes identifiziert werden. Langfristiges Ziel kann dann sein, diejenigen Komponenten des Therapieprozesses, die sich ggf. auch unabhängig vom Therapieansatz als effektiv erwiesen haben, in ein gemeinsames Behandlungsmodell zu integrieren.

Die Wahl des Vergleichs dieser beiden Therapieansätze ist den PsychotherapieRichtlinien zu schulden. Psychotherapeuten dürfen ihre Leistungen nur dann über die Gesetzlichen Krankenkassen abrechnen, wenn sie von den Kassenärztlichen Vereinigungen zugelassen sind, eine Approbation haben und wenn sie die sog. Richtlinienverfahren anwenden (BPTK, 2007). Zu diesen Richtlinienverfahren gehören derzeit die analytische und die tiefenpsychologisch fundierte Psychotherapie sowie die Verhaltenstherapie.

Gerade weil sich diese Verfahren hinsichtlich ihrer theoretischen Konzeption stark unterscheiden, ist ein empirischer Vergleich besonders wichtig (vgl. Kazdin, 1986). Denn große konzeptuelle Unterschiede implizieren nicht unbedingt Unterschiede auf der Ebene des 
tatsächlichen therapeutischen Handelns („Übereinstimmung zwischen klinischer Theorie und klinischer Praxis ist also nicht die Regel.“, Davies-Osterkamp, Heigl-Evers, BosseSteuernagel \& Alberti, 1987, S. 24). So konnten Ambühl et al. (1995) zeigen, dass sich die therapeutische Orientierung mit zunehmender klinischer Erfahrung verändert. Auch bringen Psychotherapeuten häufig zwei oder mehr unterschiedliche theoretische Ansätze in ihre praktische Arbeit mit dem Patienten ein (vgl. a. Lambert \& Ogles, 2004; Skovholt \& Roennestad, 1995). Daher erscheint es sowohl aus einer Forscher- als auch aus einer praxisnahen Perspektive indiziert, die konzeptuellen Unterschiede zwischen psychodynamischen und kognitiv-behavioralen Therapien hinsichtlich ihrer tatsächlichen praktischen Umsetzung in konkretes therapeutisches Handeln empirisch zu untersuchen (vgl. Blagys \& Hilsenroth, 2000). So sind Aussagen über gemeinsame und/oder spezifische Wirkfaktoren möglich.

Während der Vergleich des Therapieoutcomes verschiedener Therapieansätze schon länger zur gängigen Forschungspraxis gehört, ist erst in den vergangenen beiden Jahrzehnten ersichtlich geworden, dass die alleinige Betrachtung des Therapieerfolges zur Bestimmung der wirksamen Therapieelemente und für einen fairen Vergleich unterschiedlicher Therapieansätze nicht ausreicht. Die genaue Erforschung der gemeinsamen und spezifischen Prozessfaktoren von tiefenpsychologisch fundierten und kognitiv-behavioralen Therapien erscheint daher besonders relevant (vgl. Leibing, Rabung \& Leichsenring, 2005).

Für einen validen Vergleich von Therapien ist es notwendig, dass die untersuchten Therapien manualisiert (wegen der externen Validität, vgl. Luborsky \& DeRubeis, 1984; Kendall, Holmbeck \& Verduin, 2004) in einem naturalistischen Setting durchgeführt werden und, um die Vergleichbarkeit zu gewährleisten, spezifische Störungsbilder fokussieren.

Die Datenbasis der vorliegenden Studie bilden daher die Befunde einer Psychotherapiestudie, die diese Auflagen erfüllt. In dieser von der Deutschen Forschungsgemeinschaft geförderten Studie ${ }^{2}$ zur Behandlung der Generalisierten Angststörung (GAS) von der Universität Göttingen wurden die Patienten mit einer manualisierten Psychotherapie von niedergelassenen Psychotherapeuten behandelt.

Die GAS ist eine sehr häufige Angststörung, die meist chronisch verläuft und mit schweren Beeinträchtigungen verbunden ist (Leibing, Winkelbach \& Leichsenring, 2003). Bis vor wenigen Jahren wurde ihr, trotz ihrer hohen Prävalenz, wahrscheinlich aufgrund diagnostischer Schwierigkeiten, die sich durch ungenaue Diagnosekriterien (zumindest bis

\footnotetext{
${ }^{2}$ Deutsche Forschungsgemeinschaft, DFG, Fördernummer LEI250/1-1
} 


\section{Einleitung}

zum Erscheinen des DSM-IIIR) ergaben und auch wegen der zunächst wenig dramatisch erscheinenden Symptomatik, nur wenig Forschungsinteresse entgegengebracht (vgl. Hoyer, Beesdo, Becker \& Wittchen, 2003). Auch wurde sie in der Praxis nur selten diagnostiziert (vgl. Leibing et al., 2003).

Im Rahmen der hier zugrunde liegenden GAS-Studie wurden zunächst zwei Behandlungsmanuale entwickelt, die auf einem psychodynamischen bzw. kognitivbehavioralen Behandlungsansatz basierten. Die Patienten wurden jeweils einem der beiden Behandlungsarme randomisiert zugeteilt und behandelt. Der erste Teil der Studie beinhaltete eine komparative Outcome-Untersuchung mit dem Ziel, neue Erkenntnisse über die vergleichende Effektivität dieser beiden Therapieprogramme gewinnen zu können. Beide Therapien erwiesen sich als hochwirksam. Auch zeigten sich in Bezug auf das Hauptoutcomemaß keine signifikanten Differenzen zwischen der psychodynamischen und der kognitiv-behavioralen Therapie (Winkelbach, 2007).

Mit der vorliegenden Untersuchung soll nun in einem zweiten Untersuchungsschritt der Psychotherapieprozess dieser beiden Therapieansätze miteinander verglichen werden. Dies soll mit Hilfe eines Q-Sort-Verfahrens geschehen, wodurch Aussagen über Prozessfaktoren möglich werden. Auch soll überprüft werden, ob und wenn ja, welche dieser Prozessfaktoren mit dem Outcome der Therapie zusammenhängen und ob diese Wirkfaktoren als spezifische oder unspezifische Wirkfaktoren zu interpretieren sind. Weiterhin wird untersucht, wie typisch die beiden hier implementierten Therapien für die jeweils zugehörigen Therapietheorien sind und ob die laut Therapietheorie für Veränderungen im Patienten verantwortlichen Therapieprozessmerkmale auch in der Empirie die erwünschten Veränderungen erzielen konnten. 


\section{Theoretischer und empirischer Hintergrund}

\section{Theoretische und empirische Fundierung der kognitiv-behavioralen und der psychodynamischen Psychotherapie}

In der vorliegenden Arbeit wird der Therapieprozess einer kognitiv-behavioralen und einer tiefenpsychologisch fundierten Psychotherapie miteinander verglichen. Psychotherapie (griechisch: psychotherapía = Seelendienst/ Heilung der Seele) wird dabei als „... the informed and planful application of techniques derived from established psychological principles, by persons qualified through training and experience to understand these principles and to apply these techniques with the intention of assisting individuals to modify such personal characteristics as feelings, values, attitudes, and behaviors which are judged by the therapist to be maladaptive or maladjustive." (vgl. Smith \& Glass, 1977) verstanden.

\subsection{Kognitiv -behaviorale Ansätze}

\subsubsection{Grundprinzipien und historische Entwicklung}

Verhaltenstherapie wird heute nicht als homogenes Verfahren, sondern als eine heterogene Gruppe von Therapieansätzen mit spezifischen Modifikationszielen verstanden (vgl. Kröner-Herwig, 2004). Die gemeinsamen Momente liegen in einem lerntheoretischen Verständnis der Genese und Therapie von Störungen (vgl. Kriz, 1994) sowie in einer empirischen Orientierung (vgl. Margraf, 2000). Die Verwendung des Begriffes „Verhaltenstherapie“ ist durch die klinische Anwendung der durch psychologische Forschung etablierten Prinzipien der Lerntheorien zu erklären (vgl. Margraf, 2000). Therapeutische Interventionen, wie sie im Rahmen einer kognitiv-behavioralen Psychotherapie zur Anwendung kommen, basieren auf wissenschaftlichen Modellen menschlichen Verhaltens (z.B. auf Lernmodellen) und von Kognitionen und Emotionen (Dobson, 2000). Hier wird davon ausgegangen, dass psychische Störungen erworbene bzw. ungünstige Verhaltensweisen und Kognitionen darstellen, die sich qualitativ nicht von „,normalem“ Verhalten unterscheiden. Ziel der Interventionen ist eine Veränderung des Verhaltens und der intern ablaufenden Prozesse, was zu einer verbesserten sozialen Anpassung, zu mehr Selbstkontrolle und Problemlösekompetenz sowie zu einer Minderung des Leidensdrucks und der subjektiv empfundenen Beeinträchtigung des Patienten beitragen soll (vgl. Kröner-Herwig, 2004). Im Rahmen des generellen Interventionsprinzips der Verhaltenstherapie lernt und übt der Patient neues, für ihn zunächst noch schwieriges Verhalten unter Realbedingungen, wobei er sich 
seinen Zielen geplant approximativ nähert. Die unterschiedlichen verhaltenstherapeutischen Ansätze basieren auf einigen gemeinsamen Grundprinzipien (vgl. Margraf, 2000). Im Rahmen einer Problem- und Verhaltensanalyse werden die genetischen, sozialen, somatischen, kognitiven, emotionalen und behavioralen prädisponierenden, auslösenden und aufrechterhaltenden Bedingungen der Störung erarbeitet (,,biopsychosoziales Modell“‘). Gemeinsam mit dem Patienten werden daraus spezifische und konkrete (Therapie-)Ziele für die Gegenwart und die Zukunft abgeleitet. Der Fokus der Arbeit in der Therapie liegt im Hier und Jetzt (vgl. Leichsenring, Hiller, Weissberg \& Leibing, 2006). Durch aktive Beteiligung wird der Patient zum Experten seiner eigenen Störung, was die Generalisierung des in der Therapie erlernten Verhaltens auf den Alltag ermöglichen soll (z.B. über Hausaufgaben).

Verhaltenstherapeutische Techniken für die klinische Praxis entwickelten sich in den 50er und 60er Jahren (Reinecker, 2005). Erkenntnisse zum instrumentellen und operanten Lernen und zur Klassischen Konditionierung wurden in technologische Regeln überführt und auf das therapeutische Vorgehen adaptiert und angewendet (vgl. Kröner-Herwig, 2004). Das Interventionswissen ist demnach keine Ableitung aus Lerntheorien, sondern vielmehr deren heuristische Nutzanwendung (Kröner-Herwig, 2004).

Eines der ersten störungsspezifischen Interventionsprogramme der Verhaltenstherapie ist die „Systematische Desensibilisierung“(Wolpe, 1958). Sie dient dem Angstabbau. Wolpe (1958) nahm als ursächlichen Wirkfaktor die „reziproke Hemmung“ an, was heute jedoch als widerlegt gelten muss (vgl. Maercker, 2003). Darunter wird verstanden, dass zwei sich widersprechende Reaktionen (wie Angst und Entspannung) nicht gemeinsam auftreten können. Deshalb lernt der Patient, in Angstsituationen ein Entspannungsverfahren wie die Progressive Muskelrelaxation (Jacobson, 1938) einzusetzen.

Verhaltenstherapeutische Ansätze entwickelten sich durch den Einbezug des „DreiEbenen-Ansatzes“ (z.B. Lang, 1965, 1993) weiter. Dieser geht davon aus, dass bei der Betrachtung psychischer Störungen sowohl verhaltensmäßige und physiologische als auch affektive bzw. kognitive Reaktionen berücksichtigt werden müssen. Eine psychische Störung äußert sich immer auf allen drei Ebenen, wobei diese aber weder in ihrem zeitlichen Verlauf der Entwicklung noch in ihrem Modifikationsverlauf synchron verlaufen müssen (Hodgson \& Rachman, 1974). Statt von einem Drei- wird oft von einem Vier-Ebenen-Ansatz gesprochen, wenn die kognitive und die affektive Ebene getrennt voneinander betrachtet werden.

Kognitive Therapietheorien etablierten sich zunächst unabhängig von den klassisch behavioralen. Erst später, als die Bedeutung der Berücksichtigung innerer Prozesse immer 
deutlicher wurde (,kognitive Wende“), gab es erste Bemühungen zu ihrer Integration (,kognitiv-behaviorale Therapie“). Banduras (1969) Konzept des „Modellernens“ bspw. integriert lerntheoretische und kognitive Modellannahmen z.B. durch den Einbezug kognitiver Repräsentationen. Komplexes soziales und funktionales Verhalten soll demnach durch Beobachtung und Imitation des Verhaltens anderer Menschen erlernt werden.

Eine für das heutige Verständnis von „kognitiv-behavioraler Therapie“ besonders wichtige Therapietheorie ist Becks „Kognitive Therapie“ (Beck, 1967). Dieser Therapieansatz wurde zunächst nur für Menschen mit Depressionen entwickelt, wurde aber später auch auf Menschen mit Angst- oder Zwangsstörungen adaptiert. Depressive Menschen zeigen demnach typische „Denkmuster“ (,,automatische Gedanken“) in Form einer negativen Sichtweise der Umwelt, der Zukunft und sich selbst („kognitive Triade“). Solche automatischen dysfunktionalen Gedanken (und auch problematisches Verhalten) sollen im Rahmen der Therapie identifiziert und mit Hilfe kognitiver Methoden wie Disputation, Realitätsüberprüfung im Verhaltensexperiment, Reattribuierung oder kognitiver Umstrukturierung, d.h. Infragestellung und Modifikation negativer kognitiver Schemata, verändert werden.

Für die Weiterentwicklung der Verhaltenstherapie wichtige, störungsübergreifend geltende Überlegungen stammen auch aus dem „Selbstmanagementansatz“ von Kanfer (z.B. Kanfer \& Philips, 1975), der als eine Art „Metamodell des Therapieprozesses“ (vgl. Reinecker, 2005, S. 290) verstanden werden kann. Danach können Menschen ihr Verhalten selbst steuern, in dem sie sich selbst beobachten, ihr Verhalten anhand von Standards bewerten und sich je nach Ergebnis dieser Bewertung positiv oder negativ verstärken oder bestrafen (,Selbstkontrolle“ $\rightarrow$,Selbstbeobachtung“ $\rightarrow$,Selbstbewertung“ $\rightarrow$ „Selbstverstärkung“). Kanfer (1977) bezeichnet diesen Prozess als „Selbstregulation“.

In den 80er Jahren etablierte sich als weiterer „Stützpfeiler“ (vgl. Kröner-Herwig, 2004) der Verhaltenstherapie die „Verhaltensmedizin“. Darunter wird die Anwendung psychologischer Modifikationsverfahren im Bereich somatischer Krankheiten verstanden (vgl. Kröner-Herwig, 2004).

Mittlerweile gibt es viele verhaltenstherapeutische Programme zur Behandlung spezifischer Störungen, die in Form eines Manuals explizit formulierte und detaillierte Therapieanweisungen und Materialien zur Verfügung stellen (wie z.B. das in dieser Studie verwendete kognitiv-behaviorale Behandlungsmanual zur Therapie der GAS nach Leibing et al., 2003; vgl. 4.3.2 in diesem Kapitel). Neben diesen störungsspezifischen 
Theoretischer und empirischer Hintergrund

Theoretische und empirische Fundierung der kognitiv-behavioralen und der psychodynamischen Psychotherapie

Therapieprogrammen werden auch störungsübergreifende Maßnahmen (z.B.

Problemlösetrainings) sowie allgemein notwendige Basisfertigkeiten (Beziehungsgestaltung, Motivationsarbeit, Gesprächsführung) eingesetzt (vgl. Margraf, 2000, S. 8).

Kognitiv-behaviorale Interventionen sind demnach strukturierte Maßnahmen. Dies wird durch die zunehmende Verwendung von Therapiemanualen (s.o.) zusätzlich unterstützt. Der Therapeut lenkt und strukturiert die einzelnen Sitzungen und wirkt deshalb auch aktiv auf den therapeutischen Prozess ein. Die therapeutische Beziehung wird als wichtige, wenngleich auch nicht hinreichende Bedingung der durch die Psychotherapie erreichten Veränderung betrachtet. $\mathrm{Zu}$ ihrer Gestaltung werden Methoden wie Akzeptanz und Empathie aus anderen therapeutischen Richtungen (z.B. der Gesprächspsychotherapie nach Rogers) entlehnt (vgl. Kröner-Herwig, 2004).

\subsubsection{Stand der Outcome-Forschung}

Zur kognitiv-behavioralen Therapie gibt es mittlerweile eine nahezu unüberschaubare Menge an randomisierten und kontrollierten klinischen Studien. Die Effektivität dieses Verfahrens ist bei folgenden Störungen belegt (vgl. Leichsenring et al., 2006, zur genaueren Beschreibung dieser Untersuchung vgl. 2.2 in diesem Kapitel): bei depressiver Störung, bei Panikstörung mit und ohne Agoraphobie, bei Sozial- und spezifischer Phobie, bei Bulimia Nervosa, bei Posttraumatischer Belastungsstörung, bei Schizophrenie hinsichtlich der Zunahme an kompetentem sozialen Verhalten und sozialen Fähigkeiten, bei Generalisierter Angststörung, bei somatoformer und Schmerzstörung, bei Hypochondrie, bei Alkoholmissbrauch und bei bipolaren Störungen.

\subsubsection{Wirkfaktoren der kognitiv-behavioralen Therapie}

Bisher gibt es keine homogene Wirktheorie. Es wird jedoch davon ausgegangen, dass psychotherapeutischen Veränderungen kein einzelner Wirkfaktor zugrunde liegt, sondern dass mehrere Faktoren gleichzeitig an einer solchen Veränderung beteiligt sind. Diese Faktoren wirken nicht linear, sondern entfalten erst in einem komplexen, prozesshaften und dynamischen Modell ihre volle Wirkung. So ist in diesem Rahmen z.B. vorstellbar, dass eine bestimmte therapeutische Intervention zu einer Veränderung auf der behavioralen, gleichzeitig aber auch auf der emotionalen, kognitiven und physiologischen Ebene führt. Veränderte Reaktionen der Umwelt könnten die Folge einer solchen Veränderung im Patienten sein. 
Laireiter (2000) unterscheidet zwischen Wirkfaktoren erster und zweiter Ordnung und zwischen spezifischen und unspezifischen Bedingungen. Wirkfaktoren erster Ordnung kennzeichnen die notwendigen und hinreichenden Bedingungen in der Therapie, über die Veränderungen erzielt werden können (spezifische, methoden- und technikbezogene Wirkfaktoren). Behavioristische Lerntheorien (klassische und operante Konditionierung), Modellernen, kognitive Theorien sowie das Selbstregulationsmodell (Kanfer \& Phillips, 1975) gehören nach dieser Auffassung dazu. Weiterhin sollen auch „Einsicht““, „Bewusstwerdung“, „Klärung“ und „Entwicklung neuer Sichtweisen“ relevante spezifische Wirkfaktoren im kognitiv-behavioralen Therapieprozess darstellen. Wirkfaktoren zweiter Ordnung sind solche, die zwar notwendig, aber nicht hinreichend für therapeutische Veränderungen sind. Dies sind nach Laireiter (2000) Merkmale des Therapeuten, des therapeutischen Prozesses und der Therapeut-Patient-Beziehung. Da diese Faktoren aber in allen Therapieansätzen wirken, werden sie als unspezifische Wirkfaktoren verstanden (vgl. 3.5 in diesem Kapitel).

Insgesamt sind die Wirkmechanismen kognitiv-behavioraler Therapien weniger gut untersucht als ihre Wirksamkeit. Somit ist der Wissenstand darüber, welche Interventionen zu welchen Veränderungen mit welchen Konsequenzen führen, noch nicht ausreichend (vgl. Kröner-Herwig, 2004).

\subsection{Psychodynamische Ansätze}

\subsubsection{Historische Entwicklung und Grundprinzipien}

Mertens (2005, S. 197) weist darauf hin, dass es die Psychoanalyse oder die psychoanalytische Therapie nicht mehr gibt. Stattdessen existiert heute eine Vielzahl von unterschiedlichen Auffassungen darüber, was wesentliche Bestandteile der psychoanalytischen Theorie und Therapie sind. Diese lassen sich jedoch alle in irgendeiner Form aus der klassischen Psychoanalyse nach S. Freud (z.B. 1913, 1914, 1923, 1937) ableiten (vgl. Hoffmann, 2000). Freud selbst veränderte seine Auffassung über „die Psychoanalyse“ immer wieder. Zunächst entwickelte er die sog. „Traumatheorie“. Danach soll ein z.B. durch Inzest ausgelöstes Trauma über eine Blockierung der Affekte zu einer Verdrängung und in deren Folge zu einer psychischen Störung führen. Im Rahmen der „Triebtheorie“ hingegen sollen Verhalten und Erleben, aber eben auch psychische Symptome durch Konflikte zwischen verschiedenen Trieben entstehen. Die Triebtheorie wiederum wurde vom „Strukturmodell“ abgelöst. Danach entstehen Störungen durch Konflikte zwischen den 
Triebwünschen des Es und den moralischen Standards des Über-Ichs. Das Ich arbeitet nach dem Realitätsprinzip und versucht zwischen Es und Über-Ich zu vermitteln.

Neben seinen Theorien zur Entstehung psychischer Störungen entwickelte Freud ein Konzept, wie eine idealtypische psychoanalytische Behandlung aussehen sollte (z.B. Freud, 1912). Danach ist die Grundregel jeder Therapie die „freie Assoziation“. Dies bedeutet, dass der Patient in der Therapie all das sagt, was ihm in den Sinn kommt, auch wenn es ihm unsinnig, peinlich oder unwichtig erscheint (vgl. Streeck, 2000). Um ihm dies zu erleichtern und die Regression (s.u.) zu fördern, liegt der Patient in der klassischen Psychoanalyse auf einer Couch. Der Therapeut sitzt so hinter ihm, dass er ihn nicht sehen kann und daher vom ihm (weitgehend) unbeeinflusst bleibt. Der Therapeut begegnet dem Patienten gleichschwebend aufmerksam, indem er das vom Patienten Gesagte weder wertet oder gewichtet noch logisch ordnet. Weiterhin wird vom Analytiker Neutralität gefordert. Dies bedeutet, dass er sich als Person mit seinen eigenen Vorstellungen und persönlichen Eigenschaften nicht zeigt. Hierdurch erleichtert er es dem Patienten, Phantasien zuzulassen und zu verbalisieren. Mit der therapeutischen Beziehung erlebt der Patient eine ungewöhnliche Beziehung, in der er verstanden und gehalten wird und die es ihm ermöglicht, bislang verpönte, angstbesetzte Affekte und Impulse wieder zu erleben. Die Beziehung zwischen Patient und Therapeut ist daher ,,intensiv emotional“ (Streeck, 2005, S. 249) und durch das Konzept der Übertragung geprägt. In der Übertragung werden quasi „,geronnene“ verinnerlichte Konflikte wieder zu interpersonellen Konflikten und können in der Beziehung zum Analytiker durchgearbeitet und gelöst werden. „Der Therapeut spürt sozusagen am eigenen Leib, wie man sich als Beziehungspartner des Patienten fühlt.“ (Faller, 2004, S. 135). Aber auch aufgrund gegenwärtiger Wünsche, Erwartungen und Charakterzüge kann es zur Übertragung kommen. Die Gefühle, Vorstellungen und Phantasien, die durch Übertragung beim Therapeuten ausgelöst werden, werden als Gegenübertragung bezeichnet. Der Therapeut soll diese als therapeutisches Vehikel verstehen, zu diagnostischen Zwecken nutzen und keinesfalls abreagieren. Seine Aufgabe ist es daher, Übertragung und Gegenübertragung zu erkennen, zu deuten (Verbalisierung des für den Therapeuten latenten Sinngehalts) und mit dem Patienten durchzuarbeiten. So ermöglicht er ihm neue Erfahrungen in einem gesicherten, angstfreien therapeutischen Raum, die dieser in sein neues Selbstverständnis und in seine außertherapeutischen Beziehungen übernehmen kann. Der Patient kann z.B. die Erfahrung machen, dass der Analytiker ihn nicht ablehnt, wenn er aggressiv wird (korrigierende emotionale Erfahrung). Da Deutung und Durcharbeitung von 
Material innerhalb und außerhalb der Übertragung jedoch abgewehrte unbewusste Konflikte aufdecken oder das Eingestehen beschämender oder Selbstwert verunsichernder Erfahrungen erfordern kann, reagieren Patienten mit (bewusstem oder unbewusstem) Widerstand (z.B. Zuspätkommen des Patienten, small talk, gähnen etc.). Der Widerstand dient dem Patienten zur Regulierung des Tempos, in dem unbewusste Konflikte dem Bewusstsein zugänglich gemacht werden. Er begleitet die gesamte Analyse und hat eine wichtige Funktion. Der Therapeut deutet diesen Widerstand und arbeitet ihn ebenfalls mit dem Patienten durch.

In Anlehnung an Pine (1990) können innerhalb der psychoanalytischen Denktradition vier psychoanalytische „Psychologien“ unterschieden werden. Diese formulieren jeweils etwas andere Annahmen darüber, welche Dynamik seelischen Prozessen zugrunde und wo der Schwerpunkt der Arbeitsweise der menschlichen Psyche liegt. Die daraus abgeleiteten Behandlungsverfahren sind deshalb auch entsprechend vielfältig.

1. Die Triebpsychologie geht davon aus, dass jeder Mensch Wünsche und Bedürfnisse (Triebe) hat, die eine biologische Grundlage haben und sich unter dem Einfluss von Umweltfaktoren entwickeln. Diese werden in Wünschen, Affekten, Handlungen und bewussten und unbewussten Phantasien verkörpert. Konflikte entstehen dadurch, dass einige dieser Wünsche als unannehmbar und gefährlich erlebt werden oder tatsächlich nicht befriedigt werden können oder konnten und so zu unvermeidbaren Frustrationen führen (z.B. Geburt eines Geschwisters). Gegen unannehmbare Wünsche werden Abwehrmechanismen eingesetzt wie z.B. Verdrängung und Projektion. Hierdurch werden die angstbesetzten Wünsche sowie die damit verbundene Angst oder Schuldgefühle unbewusst abgewehrt; die Konfliktspannung nimmt ab (primärer Krankheitsgewinn). Durch spezifische Versuchungs und Versagungssituationen können die abgewehrten Impulse jedoch später wieder reaktiviert werden. Das Symptom stellt hier einen Kompromiss zwischen Impuls und Abwehr dar und enthält damit beide Komponenten. Es handelt sich um verinnerlichte Konflikte, nicht um Konflikte zwischen dem Patienten und Personen der Außenwelt. Diese Form der Symptomentstehung wird als „Konfliktpathologie“ bezeichnet. Ziel einer Therapie, die Konfliktpathologien fokussiert, ist es, desintegrierte affektive Erfahrungen bzw. Konflikte in der Therapie wieder zu erleben, dem Patienten so bewusst zu machen und dadurch in die Gesamtstruktur der Person zu integrieren. Dies soll über regressive Prozesse geschehen, in deren Rahmen der Patient unbewusste Konflikte auf den Analytiker überträgt und so neue korrigierende emotionale Erfahrungen sammeln kann (s.o.). 
Theoretischer und empirischer Hintergrund

Theoretische und empirische Fundierung der kognitiv-behavioralen und der psychodynamischen Psychotherapie

Diese „Konfliktpathologie“ steht im Gegensatz zur „Strukturpathologie“, bei der IchFunktionen nicht ausreichend entwickelt sind (z.B. Impulskontrolle bei Borderline-Patienten). Diese Störungen werden von der Ich-Psychologie, der Selbstpsychologie und der Objektbeziehungstheorie beschrieben und mit Hilfe einer psychoanalytisch-interaktionellen Psychotherapie (s.u.) behandelt.

2. In der Ich-Psychologie werden unter Ich-Funktionen psychosoziale Kompetenzen wie Frustrationstoleranz, Impulskontrolle oder Realitätsprüfung verstanden (Bellak, Hurvich \& Gediman, 1973). Diese Fähigkeiten werden im Rahmen zwischenmenschlicher Beziehungen im Lauf der kindlichen Entwicklung ausgebildet. Allerdings gelingt dies nicht allen Menschen gleichermaßen gut.

3. Bei der Psychologie der Objektbeziehungen stehen interpersonelle zwischenmenschliche Beziehungen im Fokus der Aufmerksamkeit. Menschliche Entwicklung findet in einer Beziehungsmatrix statt, die bestimmend für spätere Beziehungen, aber auch für die Entwicklung der Selbstorganisation ist (vgl. Mertens, 2000). Zwischenmenschliche Erfahrungen werden in Form von Selbst- und Objektrepräsentanzen verinnerlicht (z.B. Kernberg, 1988). Mit ihnen können pathologische Überzeugungen einhergehen, wie z.B. „Wenn ich aggressiv bin (Selbstrepräsentanz), mögen die anderen mich nicht mehr (Objektrepräsentanz)“.

4. Bei der Psychologie des „Selbst-Erlebens“ (Pine, 1990) geht es in erster Linie um das Bild, das eine Person von sich selbst hinsichtlich ihrer Grenzen, Kontinuität und Wertschätzung und in Bezug auf ihre Reaktion auf Schwankungen dieses subjektiven Zustands hat (vgl. Kohut, 1973; Kernberg, 1988). Oftmals konnte das Bedürfnis nach Selbstkohärenz von wichtigen Bezugspersonen nicht befriedigt werden, sodass diese Person als Konsequenz nach übermäßiger Anerkennung und/oder nach Personen, die sie idealisieren kann, sucht.

Ziel der ,psychoanalytisch-interaktionellen Psychotherapie“ ist es, diese grundlegenden strukturellen Störungen zu überwinden, indem seelische und interpersonelle Fähigkeiten, die der Realität angemessener sind, zur Nachentwicklung gebracht und gestärkt werden (vgl. Streeck, 2000, S. 246). Mit deren Hilfe können dann wiederum z.B. reifere Objektbeziehungen und Ich-Funktionen entwickelt werden. Da diese aber nicht aufgrund unbewusster Konflikte beeinträchtigt sind, ist es nicht möglich, deren Entwicklung durch Regression zu fördern. In der Regression würde der Patient sich vorübergehend auf einem in der Entwicklung zurückliegenden seelischen Entwicklungsniveau befinden (vgl. Streeck, 
Theoretischer und empirischer Hintergrund

Theoretische und empirische Fundierung der kognitiv-behavioralen und der psychodynamischen Psychotherapie

2000). In der progressionsorientierten interaktionellen Psychotherapie, die zu den tiefenpsychologisch fundierten Verfahren zählt, werden stattdessen Bedingungen geschaffen, die dem Patienten diese Nachentwicklung und das Verinnerlichen neuer Beziehungserfahrungen ermöglichen. Die Aufgabe des Therapeuten besteht deshalb stärker darin, dem Patienten gegenüber die eigenen Gefühle transparent zu machen, die sich bei ihm in Reaktion auf den Patienten einstellen. Er achtet des Weiteren besonders auf die Interaktion mit dem Patienten und weniger auf den verborgenen Sinn in dessen Äußerungen (vgl.

Streeck, 2000).

\subsubsection{Unterscheidung von tiefenpsychologisch fundierter und analytischer Psychotherapie}

In Deutschland werden tiefenpsychologisch fundierte von analytischen Psychotherapien unterschieden. Beide sollen bei denjenigen Störungen indiziert sein, bei denen (unbewusste) intrapsychische und/oder interpersonelle Konflikte in einem plausiblen Zusammenhang mit körperlichen und psychischen Symptomen stehen (vgl. a. Richter, 1998). Gemeinsam ist diesen Ansätzen, dass sie die unbewusste Psychodynamik neurotischer Störungen (mit psychischer und/oder somatischer Symptomatik) zum Gegenstand der Behandlung machen (vgl. Richter, 1998). Das Ausmaß der Regressionstiefe und der Umgang mit dem Unbewussten, der Gegenstandsbereich der Therapie, das Setting und die Dauer, in dem die Behandlung stattfindet, sowie ihre Frequenz sind wesentliche Kriterien, nach denen diese beiden Therapiekonzepte voneinander unterschieden werden können.

In der analytischen Psychotherapie werden mit Hilfe von Übertragungs-, Gegenübertragungs- und Widerstandsanalysen neurotische Symptomatiken, neurotische Konflikte sowie zugrunde liegende neurotische Strukturen (d.h. intrapsychische Konflikte) behandelt, indem regressive Prozesse gezielt gefördert werden. Dazu werden in der Regel zwei- bis dreimal wöchentlich insgesamt ca. 160-240 Behandlungsstunden (also etwa 2-3 Jahre) benötigt, in denen der Patient im klassischen Couch-Sessel-Setting behandelt wird. Der Therapeut hält die Abstinenz ein. Ziel ist eine strukturelle Veränderung bzw. eine Umstrukturierung der Persönlichkeit sowie des Verhältnisses der drei Instanzen Es, Ich und Über-Ich, was dem Patienten ein tieferes Verständnis seiner selbst sowie eine größeres Ausmaß an innerer und äußerer Beweglichkeit und Freiheit ermöglichen soll.

Der Begriff der „tiefenpsychologisch fundierten Therapie“ wurde erstmals 1967 von Winkler in die Debatte über Richtlinien-Psychotherapie eingeführt (vgl. Reimer \& Rüger, 2003). International wird dafür v.a. der Begriff ,,psychodynamische (Psycho-)Therapie“ 
Theoretischer und empirischer Hintergrund

Theoretische und empirische Fundierung der kognitiv-behavioralen und der psychodynamischen Psychotherapie

gebraucht $^{3}$ (vgl. Rüger \& Reimer, 2003). Psychodynamische Therapien lassen sich auf einem Kontinuum von expressiv bis supportiv einordnen (vgl. Leichsenring et al., 2006). Expressive Interventionen sollen dem Patienten dabei helfen, Einsicht in seine wiederkehrenden Konflikte, die seine Probleme aufrechterhalten, zu erlangen. Supportive Interventionen hingegen sollen stärker die „Ich-Funktionen“ des Patienten stärken (vgl. Leichsenring et al., 2006). Anders als die analytische Psychotherapie beziehen psychodynamische Psychotherapien auch andere konzeptuelle Grundlagen wie z.B. lerntheoretische oder transaktionale mit ein (vgl. Rüger \& Reimer, 2003). Die Behandlungen dauern zwischen 2580 Sitzungen (ca. 1/2 bis 2 Jahre, 1-2mal wöchentlich). Patient und Therapeut sitzen sich gegenüber. Es werden aktuell wirksame neurotische Konflikte sowie deren gegenwärtige pathologische Kompromissbildungen in das Zentrum der Aufmerksamkeit gestellt. Aus diesem Fokus werden Ziele und Teilziele der Therapie abgeleitet und in den einzelnen Sitzungen gezielt, d.h. konfliktzentriert, bearbeitet (vgl. Richter, 1998). Deswegen spielen Deutungen gegenüber themenbestimmenden, fokussierenden, leitenden oder klärenden Fragen eine geringere Rolle und die Regression wird weniger intensiv gefördert. Der Therapeut ist relativ aktiv (vgl. Leibing et al., 2005, S. 372), auch ist er supportiver als in der analytischen Psychotherapie. Übertragung, Gegenübertragung und Widerstand werden zwar beachtet, jedoch ausdrücklich weniger stark fokussiert. Die definierten und begrenzten Konflikte werden in ihrem Entstehungszusammenhang herausgearbeitet und gedeutet. Der Fokus der Erfahrung liegt im „Hier und Jetzt“ (vgl. Leibing et al., 2005, S. 372). Eine wichtige Voraussetzung für das Gelingen der Therapie ist eine tragfähige Therapeut-PatientBeziehung, die der Therapeut ebenfalls aktiv fördert (vgl. Leibing et al., 2005, S. 372). Ziel der Therapie ist weniger eine Umstrukturierung der Persönlichkeit als vielmehr eine stabilere und flexiblere Anpassung des Patienten an äußere Lebensumstände (Streeck, 2000). Eine einheitliche Theorie zur tiefenpsychologisch fundierten Psychotherapie existiert allerdings bislang noch nicht ${ }^{4}$.

\footnotetext{
${ }^{3}$ Nach Hoffmann (2000) werden mit dem Begriff der ,psychodynamischen Therapie“ diejenigen Psychotherapieformen bezeichnet, die sich in irgendeiner Form auf die Psychoanalyse beziehen oder von ihr abgeleitetet sind. Begriffe wie „tiefenpsychologisch fundierte Psychotherapie“ und „,analytische Psychotherapie“ sind international zwar nicht gebräuchlich, werden im deutschsprachigen Raum jedoch häufig benutzt. In der hier vorliegenden Arbeit werden die Begriffe „tiefenpsychologisch fundierte Therapie“ und „psychodynamische (Psycho)-Therapie“ daher synonym verwendet.

${ }^{4}$ Eine ausführlichere Darstellung der Unterschiede zwischen den verschiedenen psychoanalytisch begründeten Verfahren ist bei Streeck (2005) und auch bei Reimer und Rüger (2003) zu finden.
} 


\subsubsection{Stand der psychodynamischen Outcome-Forschung}

Im Vergleich zur kognitiv-behavioralen Psychotherapie gibt es für psychodynamische Therapien deutlich weniger randomisierte und kontrollierte Studien, die deren Effektivität bei spezifischen psychischen Störungen untersuchen. Eine dieser wenigen störungsspezifischen Studien konnte jüngst die Wirksamkeit analytischer Langzeitpsychotherapie bei depressiven Störungen, Angststörungen und Persönlichkeitsstörungen belegen (vgl. Jakobsen et al., 2007).

In eine Meta-Analyse von Leichsenring et al. (2006) wurden alle bekannten randomisierten und kontrollierten Studien zur Feststellung der Effektivität von psychodynamischer Psychotherapie bei spezifischen psychischen Störungen einbezogen (vgl. 2.2 in diesem Kapitel). Diese Meta-Analyse liefert erste Hinweise auf die Effektivität dieses Therapieansatzes bei folgenden Störungen: bei depressiver Störung, bei Sozialphobie, bei Generalisierter Angststörung, bei Posttraumatischer Belastungsstörung, bei Borderline Persönlichkeitsstörung, bei Cluster C Persönlichkeitsstörung, bei Bulimia Nervosa, bei somatoformer Störung sowie bei moderater Alkohol- und Opiatabhängigkeit.

\subsubsection{Wirkfaktoren psychodynamischer Psychotherapie}

Nach Luborsky (1995) gibt es drei Wirkfaktoren der psychodynamischen Therapie, wobei jeder Faktor sowohl einen Patienten- als auch einen Therapeutenanteil aufweist.

Der Wirkfaktor Selbsterfahrung und Selbstverständnis wird über die expressiven Behandlungstechniken verwirklicht. Mit Hilfe dieser erlangt der Patient Einsicht in seine wiederkehrenden Konflikte, die seine Probleme aufrechterhalten. Damit er eine Besserung erzielen kann, sollte er ein vertieftes Verständnis seiner selbst erreichen, z.B. über das Wiedererleben seiner konflikthaften Beziehungsprobleme im Hier und Jetzt in der Beziehung zum Therapeuten. Diese Form der Übertragung wird wiederholt mit dem Patienten durchgearbeitet. Dadurch kann er günstigere Lösungen seiner Probleme entwickeln, da er einerseits seine Beziehungsprobleme toleranter sehen („Aufhebung der Verdrängung“) und sich andererseits adaptiver verhalten kann (vgl. Mayman, 1978). Aufgabe des Therapeuten ist es, den Patienten in seinen Fähigkeiten und seinem Bemühen nach einem größeren Verständnis seiner selbst zu unterstützen. Die expressive Arbeit des Therapeuten besteht in einem Zyklus von „Zuhören“, „Verstehen“, „Deuten“ und „erneutem Zuhören“, was als „,interaktives Feedbacksystem zwischen Patient und Therapeut“ (Luborsky, 1995, S. 34) verstanden wird. Ein umfassendes Verstehen des Patienten sollte jedoch in eine supportive Beziehung eingebettet sein. 
Der Wirkfaktor „hilfreiche Beziehung“ wird als wichtigster supportiver Wirkfaktor verstanden. Supportive Interventionen sollen die „Ich-Funktionen“ des Patienten stärken (vgl. Leichsenring et al., 2006). Der Patient sollte die Beziehung zum Therapeuten als hilfreich erleben. Dies ist dann möglich, wenn er den Therapeuten einerseits so wahrnimmt, dass dieser ihm die gewünschte Unterstützung gewährt (Typ I) und wenn er andererseits die Therapie als einen Prozess erlebt, in dem Therapeut und Patient gemeinsam auf die vorher definierten Ziele hinarbeiten (Typ II). Aufgabe des Therapeuten ist es, dem Patienten dies zu ermöglichen, indem er sich an den therapeutischen Kontrakt hält. Dies beinhaltet ein empathisches Verstehen des Patienten. Auch soll er als „Modellperson eines hilfreichen Mitmenschen“ fungieren (vgl. Luborsky, 1995).

Eine Nachhaltigkeit der Behandlungsfortschritte kann erreicht werden, indem der Patient die therapeutischen Fortschritte internalisiert. Dazu übernimmt er, unterstützt vom Therapeuten, die Problemlösungsansätze des Therapeuten und der Therapie für sich. Das Durcharbeiten der Bedeutung des Behandlungsendes ist außerdem besonders zu berücksichtigen.

\subsection{Vergleich der Störungskonzepte von psychodynamischen und kognitiv-behavioralen}

\section{Therapieansätzen}

Psychodynamische Therapieansätze gehen von einem medizinischen Krankheitsmodell aus. Dies bedeutet, dass psychische Abweichungen als personengebundenes Defizit mit biologisch-genetischen Ursachen verstanden werden (vgl. Huf, 1992). Ein Schwerpunkt liegt hier auf der Feststellung der Diagnose. Erst nach Vergabe einer solchen kann das Vorgehen bei der Behandlung festgelegt werden, wobei mit dieser Diagnose Annahmen über die Entstehung und den Verlauf der Störung verbunden sind. Störungen werden hier demnach als Einheiten verstanden. In einem medizinischen Krankheitsmodell wird zwischen Krankheitsund Symptomkomplex unterschieden. Unter Krankheitskomplex werden pathologische Prozesse zusammengefasst, die mit bestimmten psychischen Auffälligkeiten korrelieren. Symptomkomplexe hingegen sind all jene Auffälligkeiten, die zu einem bestimmten Zeitpunkt bei einem bestimmten Menschen vorhanden sind. Die Verbindung zwischen Krankheits- und Symptomkomplex wird durch medizinisches Wissen hergestellt. Das Ergebnis ist eine Diagnose, die der Benennung des Krankheitskomplexes entspricht. Psychodynamische Ansätze fokussieren Veränderungen auf emotionaler und kognitiver 
Ebene und versuchen das Bewusstsein des Patienten hinsichtlich seiner Gefühle zu sich selbst zu stärken (Huf, 1992).

Kognitiv-behaviorale Interventionsansätze gehen von einem bio-psychosozialen Modell (vgl. Huf, 1992) aus. Psychische Störungen werden hier im Kontext von psychologischen, biologischen, ökonomischen und sozialen Variablen gesehen, die sowohl bei der Entstehung als auch bei der Aufrechterhaltung der Störung eine Rolle spielen können. Ihnen liegen demnach nicht einheitliche Krankheitsprozesse zugrunde, sondern individuelle Lebenssituationen, die auch in der Therapie zu berücksichtigen sind. Der diagnostische Prozess ist nicht mit der Diagnosestellung abgeschlossen, stattdessen bedeutet therapeutisches Handeln hier eine ständige Reflexion der Therapieziele und der therapeutischen Maßnahmen bezüglich ihrer Angemessenheit für den jetzigen Zustand des Patienten.

Verhaltenstherapeutische Verfahren versuchen durch die Therapie Veränderungen auf emotionaler, kognitiver, physiologischer und behavioraler Ebene zu erzielen und legen ihren therapeutischen Fokus auf die Beziehung zwischen Gedanken, Gefühlen und Verhalten.

\section{Psychotherapie und Psychotherapieforschung}

\subsection{Psychotherapie-Outcome versus Psychotherapie-Prozess}

Bis Mitte des vergangenen Jahrhunderts wurden psychotherapeutische Behandlungen zunächst angewendet, ohne dass ihre Wirksamkeit empirisch überprüft wurde. Zur Feststellung des „Erfolgs“ der Behandlung wurde allenfalls der Zustand des Patienten vor der Therapie mit dem nach der Therapie verglichen (intraindividuelle Prä-Post-Vergleiche; vgl. Meyer, 1990a). Die genauere Erforschung psychotherapeutischer Methoden und Vorgehensweisen sowie ihrer Effekte setzte sich erst im Kontext von Eysencks (1952) „Angriffen“ auf die Psychotherapie (vgl. Kapitel I) durch („Psychotherapieforschung“).

Innerhalb der Psychotherapieforschung wird zwischen dem, was während der einzelnen Therapiesitzungen, also im Therapieprozess geschieht und den plötzlichen oder langfristigen Veränderungen im Patienten, die durch diese Interventionen erzielt werden konnten, also dem Outcome der Therapie, unterschieden (vgl. Hill \& Lambert, 2004, S. 84; Orlinsky \& Howard, 1987). Entsprechend wird Forschungsarbeit auf diesen beiden Gebieten als PsychotherapieProzess- bzw. Psychotherapie-Outcome-Forschung bezeichnet. Wird nur das Outcome einer Therapie betrachtet, lassen sich keine Rückschlüsse darauf ziehen, was zu den Veränderungen 
im Patienten geführt hat. Die Integration von Befunden aus Prozess- und Outcome-Forschung ist daher wünschenswert und wird im Rahmen dieser Arbeit angestrebt.

In Bezug auf die Veränderungen, die durch eine Psychotherapie bewirkt werden, ist weiterhin zwischen „Outcome“ und „Output" zu unterscheiden. Unter „Output" werden sämtliche Effekte und Veränderungen, die die Therapie z.B. auch in der Familie des Patienten oder im Therapeuten erzielt, subsumiert (vgl. Orlinsky, Roennestad \& Willutzki, 2004). Die Effekte hingegen, die psychotherapeutische Interventionen ausschließlich auf den Patienten ausüben und durch die Veränderungen in diesem angeregt werden, werden als Outcome bezeichnet. Auch die Perspektive, von der aus das Therapieoutcome betrachtet wird, spielt eine bedeutsame Rolle: So beurteilen Patienten selbst es anders als ihre Therapeuten und diese wiederum anders als außenstehende Beobachter. Dies deutet darauf hin, dass es für alle an einer Psychotherapie beteiligten Personen eine unterschiedliche Bedeutung hat. Im Rahmen der vorliegenden Arbeit werden Aspekte des Therapieprozesses mit dem Outcome der Therapie in Verbindung gebracht. Der Therapieprozess wird von unabhängigen Beobachtern erfasst und mit Outcome-Beurteilungen der Patienten und denen unabhängiger Diagnostiker (vgl. Abschnitt 2 in Kapitel III) in Zusammenhang gestellt.

\subsection{Psychotherapieforschung}

Carl Rogers (z.B. 1942 a, b) und seine Arbeitsgruppe, die schon früh auf der Suche nach „Wirkfaktoren der Psychotherapie“ (vgl. 3.5 in diesem Kapitel) waren, gelten heute als Wegbereiter der modernen Psychotherapieforschung.

Eine Reihe von Meta-Analysen (z.B. Smith \& Glass, 1977; Smith et al., 1980; Shapiro \& Shapiro, 1982; Wittmann \& Matt, 1986; Grawe, 1992; Lipsey \& Wilson, 1993) konnte schon relativ früh die generelle Wirksamkeit von Psychotherapie sowohl im kontrollierten als auch im naturalistischen Setting mit einer durchschnittlichen Effektstärke zwischen .60 und .85 belegen (vgl. z.B. Shadish et al., 1997; Grawe, 1992).

Nachdem die generelle Wirksamkeit von Psychotherapie empirisch belegt war, wurde zunehmend die Frage untersucht, welcher der zahlreichen Therapieansätze der wirksamste sei. Dieser Frage wurde und wird mit Hilfe komparativer Outcome-Studien nachgegangen, in denen zwei oder mehr alternative Behandlungsformen miteinander verglichen werden, denen wiederum zumeist unterschiedliche theoretische Konzeptionen zugrunde liegen (Kazdin, 1986). Insgesamt wurden in diesem Zusammenhang vor allem psychodynamische und 
Theoretischer und empirischer Hintergrund

Psychotherapieprozessforschung

interpersonale ${ }^{5}$ mit verhaltenstherapeutischen bzw. kognitiv-behavioralen Ansätzen verglichen, wobei Therapieforscher verschiedene Therapierichtungen immer wieder nach „Gewinnern“ und „Verlierern“ einzuordnen versuchten (Omer \& Dar, 1992).

Eine herausragende Stellung unter den Studien, die Verhaltenstherapie als klaren „Gewinner“ ausmachen konnten, nimmt eine sehr umfangreiche Untersuchung von Grawe et al. (1994) ein. Hier wurde versucht, quasi alle kontrollierten (und publizierten) Psychotherapie-Effektivitätsstudien mit klinischen Populationen bis 1983/84 zu berücksichtigen. Im Zusammenhang mit dieser Studie ist jedoch auch auf die Kritik, die sie erfahren hat, hinzuweisen (z.B. wegen mangelhafter interner Validität oder der großen Überzahl an Studien zur Verhaltenstherapie) ${ }^{6}$. Auch andere Forscher fanden in ihren Studien Belege für die Überlegenheit der Verhaltenstherapie, wie z.B. Giles (1983a, b, 1984) und Svartberg und Stiles (1991).

Wieder andere Forschergruppen konnten trotz der theoretischen Unterschiede zwischen verschiedenen Therapieansätzen keine Effektivitätsunterschiede finden (z.B. Sloane, Staples, Cristol, Yorkston \& Whipple, 1975; Grawe, 1976; Smith \& Glass, 1977; Luborsky et al., 1975; Shapiro et al., 1994; Wampold et al., 1997; Wampold, 2001; Leibing et al., 2005). So berechnete Grissom (1996) einen nichtsignifikanten mittleren Unterschied in den Effektgrößen von $\mathrm{d}=.23$ zwischen verschiedenen Psychotherapierichtungen.

Eine besondere Stellung unter den komparativen Outcome-Studien, die keinen „Gewinner“ ausmachen konnten, nimmt das „Treatment of Depression Collaborative Research Program“ (TDCRP) des „National Institute of Mental Health“(NIMH) ein (z.B. Elkin et al., 1989; Imber et al., 1990; Elkin, 1994). Hier wurde in einer groß angelegten, multizentrischen Studie interpersonale und kognitiv-behaviorale mit psychopharmakologischer Therapie der einfachen, nonbipolaren, nichtpsychotischen Depression miteinander verglichen. Über die gesamte Stichprobe hinweg zeigten sich keine signifikanten Differenzen zwischen den beiden Psychotherapieansätzen untereinander (und auch nicht zwischen den Psychotherapien und der Pharmakotherapie). Diese Studien konnten insgesamt das Äquivalenzparadoxon (vgl. Kapitel I) bzw. das „Dodo-Bird-Verdikt“ („Everybody has won and all must have prizes.“; vgl. Rosenzweig, 1936) bestätigen.

\footnotetext{
${ }^{5}$ Zum Verhältnis von Interpersonaler Therapie zu psychodynamischen bzw. kognitiv-behavioralen Ansätzen siehe z.B. Blagys und Hilsenroth (2000) oder die dazu eher konträren Befunde von Ablon und Jones (2002).

${ }^{6}$ Im Rahmen dieser Arbeit ist eine Diskussion dieser Studie jedoch nicht möglich. Der interessierte Leser sei z.B. auf Tschuschke, Kächele \& Hölzer (1994), Tschuschke et al. (1998), Leichsenring (1996) oder Grawe (1998) verwiesen.
} 
In einer aktuellen Untersuchung dazu zogen Leichsenring et al. (2006) als Datenbasis alle bis zum Oktober 2005 publizierten systematischen Reviews aus der Psychotherapie-OutcomeForschung, die evidenzbasierte Methoden (vgl. Chambless \& Ollendick, 2001) verwendet hatten, heran. Es wurden ausschließlich Studien, die mit randomisierten und kontrollierten Stichproben arbeiteten, berücksichtigt. Neben systematischen Untersuchungen und MetaAnalysen wurden auch Originalstudien, die diesen Ein- bzw. Ausschlusskriterien genügen konnten, einbezogen. Alle Behandlungen wurden mit erwachsenen Patienten durchgeführt. Leichsenring et al. (2006) fanden, dass in einigen Studien die Verhaltenstherapie der psychodynamischen Therapie überlegen war (z.B. bei Shapiro et al., 1994; Garner et al., 1993), in anderen hingegen nicht (z.B. bei Barkham et al., 1996; Bögels, Wijts \& Sallaerts, 2003; Brom, Kleber \& Defares, 1989; Sandahl, Herlitz, Ahlin \& Rönnberg, 1998; Svartberg, Stiles \& Seltzer, 2004; Woody, Luborsky, McLellan \& O’Brien, 1990).

Lambert und Ogles (2004) erklärten diese insgesamt widersprüchliche Befundlage mit der eingeschränkten externen Validität kontrollierter Studien, mit der Behandlung von eher leichten Fällen und mit der Verwendung von Erfolgsmaßen, bei denen Reaktivitätseffekte wahrscheinlich sind. Auch die so genannte ,allegiance“ (Identifikation des Forschers mit dem untersuchten Verfahren) soll eine Rolle spielen. Nach Luborsky et al. (1999) korrelieren Therapieergebnisse mit bis zu .85 mit der ,allegiance“.

Insgesamt scheint es wenig sinnvoll, generelle „Verlierer“ oder „Gewinner“ identifizieren zu wollen, da mit großer Sicherheit der Therapieerfolg durch die Interaktion verschiedener Faktoren bestimmt wird, von denen der theoretische Hintergrund der Therapie sicher nur einer, und vielleicht auch gar nicht der wichtigste ist (vgl. 3. in diesem Kapitel; Stiles et al., 1986). Daher wird in der vorliegenden Untersuchung ein weiterführender Ansatz propagiert, dessen Ziel die Untersuchung und Identifikation von Therapieprozessfaktoren, die den Erfolg moderieren, ist. Über den Vergleich von Therapieprozessen werden Aussagen darüber, ob und worin Therapien sich überhaupt unterscheiden, erst möglich (vgl. Leichsenring et al., 2006).

\section{Psychotherapieprozessforschung}

\subsection{Methodische Anforderungen an die Psychotherapieprozessforschung}

Psychotherapieprozessforschung untersucht die Beziehung von Therapieverlauf und Therapieergebnis und wird daher von der Frage „Welche therapeutischen Prozesse führen zu welchen Ergebnissen?" geleitet. In der aktuellen Psychotherapieprozess-Forschung wird über einen Vergleich von mindestens zwei unterschiedlichen Behandlungsansätzen u.a. untersucht, 
welche (unterschiedlichen und/oder gemeinsamen) Interventionen des Therapeuten und Verhaltensweisen des Patienten, welche (unterschiedlichen und/oder gemeinsamen) PatientTherapeut-Interaktionen und welche (unterschiedlichen und/oder gemeinsamen) im Therapieprozess fokussierten Themen die unterschiedlichen Therapieprozesse jeweils konstituieren. Zur Untersuchung von Veränderungen innerhalb einer Therapie sollte nach Möglichkeit der gesamte Therapieprozess von seinen Anfängen bis zu seinem Ende herangezogen und alle Aspekte, die zu therapeutischen Veränderungen führen könnten, erfasst und auf ihren Zusammenhang mit dem Outcome geprüft werden.

Die moderne Psychotherapieprozessforschung profitiert von zwei wesentlichen Entwicklungen. Zum einen von der Einführung psychometrischer Methoden zur Erfassung von Ereignissen, Erfahrungen und Erlebnissen von Patient und Therapeut in der Therapie mittels regelmäßig im Verlauf der Therapie eingesetzter Ratingskalen und Fragebögen (vgl. Thurstone \& Chave, 1929) und zum anderen von den verbesserten Möglichkeiten der audiovisuellen und digitalen Dokumentation von Therapiesitzungen (z.B. Laswell, 1935, 1936, 1937; Rogers, 1942a, 1942b).

Bei der Untersuchung des Psychotherapie-Prozesses kann zwischen qualitativen und quantitativen Untersuchungsmethoden unterschieden werden (vgl. Hill \& Lambert, 2004). Bei der quantitativen Prozessforschung werden bereits erprobte Messinstrumente verwendet, deren Ergebnisse mit Hilfe verschiedener statistischer Methoden zusammengefasst und analysiert werden (vgl. Hill \& Lambert, 2004). Zur statistischen Analyse der Daten werden deskriptive und korrelative Ansätze sowie sequentielle Analysen angewendet (vgl. Hill \& Lambert, 2004). Bei deskriptiven Ansätzen werden Häufigkeiten der Prozessvariablen angegeben, bei korrelativen Ansätzen wird die Häufigkeit des Auftretens einer bestimmten Prozessvariable mit einem Outcome-Maß in Beziehung gesetzt. Sequentielle Analysen hingegen untersuchen die direkten Effekte verschiedener Ereignisse im Therapieprozess. Hier werden eher Interaktionen, d.h. z.B. welches therapeutische Verhalten führt zu welchen Reaktionen des Patienten, untersucht (z.B. über Varianzanalysen). Bereits etablierte Messinstrumente zu verwenden, bietet den Vorteil, dass ihre Validität und Reliabilität schon überprüft wurde. Auch ist eine Vergleichbarkeit zwischen den Befunden verschiedener Studien möglich, in denen die gleichen Messinstrumente eingesetzt wurden (vgl. Hill \& Lambert, 2004). Ein möglicher Nachteil ist darin zu sehen, dass die bereits existierenden Messinstrumente nicht immer exakt das erfassen, was mit der jeweiligen Studie eigentlich untersucht werden sollte. 
Bei der qualitativen Prozessforschung hingegen werden zunächst Daten gesammelt, ohne dass dazu genaue Kriterien vorgegeben werden (z.B. mit Hilfe eines Interviews). Erst in einem zweiten Schritt werden aus diesen so gewonnenen Daten Kategorien gebildet, wobei dies v.a. aufgrund inhaltlicher Gesichtspunkte geschieht (vgl. Hill \& Lambert, 2004). Vorteil dieser Vorgehensweise ist, dass damit auch nur selten auftretende Phänomene, die mit quantitativer Forschung evtl. nicht beobachtet werden könnten, untersucht werden. Nachteil ist die Schwierigkeit, diese Ergebnisse dann über mehrere Studien hinweg zusammenzufassen und so vergleichbar zu machen (Hill \& Lambert, 2004). Auch ist die Validität und die Reliabilität der Daten und Beobachtungen nicht immer eindeutig zu bestimmen. Im Rahmen der vorliegenden Arbeit werden quantitative Methoden der Prozessforschung benutzt.

Hinsichtlich des Therapieprozesses ist zwischen verschiedenen Dimensionen zu unterscheiden, zu deren Operationalisierung jeweils eigene Messinstrumente konzipiert werden. Elliott (1991) z.B. unterscheidet fünf Dimensionen des Therapieprozesses. Unter Beobachtungs- oder Bewertungsperspektive versteht er den Standpunkt, von dem aus die Therapie beschrieben wird, also aus Sichtweise des Patienten, des Therapeuten oder eines Beobachters. Unter Fokus der Bewertung wird die jeweils untersuchte Person bzw. der jeweils untersuchte Aspekt verstanden (z.B. der Patient, der Therapeut, der Supervisor oder die Dyade/das System). Eine weitere Dimension des Therapieprozesses sind Aspekte des Prozesses. Hier geht es darum, welcher Teil des Prozesses untersucht wird (z.B. der Typ des Prozesses, also offenes Verhalten des Therapeuten oder seine Absichten, der Inhalt des Gesprächs, der Stil oder die Art der jeweils sprechenden Person oder die Qualität der Interaktion). Eine weitere Dimension ist das Analyselevel. Darunter wird die untersuchte Einheit des Therapieprozesses verstanden. Es wird zwischen mikroprozessualen (z.B. einzelne Wörter, Phrasen, Sätze oder Gedankeneinheiten) und makroprozessualen Einheiten (z.B. Sitzungen, Behandlungsabschnitte oder die gesamte Therapie) unterschieden (vgl. Hill \& Lambert, 2004). Elliott (1991) führt als letzte Dimension die sequentielle Abfolge des Therapieprozesses an. Darunter versteht er die Sequenz des Therapieprozesses, die untersucht wird (z.B. die inhaltliche, der Kontext, in dem der Prozess abläuft, oder das Outcome). Hill und Lambert (2004) nennen außerdem die theoretische Basis der Messung (z.B. atheoretisch, psychodynamisch oder behavioral), den Typ der Messung (Intervallskalen, nominale Kategorien oder Q-Sorts), das Inferenzlevel sowie das für die Bewertungen verwendete Stimulusmaterial (d.h. direkte Beobachtung, Transkript oder Audio- oder Videokassette) als weitere Therapieprozessdimensionen. Im Hinblick auf die jeweils spezifische Fragestellung 
sind dann die zu untersuchenden Dimensionen zu spezifizieren (Greenberg, 1986) und zu operationalisieren (vgl. Hill \& Lambert, 2004).

Um mit Hilfe von Prozessforschung aussagekräftige Befunde zu erhalten, sind einige methodische Aspekte für die Durchführung der Therapien zu beachten. Die behandelnden Therapeuten sollten für den jeweiligen Therapieansatz sorgfältig geschult werden und die Therapien anhand von Manualen durchführen (vgl. Chambless \& Hollon, 1998). Die Benutzung eines Manuals soll zu einer Standardisierung des Vorgehens führen (vgl. Kendall et al., 2004). Dies soll ggf. eine Replikation des Studienvorgehens und der Studienergebnisse ermöglichen (Yeaton \& Sechrest, 1981). Mittels Supervision soll u.a. gewährleistet werden, dass die Therapien wie vorgesehen implementiert werden. Die Manualisierung ermöglicht die Überprüfung der „adherence“ (vgl. Moncher \& Prinz, 1991, S. 248), d.h. der manualgetreuen Umsetzung der Therapien in die Praxis (vgl. Hogue et al., 1998). „Adherence“ wiederum korreliert hoch mit dem Erfolg der Therapie. Nur wenn die ,,adherence“ sichergestellt ist, können ggf. vorhandene Effektivitätsunterschiede zwischen den Therapieansätzen intern valide interpretiert werden (vgl. Reinecker, 2000). Weiterhin sollten die Therapien bei einem ähnlichen Patientenkollektiv mit vergleichbaren Diagnosekategorien (z.B. nach DSM-IV, APA, 1994 oder ICD-10, WHO, 1993) durchgeführt wurden. Goldfried und Wolfe (1996) bezeichnen diese Form von Forschung als Generation III Forschung. Die Berücksichtigung dieser Forderungen trägt ganz entscheidend zur Validität der Ergebnisse bei.

In der vorliegenden Studie wurden deshalb ausschließlich Patienten mit der Diagnose einer Generalisierten Angststörung behandelt. Beide Therapien, die tiefenpsychologisch fundierte und die kognitiv-behaviorale, wurden manualisiert durchgeführt. Außerdem wurde versucht, die Implementierung der Therapien durch ein Therapiemanual, ein Manualtraining und durch begleitende Supervision sicherzustellen. Es soll untersucht werden, in welchen Prozessaspekten sich diese beiden Therapien ähneln bzw. unterscheiden, in welcher Beziehung die Prozessmerkmale zum Outcome stehen und ob sie damit ggf. als Wirkfaktoren (vgl. 3.5) zu interpretieren sind. Der Therapieprozess wird hier aus der Perspektive eines unabhängigen Beobachters makroprozessual, d.h. unter Berücksichtigung einer kompletten Therapiestunde, untersucht. Eine vollständige Sitzung stellt einen naturalistischen Zeitrahmen dar, der für den Forscher einen praktischen Nutzen und für den Kliniker und den Patienten eine inhärente Bedeutung hat. Die Beurteilung einer gesamten Therapiesitzung hat den Vorteil, dass bestimmte Ereignisse in ihren Kontext und die zeitliche Gliederung eingeordnet werden können. Auch verschiedene Phasen der Behandlung können so verglichen werden. In 
dieser Studie wird das offene und sichtbare Verhalten des Patienten und des Therapeuten sowie ihre Interaktion und der Inhalt des Gesprächs untersucht. Mit Hilfe eines Q-SortVerfahrens, dem Psychotherapie-Prozess-Q-Sort (PQS ${ }^{7}$, Jones, 1985, 2000), das atheoretisch konstruiert wurde, werden Audiomitschnitte der Therapiesitzungen beurteilt. Aufgrund klarer Vorgaben im PQS-Manual für die Vorgehensweise bei der Beurteilung und aufgrund der Fokussierung von offen in der Therapiestunde sichtbaren Eigenschaften ist das Inferenzlevel niedrig.

\subsection{Das Psychotherapie Prozess Q-Sort (Jones, 2000)}

Q-Sort-Verfahren gehören zur Q-Methodologie und bieten die Möglichkeit, den Therapieprozess und damit qualitative Daten quantitativ zu beschreiben und zu untersuchen. Bei der Q-Methode werden zwar ebenfalls Faktorenanalysen durchgeführt, statt Tests oder Variablen werden hier jedoch Personen miteinander korreliert. Um den Unterschied zur klassischen R-Faktorenanalyse zu verdeutlichen, bezeichnete ihr Entwickler, der Psychologe und Physiker R. Stephenson (1951, 1953), diese Methode daher als Q-Methode. Der QMethodologie liegen sowohl ein spezifisches Erhebungsverfahren (Q-sort-Technik) als auch ein spezifisches Auswertungsverfahren (Q-Technik) zugrunde. Bei der Q-sort-Technik werden Karten, auf denen Statements, einzelne Wörter oder auch Bilder gedruckt sind, auf einer Rating-Skala von z.B. „zutreffend“ bis ,nicht zutreffend“ in Relation zueinander beurteilt. Diese Karten beschreiben die jeweils relevanten Aspekte des Untersuchungsthemas und bilden so das Q-set. Ein Q-Sort-Verfahren ist damit ein Rangordnungsverfahren. Die QTechnik beschreibt die Korrelation der einzelnen Q-sorts der befragten Personen miteinander. Mit Hilfe dieser Korrelationen und Faktorenanalysen können Typen gebildet oder Einzelfälle systematisch analysiert werden (vgl. Müller \& Kals, 2004).

Da es kein Standard-Q-set gibt, sind für jeden Untersuchungsbereich eigene Q-sets mit passenden Statements, Wörtern oder Bildern (Items) zu konzipieren, die die jeweils kritischen Dimensionen des Untersuchungsgegenstands, d.h. die bedeutsamen psychologischen oder verhaltensbezogenen Merkmale eines Individuums oder einer Situation, so vollständig wie möglich erfassen sollten.

Zur Beurteilung eines Q-sorts stehen zwei Möglichkeiten zur Verfügung: entweder wird es den Beurteilern freigestellt, wie häufig sie welche Kategorie zur Beurteilung benutzen

\footnotetext{
${ }^{7}$ Bei wiederholter Nennung des gleichen Verfahrens wird zur besseren Lesbarkeit in der Regel auf die ausgeschriebene Bezeichnung sowie auf die Autorenangabe verzichtet. Angegeben wird dann jeweils nur noch die bei der Erstnennung eingeführte Abkürzung.
} 
(,unforced Q-sort“") oder es wird ihnen vorgeschrieben, wie häufig sie die Kategorien mit den Items belegen dürfen (,forced Q-sort“) ${ }^{8}$. Dann beschreibt die Häufigkeit, mit der die Karten in die Kategorien einsortiert werden müssen, (meist) eine Normalverteilung.

Das PQS ist ein solches forced Q-sort. Es erhebt den Anspruch, alle im Therapieprozess relevanten Variablen detailliert erfassen zu können. Mit dem PQS soll es möglich sein, den Psychotherapieprozess systematisch zu beschreiben, zu klassifizieren und zu beurteilen. Das PQS ist ein ipsatives Q-Sort-Rating-Verfahren. Dies bedeutet, dass nicht Vergleiche zwischen Individuen oder Skalen gezogen werden („Ist Herr A ängstlicher als Herr B?“), sondern dass eine relationale Statementstruktur erzeugt und auch intendiert wird (vgl. Müller \& Kals, 2004). Die Items innerhalb eines Falles werden demnach nicht unabhängig voneinander, sondern in Relation zueinander beurteilt („Ist Herr A eher ängstlich oder eher aggressiv?“). Mit Hilfe des PQS können sowohl nomothetisch orientierte Forschungen als auch idiographische Einzelfallanalysen durchgeführt werden.

Jones (1985) entschied sich mit dem PQS für eine molare Vorgehensweise (vgl. Lambert \& Hill, 1994, S. 96), indem er sowohl die Haltung, das Verhalten oder Erleben des Patienten, die Interventionen und Haltungen des Therapeuten sowie Merkmale ihrer Interaktion beurteilen lässt. Die Items des PQS wurden aus mehreren Hundert potentiell bedeutsamen Merkmalen und Eigenschaften des Therapieprozesses hinsichtlich ihrer Eindeutigkeit und Wichtigkeit für die Therapie ausgewählt. Sie stammten einerseits aus bereits existierenden Inventaren zu Prozessvariablen und wurden andererseits von forschungsorientierten Klinikern neu konstruiert. Therapeuten verschiedener Therapieansätze (klienten-zentriert, Gestalt-, rational-emotiv, psychodynamisch und kognitiv-behavioral) wendeten in Pilotstudien diese vorläufige Form des PQS an, um Video- und Audioaufzeichnungen von Therapiestunden zu beurteilen. Neue Items wurden dann hinzugefügt, wenn die Therapeuten feststellten, dass mit Hilfe der vorhandenen Items die Therapiestunde nicht ausreichend beschrieben werden konnte. Items wurden eliminiert, wenn sie über viele Personen und Therapiestunden hinweg

\footnotetext{
${ }^{8}$ Brown (1995) sieht in einem „forced Q-sort“ den Vorteil, dass Personen, die sich bisher weniger bewusst über ihre Präferenzen zum erfragten Bereich waren, zu einer Entscheidung gezwungen werden und sich dadurch ihre eigene Position manifestiert. Besonders bedeutsame Items erhalten so die stärkste Gewichtung. Göttert und Asendorpf (1989) heben hervor, dass die gesamte Skala zur Beurteilung genutzt werden muss und daher Antworttendenzen (z.B. „Zentrale Tendenz“) und negative oder positive Halo-Effekte (vgl. Jones, Cumming \& Pulos, 1993) nahezu völlig auszuschließen sind. Statistische Analysen werden erleichtert, da alle Q-sorts die gleiche Verteilung haben. Roston, Lee und Vaillant (2005) sehen einen weiteren Vorteil darin, dass bei jeder Person, die beurteilt werden soll, die gleichen Kriterien für ihre Beurteilung angelegt werden. Zu der z.T. sehr kontrovers geführten Debatte hinsichtlich der unterschiedlichen Verteilungsverfahren sei auf Bolland (1985) oder Cottle und McKeown (1980) verwiesen.
} 
wenig Variation, eine hohe Korrelation untereinander oder eine niedrige Interrater-Reliabilität zeigten (vgl. Jones, Hall \& Parke, 1991). Die aktuell gültige Version des PQS besteht aus 100 Items (Jones, 2000), die in einem Manual genau beschrieben werden und auf einer neunstufigen Skala von „extrem uncharakteristisch“ über „,neutral, unwichtig“ bis „extrem charakteristisch“ zu beurteilen sind. Die Items sind mit jeweils einem Beispiel versehen, das veranschaulicht, wie beim Codieren vorzugehen ist. So sollen unterschiedliche Interpretationen der Items verhindert werden. Die Q-Items beschreiben offen in der Therapiestunde sichtbare Verhaltensweisen des Patienten, therapeutische Interventionen und Haltungen sowie Merkmale der Patient-Therapeut-Interaktion. So beurteilt ein Rater z.B. nicht, ob der Patient „Abwehrmechanismen“ zeigt, d.h. z.B. ein bestimmtes Thema verdrängt, sondern nur, ob der Therapeut „Abwehrinterpretationen“ macht oder nicht, d.h. ob der Therapeut z.B. auf eine solche Verdrängung hinweist oder nicht.

\subsection{Befunde aus der Prozessforschung mit dem PQS}

Die Arbeitsgruppe um E. Jones untersuchte mit Hilfe des PQS Psychotherapieprozesse unterschiedlicher Länge und theoretischer Fundierung darauf, ob sich Unterschiede in den therapeutischen Interventionen, in den Patient-Therapeut-Interaktionen und in den Verhaltensweisen des Patienten in der Therapie zeigten. Auch erforschten sie damit potentielle Wirkfaktoren der jeweiligen Psychotherapien und versuchten diese in unspezifische und spezifische Wirkfaktoren (vgl. Kapitel I) einzuordnen. Sie führten sowohl Einzelfallstudien (vgl. Jones \& Windholz, 1990; Jones, Ghannam, Nigg \& Dyer, 1993) als auch vergleichende Gruppenuntersuchungen (vgl. Jones, Cumming \& Horowitz, 1988; Jones \& Pulos, 1993; Ablon \& Jones, 1998, 1999, 2002) durch.

In einer Studie von Jones et al. (1988) wurde der Zusammenhang zwischen Prozessvariablen und Outcome bei 40 Patienten mit einer Posttraumatischen Belastungsstörung untersucht. Die Patienten wurden mit einer zwölf Stunden dauernden psychodynamischen Psychotherapie behandelt, von der jeweils die fünfte Sitzung von je drei unabhängigen Beurteilern mit dem PQS beurteilt wurde. Es zeigte sich eine Interaktion von Interventionen mit bestimmten Patientenmerkmalen. So erwies sich bei den weniger schwer gestörten Patienten ein expressiver/Angst provozierender Ansatz überlegen. Bei diesem stehen emotionale und interpersonelle Aspekte im Vordergrund der Behandlung. Schwerer gestörte Patienten hingegen profitierten stärker von einem supportiven/Angst unterdrückenden Ansatz. Die Therapie hat hier einen externalen Fokus, statt emotionaler 
Konflikte werden eher reale Probleme bearbeitet (vgl. Snifeos, 1972; Wallerstein, 1986; vgl. a. 1.2.2 in diesem Kapitel). Demnach existiert keine einfache direkte Verbindung zwischen Prozess und Outcome, sondern eher eine Interaktion multipler Einflüsse.

Jones und Pulos (1993) verglichen die Therapieprozesse von 32 mit kognitiv-behavioraler Therapie behandelten mit 30 mit psychodynamischer Therapie behandelten Patienten mit jeweils unterschiedlichen Störungsbildern. Dazu wurden jeweils die erste, fünfte und 14. Sitzung mit dem PQS beurteilt. Die beiden effektiven Therapien unterschieden sich in 57 der 100 PQS-Items bedeutsam voneinander, v.a. in den Merkmalen, die therapeutische Interventionen oder die Haltung des Therapeuten beschreiben. Keine bedeutsamen Differenzen fanden sich hingegen in den Prozessmerkmalen, die das Verhalten oder den emotionalen Zustand des Patienten in der Therapie betreffen (bei 26 von 43 Items des PQS). Eine Faktorenanalyse über diesen Datensatz erbrachte vier Faktoren, die insgesamt $42 \%$ der Varianz in den PQS-Ratings erklären konnten. Der erste dieser Faktoren vereinigte typische Techniken und Eigenschaften psychodynamischer Therapie in sich, ein weiterer typische Techniken und Eigenschaften kognitiv-behavioraler Therapie. Der dritte Faktor konnte am besten mit „Widerstand des Patienten“ und der vierte Faktor mit „negativer Affekt des Patienten“ beschrieben werden. Im Anschluss an die Faktorenanalyse wurde der Zusammenhang der Faktoren mit dem Outcome untersucht, wobei angenommen wurde, dass hohe Scores auf dem Faktor ,psychodynamische Technik“ mit einem positiven Outcome in den psychodynamischen Therapien und hohe Scores auf dem Faktor „kognitiv-behaviorale Technik" mit einem positiven Outcome in den kognitiv-behavioralen Therapien korrelieren würden. Überaschenderweise zeigte sich dieses Ergebnis jedoch nicht. Der Faktor „psychodynamische Technik“ korrelierte signifikant mit Verbesserungen bei Patienten, die kognitiv-behavioral behandelt wurden, während der Faktor „kognitiv-behaviorale Technik“ hier überhaupt keinen Zusammenhang mit dem Outcome aufwies. In den psychodynamischen Therapien zeigte sich ein Trend zu einem Zusammenhang mit dem Faktor „psychodynamische Technik“ sowie ein signifikant negativer Zusammenhang mit dem Faktor „kognitiv-behaviorale Technik“. Dieses Ergebnis interpretieren die Autoren so, dass der Erfolg einer Therapie umso größer wird, je mehr psychodynamische Techniken der Therapeut einsetzt. Dies gilt auch für kognitiv-behaviorale Therapien. Demnach verwenden auch kognitiv-behavioral orientierte Therapeuten psychodynamische Techniken, wobei genau diese Strategien die Veränderungen im Patienten forcieren. Allerdings sind die Befunde dieser Untersuchung kritisch zu sehen, u.a. weil die Hälfte der Patienten neben der kognitiv- 
behavioralen zusätzlich mit einer psychopharmakologischen Therapie behandelt wurde und keine Randomisierung stattfand. Dennoch konnten diese überraschenden Befunde in einer weiteren Studie (Ablon \& Jones, 1998) repliziert werden. Hier wurden zunächst Prototypen als Standards einer typisch psychodynamischen bzw. kognitiv-behavioralen Behandlung entwickelt, indem Experten ihrer jeweiligen Therapierichtung (entweder führende Theoretiker und/oder sehr erfahrene Praktiker) gebeten wurden, eine Frageform des PQS zu beantworten. Jedes der 100 Items des PQS wurde von 11 Experten der psychodynamischen und 10 Experten der kognitiv-behavioralen Therapie daraufhin beurteilt, wie typisch es ihrer Ansicht nach für eine ideal durchgeführte Therapiestunde gemäß ihrer theoretischen Orientierung ist. Die Items waren auf einer Skala von „1 = extrem uncharakteristisch“ bis „, $9=$ extrem charakteristisch“ zu beurteilen. Außerdem wurden die Experten gebeten, weitere Items hinzuzufügen, falls ihrer Meinung nach wichtige Aspekte des jeweiligen Therapieansatzes fehlten, was jedoch nicht der Fall war. Es wurden Korrelationen zwischen den Ratings der Experten berechnet. Bei einer Faktorenanalyse ergaben sich zwei Faktoren mit Eigenwerten über Eins, die insgesamt fast 70 \% der Varianz in den Ratings der Experten erklären konnten. Die Ratings aller 11 psychodynamisch orientierten Experten luden v.a. auf Faktor 1 (,psychodynamische Technik“"), die aller 10 kognitiv-behavioral orientierten Experten v.a. auf Faktor 2 (,kognitiv-behaviorale Technik“"). Diese Ergebnisse sprechen dafür, dass die psychodynamischen und die kognitiv-behavioralen Therapieexperten tatsächlich jeweils nur ein relativ homogenes und vom jeweils anderen Therapiekonzept klar abgrenzbares eigenes Konzept eines idealen Therapieprozesses haben. Im Anschluss wurden lineare Regressionen berechnet, um den Anteil, den jedes Item des PQS an den beiden Faktoren hat, bestimmen zu können (Bestimmung der Faktorwerte). Die Items mit den höchsten Faktorwerten können den Faktor am besten, die mit den niedrigsten ihn am schlechtesten beschreiben. Tabelle 1 und 2 zeigen, welche Faktoren die beiden Therapieansätze jeweils am besten beschreiben können.

Die so erstellten Prototypen wurden nun mit PQS-Ratings, die zuvor von verschiedenen Therapien erstellt wurden, korreliert. Jeweils mindestens zwei Rater hatten dazu die Sitzungstranskripte der 1., 5. und 14. Stunde von entweder einer psychodynamischen Therapie bei $\mathrm{N}=30$ Patienten mit verschiedenen Diagnosen oder von einer kognitivbehavioralen Therapie bei $\mathrm{N}=32$ Patienten mit einer unipolaren Depression beurteilt. Außerdem beurteilten jeweils drei Rater die 5. Stunde einer weiteren psychodynamischen Therapie bei $\mathrm{N}=38$ Patienten mit einer Posttraumatischen Belastungsstörung (PTSD). 


\section{Theoretischer und empirischer Hintergrund}

Psychotherapieprozessforschung

Tabelle 1: Rangreihe der Q-Items anhand der Faktorwerte „psychodynamische Technik“.

\begin{tabular}{|l|l|l|}
\hline $\begin{array}{l}\text { PQS- } \\
\text { Code }\end{array}$ & Beschreibung des Items & $\begin{array}{l}\text { Faktor- } \\
\text { wert }\end{array}$ \\
\hline 90 & Träume und Phantasien des Patienten werden besprochen. & 1,71 \\
\hline 93 & Der Therapeut ist neutral. & 1,57 \\
\hline 36 & Der Therapeut weist auf Abwehrmechanismen des Patienten hin. & 1,53 \\
\hline 100 & $\begin{array}{l}\text { Der Therapeut stellt Verbindungen zwischen der therapeutischen Beziehung und anderen } \\
\text { Beziehungen des Patienten her. }\end{array}$ & 1,47 \\
\hline 6 & Der Therapeut ist einfühlsam, auf den Patienten eingestellt, empathisch. & 1,46 \\
\hline 67 & $\begin{array}{l}\text { Der Therapeut interpretiert verdrängte oder unbewusste Wünsche, Gefühle oder } \\
\text { Überzeugungen. }\end{array}$ & 1,43 \\
\hline 18 & Der Therapeut vermittelt Wertschätzung. & 1,38 \\
\hline 32 & Der Patient gewinnt ein neues Verständnis oder Einsicht. & 1,32 \\
\hline 98 & Die therapeutische Beziehung ist ein Fokus des Gesprächs. & 1,28 \\
\hline 46 & Der Kommunikationsstil des Therapeuten ist klar und verständlich. & 1,24 \\
\hline 50 & Der Therapeut lenkt die Aufmerksamkeit auf Gefühle, die der Patient für inakzeptabel hält. & 1,17 \\
\hline 11 & Sexuelle Gefühle und Erfahrungen werden besprochen. & 1,12 \\
\hline 82 & $\begin{array}{l}\text { Der Therapeut benennt das Verhalten des Patienten in der Stunde auf eine Art und Weise, } \\
\text { die bisher nicht explizit wahrgenommene Aspekte dieses Verhaltens deutlich werden lässt. }\end{array}$ & 1,12 \\
\hline 35 & Das Selbstbild ist ein Fokus des Gesprächs. & 1,11 \\
\hline 91 & Erinnerungen oder Rekonstruktionen der Kindheit sind Gegenstand des Gesprächs. & 1,08 \\
\hline 92 & $\begin{array}{l}\text { Gefühle oder Wahrnehmungen des Patienten werden mit Situationen oder Verhalten in der } \\
\text { Vergangenheit verknüpft. }\end{array}$ & 1,05 \\
\hline 62 & $\begin{array}{l}\text { Der Therapeut benennt ein sich in der Erfahrung oder dem verhalten des Patienten } \\
\text { wiederholendes Thema. }\end{array}$ & 0,95 \\
\hline 3 & Die Äußerungen des Therapeuten zielen darauf, dem Patienten das Erzählen zu erleichtern. & 0,92 \\
\hline 79 & $\begin{array}{l}\text { Der Therapeut spricht eine Veränderung in der Stimmung oder dem Affekt des Patienten } \\
\text { an. }\end{array}$ & 0,88 \\
\hline 22 & Der Therapeut fokussiert die Schuldgefühle des Patienten. & 0,87 \\
\hline
\end{tabular}

Tabelle 2: Rangreihe der Q-Items anhand der Faktorwerte „kognitiv-behaviorale Technik“.

\begin{tabular}{|l|l|l|}
\hline $\begin{array}{l}\text { PQS- } \\
\text { Code }\end{array}$ & Beschreibung des Items & $\begin{array}{l}\text { Faktor- } \\
\text { wert }\end{array}$ \\
\hline 38 & $\begin{array}{l}\text { Es werden spezifische Aktivitäten oder Aufgaben besprochen, die der Patient außerhalb } \\
\text { der Sitzungen in Angriff nehmen soll. }\end{array}$ & 1,93 \\
\hline 30 & Die Diskussion ist um kognitive Themen zentriert, d.h. Gedanken und Überzeugungen. & 1,68 \\
\hline 4 & Die Therapieziele des Patienten werden besprochen. & 1,51 \\
\hline 85 & $\begin{array}{l}\text { Der Therapeut ermuntert den Patienten, neue Verhaltensweisen im Umgang mit anderen } \\
\text { auszuprobieren. }\end{array}$ & 1,49 \\
\hline 17 & Der Therapeut kontrolliert die Interaktion aktiv. & 1,45 \\
\hline 45 & Der Therapeut nimmt eine supportive Haltung ein. & 1,43 \\
\hline 23 & Das Gespräch hat einen spezifischen Fokus. & 1,38 \\
\hline 31 & Der Therapeut fragt nach mehr Information oder ausführlicherer Schilderung. & 1,37 \\
\hline 69 & Die aktuelle oder kurz zurückliegende Lebenssituation des Patienten wird besprochen. & 1,35 \\
\hline 27 & Therapeut gibt explizit Ratschläge und Anleitungen. & 1,32 \\
\hline 80 & Der Therapeut bietet eine Erfahrung oder ein Ereignis aus einer anderen Perspektive an. & 1,28 \\
\hline 86 & Der Therapeut ist zuversichtlich oder selbstsicher. & 1,21 \\
\hline 37 & Der Therapeut verhält sich ,,lehrerhaft“. & 1,17 \\
\hline 73 & Der Patient fühlt sich der therapeutischen Arbeit verpflichtet. & 1,14 \\
\hline 57 & Der Therapeut erläutert das Rational seiner Technik oder seines Behandlungsansatzes. & 1,13 \\
\hline 88 & Der Patient bringt relevante Themen und Material zur Sprache. & 1,09 \\
\hline 72 & Der Patient versteht das Wesen der Therapie und was von ihm erwartet wird. & 1,08 \\
\hline 95 & Der Patient fühlt sich unterstützt. & 1,06 \\
\hline 28 & Der Therapeut nimmt den therapeutischen Prozess richtig wahr. & 1,05 \\
\hline 48 & Der Therapeut ermutigt den Patienten zur Unabhängigkeit im Handeln oder Denken. & 1,02 \\
\hline
\end{tabular}


In der Stichprobe der kognitiv-behavioral behandelten Patienten zeigte sich ein signifikanter Unterschied zwischen den Korrelationen der PQS-Ratings mit den beiden Prototypen. Während die PQS-Ratings mit dem kognitiv-behavioralen Prototyp hoch positiv korrelierten, zeigten sich zwischen den PQS-Ratings und dem psychodynamischen Prototyp nur niedrige negative Korrelationen. Bei den 30 psychodynamisch behandelten Patienten mit verschiedenen Störungsbildern unterschieden sich die Korrelationen der PQS-Ratings mit den beiden Prototypen nicht signifikant voneinander. Beide Korrelationen sind als hoch einzuordnen, wenngleich die Korrelation mit dem psychodynamischen Prototyp auch etwas höher war als die mit dem kognitiv-behavioralen. In der Stichprobe der psychodynamisch behandelten Patienten mit PTSD zeigte sich ebenfalls kein signifikanter Unterschied in den Korrelationen der PQS-Ratings mit den beiden Prototypen. Kognitive Verhaltenstherapeuten setzten demnach kaum psychodynamische Techniken ein, diese Therapien ähnelten stark dem kognitiv-behavioralen Prototyp. Psychodynamische Therapeuten hingegen wendeten in ihren Therapien sowohl psychodynamische als auch kognitiv-behaviorale Techniken an, entsprechend ähnelten die psychodynamischen Psychotherapien beiden Prototypen (vgl. Ablon \& Jones, 2002). Die Korrelationen der PQS-Ratings mit den beiden Prototypen wurden dann mit verschiedenen Outcome-Maßen korreliert, um zu überprüfen, ob diejenigen Aspekte, die laut Therapietheorie für die Veränderungen im Patienten verantwortlich sein sollen, es auch in der Praxis sind. Hier zeigte sich, dass weder der psychodynamische noch der kognitiv-behaviorale Prototyp die Faktoren, die letztendlich bei den psychodynamisch behandelten Patienten mit PTSD zu den erwünschten Veränderungen geführt hatten, zu erfassen vermochte. In der anderen psychodynamisch behandelten Stichprobe zeigten sich positive Korrelationen des Outcomes sowohl mit dem psychodynamischen als auch mit dem kognitiv-behavioralen Prototyp. In der kognitiv-behavioral behandelten Patientengruppe hingegen zeigte sich ein positiver Zusammenhang zwischen einem Therapieerfolg und dem psychodynamischen Prototyp, nicht jedoch mit dem kognitiv-behavioralen Prototyp. Ablon und Jones (1998) interpretieren diese Ergebnisse als vorsichtigen Hinweis darauf, dass auch kognitiv-behaviorale Behandlungen einige typische psychodynamische Techniken enthalten sollten, weil gerade diese zu den Veränderungen im Patienten führen könnten.

Zum Vergleich einer manualisierten kognitiv-behavioralen und einer interpersonalen Kurzzeittherapie bei depressiven Patienten griffen Ablon und Jones (1999) auf das Datenmaterial aus dem TDCRP (vgl. 2.2 in diesem Kapitel) zurück. Hier wurden die Sitzungen vier und zwölf jeweils von mindestens zwei Beurteilern mit dem PQS eingeschätzt. 
Dabei fanden sich keine Unterschiede zwischen frühen und späten Sitzungen, sodass die PQS-Ratings für die weiteren Analysen über beide Sitzungen und alle Rater gemittelt werden konnten. Zwischen den beiden Therapieansätzen fanden sich bei 48 der 100 Items des PQS bedeutsame Unterschiede. Diese beziehen sich erneut (vgl. Jones \& Pulos, 1993) v.a. auf Merkmale der therapeutischen Interventionen und Haltungen. So sind die Therapeuten in der Interpersonalen im Vergleich zu denen in der kognitiv-behavioralen Therapie empathischer, sensibler und fokussieren interpersonale und romantische Beziehungen sowie Kindheitserinnerungen stärker. Kognitiv-behaviorale Therapeuten geben häufiger Hausaufgaben, besprechen v.a. kognitive Themen, steuern und kontrollieren die Interaktion stärker. Bei 52 der 100 Items gab es jedoch keine Differenzen zwischen den beiden untersuchten Therapieansätzen. Wiederum zeigen sich die fehlenden Unterschiede v.a. bei den Prozessmerkmalen, die die Haltung, das Verhalten oder den emotionalen Zustand des Patienten in der Therapie beschreiben (29 von 52 Items). Weiterhin überprüften Ablon und Jones (1999) in dieser Untersuchung auch, ob bestimmte Items des PQS den Therapieerfolg vorhersagen können. 23 von 100 Items waren dazu in der Lage. 22 dieser waren „Patientenitems“, wobei wiederum 18 davon sich nicht zwischen den beiden Therapieansätzen unterschieden hatten.

Ablon und Jones (2002) ließen jeweils die 4. und 12. Sitzung von 35 mit Interpersonaler und 29 mit kognitiv-behavioraler Therapie behandelten Patienten mit dem PQS beurteilen. Sie konnten zeigen, dass sich sowohl der IPT- und der KBT-Prototyp als auch die implementierte interpersonale und kognitiv-behaviorale Therapie sehr ähnlich sind. Die Autoren vermuten die Ursachen für diesen Befund einerseits im Patienten, der sich unabhängig vom Therapieansatz ähnlich verhält (vgl. Ablon \& Jones, 1999) und andererseits in der Art und Weise, psychologische Konstrukte und Prozesse zu beschreiben. Sie nehmen an, dass sich Aspekte, die sich zwar aufgrund unterschiedlicher theoretischer Hintergründe in ihrer Benennung stark unterscheiden, möglicherweise in der Umsetzung in der Therapie ähneln und dass diese Ähnlichkeit evtl. erst durch die Untersuchung des Therapieprozesses sichtbar wird.

Eine weitere Untersuchung des Therapieprozesses führten Ablon, Levy und Katzenstein (2006) bei 17 Patienten mit einer Panikstörung, die in einem naturalistischen Setting behandelt wurden, durch. Obwohl die behandelnden Psychotherapeuten ihre primäre theoretische Orientierung selbst als psychodynamisch bezeichneten, zeigte diese Studie, dass auch als kognitiv-behavioral apostrophierte Interventionen zur Anwendung kamen. Gerade hier wird die Bedeutung, die Therapieprozessforschung im Rahmen einer effektiven 
Behandlung einnimmt, deutlich. Die Bezeichnung eines bestimmten Therapieansatzes sagt demnach nicht unbedingt etwas über seine Implementierung in die Praxis aus (vgl. a. Caspar \& Jacobi, 2005). Orientierte sich die Therapie am interpersonalen oder am psychodynamischen Prototyp, zeigte sich ein positives Therapieoutcome. Dieser Zusammenhang zeigte sich jedoch nicht mit dem kognitiv-behavioralen Prototyp. 28 der 100 Items des PQS erwiesen sich als signifikante Prädiktoren des Therapieerfolgs; 11 davon beschreiben den emotionalen Zustand oder das Verhalten des Patienten.

Das PQS wurde für den deutschen Sprachraum von der Arbeitsgruppe um C. Albani übersetzt (Albani et al., 2000). Zur Übersetzung wurden unabhängig voneinander drei deutsche Versionen erarbeitet. Nach der gemeinsamen Diskussion dieser Übersetzungen gemeinsam mit E. Jones wurde eine endgültige Version im Konsens erstellt. Albani et al. (2000) untersuchten damit den Therapieprozess einer 517 Stunden dauernden psychoanalytischen Behandlung.

\subsection{Bewertung des PQS}

Ein möglicher Kritikpunkt am PQS (vgl. Watzke, 2002) ist das hier als Antwortmodus verwendete „erzwungene Verteilungsverfahren“. Dieses kann zu methodischen Artefakten führen, wenn die einzuschätzenden Prozessvariablen in einer bestimmten Therapiesitzung eben nicht normalverteilt auftreten, wie es beim forced Q-sort gefordert wird. Die Einschätzungen dürften deshalb eigentlich nur in Relation zu den Einschätzungen der anderen Items innerhalb derselben Analyseeinheit (Therapiestunde) miteinander verglichen werden. Dies kann immer dann zu Problemen führen, wenn über mehrere Analyseeinheiten die Einschätzung für ein Item gemittelt wird, um z.B. Vergleiche zwischen verschiedenen Therapieansätzen vorzunehmen. Hier sind Validitätseinschränkungen denkbar.

Obwohl Jones (z.B. Jones et al., 1988; Jones et al., 1991; Jones \& Pulos, 1993) betont, dass die Items des PQS theoriefrei konstruiert sind, sehen Albani und Blaser (2005) einige der Items als deutlich von bestimmten theoretischen Konzepten geprägt (z.B. Item Q36 „Der Therapeut weist auf Abwehrmechanismen hin“ und Q 38 ,Es werden spezifische Aktivitäten oder Aufgaben besprochen, die der Patient außerhalb der Sitzung in Angriff nehmen soll."). Auch Watzke (2002) weist darauf hin. Ihrer Ansicht nach seien einige der Items, die psychoanalytische Sachverhalte beinhalten, elaborierter (z.B. „Abwehrhaltung“ (Item 36), „abgewehrte oder unbewusste Wünsche“ (Item 67), „therapeutische Neutralität (Item 93)) als die, die verhaltenstherapeutische Konstrukte beschreiben. Zentrale, verhaltenstherapeutisch 
relevante Begriffe wie Störungsmodell, dysfunktionale Schemata, Verstärkung etc. würden demgegenüber gar nicht berücksichtigt. Insgesamt sollten solche „theoriebelasteten“ Items daher möglichst vollständig eliminiert werden.

Weiterhin problematisch am PQS ist die Tatsache, dass die Bedeutung der Kategorie „neutral“ nicht immer gleich ist. So bedeutet die Wahl dieser für ein Item manchmal, dass ihm in dieser Stunde keine Bedeutung zukommt. Manchmal jedoch sind die Kategorien auch als Kontinuum zu verstehen, so dass "neutral“ dann bedeutet, dass der Therapeut z.B. nur „ein paar oder nur unauffällige“ Beziehungsdeutungen gibt. Leider gibt es hier noch keine Untersuchungen zur differentiellen Reliabilität dieser Items.

Den genannten Schwächen des PQS stehen einige Vorteile gegenüber. So können auch praktisch relativ unerfahrene Personen das PQS anwenden. Bei solchen Beurteilern sind Verzerrungseffekte aufgrund impliziter Theorien (z.B. in Bezug auf das gemeinsame Auftreten bestimmter Interventionen) weniger wahrscheinlich. Die Konsequenz solcher Fehler wäre eine Überschätzung der Unterschiede von verschiedenen Therapieansätzen. Nachteil bei der Arbeit mit weniger erfahrenen Ratern ist, dass sie evtl. bestimmte komplexe Interventionen nicht erkennen, was zu einer Unterschätzung der Unterschiede führen würde. Werden jedoch dennoch Differenzen aufgedeckt, spricht dies in diesem Zusammenhang für ihre Richtigkeit.

Ein weiterer Aspekt, der für die Verwendung des PQS spricht, ist seine breite Anwendbarkeit auf ganz unterschiedliche Therapieansätze sowie die Möglichkeit, den Therapieprozess als ganzen, und nicht nur einige Aspekte oder zeitlich begrenzte Segmente erfassen zu können. Gerade weil jeder Therapieprozess aus einer interaktionellen Struktur besteht, ist es wichtig, ihn über einen längeren Zeitraum beobachten und dann danach beurteilen zu können. Anders als bei anderen Instrumenten werden mit dem PQS außerdem nicht nur therapeutische Interventionen oder Einstellungen und Verhaltensweisen des Patienten oder Patient-Therapeut-Interaktionen erfasst, sondern alle Aspekte gleichzeitig. So ist die Handhabung und Einarbeitung in das PQS zwar relativ komplex, allerdings können eben genau dadurch auch komplexere Informationen über den Therapieprozess ermittelt werden.

Aus dem deutschen Sprachraum gibt es bisher noch keine Studien zu Therapieprozessvergleichen von verschiedenen Therapieansätzen mit Hilfe des PQS. Im Rahmen der vorliegenden Arbeit wird ein solcher Vergleich angestrebt. 


\subsection{Erste allgemeine Befunde aus der Psychotherapieprozessforschung: Unterscheidung von unspezifischen und spezifischen Wirkfaktoren}

3.5.1 Theoretische Annahmen und empirische Befunde zu unspezifischen Wirkfaktoren

Psychotherapieprozessforschung untersucht u.a., welche Prozessfaktoren zu den angestrebten Veränderungen im Patienten führen („Wirkfaktoren“). Es herrscht jedoch wenig Einigkeit darüber, in welchem Ausmaß unspezifische und in welchem Ausmaß spezifische Faktoren für die Effekte von Therapien verantwortlich sind. Weinberger (1995) bemängelt außerdem das Fehlen einer einheitlichen Theorie „unspezifischer Wirkfaktoren“. Beutler (1995) erklärt dies damit, dass unspezifische Faktoren eben nicht in allen Therapien immer genau gleich seien und auch nicht unabhängig vom jeweiligen Therapeuten oder Patienten und von deren Interaktion immer gleich wirkten („Monosubstanz-Mythos“; Meyer, 1990b, S. 156). Stattdessen sollen sie sich in der Art, wie sie aus verschiedenen theoretischen Perspektiven heraus umgesetzt oder durch verschiedene Interventionen entwickelt werden, unterscheiden.

Ein erster Versuch, unspezifische Wirkfaktoren überhaupt zu definieren, wurde von J.D. Frank unternommen (z.B. 1961, 1973, 1982, 1992). Dieser ging davon aus, dass für psychotherapeutische Veränderungen unabhängig vom Therapieansatz folgende Aspekte relevant sind:

1. Aufbau und Aufrechterhaltung einer vertrauensvollen, emotionalen Patient-Therapeut-

Beziehung sowie Verständnis und Empathie für den Patienten.

2. Vermittlung eines Störungsmodells.

3. Gabe von Informationen über die Störung, ihr Zustandekommen, die möglichen

Veränderungen, über das Behandlungsrationale und das therapeutische Vorgehen.

4. Wiederaufbau bzw. Mobilisierung von Hoffnung und Sinn.

5. Erproben neuer Verhaltensweisen im geschützten Rahmen.

Ein weitaus komplexeres und vollständigeres Modell unspezifischer Wirkfaktoren legten Orlinsky und Howard mit ihrem „Generischen Modell der Psychotherapie“ (1987) vor. Die Basis dieses Modells bilden Einzelbefunde aus der Prozess-Outcome-Forschung. Es versucht, alle im Therapieprozess Einfluss nehmenden Aspekte, unabhängig vom jeweiligen Therapieansatz, zu integrieren. Orlinsky und seine Kollegen ergänzen das Modell auch heute noch immer wieder um die aktuellsten empirischen Befunde (vgl. Orlinsky et al., 2004). In diesem Modell wird davon ausgegangen, dass der individuelle (z.B. vergangene Erfahrungen des Patienten) sowie der kulturelle und soziale Kontext (z.B. stationäre oder ambulante 
Behandlung) den therapeutischen Prozess sowie das Outcome und den Output beeinflussen. Im Therapieprozess werden sechs verschiedene Facetten voneinander unterschieden: formale Aspekte (,therapeutischer Kontrakt“", z.B. Absprachen über Behandlungsziele und methoden), technische Aspekte (,therapeutische Operationen“: Anwendung des entsprechenden Fachwissens des Therapeuten), interpersonale Aspekte (,therapeutische Beziehung“ zwischen Patient und Therapeut), intrapersonale Aspekte (,,innere Selbstbezogenheit": Selbsterleben in Beziehungen der Beteiligten in ihrer jeweiligen Rolle), „In-session-Aspekte“ (unmittelbare Auswirkungen der Therapiesitzung) sowie zeitliche Aspekte („sequenziell verlaufender Prozess“: Ablauf einer Sitzung und Therapieverlauf insgesamt). Die genannten Aspekte sind funktional miteinander verbunden und definieren erst dadurch das psychotherapeutische System. In ihrer Übersicht der empirischen Befunde zu unspezifischen Wirkfaktoren (Orlinsky et al., 2004) zeigt sich ein positiver Zusammenhang mit dem Outcome, wenn der Patient seine zentralen Probleme kognitiv verarbeiten kann, wenn er sie dem Therapeuten verständlich schildern kann, und wenn der Patient - mit Unterstützung des Therapeuten - den Fokus auf diesen Problemen halten kann.

Therapeutische Interpretationen sowie eine positive Reaktion des Patienten auf therapeutische Interventionen korrelierten ebenfalls signifikant mit einem positiven Outcome (vgl. Orlinsky et al., 2004). Auch die therapeutische Beziehung (,,working alliance“; vgl. Greenson, 1967) übt, unabhängig vom Therapieansatz, einen bedeutsamen Einfluss auf das Therapieergebnis aus (vgl. Orlinsky et al., 2004). Unter ,alliance“ wird eine positive Zusammenarbeit zwischen Klient und Therapeut verstanden. Diese ermöglicht es dem Patienten überhaupt erst, die Behandlung zu akzeptieren und das in der Therapie Gelernte umzusetzen. Eine gute therapeutische Beziehung zeichnet sich u.a. durch ein hohes Maß an Zusammenhalt und beiderseitigem Engagement sowie durch die Glaubwürdigkeit des Therapeuten aus (vgl. Orlinsky et al., 2004). Die Wirkung einer solchen besteht wahrscheinlich darin, dass sie dem Patienten eine korrigierende emotionale Erfahrung vermittelt, es damit also zur Löschung negativer und zum Erwerb neuer interaktioneller Verhaltensweisen und zur kognitiven Umstrukturierung kommt (vgl. Laireiter, 2000). Martin, Garske und Davis (2000) berechneten in ihrer Meta-Analyse eine durchschnittliche gewichtete Korrelation von $r=.22$ für den Zusammenhang von Allianz und Therapieoutcome. Auch die Offenheit des Patienten spielt für ein positives Outcome eine große Rolle (vgl. Orlinksy et al., 2004), wobei diese jedoch immer im Zusammenhang mit der Passung des Patienten auf das Treatment, seiner Kooperation und seinem Beitrag zur therapeutischen Beziehung zu sehen ist. 
Theoretischer und empirischer Hintergrund

Psychotherapieprozessforschung

Insgesamt gibt es mittlerweile ganz verschiedene Annahmen darüber, welche unspezifischen Wirkfaktoren es überhaupt in einer Psychotherapie gibt und wie diese wirksam werden (vgl. Karasu, 1986; Grawe, 1995; Laireiter, 2000). Lambert und Ogles (2004) versuchten, alle bekannten unspezifischen Wirkfaktoren zu kategorisieren. Dazu ordneten sie diese jeweils einer von drei Kategorien zu, Unterstützung, Lernen und Handlung (vgl. Tab. 3). Sie gehen davon aus, dass die supportiven Funktionen einer Psychotherapie Änderungen in den Glaubenssätzen und Haltungen des Patienten vorbereiten, die zu Handlungen beim Patienten führen. Eingebettet in eine kooperative Arbeitsbeziehung zwischen Patient und Therapeut, die ein steigendes Sicherheits- und Vertrauensgefühl beim Patienten mit sich bringt, werden Problemkonzepte verändert. Therapeutische Techniken wie z.B. reframing oder das Durcharbeiten von Problemen sollen zu veränderten Handlungen und Haltungen führen.

Tabelle 3: Übersicht über unspezifische Therapieprozesse nach Lambert und Ogles (2004, S. 173).

\begin{tabular}{|l|l|l|}
\hline Unterstützung & Lernen & Handlung \\
\hline Katharsis & Ratschläge & Verhaltensregulation \\
\hline $\begin{array}{l}\text { Identifikation mit dem } \\
\text { Therapeuten }\end{array}$ & Affektive Erfahrungen & Kognitives Meistern \\
\hline Reduktion der Isolation & Angleichen & $\begin{array}{l}\text { Ermutigung dazu, sich seinen } \\
\text { Ängsten zu stellen }\end{array}$ \\
\hline Positive Beziehung & Kognitives Lernen & Risiken in kauf nehmen \\
\hline Rückversicherung & $\begin{array}{l}\text { Korrektive emotionale } \\
\text { Erfahrung }\end{array}$ & Anstrengungen meistern \\
\hline Nachlassen der Anspannung & Feedback & Modell \\
\hline Struktur & Einsicht & Praxis/Übung \\
\hline Therapeutische Allianz & Rational & Realitätsüberprüfung \\
\hline $\begin{array}{l}\text { Aktive Beteiligung des } \\
\text { Therapeuten/Patienten }\end{array}$ & $\begin{array}{l}\text { Exploration des inneren } \\
\text { Bezugsrahmens }\end{array}$ & Erfolgserlebnisse \\
\hline $\begin{array}{l}\text { Expertise/Erfahrung des } \\
\text { Therapeuten }\end{array}$ & $\begin{array}{l}\text { Veränderung der Erwartungen } \\
\text { bzgl. der eigenen Effektivität }\end{array}$ & Durcharbeiten \\
\hline $\begin{array}{l}\text { Therapeutische Wärme, } \\
\text { Respekt, Empathie, Akzeptanz } \\
\text { und Echtheit }\end{array}$ & & \\
\hline Vertrauen & & \\
\hline
\end{tabular}

\subsubsection{Vergleich des Einflusses von unspezifischen und spezifischen Wirkfaktoren}

Ahn und Wampold (2001) untersuchten in ihrer Meta-Analyse die spezifische Wirkung unterschiedlicher psychologischer Behandlungen. Dazu berücksichtigten sie alle zwischen 1990 und 1999 in renommierten Zeitschriften für Klinische Psychologie („Behaviour Research and Therapy“, „Behavior Therapy“, „Journal of Consulting and Clinical 
Psychology“, ,Journal of Counseling Psychology“) erschienenen Publikationen, die die Wirksamkeit einer bestimmten psychotherapeutischen Behandlung bei einer bestimmten Störung oder bei einem bestimmten Problem erforscht hatten. Auch mussten diese die relevanten Statistiken, die zur Berechnung einer Meta-Analyse notwendig sind, angegeben haben. Diese Studien waren sog „Komponenten-Studien“ (vgl. Ahn \& Wampold, 2001). Solche versuchen, wirksame Therapieelemente zu identifizieren, in dem sie Behandlungen mit und ohne diese Elemente miteinander vergleichen. Entweder werden dabei bestimmte potentiell wirksame therapeutische Elemente in einer der beiden Behandlungsgruppen hinzugefügt (konstruktive Strategie) oder entfernt (Dismantling-Strategie). Damit eine Studie in diese Meta-Analyse aufgenommen wurde, musste die psychotherapeutische Behandlung auf einem bereits etablierten Therapieansatz beruhen, die Behandlung musste in Form eines Manuals genau beschrieben sein, die wirksamen Elemente der Behandlung mussten in der Studie benannt worden sein und es musste ein Gruppenvergleich stattgefunden haben (mit Hilfe einer konstruktiven oder Dismantling-Strategie).

Insgesamt gingen 27 Behandlungsvergleiche aus 20 verschiedenen Studien in die MetaAnalyse ein. Es zeigte sich kein Beleg dafür, dass spezifische Wirkfaktoren für das positive Outcome der Behandlungen verantwortlich waren, da sich die über die 27 Vergleiche aggregierte Effektgröße nicht signifikant von Null unterschied. Dies bedeutet, dass hier v.a. unspezifische Faktoren ihre Wirkung entfalteten. Wampold (2001) zeigte außerdem, dass unspezifische im Vergleich zu spezifischen Faktoren neunmal mehr Varianz im Outcome aufklären können.

Tschuschke et al. (1994) hingegen sehen neben der Bedeutung unspezifischer auch die Bedeutung spezifischer Faktoren bestätigt. Sie führen an, dass Patienten in PlaceboTherapien $^{9}$ (also einer unspezifischen Psychotherapie z.B. in Form von stützenden Gesprächen) deutlichere Verbesserungen zeigen als Patienten in Warte- oder Kontrollgruppen (Effektstärken von ES $=.42$ bis ES = .58). Spezifische Psychotherapien hingegen zeigen eine noch deutlich höhere durchschnittliche Effektstärke von .85 (vgl. Smith et al., 1980); hier scheinen also weitere spezifische Faktoren zu wirken.

\footnotetext{
${ }^{9}$ Die Sinnhaftigkeit einer Placebo-Bedingung ist in Bezug auf die Psychotherapie allerdings durchaus in Frage zu stellen. Nach Meyer (1990a, S. 288, vgl. a. Reinecker, 2000, S. 58) werden psychosoziale Interventionen entweder als hilfreich betrachtet und sind dann kein Placebo, oder sie werden als unwirksam betrachtet und sind dann eben unwirksam.
} 
Nach den aufgeführten Befunden spielen demnach sowohl spezifische als auch unspezifische Wirkfaktoren bei therapeutischen Veränderungen eine Rolle. Möglicherweise kommt unspezifischen Wirkfaktoren jedoch die größere Bedeutung zu.

\subsection{Vergleich der unspezifischen und spezifischen Prozessmerkmale in psychodynamischen und kognitiv-behavioralen Therapien}

Im Hinblick auf die Zielstellung der vorliegenden Arbeit sollen nun Befunde zu unterschiedlichen und gemeinsamen Prozessfaktoren von psychodynamischen und kognitivbehavioralen Therapien dargestellt werden. Dieser Vergleich bezieht sich auf Unterschiede bzw. Gemeinsamkeiten hinsichtlich des Verhaltens und emotionalen Zustands des Patienten, auf Unterschiede bzw. Gemeinsamkeiten hinsichtlich therapeutischer Interventionen und Verhaltensweisen und auf Unterschiede bzw. Gemeinsamkeiten hinsichtlich der Interaktion zwischen Patient und Therapeut. Da es mittlerweile eine große Vielzahl an Prozess-Befunden in Bezug auf Gemeinsamkeiten und Unterschiede zwischen verschiedenen Therapieansätzen gibt, ist die folgende Aufzählung und die damit getroffene Auswahl von Studien exemplarisch $\mathrm{zu}$ verstehen.

Bei kognitiv-behavioralen im Vergleich zu psychodynamischen Therapien liegt der Fokus therapeutischer Interventionen auf der Veränderung von Kognitionen (Jones \& Pulos, 1993; Ablon \& Jones, 1998, 1999; Watzke, 2002) und Verhalten (Sloane et al., 1975; Watzke, 2002, Malik, Beutler, Alimohamed, Gallagher-Thompson \& Thompson, 2003). Auch werden die Zukunftsperspektiven des Patienten häufiger thematisiert (Goldfried, Castonguay, Hayes, Drozd \& Shapiro, 1997). In psychodynamischen Therapien hingegen stehen Emotionen (Sloane et al., 1975, Jones \& Pulos, 1993; Goldfried et al., 1997; Goldfried, Raue \& Castonguay, 1998; Ablon \& Jones, 1999; Malik et al., 2003) und vergangene Erfahrungen (Jones \& Pulos, 1993; Ablon \& Jones, 1998) stärker im Fokus der Aufmerksamkeit. Im Hinblick auf die Fokussierung der interpersonellen Ebene gibt es keine eindeutigen Befunde (Jones und Pulos, 1993; Silove, Parker \& Manicavasagar, 1990; Kerr, Goldfried, Hayes, Castonguay \& Goldsamt, 1992). Werden therapeutische Interventionen betrachtet, zeigen sich zwischen beiden Therapieansätzen keine Unterschiede hinsichtlich der „Interpretationen“ (Wiser \& Goldfried, 1996), der „Klarifikationen“ (Sloane et al., 1975) und der „Häufigkeit von Fragen“ (Sloane et al., 1975; Stiles, Shapiro \& Firth-Cozens, 1988). Jedoch sind kognitivbehavioral orientierte Therapeuten konfrontativer (Sloane et al., 1975; Jones \& Pulos, 1993; Ablon \& Jones, 1999), geben mehr Ratschläge (Sloane et al., 1975; Stiles et al., 1988) und 
vermitteln mehr Informationen (Sloane et al., 1975; Goldfried et al., 1997). Psychodynamisch orientierte Therapeuten hingegen wenden mehr „Reflexion“ als therapeutische Intervention an (Stiles et al., 1988; Wiser \& Goldfried, 1996). Das Empathielevel als Hinweis auf die therapeutische Haltung unterscheidet sich nicht zwischen den beiden Therapieansätzen (Sloane et al., 1975; Wiser \& Goldfried, 1996); Verhaltenstherapeuten im Vergleich zu psychodynamischen Therapeuten sind jedoch direktiver (Sloane et al., 1975; Brunink \& Schroeder, 1979; Malik et al., 2003), aktiver (Sloane et al., 1975), weniger zurückhaltend (Sloane et al., 1975; Stiles et al., 1988) und unterstützen ihre Patienten stärker emotional (Brunink \& Schroeder, 1979). Auch in der Interaktion zwischen Patient und Therapeut gibt es Unterschiede zwischen beiden Therapieansätzen. Psychodynamische Therapeuten explorieren häufiger Faktoren, die die Therapie evtl. stören könnten (Jones \& Pulos, 1993; Ablon \& Jones, 1998, 1999; Goldfried et al., 1998). Im Hinblick auf Verhaltensweisen des Patienten bzw. auf seinen emotionalen Zustand in der Therapie ließen sich keine Unterschiede zwischen diesen beiden Therapieansätzen finden (vgl. Jones \& Pulos, 1993; Ablon \& Jones, 1999).

Blagys und Hilsenroth (2002) schlussfolgern, dass kognitiv-behavioral orientierte Therapeuten die folgenden Techniken generell häufiger nutzen als psychodynamisch orientierte Therapeuten: a) Gabe von Hausaufgaben und Aktivitäten außerhalb der Therapiesitzungen, b) direkte Führung/Strukturierung innerhalb der Sitzungen, c) Vermitteln spezifischer Coping-Strategien, d) Fokus auf künftigen Erfahrungen des Patienten, e) Vermitteln von Informationen über die Behandlung, über die Störung oder über Symptome und f) Fokussierung kognitiver Erfahrungen.

\section{Die Generalisierte Angststörung}

\subsection{Das Störungsbild}

Die Generalisierte Angststörung (GAS) wird erst seit Erscheinen des DSM-III (APA, 1980) als eigenständiges Störungsbild der Achse I verstanden. Vorher wurde sie aufgrund ihrer heterogenen Diagnosekriterien als Prodromal- oder Residualsyndrom einer anderen Störung betrachtet (vgl. Kessler, Keller \& Wittchen, 2001).

Das Hauptmerkmal einer GAS sind anhaltende, übermäßige, als schwer kontrollierbar erlebte Ängste und Sorgen (,furchtsame Erwartung“, „,worry“) bzgl. mehrerer Ereignisse oder Tätigkeiten (APA, 1994), die nicht an spezifische Objekte oder bestimmte Situationen (wie

z.B. bei spezifischen Phobien) gekoppelt sind (,frei flottierende Ängste“, Rapee, 1991). Zwar 
unterscheiden sich die Inhalte dieser Sorgen nicht von den Sorgeninhalten psychisch unauffälliger Personen (Sorgenbereiche sind z.B. Finanzen, Krankheit und Gesundheit von Familienmitgliedern; vgl. Hiller, 2004), jedoch finden sich Unterschiede in formalen Aspekten wie Dauer, Häufigkeit und Intensität. Die Sorgendauer von Betroffenen ist viermal so lang wie die von gesunden Kontrollpersonen oder Patienten mit einer Sozialphobie (Hoyer, Becker \& Roth, 2001). 95 \% der Betroffenen geben an, sich auch schon bei vergleichsweise geringfügigen Anlässen zu sorgen (Hiller, 2004). Interpersonale Beziehungen sind oft ein Sorgenthema (Borkovec \& Costello, 1993; Borkovec, Ray \& Stöber, 1998; Hiller, 2004), was auch mit den gehäuft auftretenden unsicheren Bindungen zusammenhängen könnte (Borkovec et al., 1998). Nach den DSM-IV-Kriterien (APA, 1994) einer GAS muss das „Sich-Sorgen“ von körperlichen Symptomen wie Ruhelosigkeit, Konzentrationsschwierigkeiten, Reizbarkeit, Schlafproblemen, Muskelverspannungen, Herzrasen, Übelkeit, Schwindel oder leichter Ermüdbarkeit begleitet sein.

Die aktuellen Klassifikationssysteme DSM-IV (APA, 1994) und ICD-10 (Dilling, Mombour \& Schmidt, 1993) geben ähnliche Kriterien zur Diagnose einer GAS vor, allerdings räumt die ICD-10 anderen Störungen Priorität ein. Wenn also neben einer GAS-Symptomatik eine Panikstörung, phobische Störung, eine Zwangsstörung oder ein hypochondrische Störung vorliegt, ist laut ICD-10 die Diagnose einer GAS nicht zu vergeben (vgl. Dilling, Mombour, Schmidt \& Schulte-Markwort, 2004). Beide Klassifikationssysteme berücksichtigen jedoch nicht das für GAS-Patienten typische Vermeidungs- und Rückversicherungsverhalten (vgl. Zubrägel, Bär \& Linden, 2003). So rufen Betroffene häufig bei Familienmitgliedern an, um sich nach deren Wohlbefinden zu erkundigen. Werden sie an solchen „Kontrollanrufen“ gehindert, tritt Unruhe, Angst oder verstärktes Sich-Sorgen auf.

Differentialdiagnostisch ist die Generalisierte Angststörung von anderen Angststörungen und von depressiven Syndromen abzugrenzen. Im Vergleich zu Patienten mit anderen Angststörungen verbringen Patienten mit GAS deutlich mehr Zeit am Tag mit „Sich-Sorgen““ Auch können sie diese schwerer kontrollieren und erleben sie als emotional sehr belastend. Anhand der genannten Kriterien unterscheiden sich Patienten mit einer GAS nur wenig von Patienten mit einer Depression. Die grübelnden Gedanken letzterer beschäftigen sich jedoch v.a. mit Trauer, Verlust oder eigenem Versagen, während die Sorgen der Patienten mit GAS eher auf den Umgang mit künftigen Stressoren oder Bedrohungen vorbereiten sollen (Zubrägel et al., 2003). Die Sorgen im Rahmen einer GAS sind auch von den Zwangsgedanken einer Zwangsstörung zu unterscheiden. Diese erscheinen den Betroffenen 
als aufdringliche, ich-dystone Gedanken, die oft auch in Form eines Drangs oder eines Impulses auftreten. Die Sorgen bei einer GAS hingegen sind übertriebene, ich-syntone Sorgen über reale Alltagsprobleme.

Auf der Basis aktueller Klassifikationssysteme wie der ICD-10 (Dilling et al., 1993) und dem DSM-III und IV (APA, 1987,1994) wurden repräsentative Studien zur Epidemiologie der GAS durchgeführt. Diese zeigen eine Lebenszeitprävalenz der GAS zwischen 4 und 7 \% (vgl. Hoyer et al., 2003). Nach einer multizentrischen Studie der Weltgesundheitsorganisation WHO (Üstün \& Sartorius, 1995) liegt die Punktprävalenz in Deutschland mit 8,1 \% relativ hoch. Auch in der primärärztlichen Versorgung gehört die GAS mit bis zu 10 \% zu den häufigsten Angststörungen (Wittchen \& Hoyer, 2001). Die höchsten Prävalenzen zeigen sich im mittleren (Wittchen, Zhao, Kessler \& Eaton, 1994) bzw. höheren Alter (Carter, Wittchen, Pfister \& Kessler, 2001). Rogers et al. (1999) konnten einen erstmaligen Inzidenzanstieg zu Beginn des zweiten Lebensjahrzehnts und dann stabil hohe Inzidenzraten bis mind. zum 55. Lebensjahr finden. Trotz der hohen Prävalenz suchen die Betroffenen im Vergleich zu Patienten mit anderen Angststörungen am seltensten professionelle Hilfe auf. Zubrägel et al. (2003) vermuten den Grund dafür darin, dass die Patienten sich oft nicht bewusst sind, dass sie unter einer Angststörung leiden. Häufig fühlen sie sich „,einfach nur“ belastet oder überfordert. Leichsenring et al. (2002) merken jedoch an, dass Patienten mit einer GAS sich ebenso stark psychosozial beeinträchtigt fühlen wie depressive Patienten. Erst wenn weitere Störungen hinzukommen, suchen sie ärztlichen oder psychologischen Rat. Die GAS weist eine hohe Komorbidität mit anderen psychischen Störungen auf. Bei 16-59 \% treten komorbid Soziale Phobien, bei 21-55 \% einfache Phobien, bei 3-27 \% Panikstörungen, bei 8-39 \% Depressionen und bei 37-90 \% Persönlichkeitsstörungen auf, wobei die ängstlichvermeidende hier am häufigsten genannt wird (Zubrägel et al., 2003). Komorbiditäten üben einen negativen Effekt auf den Verlauf aus (Angst \& Vollrath, 1991). Achse-I-

Komorbiditäten korrelieren mit einem schlechteren Therapieoutcome (Durham, Allan \& Hackett, 1997). Wie bei anderen Angststörungen auch sind Frauen etwa doppelt so häufig betroffen wie Männer (Wittchen et al., 1994; Carter et al., 2001). Hoyer et al. (2003) geben aber zu bedenken, dass diese Befunde möglicherweise durch die höhere Komorbidität bei Frauen zustande kommen. So zeigten sich in der Studie von Maier et al. (2000) keine Geschlechts-Unterschiede. Etwa 80 \% der Patienten berichten von einem allmählichen, schleichenden Beginn, bei 85 \% kam es nicht zu Spontanremissionen (Noyes et al., 1992; Yonkers, Massion, Warshaw \& Keller, 1996). Noyes, Holt und Woodman (1996) zeigten, 
dass die GAS eine chronische Störung ist, die Schwankungen unterliegt, und unbehandelt meist ein Jahrzehnt oder länger anhält.

\subsection{Entstehung und Aufrechterhaltung einer Generalisierten Angststörung}

Die spezifischen Entstehungsbedingungen einer Generalisierten Angststörung sind bisher noch unklar. Diskutiert wird der Einfluss biologischer bzw. genetischer Faktoren (z.B. Noyes, Clarkson, Crowe, Yates \& McChesney, 1987; Kendler, Neale, Kessler, Heath \& Eaves, 1992) im Sinne einer biologischen Vulnerabilität für die Entwicklung pathologischer Angst (vgl. Nutt, 2001). Denkbar erscheint hier eine Störung der Neurotransmittersysteme (GABA, NA, 5-HT; vgl. Brawman-Mintzer \& Lydiard, 1997). Auch ein Einfluss kritischer Lebensereignisse wie z.B. traumatischer und belastender Ereignisse (Blazer, Hughes \& George, 1987; Roemer, Borkovec, Posa \& Lyonfields, 1991) und Stress („daily hassles“; vgl. Kendler, Karkowski \& Prescott, 1998; Baum \& Fleming, 1993; Regier et al., 1993) wird bei der Entstehung und Aufrechterhaltung der GAS vermutet. Aufgrund methodischer Mängel sind die Befunde aus den dazu bisher vorliegenden Studien jedoch nicht eindeutig interpretierbar (vgl. Nutt, 2001; Hiller, 2004).

Auch eine psychologisch bedingte Vulnerabilität wird diskutiert (vgl. Leibing et al., 2003), die u.a. im Erziehungsstil der Eltern (unsichere Bindung, Mangel an emotionaler Wärme, Überprotektion) gesehen wird. Ein solcher Erziehungsstil kann dazu führen, dass Kinder bedeutsame Umweltaspekte als unkontrollierbar und nicht zu bewältigen erleben. Dies kann zu Defiziten in der Bewältigungskompetenz führen und so die Entwicklung spezifischer Grundannahmen wie z.B. „Die Welt ist gefährlich“ bedingen (vgl. Leibing et al., 2003). Neben solchen Kognitionen spielen auch (unangemessene) Aufmerksamkeitsprozesse für die Genese und v.a. für die Aufrechterhaltung der Angst eine zentrale Rolle (vgl. Rapee, 1991; Barlow, 1988, 1991). Im Vergleich zu nichtängstlichen Kontrollpersonen nehmen Patienten mit Angststörungen bedrohliche Reize schneller wahr und interpretieren uneindeutige Reize als bedrohlicher („emotionaler Stroop“, „,negatives Priming“; Matthews, 1990). Die Sorgen bzw. ängstliche Erwartung von Betroffenen führen zu einer Verlagerung der Aufmerksamkeit auf anxiogene Kognitionen und so zu einem weiteren Erregungsanstieg. Diese Sorgen (,worry“), die als wenig bildhafte Gedankenketten ablaufen, sind funktional im Sinne eines Problemlöseprozesses (vgl. Borkovec, Hazlett-Stevens \& Diaz, 1999). Mögliche Bedrohungen werden vorweggenommen und die Suche nach einem Ausweg wird eingeleitet. Durch die Sorgen über die Verhinderung noch bedrohlicherer bildhafter Vorstellungen kommt 
es zu einer kurzfristigen Reduktion der physiologischen Erregung und der Angst (negative Verstärkung). Die Sorgen werden von den Patienten aber nicht bis zur befürchteten Katastrophe gedacht, sondern an einem Angst erzeugenden Punkt abgebrochen. Echte Problemlösungsansätze werden nicht „,durchdacht“, stattdessen beginnt das „Sich-Sorgen“ über dieses Thema wieder von neuem oder es wird zu einem anderen Sorgenthema übergegangen (vgl. Leichsenring et al., 2002). Sorgen stellen in diesem Modell eine Form der kognitiven Vermeidung dar, die zur Aufrechterhaltung der Angst beiträgt (Borkovec et al., 1999). Die emotionale Verarbeitung angstbesetzter Themen wird dadurch dauerhaft verhindert, eine Habituation kann nicht stattfinden (vgl. Linden, Bär, Zubrägel, Ahrens \& Schlattmann, 2002).

Die Aufrechterhaltung von ,worrying“ lässt sich auch durch das so genannte „,abergläubische Verstärkungsparadigma“ erklären. Da die in Sorgenketten enthaltenen Konsequenzen meist nicht eintreten, gehen die Patienten von einem Zusammenhang (im Sinne von Kontingenzen) zwischen dem „Sich-Sorgen“ und dem „Nicht-Eintreten“ der Befürchtungen aus (vgl. Zubrägel et al., 2003; vgl. Abb. 1).

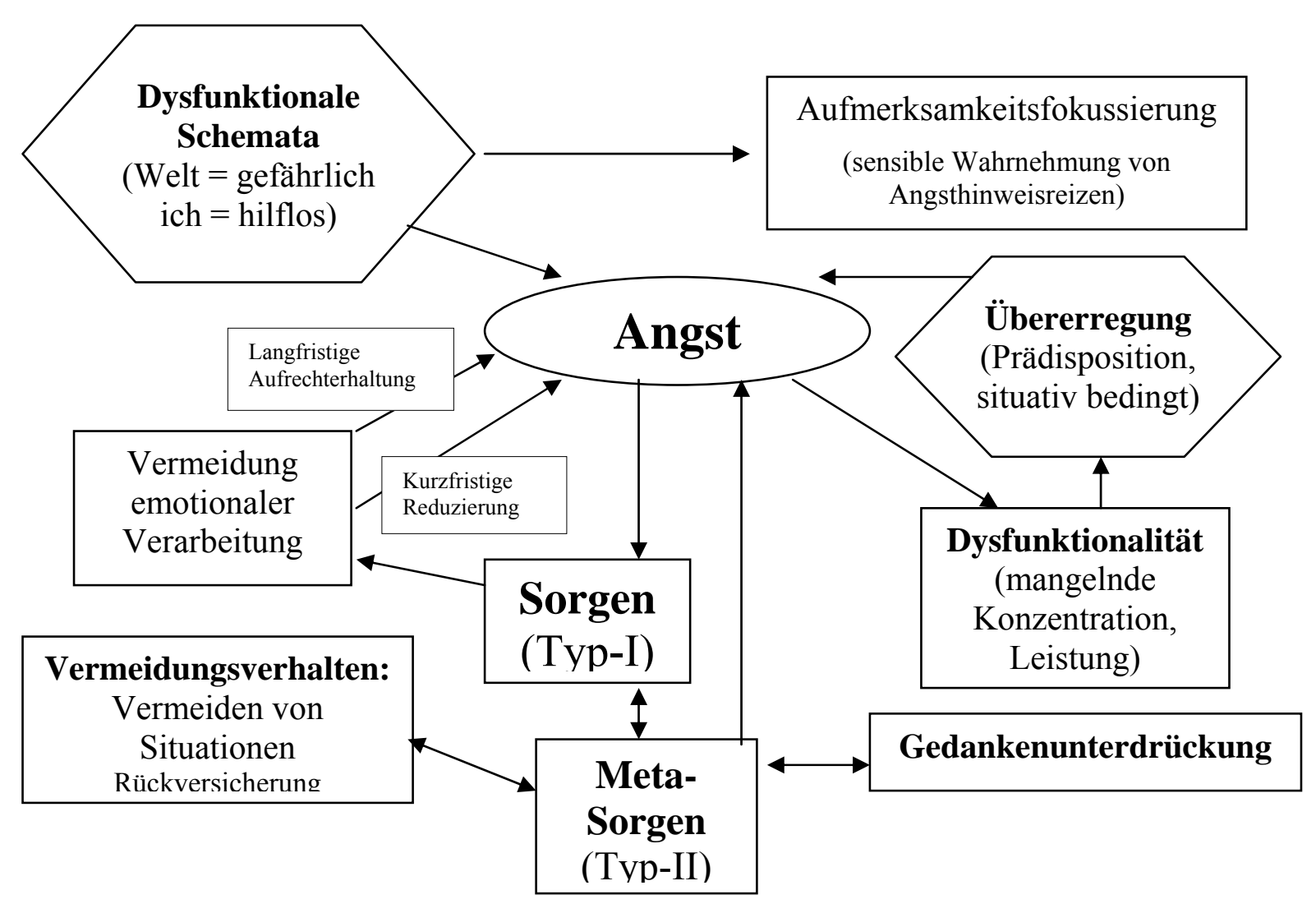

Abbildung 1: Vereinfachtes Störungsmodell der Generalisierten Angststörung aus verhaltenstherapeutischer Sicht (nach Leichsenring et al., 2002). 
Wells $(1997,1999)$ stellte die Bedeutung des Konzepts der Meta-Kognitionen bzw. TypII-Sorgen (vgl. Abb. 1) für ein verhaltenstherapeutisches Störungsmodell der GAS heraus. Typ-II-Sorgen beziehen sich im Gegensatz zu den Alltagssorgen (Typ-I-Sorgen) auf MetaKognitionen über die Sorgen vom Typ I (,Diese Sorgen machen mich noch verrückt“). Das „Sich-Sorgen“ über den Prozess des „Sich-Sorgens“ erleben die Betroffenen als unkontrollierbar; so wirkt es selbstverstärkend. Typ-II-Sorgen selbst lösen wiederum Angst, Vermeidungsverhalten und ineffektive Versuche aus, die Gedanken zu unterdrücken (,Sorgen helfen mir, mich vor unangenehmen Überraschungen zu schützen.“"), wodurch die Angst im Sinne eines Teufelskreisprozesses langfristig aufrechterhalten wird (vgl. Leibing et al., 2003; Barlow, 1988, 1991).

Empirische Befunde zeigten, dass Patienten mit GAS häufig unsichere Bindungen (CritsChristoph, Wolf-Palcio, Fichter \& Rudnick, 1995) und Traumatisierungen (Borkovec, 1994) erlebt hatten. Daraus leiteten Crits-Christoph et al. (1995) ein psychodynamisches Modell zur Erklärung der Entstehung einer GAS ab. Traumatisierungen des Patienten, die in jeder Lebensphase auftreten können, führen demnach zu bestimmten grundlegenden Schemata darüber, welche Fähigkeiten zum Erfüllen der eigenen Bedürfnisse zur Verfügung stehen. Solche Annahmen können z.B. die Frage, wie sicher es ist, von anderen Liebe, Schutz, Sicherheit oder Stabilität zu erhalten, betreffen. Diese Schemata verursachen starke Angst, weswegen sich der Patient stattdessen über weniger ängstigende Aspekte des Alltags sorgt (,worrying“). Diese für GAS-Patienten typischen furchtsamen Erwartungen oder Sorgen beziehen sich v.a. auf interpersonelle Interaktionen (Borkovec, Robinson, Pruzinsky \& DePree, 1983). In diesem Sinne haben Sorgen eine Abwehrfunktion; sie verhindern, dass sich die Patienten über noch emotionalere und für sie schlimmere Aspekte sorgen (CritsChristoph, Connolly, Azarian, Crits-Christoph \& Shappell, 1996). Die eigentlich gefürchteten Inhalte bleiben aber (un/)bewusst bestehen und können so zu wiederkehrenden maladaptiven Beziehungsmustern führen (,Zentrale Beziehungskonflikte“; vgl. Luborsky, 1995), durch die die gefürchteten Umstände manchmal überhaupt erst eintreten (z.B. einen geliebten Menschen zu verlieren). Crits-Christoph et al. (1995) gehen nicht davon aus, dass es für GAS-Patienten spezifische zentrale Beziehungskonflikte gibt, vielmehr sollen sich diese in Abhängigkeit von der individuellen Lebensgeschichte und deren Bewältigung entwickeln. Allerdings sollen in diesen Konflikten häufig ,primitive“, frühkindliche Wünsche auftreten (Crits-Christoph et al., 1995). Solche Zentralen Beziehungskonflikte stehen in der in dieser Studie implementierten 
psychodynamischen Therapie der GAS im Fokus der Aufmerksamkeit (vgl. 4.3.2 in diesem Kapitel).

\subsection{Therapie der Generalisierten Angststörung}

\subsubsection{Psychopharmako-Therapie}

Rund $50 \%$ der Patienten mit einer GAS werden heute psychopharmakologisch mit Benzodiazepinen (z.B. Lorazepam, Alprazolam und Buspiron) behandelt (Wittchen et al., 1994). Die anxiolytische Wirkung dieser Medikamente in der Akut-Behandlung ist erwiesen; positiv hervorzuheben sind die schnelle Wirksamkeit sowie die einfache und ökonomische Anwendung (Woods, Katz \& Winger, 1992). Dem stehen jedoch einige Nachteile gegenüber (vgl. Leichsenring et al., 2002):

1. Benzodiazepine bewirken keine anhaltende Besserung der Symptomatik: Bei Absetzung stellen sich die Ängste schnell wieder ein. Außerdem erwies sich ihr Einfluss auf die Kernsymptomatik, d.h. auf die Sorgen, als gering (Crits-Christoph et al., 1995).

2. Sie zeigen unerwünschte Wirkungen wie Schläfrigkeit, Depression, aggressives Verhalten, Beeinträchtigungen kognitiver (z.B. Gedächtnisdefizite) und psychomotorischer Funktionen (z.B. Koordinationsstörungen).

3. Bei längerer Einnahme kann es zu einer körperlichen Abhängigkeit und damit auch zu einem möglichen Missbrauch kommen.

4. Es zeigen sich unter der Behandlung hohe Rückfallraten von 60-80\% nach einem Jahr.

5. Es wurden negative Effekte auf die Entwicklung von Coping-Fähigkeiten gefunden.

6. Eine Subgruppe von Patienten (30\%) spricht überhaupt nicht auf die Medikamente an. Behandlungsversuche mit Beta-Blockern konnten bisher bestenfalls geringfügige Besserungen erzielen (Meibach, Mullane \& Binstok, 1987). Neuere Behandlungsansätze schließen die Gabe von Antidepressiva (trizyklische wie Imipramin, Amitryptilin und Doxepin und SSRIs) ein, da sich diese auch auf komorbide Störungen positiv auswirken können (Ballenger et al., 2001; Davidson, 2001).

\subsubsection{Psychotherapie}

Ruhmland und Margraf (2001) untersuchten metaanalytisch die Effekte von unterschiedlichen psychologischen Behandlungsarten bei erwachsenen Patienten mit GAS. Dazu wurden insgesamt 14 deutsch- oder englischsprachige Wirksamkeitsstudien psychotherapeutischer Behandlungen der GAS aus den Jahren zwischen 1966 und 1997 
berücksichtigt. Angewandte Entspannung zeigte die größte Effektstärke (ES = 1,65 in 2 Studien), es folgten die kognitiv-behaviorale ( $E S=1,43$ in 13 Studien), die Kognitive (ES = 1,20 in 6 Studien) und die psychodynamische Therapie (ES =1,13 in 2 Studien). Die Effekte der beiden erstgenannten Therapieansätze blieben auch sechs Monate nach Ende der Therapie noch erhalten, die der psychodynamischen Therapie hingegen nicht ( $E S=0,29$ in 1 Studie). Dieser mittlere Wert für die Effektstärke der psychodynamischen Therapie stammt aus zwei unterschiedlichen Behandlungsgruppen derselben Studie (Durham et al., 1994), die sich nur in der Behandlungsfrequenz unterschieden. Die Studie weist jedoch beträchtliche methodische Mängel auf: kein Therapiemanual, keine gesicherte Therapieintegrität, keine Angaben zum Konzept, nach dem die analytisch orientierte Kurzzeittherapie durchgeführt wurde sowie Unklarheiten, ob die durchgeführte Therapie für die Patientenstichprobe tatsächlich indiziert war (vgl. Leichsenring et al., 2002). Hier bedarf es demnach dringend weiterer Therapiestudien, die die Effektivität der psychodynamischen Therapie bei GAS weiter untersuchen. Biofeedback und systematische Desensibilisierung zeigten im Vergleich zu den bereits genannten Therapien bei Therapieende deutlich geringere Effektstärken von ungefähr $\mathrm{ES}=.80$.

\section{Kognitiv-Behaviorale Therapie}

Patienten mit GAS können die mit der Angst korrelierte zunehmende körperliche Anspannung nicht hinreichend früh und genau genug wahrnehmen und damit keine adäquaten Bewältigungsstrategien einsetzen. Deshalb lernen sie bei der Angewandten Entspannung zunächst, einen solchen beginnenden erhöhten Angst- und Aktivierungszustand wahrzunehmen (vgl. Öst, 1987). Sie lernen dann, sich in einem solchen Zustand zu entspannen und zwar bevor die Angst auf ihrem Höhepunkt ist („,coping“). Es werden in vivo Expositionen mit ängstigenden Situationen geübt. Vorteil dieser Methode ist ihre kurze Dauer. Öst und Breitholz (2000) fanden, dass die positiven Therapieeffekte auch nach einem Jahr noch stabil blieben. Allerdings scheint die Akzeptanz des Verfahrens relativ niedrig, denn immerhin $25 \%$ der Patienten brechen die Therapie ab (Ruhmland \& Margraf, 2001).

In der Angstmanagement-Therapie nach Suinn und Richardson (1971) werden beim Patienten gezielt Angst auslösende Situationen erzeugt. Der Patient soll nun lernen, mit der Angst unvereinbare Reaktionen, wie z.B. Entspannung, einzusetzen. Es wird keine Angsthierarchie erstellt, stattdessen stellt der Patient sich eine Angst auslösende Situation der Vergangenheit vor. (Körperliche) Angstreaktionen werden hier als diskriminative Reize 
verstanden, auf die der Patient so konditioniert wird, dass er seine Angst schon in einer frühen Phase zurückweisen kann.

In den kognitiven Theorien zur Entstehung und Aufrechterhaltung einer GAS haben furchtsame und dysfunktionale Kognitionen (z.B. Sorge) eine zentrale Bedeutung. Es wird angenommen, dass diese der Angstsymptomatik zugrunde liegen und sie die Störung aufrechterhalten. Deshalb werden in der Therapie zunächst solche automatischen, dysfunktionalen und irrationalen Gedanken identifiziert, und dann in funktionalere Gedanken umgearbeitet (Beck, 1999). Das Manual von Wells und Carter (1999) schlägt Verhaltensexperimente zur Umstrukturierung von Metakognitionen und zum Abbau der Gedankenunterdrückung vor, da davon ausgegangen wird, dass der Sorgenprozess von Angstpatienten v.a. durch ihre Meta-Sorgen aufrechterhalten wird (vgl. Abb. 1).

Kognitiv-behaviorale Therapien (vgl. Turowsky \& Barlow, 1996), bei denen kognitive und konfrontative Elemente mit Entspannungsverfahren (z.B. PMR nach Jacobson, 2002) kombiniert werden, sind von der American Psychological Association (APA) als empirisch validierte Therapien bei GAS anerkannt (Hahlweg, 1995; Chambless \& Ollendick, 2001). Eine niedrige Dropout-Rate von 8 \% (Ruhmland \& Margraf, 2001) zeigt, dass dieser psychotherapeutische Ansatz von den Patienten gut angenommen wird. Allerdings weisen Ruhmland und Margraf (2001) in ihrer Meta-Analyse auch darauf hin, dass ein großer Teil der Patienten mit GAS auch nach der psychologischen Behandlung noch relevante Symptome zeigt (Barlow, Rapee \& Brown, 1992; Borkovec \& Whisman, 1996; Fisher \& Durham, 1999), wenngleich die Symptome insgesamt durch die Therapie deutlich reduziert werden können. Die Effektivität der Behandlung muss demnach weiter optimiert werden. Deswegen werden in neueren Ansätzen (z.B. Turowsky \& Barlow, 1996; Zubrägel \& Linden, 2000; Leibing et al., 2003) der Prozess des Sich-Sorgens und die katastrophisierenden Gedanken, die zur Aufrechterhaltung der Sorgen führen, noch stärker fokussiert. Eine Sorgenexposition steht hier im Mittelpunkt der Behandlung. Diesen neueren Therapieansätzen (z.B. Leibing et al., 2003) sind die folgenden Therapieziele gemeinsam (z.B. Zubrägel \& Linden, 2000): Reduktion der vegetativen Erregung, Abbau der kognitiven Verzerrungen und Veränderung der Grundannahmen, Abbau des „Sich-Sorgens“, Abbau des Vermeidungs- und Rückversicherungsverhaltens sowie Verbesserung der Problem- und Stressbewältigung. Eine erste Untersuchung zur Effektivität eines solchen Programms (Linden, Zubrägel, Bär, Franke \& Schlattmann, 2005) deutet auf positive Effekte hin. Im Vergleich mit einer Wartegruppe erwies sich die kognitiv-behaviorale Behandlung deutlich überlegen, wobei sich die 
Veränderung auch als klinisch relevant herausstellte. Dieser Effekt blieb auch acht Monate nach Therapieende noch bestehen.

Das kognitiv-behaviorale Behandlungsmanual zur Therapie der Generalisierten Angststörung nach Leibing et al. (2003)

Auch der kognitiv-behaviorale Ansatz von Leibing et al. (2003) ist diesen neueren Ansätzen zuzuordnen. Diese Therapie, mit der die im Rahmen der vorliegenden Studie behandelten Patienten mit GAS therapiert wurden, ist auf 25(+5) Sitzungen ausgelegt, kann jedoch im Einzelfall auch verkürzt werden, wenn die festgelegten Therapieziele bereits vorher erreicht wurden. Das Manual wurde für die Behandlung von Patienten mit einer Generalisierten Angststörung ohne weitere Komorbiditäten entwickelt. Liegen weitere komorbide Störungen vor (wie z.B. eine andere Angststörung, eine Persönlichkeitsstörung oder eine mittelgradige depressive Episode), ist die Therapie inhaltlich und zeitlich an die jeweils spezifischen Bedingungen anzupassen. In den probatorischen Sitzungen (Sitzungen 15) werden v.a. die für die Therapie relevanten Informationen gesammelt und ein plausibles Störungsmodell zur Verdeutlichung der Zusammenhänge zwischen Gedanken, Gefühlen, körperlichen Veränderungen und Verhalten wird erarbeitet. Unter Einbezug erster therapeutischer Interventionen soll die Therapiemotivation gefördert und so eine gute Therapeut-Patient-Beziehung aufgebaut werden. In der 6.- 8. Sitzung werden erste Begriffe aus der kognitiven Therapie eingeführt (z.B. ,automatische Gedanken“, „Meta-Sorgen“ und „Denkfehler“). Der Patient wird dazu angeleitet, diese mit Hilfe von Rollenspielen oder Imaginieren von problematischen Situationen zu identifizieren. Auch lernt er in dieser Therapiephase ein Entspannungsverfahren mit dem Ziel, die erhöhte Anspannung und die vegetative Übererregbarkeit zu verringern. Ein zentraler Bestandteil in der mittleren Behandlungsphase (9.-13. Sitzung) ist die Reduktion der Sorgen. Dies soll über eine graduelle Exposition in sensu mit den für den Patienten typischen Sorgen geschehen. Gedankliche Vermeidung wird verhindert. Stattdessen sollen bildliche und nicht kognitive Inhalte evoziert und Sorgen sowie damit verbundene schlimme Befürchtungen konkretisiert werden. Es wird ein bildhaftes Katastrophen-Szenario unter Berücksichtigung aller Sinnesmodalitäten entwickelt, in dem der Patient für ca. 25 Minuten verbleibt. Patient und Therapeut beschreiben dieses Szenario immer wieder ganz genau, um eine gedankliche Vermeidung dieses durch den Patienten zu verhindern. In der 14. bis 18. Sitzung werden kognitive Verzerrungen und dysfunktionale Kognitionen abgebaut. Diese „kognitive Umstrukturierung“ lehnt sich an Techniken von Beck, Emery und Greenberg an (1985; z.B. Selbstbeobachtung, 
Reframing, Sokratischer Dialog, Zweispaltentechnik, Entkatastrophisieren, Realitätsprüfung, Alternativgedanken). Ziel ist, dass der Patient seine einseitige Aufmerksamkeitsausrichtung auf Gefahren reduziert. Das für GAS-Patienten typische ritualisierte Sorgen-Verhalten (Vermeidungs- und Rückversicherungsverhalten) wird in der 19.- 23. Sitzung im Sinne einer Exposition mit Reaktionsverhinderung unterbunden (z.B. sollen die Patienten es unterlassen, aus Sorge bei ihren Familienangehörigen anzurufen); neue Coping-Strategien sollen erworben und angstinkompatible Aktivitäten aufgebaut werden (z.B. über Problemlösetrainings, Übungen zur Selbstsicherheit, zum „Time Management“ und zur Abgrenzung von anderen; vgl. Brown, O'Leary \& Barlow, 1993). In der 24.-28. Sitzung werden die individuell bedeutsamen Behandlungskomponenten wiederholt und verfestigt; in der 29. und 30. Sitzung geht es um Rückfallprophylaxe und das Behandlungsende.

\section{Psychodynamische Therapie von Angststörungen}

Auch innerhalb der psychodynamischen Therapie richten sich die Bemühungen in der Behandlung der GAS auf die Berücksichtigung des Vermeidungsverhaltens, das bei vielen Angststörungen im Vordergrund steht und daher möglichst früh und aktiv in der Therapie zu bearbeiten ist (vgl. Bassler, 2004). Deshalb stehen gerade zu Beginn einer psychodynamischen Therapie verhaltenstherapeutische Techniken zur aktiven Angstbewältigung im Vordergrund. Meist können erst danach die eigentlichen Angst auslösenden unbewussten Konflikte aufgedeckt werden.

Insgesamt liegen in der psychodynamischen Psychotherapie nur wenig spezifische Therapiemanuale für Angststörungen vor (z.B. Milrod, Busch, Cooper \& Shapiro, 1997). Eine wichtige Grundlage für die Entwicklung des in der vorliegenden Studie verwendeten psychodynamischen Therapiemanuals zur Behandlung der GAS (Leichsenring, Winkelbach \& Leibing, 2005) bildet die supportiv-expressive Therapie nach Luborsky (1995).

In der supportiv-expressiven Therapie (SET, Luborsky, 1995) wird davon ausgegangen, dass die Ursachen psychischer Symptome in intrapsychischen und interpersonellen Konflikten (,Zentrale Beziehungskonflikte“) liegen. Diese werden als „Zentrales Beziehungskonfliktthema“ (ZBKT) formuliert, das immer drei Komponenten umfasst: einen Wunsch, eine Reaktion der Objekte auf diesen Wunsch und eine Reaktion des Selbst auf die Reaktion der Objekte. Die (generalisierte) Angst bei der GAS wird als Selbstkomponente verstanden. In der SET werden ZBKT-Muster mit dem Ziel fokussiert, das den Symptomen des Patienten zugrunde liegende ZBKT durchzuarbeiten und zu verstehen. Nach einer erfolgreichen Therapie ist das ZBKT zwar noch immer vorhanden, der Patient hat aber 
bessere Möglichkeiten entwickelt, wie er mit den eigenen Wünschen und den Reaktionen der anderen umgehen kann (Luborsky, 1995). Es wird zwischen expressiven (deutenden) und supportiven (stützenden, auch entwicklungsfördernden) Interventionen unterschieden (vgl. 1.2.2). Expressive Interventionen setzen eine unterstützende Beziehung voraus. Schwer gestörte Patienten, Patienten in einer akuten Krisensituation oder auch Patienten mit einer ichstrukturellen Schwäche sollten zunächst mit Hilfe supportiver Interventionen behandelt werden. Wichtig für diese ist die Herstellung einer ,hilfreichen Beziehung“ (Luborsky, 1976; vg. 1.2.4). Bei den supportiven Interventionsprinzipien vom Typ I vermittelt der Therapeut dem Patienten durch seine Sprache und sein Verhalten Verständnis, Akzeptanz, Sympathie und Wertschätzung, wodurch dieser sich beim Erreichen seiner Therapieziele unterstützt fühlen soll. Der Therapeut verstärkt den Patienten verbal immer dann, wenn dieser seinen Zielen ein Stück näher gekommen ist. Supportive Interventionen vom Typ II sind gleichbedeutend mit der Förderung eines „Wir-Bündnisses“ durch den Therapeuten. Auch soll der Patient dafür gelobt werden, dass er nun schon selbständig therapeutische Instrumente für sich nutzen kann, z.B. die verbesserte Selbstbeobachtung.

$\mathrm{Zu}$ den expressiven Techniken gehört das Finden des Zentralen Beziehungskonfliktthemas durch den Prozess des Zuhörens, Verstehens, Intervenierens und erneuten Zuhörens. Hier soll der Therapeut gleichschwebend aufmerksam sein, Vorannahmen und Hypothesen unberücksichtigt lassen und sich so für neue Erfahrungen öffnen. Nach der Phase des Zuhörens reflektiert und beurteilt er das Gehörte und bildet Hypothesen über das ZBKT, die Symptomatik und ihren Zusammenhang. Um das ZBKT verstehen zu können, sollte der Therapeut wissen, welche (Angst-) Symptome der Patient hat und wie diese mit dessen Beziehungsproblemen zusammen hängen. Dazu gehört zu klären, welche durchgehenden Wünsche, Reaktionen der Objekte und Reaktionen des Selbst sich in den Erzählungen von Beziehungsepisoden des Patienten finden lassen. Um ein abgerundetes Bild eines ZBKT zu erhalten, sollte der Therapeut auf dessen Erscheinen in der aktuellen Beziehung zum Therapeuten, in den aktuellen Beziehungen außerhalb der Therapie und in früheren Beziehungen (v.a. zu den Eltern) achten. Die Symptomatik ist als eine Art Problemlösung des Patienten zu verstehen. Nachdem der Therapeut das ZBKT verstanden hat, richtet er seine Interventionen immer wieder auf eine oder mehrere Komponenten des ZBKTs aus. Auch stellt er gelegentlich einen Bezug zur Symptomatik her. Wenn er ein ZBKT deutet, sollte dieses dem Patienten möglichst bewusstseinsnah sein. Auch sollte der Therapeut den Patienten beim Verstehen seiner Beziehungsproblematik unterstützen, indem er z.B. 
Zusammenhänge zwischen verschiedenen Beziehungsproblemen verdeutlicht. Es werden dabei sowohl der Anteil des Patienten als auch der Anteil anderer sowie die Entstehung des ZBKTs und der primäre und sekundäre Krankheitsgewinn berücksichtigt. Das ZBKT sollte auch in der Beziehung zum Therapeuten besprochen werden (Übertragung, Gegenübertragung). Eine Deutung durch den Therapeuten findet erst statt, wenn dieser es verstanden hat. Dadurch lernt auch der Patient, sorgsam nach der Bedeutung des ZBKTs zu suchen. Im Laufe der Therapie begreift der Patient seine Symptome als Versuch, seinen Zentralen Beziehungskonflikt zu lösen.

Da bei der GAS wie beschrieben interpersonelle Aspekte eine große Rolle spielen (vgl. Borkovec et al., 1983), scheint die SET besonders geeignet zu ihrer Behandlung. CritsChristoph et al. (1995) haben die SET für die Behandlung der GAS modifiziert. Aus den typischerweise bei Patienten mit GAS zu findenden Eigenschaften und Erfahrungen (,worrying“, unsichere Bindungen, Traumatisierungen) leiten sie als spezifische Therapieelemente den Aufbau einer sicheren, hilfreichen Beziehung (als korrigierende emotionale Erfahrung) sowie die Ermutigung zum Ausprobieren neuer Verhaltensweisen und zum Aufgeben des Vermeidungsverhaltens ab. Auch werden für GAS-Patienten typische primitive Wünsche, Widerstände und Abwehrhaltungen sowie vergangene traumatische Erfahrungen besonders berücksichtigt.

Erste Hinweise auf die Effektivität dieses Verfahrens fanden Crits-Christoph et al. (1996) selbst. Hochsignifikante Prä-Post-Effekte zeigten sich hinsichtlich des ,,worrying“, der Angstsymptome, der Depressivität sowie im Hinblick auf interpersonale Probleme. Auch gab es keine Therapieabbrüche. Kritisch anzumerken ist, dass in dieser Studie nur 26 Patienten behandelt wurden und es keine Kontrollgruppe gab.

\section{Psychoanalytisch-orientierte Fokaltherapie der Generalisierten Angststörung nach}

Leichsenring et al. (2005)

Die psychodynamische Therapie der GAS, so wie sie in der vorliegenden Studie durchgeführt wurde, basiert auf dem Therapieansatz von Crits-Christoph et al. (1995), wurde aber modifiziert und von 12 auf $25(+5)$ Sitzungen erweitert.

In den probatorischen Sitzungen werden zunächst die für die Angstsymptomatik relevanten diagnostischen Informationen erhoben und das Zentrale Beziehungskonfliktthema formuliert. Unmittelbar vor Therapiebeginn wird ein Paktgespräch geführt, in dem grundlegende Vereinbarungen (wie Termine, Frequenz und Dauer der Sitzungen), das weitere Vorgehen sowie erste vorläufige Therapieziele festgelegt werden. Dies ist die Grundlage für 
die Zusammenarbeit von Patient und Therapeut sowie für die Etablierung einer hilfreichen Therapie-Beziehung. Die individuellen Therapieziele sollten immer einen Zusammenhang mit dem ZBKT aufweisen, sie sollten fokalen Charakter haben, in der Sprache des Patienten formuliert und in der Kurzzeittherapie erreichbar sein. Bereits in der Anfangsphase der Therapie (1.-13. Sitzung) fokussiert der Therapeut auf das ZBKT und versucht, einen Zusammenhang mit der Symptomatik der GAS herzustellen. Er untersucht das ZBKT in den aktuellen, in der Therapie- und in früheren Beziehungen. In der 13. Sitzung wird eine Zwischenbilanz gezogen, in der überprüft wird, inwieweit die gesteckten Ziele bisher erreicht werden konnten. Sind sie vollständig erreicht, kann die Therapie jederzeit (auch vor der 25. Sitzung) abgeschlossen werden. In der mittleren Phase der Therapie (Sitzungen 9-17) wird das ZBKT weiter verfeinert. Der Therapeut zeigt Zusammenhänge zwischen diesem und früheren Beziehungserfahrungen sowie mit der Therapiebeziehung auf. Falls Traumata in der Vorgeschichte existieren, werden diese meist in der mittleren Phase der Therapie vom Patienten offenbart. Gemeinsam werden sie empathisch behandelt. Der Fokus liegt aber nicht auf dem Trauma selbst, sondern darauf, wie es das ZBKT beeinflusst hat. In der Abschlussphase können die Symptome aufgrund des baldigen imaginierten Verlusts des Therapeuten und der Nichterfüllung des ZBKT-Wunsches (z.B. nach Sicherheit, Nähe, Liebe) wieder zunehmen. Deshalb wird als wichtig erachtet, dass der Therapeut den Patienten rechtzeitig auf das Therapieende vorbereitet und auch frühere Trennungserfahrungen dabei berücksichtigt. Explizit wird auf die selbst bzw. gemeinsam erarbeiteten Erfolge der Therapie verwiesen. Die 24. und 25. Sitzung dienen als „booster sessions“ und werden jeweils vier Wochen nach der letzten Sitzung anberaumt. Hier geht es um Inhalte wie Supervision, Verstärkung der Verbesserung des Patienten sowie Analyse von Rückfällen. Die eigene Arbeit des Patienten soll hervorgehoben und unterstützt werden.

\section{Fragestellung der vorliegenden Arbeit}

Aus den zuvor dargestellten Befunden wurde ersichtlich, dass der Erforschung des Therapieprozesses bisher noch nicht die Bedeutung beigemessen wird, die ihr aufgrund der Relevanz ihrer Befunde beigemessen werden müsste. V.a. die Forschergruppe um E. Jones hat mit Hilfe des PQS die Weiterentwicklung dieses Forschungszweigs im englischsprachigen Raum stark vorangetrieben. Im deutschen Sprachraum gibt es dazu bisher allerdings nur wenige Untersuchungen (z.B. Albani et al., 2000). Die vorliegende Studie soll deshalb 
weitere Erkenntnisse im Hinblick auf den Therapieprozess auch für den deutschen Sprachraum durch eine detaillierte Erforschung des Therapieprozesses ermöglichen.

Entsprechend der Überlegungen aus den vorangegangenen Abschnitten soll folgende Hauptfragestellung untersucht werden:

Gibt es Unterschiede zwischen Psychotherapieprozessen, die durch eine kognitivbehaviorale bzw. psychodynamische Psychotherapie bei Patienten mit einer Generalisierten Angststörung induziert werden? Liegen diese Unterschiede in den therapeutischen Interventionen und Verhaltensweisen, im Verhalten und emotionalen Zustand des Patienten, in den Interaktionen zwischen Patient und Therapeut oder in den in der Therapie fokussierten Inhalten? Gibt es Zusammenhänge zwischen dem Therapieprozess und dem Outcome der Therapie?

Aus dieser übergeordneten Hauptfragestellung werden verschiedene Unterfragestellungen abgeleitet, die im Rahmen der vorliegenden Studie mit Hilfe des PQS untersucht werden.

\section{Fragestellung 1: Unterscheiden sich zwei Sitzungen aus der mittleren Phase der Therapie bedeutsam voneinander?}

Vorangegangene Forschungsarbeiten (vgl. Ablon \& Jones, 1999, 2002; vgl. 3.3 in diesem Kapitel) hatten bereits gezeigt, dass es zwischen der 4. und 12. Sitzung einer Psychotherapie keine Unterschiede im Therapieprozess gibt. Aufgrund der inhaltlichen Fokussierung, die in den beiden Therapiemanualen zur Behandlung der GAS (vgl. 4.3.2 in diesem Kapitel) festgelegt ist, wird auch für diese Untersuchung erwartet, dass sich zwei Sitzungen aus der mittleren Phase einer Therapie weder in Bezug auf die Interaktionen zwischen Patient und Therapeut noch auf die therapeutischen Interventionen und die Haltung, das Verhalten oder den Zustand des Patienten bedeutsam voneinander unterscheiden. Dies soll sowohl für die gesamte Stichprobe als auch getrennt für die beiden Therapieansätze gelten.

\section{Fragestellung 2: Unterscheiden sich psychodynamisch und kognitiv-behavioral orientierte}

\section{Therapieprozesse bedeutsam voneinander?}

Die Erfahrungen aus der Psychotherapieforschung der letzten Jahrzehnte zeigten, dass es zur Beurteilung der vergleichenden Wirkung zweier Therapien wichtig ist, den Therapieprozess zu untersuchen. Erst mit diesem zusätzlichen Wissen, d.h. wenn bekannt ist, welche Prozessaspekte zu welchen Veränderungen führen, lassen sich eindeutigere 
Schlussfolgerungen bzgl. möglicher Differenzen zwischen verschiedenen Therapieansätzen ziehen.

Zusammenfassend lassen sich die Befunde der vergleichsweise wenigen vorliegenden Prozessstudien so beschreiben: Verhaltenstherapeuten im Vergleich zu psychodynamischen Therapeuten sind direktiver, aktiver und strukturierter, geben mehr Informationen, stellen mehr Fragen und legen ihren Fokus stärker auf Kognitionen und zukünftige Erwartungen; auch forcieren sie extratherapeutische Aktivitäten (z.B. Hausaufgaben) mehr.

Psychodynamische Therapeuten fokussieren stärker Emotionen, interpersonelle Beziehungen, vergangene Erfahrungen und unbewusstes Material. Außerdem sind sie im Vergleich zu Verhaltenstherapeuten zurückhaltender, geben weniger Ratschläge und reflektieren mehr (z.B. Sloane at al., 1975, Brunink \& Schroeder, 1979; Stiles et al., 1988; Jones \& Pulos, 1993; Wiser \& Goldfried, 1996, Goldfried et al., 1998, Ablon \& Jones, 1998, 1999; Blagys \& Hilsenroth, 2000, 2002). Hinsichtlich der Empathie und der Anzahl an Interpretationen gibt es keine Unterschiede.

In den Studien, die den Therapieprozess ebenfalls mit dem PQS untersuchten (z.B. Jones \& Pulos, 1993; Ablon \& Jones, 1999), zeigte sich, dass sich verschiedene Therapieansätze v.a. hinsichtlich therapeutischer Interventionen und Haltungen voneinander unterschieden. Das Verhalten und der emotionale Zustand des Patienten in der Therapie sowie die Interaktion zwischen Patient und Therapeut unterschieden sich nicht.

Aufgrund dieser Befunde wird für die vorliegende Untersuchung erwartet, dass sich die hier untersuchten psychodynamischen und kognitiv-behavioralen Therapieprozesse im Hinblick auf therapeutische Techniken und Haltungen bedeutsam voneinander unterscheiden, im Hinblick auf patientenseitige Merkmale und die Interaktion zwischen Patient und Therapeut jedoch nicht.

\section{Fragestellung 3: Stimmen die tatsächlich durchgeführten Therapien mit ihren jeweiligen Prototypen überein?}

Um valide Aussagen über die Wirksamkeit von Therapieansätzen treffen zu können, ist es wichtig, zu wissen und auch beschreiben zu können, welche zentralen Prozessvariablen (also z.B. welche therapeutischen Interventionen) innerhalb eines Therapieprozesses eingesetzt wurden. Behandlungsmanuale, die dem Therapeuten genaue Anweisungen und Materialien für die Therapie zur Verfügung stellen, erleichtern dies. Erst durch die Verwendung solcher Manuale sind Aussagen darüber möglich, wie stark sich die behandelnden Therapeuten an die 
Vorgaben, die im Manual festgelegt wurden, gehalten haben („Manualtreue“). Und erst über die Erfassung der Manualtreue ist es im Zusammenhang mit Prozessforschung möglich, die potentiellen „Wirkfaktoren“ der jeweiligen Therapie zu bestimmen. Denn so werden Aussagen darüber möglich, ob die Interventionen, die laut Therapietheorie zu Veränderungen im Patienten führen sollen und die gemäß Manual in der Therapie anzuwenden waren, auch tatsächlich zu den wirksamen Elementen der jeweiligen Therapie gehören.

Um dies für die vorliegende Untersuchung überprüfen zu können, sollen in einem ersten Schritt zunächst die beiden durchgeführten Therapien mit den bereits beschriebenen (vgl. 3.3 in diesem Kapitel) Prototypen von Ablon und Jones (1998) verglichen werden. So kann festgestellt werden, ob die Therapeuten auch tatsächlich so arbeiten, wie es prototypisch für eine kognitiv-behaviorale bzw. eine psychodynamische Therapie sein sollte. Aufgrund der Konzeptionalisierung der beiden hier implementierten Therapien und der damit verbundenen theoretischen Orientierung wird erwartet, dass die psychodynamische Therapie stärker dem psychodynamischen als dem kognitiv-behavioralen Prototyp und die kognitiv-behaviorale Therapie stärker dem kognitiv-behavioralen als dem psychodynamischen Prototyp ähnelt (vgl. Ablon \& Jones, 2002; Ablon et al., 2006). Es soll demnach die Übereinstimmung mit dem der implementierten Therapie theoretisch zugrunde liegenden Prototyp erfasst werden.

\section{Fragestellung 4: Sind die nach der kognitiv-behavioralen bzw. psychodynamischen}

\section{Veränderungstheorie für die Veränderungen beim Patienten verantwortlichen Aspekte} auch tatsächlich für die Veränderungen verantwortlich? Wirkt sich die Orientierung am jeweils eigenen Prototyp positiv auf das Therapieergebnis aus?

Nachdem bestimmt wurde, wie stark sich die implementierten Therapien an ihren Prototypen orientieren, soll dann in einem zweiten Schritt überprüft werden, ob die Orientierung am theoretisch zugehörigen Prototyp mit einem positiven Outcome zusammenhängt. Untersucht wird demnach, ob diejenigen Aspekte, die laut Therapietheorie die wirksamen Elemente innerhalb der Therapie darstellen sollen, auch tatsächlich mit den Veränderungen im Patienten zusammenhängen.

Bisherige Studien (z.B. Ablon \& Jones, 1998, 2002) zeigten, dass Therapeuten sich häufig Interventionen anderer Therapieansätze „,borgen“ und dass manchmal genau diese mit den Veränderungen im Patienten zusammenhängen. Obwohl die den Therapien zugrunde liegenden Theorien zum Teil also gegensätzliche Wirkmechanismen definieren, kommt es in der Praxis dennoch zu deutlichen Überlappungen zwischen verschiedenen Therapieansätzen 
in der Anwendung dieser Interventionen (vgl. Goldfried et al., 1998). Deshalb ist es wichtig, zu untersuchen, welche Interventionen tatsächlich wirken und ob diese effektiven Interventionen einerseits nur in einem bestimmten Therapieansatz angewendet werden und andererseits auch nur dort wirksam sind. Dazu wird der Zusammenhang zwischen den Korrelationen der implementierten Therapien mit den jeweiligen Prototypen und dem Outcome berechnet. Dies ermöglicht eine Aussage darüber, ob die Aspekte, die nach der jeweils spezifischen Veränderungstheorie für Veränderungen im Patienten verantwortlich sein sollen, es auch tatsächlich in der Praxis sind („Wirkfaktoren“). Aus einer theoretischen Perspektive heraus wäre zu erwarten, dass sich die theoretisch postulierten „Wirkfaktoren“ auch in der Praxis als solche zeigen. Da die Untersuchungen von Ablon und Jones (1998, 2002) jedoch dazu konträre Befunde erbrachten, soll die hier vorliegende Untersuchung weitere Klarheit erbringen.

Neben der jeweils therapiearmspezifischen Beurteilung des Einflusses des Zusammenhangs der Übereinstimmung der implementierten Therapie und dem Prototyp mit dem Outcome soll auch der Zusammenhang der beiden Variablen „Festhalten am eigenen Prototyp“ und „Outcome“ bestimmt werden. Damit ist eine Aussage darüber möglich, inwiefern die Orientierung an therapeutischen Interventionen und Verhaltensweisen, die gemäß Therapietheorie generell zu einer „lege artis“ durchgeführten Therapie gehören, sich positiv auf den Therapieerfolg auswirkt.

\section{Fragestellung 5: Sind bestimmte Prozessmerkmale, die mit dem PQS erfasst werden,} signifikante Prädiktoren des Therapieerfolgs? Beschreiben diese prädiktiven Prozessvariablen eher Merkmale des Patienten oder des Therapeuten oder ihrer Interaktion? Gibt es spezifische Prädiktoren (Wirkfaktoren)?

In diesem Zusammenhang soll untersucht werden, ob bestimmte Therapieprozessmerkmale, die mit dem PQS erfasst werden, den Therapieerfolg vorhersagen können (vgl. Jones et al., 1988). Ablon und Jones (1999) z.B. fanden, dass v.a. Prozessmerkmale, die das Verhalten oder den emotionalen Zustand des Patienten in der Therapie beschreiben, den Therapieerfolg vorhersagen konnten. Werden diese Prädiktoren zwischen verschiedenen Therapieansätzen miteinander verglichen, lassen sich Rückschlüsse auf spezifische und unspezifische Wirkfaktoren ziehen. Insgesamt sind bisher keine eindeutigen Aussagen darüber möglich, ob spezifische oder unspezifische Faktoren den größeren Einfluss ausüben, da einige Studien den Einfluss unspezifischer Faktoren (z.B. 
Luborsky et al., 1975; Frieswyk et al., 1986; Castonguay, Goldfried, Wiser, Raue \& Hayes, 1996), andere hingegen den spezifischer Techniken und Interventionen zeigen konnten (z.B. Andrews \& Harvey, 1981; Landmann \& Dawes, 1982). Daher ist weitere Forschung auf diesem Gebiet notwendig.

In der vorliegenden Studie soll untersucht werden, ob eher patienten- oder eher therapeutenseitige Merkmale oder solche der Interaktion zwischen Patient und Therapeut das Outcome vorhersagen können und ob es Unterschiede in den Prädiktoren zwischen den beiden Therapiearmen gibt. Außerdem soll verglichen werden, ob diejenigen Prozessmerkmale, die sich als Prädiktoren des Therapieerfolgs herausstellen, bedeutsam zwischen den beiden Therapiearmen unterscheiden (vgl. Fragestellung 2). 
Methode

Beschreibung der Stichprobe

\section{Methode}

\section{Beschreibung der Stichprobe}

\subsection{Patienten}

Die in dieser Arbeit untersuchte Stichprobe von $\mathrm{N}=42$ Patienten setzt sich aus Teilnehmern einer von der Deutschen Forschungsgemeinschaft geförderten längsschnittlichen, randomisierten, kontrollierten Studie zur Behandlung der GAS (,Wirkfaktoren psychodynamischer Kurzzeittherapie und kognitiver Verhaltenstherapie bei Generalisierter Angststörung“") der Abteilung Psychosomatik und Psychotherapie des Universitätsklinikums Göttingen zusammen. Die Teilnehmer wurden über Annoncen in den Lokalzeitungen, über öffentliche Aushänge, über Informationsveranstaltungen sowie durch Überweisungen niedergelassener Psychotherapeuten und Ärzte für die Studie gewonnen.

Zur diagnostischen Klassifikation einer GAS als Hauptdiagnose (300.02 nach DSM-IV, F41.1 nach ICD-10) wurden das an der ICD-10 orientierte Mini-DIPS (Margraf, 1994) sowie das SKID II (Wittchen, Zaudig \& Fydrich, 1997) eingesetzt. Falls die Hauptdiagnose, d.h. die mit der gegenwärtig die schwerste Beeinträchtigung verbunden ist, eine depressive Episode, eine phobische, eine Panik- oder Zwangsstörung war, wurde dieser gemäß ICD-10 Priorität gegenüber der GAS-Diagnose eingeräumt (vgl. 4.1 in Kapitel II).

In die Studie wurden ausschließlich Patienten zwischen 18 und 65 Jahren aufgenommen. Folgende Ausschlusskriterien wurden definiert:

1. Vorliegen einer akuten oder schweren körperlichen Erkrankung, die der Durchführung der Studie zuwider laufen könnte.

2. Vorliegen einer gegenwärtigen oder zurückliegenden schizophrenen, bipolaren oder Achse II -Persönlichkeitsstörung des Clusters A (schizoid, schizotypisch, paranoid).

3. Vorliegen eines gegenwärtigen oder zurückliegenden Anfallsleidens sowie Vorliegen einer klinisch bedeutsamen hirnorganischen Erkrankung.

4. Erfüllung der Kriterien für eine Alkohol- oder Substanz-Abhängigkeit oder für einen Alkohol- oder Substanzmissbrauch, für eine Essstörung, für eine „Major Depression“, für eine rezidivierende depressive Störung oder für eine BorderlinePersönlichkeitsstörung innerhalb der letzten 12 Monate.

5. Anderweitige psychotherapeutische oder psychopharmakologische Behandlung. 
Patienten mit partiell remittierter Depression im Rahmen einer rezidivierenden depressiven Erkrankung sowie Patienten mit Substanzmissbrauch in der Vorgeschichte wurden jedoch zugelassen.

Insgesamt wurden 231 Patienten gescreent, 52 davon erhielten die Hauptdiagnose GAS und wurden in die Studie aufgenommen. Davon waren 42 (80,8 \%) Frauen. 27 Patienten (51,9 $\%, 77 \%$ Frauen) wurden dem kognitiv-verhaltenstherapeutischen Behandlungsarm randomisiert zugeteilt; 25 (84 \% Frauen) der psychodynamischen Behandlung.

Alle Therapiesitzungen wurden auf Tonband aufgezeichnet. Für die vorliegende Arbeit wurden zur Untersuchung des Therapieprozesses von jedem Patienten eine „frühere“ (10.) und eine „spätere“ (18.) Sitzung mit dem PQS analysiert. Da nach beiden Therapie-Manualen (vgl. 4.3.2 in Kapitel II) in den ersten Sitzungen vor allem diagnostische und anamnestische und in den späten Sitzungen vor allem rückfallpräventive Aspekte thematisiert werden sollen, wurden als Basis der Therapieprozessanalyse Sitzungen aus dem mittleren Teil der Therapie ausgewählt.

Insgesamt konnten Sitzungsbänder von $\mathrm{N}=42$ der 52 Patienten analysiert werden, sodass 84 Sitzungen zu beurteilen waren. Bei einem der Patienten wurde die Therapie bereits nach der 15. Sitzung abgeschlossen, ein weiterer Patient brach die Therapie ab. Bei zwei Patienten war die Qualität der Sitzungsbänder so mangelhaft, dass sie nicht verwendet werden konnten und bei sechs Patienten fehlten die entsprechenden Sitzungsbänder.

Das durchschnittliche Alter der aus 42 Patienten bestehenden Stichprobe mit analysierbaren Sitzungsbändern lag zu Beginn der Therapie bei 44,4 Jahren ( $\mathrm{sd}=12,303$, Range 21 - 65 Jahre), wobei sich weder Männer und Frauen noch die beiden Behandlungsarme im Alter signifikant voneinander unterschieden $(\mathrm{p}=.871 \mathrm{bzw} . \mathrm{p}=.531)$.

Von diesen 42 Patienten waren 19 Patienten (darunter 15 Frauen) dem psychodynamischen und 23 (darunter 18 Frauen) dem kognitiv-behavioralen Behandlungsarm durch Randomisierung zugeteilt worden. Zur Randomisierung wurden vor Beginn der Studie erstellte Randomisierungslisten verwendet, in denen die Zuordnung zu den beiden Behandlungsgruppen vorab für alle Teilnehmer anhand einer fortlaufenden Nummerierung von 1-60 festgelegt war. Diese Randomisierungslisten wurden unter Verschluss gehalten und durch einen unabhängigen Mitarbeiter verwaltet.

$54,8 \%$ der Untersuchungsstichprobe hatten keine psychotherapeutische und 92,9\% keine psychiatrische Vorbehandlung. Männer und Frauen unterschieden sich weder in der psychotherapeutischen $(p=.26)$ noch in der psychiatrischen Vorbehandlung $(p=1.00)$ 
signifikant voneinander. Auch im Hinblick auf die beiden Behandlungsarme zeigten sich weder in der psychotherapeutischen $(\mathrm{p}=.11)$ noch in der psychiatrischen $(\mathrm{p}=.44)$ Vorbehandlung signifikante Unterschiede. Die Therapien wurden zwischen 2001 und 2005 durchgeführt.

Die Hälfte der Patienten (21) ist verheiratet, weitere 17 sind ledig (vgl. Tab. 4), wobei hier der Anteil unter den Männern doppelt so hoch ist wie unter den Frauen (66,7 vs. 33,3 \%). Die beiden Teilstichproben unterscheiden sich hierin nicht bedeutsam voneinander $(p=.20)$. Fast $80 \%$ der Patienten leben in einer festen Partnerschaft (vgl. Tab. 4), wobei die Patienten aus der psychodynamisch behandelten Therapiegruppe hier signifikant häufiger vertreten sind ( $p$ $=.02$ ). Fast ein Drittel der Patienten hat gar keine Kinder, der Rest zwischen einem und vier (vgl. Tab. 4), wobei sich die Behandlungsarme in der durchschnittlichen Kinderanzahl nicht signifikant voneinander unterscheiden $(\mathrm{p}=.96)$. Knapp die Hälfte aller Patienten $(47,6 \%)$ hat das Abitur, rund zwei Drittel haben eine Lehre abgeschlossen (vgl. Tab. 4). Über 70 \% (71,4 \%) arbeiten oder sind berentet, der Rest lebt von den Eltern oder vom Partner. 61,9 \% arbeiten in einem Angestelltenverhältnis (vgl. Tab. 4). Nur zwei der 42 Patienten waren zu Behandlungsbeginn krank geschrieben, über $95 \%$ arbeiteten regulär. Auch in den letzten zwölf Monaten vor Behandlungsbeginn waren über 50 \% nicht arbeitsunfähig, der Rest nicht länger als 3 Monate (vgl. Tab. 4). Die Patienten der beiden Behandlungsarme unterschieden sich in keinem einzigen dieser Merkmale signifikant voneinander. Allerdings zeigte sich, dass die Patienten in der kognitiv-behavioralen Stichprobe deutlich häufiger voll und deutlich seltener nicht nicht erwerbstätig waren als die in der psychodynamischen Stichprobe ( $\mathrm{p}=$ .02). Auch Männer und Frauen unterscheiden sich hierin voneinander. Während 77,8 \% der Männer in einer Vollzeitstelle arbeiten, tun dies bei den Frauen nur 33,3 \%.

Nur 13 (31\%) der 42 Patienten hatten keine weiteren komorbiden Störungen auf Achse I (vgl. Tab. 4). Sechs der 42 Patienten zeigten neben der GAS-Diagnose, die allerdings als Hauptdiagnose, d.h. als beeinträchtigender angesehen wurde, auch eine depressive Episode, eine dysthyme Störung oder eine verlängerte depressive Reaktion. Weitere 13 wiesen eine zweite Angststörung auf (z.B. Soziale oder Spezifische Phobie). Bei drei Patienten zeigten sich Zwangsgedanken oder -handlungen; weitere drei hatten ein somatoforme Störung. Ein Patient zeigte außerdem Essattacken, die im Zusammenhang mit einer sonstigen psychischen Störung standen. 26 \% der Patienten zeigten außerdem noch eine dritte Achse-I-Störung. Auch hier standen depressive und andere Angststörungen im Vordergrund (vgl. Tab. 4). 
Methode

Beschreibung der Stichprobe

Tabelle 4: Soziodemographische Daten der Untersuchungsstichprobe.

\begin{tabular}{|c|c|c|}
\hline Merkmale & $\begin{array}{l}\text { Psychodynamischer } \\
\text { Behandlungsarm }(\mathrm{n}=19)\end{array}$ & $\begin{array}{l}\text { Kognitiv-behavioraler } \\
\text { Behandlungsarm }(n=23)\end{array}$ \\
\hline Geschlecht & $\begin{array}{l}78,9 \% \text { (15) Frauen } \\
21,1 \% \text { (4) Männer }\end{array}$ & $\begin{array}{l}78,3 \% \text { (18) Frauen } \\
21,7 \% \text { (5) Männer }\end{array}$ \\
\hline Alter & 43,05 Jahre $(\mathrm{sd}=13,85)$ & $45,48(\mathrm{sd}=11,07)$ \\
\hline $\begin{array}{l}\text { Familienstand } \\
\text { ledig } \\
\text { verheiratet } \\
\text { getrennt } \\
\text { geschieden }\end{array}$ & $\begin{array}{l}36,8 \%(7) \\
63,2 \%(12)\end{array}$ & $\begin{array}{l}43,5 \%(10) \\
39,1 \%(9) \\
8,7 \%(2) \\
8,7 \%(2)\end{array}$ \\
\hline $\begin{array}{l}\text { Partnerschaft } \\
\text { ja } \\
\text { nein }\end{array}$ & $\begin{array}{l}94,7 \%(18) \\
5,3 \%(1)\end{array}$ & $\begin{array}{l}65,2 \%(15) \\
34,8 \%(8)\end{array}$ \\
\hline $\begin{array}{l}\text { Anzahl Kinder } \\
0 \\
1 \\
2 \\
3 \\
4 \\
\text { MW }\end{array}$ & $\begin{array}{l}31,6 \%(6) \\
21,1 \%(4) \\
36,8 \%(7) \\
10,5 \%(2) \\
1,26(\mathrm{sd}=1,05)\end{array}$ & $\begin{array}{l}30,4 \%(7) \\
26,1 \%(6) \\
34,8 \%(8) \\
4,3 \%(1) \\
4,3 \%(1) \\
1,26(\mathrm{sd}=1,1)\end{array}$ \\
\hline $\begin{array}{l}\text { Schulabschluss } \\
\text { Hauptschule } \\
\text { Mittlere Reife } \\
\text { Abitur }\end{array}$ & $\begin{array}{l}10,5 \%(2) \\
31,6 \%(6) \\
57,9 \% 11)\end{array}$ & $\begin{array}{l}17,4 \%(4) \\
43,5 \%(10) \\
39,1 \%(9)\end{array}$ \\
\hline $\begin{array}{l}\text { Berufsabschluss } \\
\text { Ohne } \\
\text { Lehre } \\
\text { Uni }\end{array}$ & $\begin{array}{l}15,8 \%(3) \\
47,4 \%(9) \\
36,8 \%(7)\end{array}$ & $\begin{array}{l}4,3 \%(1) \\
73,9 \%(17) \\
21,7 \%(5)\end{array}$ \\
\hline $\begin{array}{l}\text { Einkommensquelle } \\
\text { Arbeit/Rente } \\
\text { Eltern/Partner }\end{array}$ & $\begin{array}{l}57,9 \%(11) \\
42,1 \%(8)\end{array}$ & $\begin{array}{l}82,6 \%(19) \\
17,4 \%(4) \\
\end{array}$ \\
\hline $\begin{array}{l}\text { Berufstätigkeit } \\
\text { Angestellt } \\
\text { Selbständig } \\
\text { Azubi } \\
\text { Student } \\
\text { Hausmann } \\
\text { Rentner } \\
\end{array}$ & $\begin{array}{l}47,4 \%(9) \\
5,3 \%(1) \\
5,3 \%(1) \\
15,8 \%(3) \\
21,2 \%(4) \\
5,3 \%(1) \\
\end{array}$ & $\begin{array}{l}73,9 \%(17) \\
8,7 \%(2) \\
4,3 \%(1) \\
13 \%(3)\end{array}$ \\
\hline $\begin{array}{l}\text { Erwerbstätigkeit } \\
\text { Voll } \\
\text { Teilzeit } \\
\text { nicht }\end{array}$ & $\begin{array}{l}21,1 \%(4) \\
36,8 \%(7) \\
42,1 \%(8)\end{array}$ & $\begin{array}{l}60,9 \%(14) \\
26,1 \%(6) \\
13 \%(3)\end{array}$ \\
\hline $\begin{array}{l}\text { Dauer der aktuellen AU (in } \\
\text { Wochen) } \\
0 \\
3\end{array}$ & $\begin{array}{l}94,7 \%(18) \\
5,3 \%(1)\end{array}$ & $\begin{array}{l}95,7 \%(22) \\
4,3 \%(1)\end{array}$ \\
\hline $\begin{array}{l}\text { AU in letzten } 12 \text { Monaten } \\
\text { Keine } \\
\text { Unter } 3 \text { Monate }\end{array}$ & $\begin{array}{l}47,4 \%(9) \\
52,6 \%(10)\end{array}$ & $\begin{array}{l}56,5 \%(13) \\
43,5 \%(10)\end{array}$ \\
\hline
\end{tabular}

\subsection{Therapeuten}

Insgesamt behandelten neun Psychotherapeuten die Patienten in der GAS-Studie. Drei von ihnen hatten eine verhaltenstherapeutische, sechs eine psychoanalytische oder 
tiefenpsychologische Ausbildung. Alle Therapeuten arbeiteten als niedergelassene Psychotherapeuten. Acht von ihnen (88,9 \%) konnten zum Zeitpunkt des Studienbeginns 2001 auf eine langjährige Berufserfahrung von mindestens neun Jahren zurückblicken. Die durchschnittliche Berufserfahrung lag bei 16,44 Jahren, mit einem Range zwischen 4 und 26 Jahren. Die Therapeuten beider Therapiegruppen unterschieden sich hierin nicht signifikant voneinander. Die Therapeuten behandelten zwischen einem und elf Patienten.

Zu Beginn der Studie lag das Alter der Therapeuten im Durchschnitt bei 47,88 Jahren mit einem Range von 41 bis 53 Jahren. Auch hier gab es keine signifikanten Unterschiede zwischen den psychodynamisch und den kognitiv-behavioral orientierten Therapeuten. Drei Therapeuten (33,3\%) waren weiblichen Geschlechts.

Die Therapeuten wurden persönlich von den Organisatoren der Studie angeworben. Vor Beginn der eigentlichen Therapien durchliefen sie ein manualgestütztes Training. Zunächst erhielten sie dazu eine theoretische Einführung in das Manual und behandelten dann ein bis zwei „Trainingsfälle“. Probleme in der Probebehandlung wurden mit den Autoren des jeweiligen Therapiemanuals besprochen. Um sicherzustellen, dass die Therapeuten sich an das im Manual beschriebene Vorgehen hielten, wurden sie in regelmäßigen Abständen von erfahrenen Supervisoren supervidiert. Beides zusammen- die Benutzung von Behandlungsmanualen sowie die Supervision- sollte die Therapieintegrität sichern (vgl. Moncher \& Prinz, 1991).

\section{Messinstrumente zur Erfassung diagnose- und outcome-relevanter Maße}

\subsection{Fremdbeurteilungsmaße}

Zunächst wurden die für die Studie in Frage kommenden Patienten zu zwei ScreeningTerminen eingeladen, in denen geprüft wurde, ob sie die definierten Einschlusskriterien (vgl. 1. in diesem Kapitel) erfüllten. Je ein unabhängiger Diagnostiker, der nicht an den späteren Therapien beteiligt war, führte das Mini-DIPS (Margraf, 1994; vgl. 1.1 in diesem Kapitel) und das SKID-II (Wittchen et al., 1997) durch. Die für den psychotherapeutischen Bereich relevanten psychischen Störungen der Achse I (nach DSM-IV, Saß, Wittchen \& Zaudig, 2000) wurden mit Hilfe des Mini-DIPS (Margraf, 1994) erfasst, dessen Interrater-Reliabilität mit einem kappa von .84 bis 1.0 angegeben wird (Margraf, 2002). Evtl. vorliegende Persönlichkeitsstörungen (Achse-II-Störungen) wurden mit dem SKID-II (Wittchen et al., 1997), das sich ebenfalls am DSM-IV orientiert, diagnostiziert. Hier liegt der Median der Interrater-Kappa-Koeffizienten bei .70 (Wittchen, Zaudig \& Fydrich, 2002). 
Methode

Messinstrumente

Zur Erfassung der Angst- bzw. der häufig komorbid auftretenden depressiven Symptomatik wurden außerdem die Hamilton Angst Skala (HAMA, Hamilton, 1976) und die Hamilton Depression Skala (HAMD, Hamilton, 1986) verwendet. Diese wurden, um die Reliabilität der Diagnose sicherstellen zu können, von mehreren Diagnostikern ausgewertet, die zunächst ein Training in der Anwendung dieser beiden Instrumente durchliefen. Bei diagnostisch schwierigen Fällen wurde eine Konsensus-Entscheidung zwischen zwei oder drei der trainierten Diagnostiker getroffen.

Die HAMA und die HAMD stellen weit verbreitete Instrumente zur Fremdbeurteilung des Schweregrads einer Angststörung bzw. einer Depression dar (Hautzinger, 2002b, c). Voraussetzung für ihre Anwendung ist, dass bereits eine Angststörung und/oder Depression diagnostiziert wurden. Die Beurteilung erfolgt durch erfahrene und trainierte Diagnostiker auf der Basis eines ausführlichen klinischen Interviews (ca. 30 Minuten), in dessen Verlauf der Diagnostiker durch Patientenaussagen, durch eigene Beobachtungen sowie evtl. auch durch Fremdeinschätzungen von z.B. Angehörigen oder Pflegepersonal zu einer Einschätzung bzgl. des Schweregrads der Störungen gelangt. Bezugszeitraum ist die Woche vor dem klinischen Interview.

Die HAMA besteht aus 14 Items, die sich auf psychische und somatische Aspekte des Angstsyndroms beziehen: erfasst werden u.a. ängstliche und depressive Stimmung, Furcht, Schlaflosigkeit sowie somatische Symptome. Jedes Item wird auf einer Skala von, $0=$ nicht vorhanden“ bis , ,4 = sehr stark vorhanden“ beurteilt; psychische und somatische Items werden getrennt voneinander addiert und als zwei Skalen (somatische und psychische Angst) interpretiert. Daneben wird ein Gesamtwert als globales Maß der Angst angegeben. Erfahrungsgemäß weisen Patienten mit der Diagnose einer GAS zu Beginn ihrer Behandlung typischerweise Werte zwischen 25 und 26 Punkten bei der HAMA auf, nach vierwöchiger Behandlung sinken diese auf Werte von 10 Punkten (Hautzinger, 2002b). Korrelationen mit anderen Angstmaßen liegen zwischen .32 und .93 (Hautzinger, 2002b). Normwerte aufgrund von Eichstichproben existieren nicht (Hautzinger, 2002b).

Bei der HAMD steht die Erfassung somatischer, motorischer sowie psychotischer Symptome, Tagesschwankungen und Krankheitseinsicht im Vordergrund. Folgende Symptombereiche werden zur Beurteilung der Schwere der Symptomatik vorgegeben: depressive Stimmung und Hemmung, Schuldgefühle, Suizidneigung, Schlafstörungen, Beeinträchtigungen bei der Arbeit/Tätigkeiten, Erregung, psychische und somatische Angst, körperliche Symptome (gastrointestinale Schmerzen, Erschöpfung, Vitalgefühl), 
Methode

Messinstrumente

Genitalsymptome, Hypochondrie, Gewichtsveränderungen, Krankheitseinsicht,

Tagesschwankungen, Derealisation/Depersonalisation, paranoide und Zwangssymptome. Sie besteht aus 21 Items, wobei für 9 Items eine fünfstufige, für ein Item eine vierstufige und für 11 Items eine dreistufige Skala zur Beurteilung vorgegeben wird. Die Einzelurteile werden aufaddiert, sodass ein Wert zwischen 0 und 82 Punkten resultiert. Normwerte existieren nicht, allerdings können aufgrund der umfangreichen Erfahrungen Erwartungs- und Grenzwerte zu Behandlungsbeginn, für den Verlauf und bei Behandlungsende angegeben werden (Hautzinger, 2002c). So zeigen depressive Patienten erfahrungsgemäß meist Werte zwischen 24 und 30 Punkten (Hautzinger, 2002c), nicht-depressive Patienten liegen deutlich darunter. Bei weitgehender Remission der Depression können am Ende einer Behandlung Werte von unter 8 erwartet werden. Korrelationen mit anderen Depressionsmaßen wie z.B. dem BDI liegen zwischen .16 und .82 (Hautzinger, 2002c).

\subsection{Selbstbeurteilungsmaße}

\section{Instrument zur Erfassung der depressiven Symptomatik}

Das Beck Depression Inventar (BDI; Hautzinger, Bailer, Worrall \& Keller, 2000) ist ein Selbstbeurteilungsinstrument zur Erfassung der Schwere der depressiven Symptomatik bei Patienten aus klinischen Populationen. Sehr häufig wird es als Erfolgs- und Verlaufsmaß bei jeglicher Art von Interventionsforschung eingesetzt (Hautzinger, 2002a). Es eignet sich für Patienten zwischen 16 und 80 Jahren und besteht aus 21 Gruppen von Items, die typische depressive Symptome beschreiben (Dysphorie, Pessimismus, Versagen, Unzufriedenheit, Schuldgefühle, Strafbedürfnis, Selbsthass, Selbstanklagen, Selbstmordimpulse, Weinen, Reizbarkeit, Rückzug und Isolation, Entschlussunfähigkeit, negatives Körperbild, Arbeitsunfähigkeit, Schlafstörungen, Ermüdbarkeit, Appetitverlust, Gewichtsverlust, Hypochondrie, Libidoverlust). Motorische Auffälligkeiten, Gewichtszunahme und gesteigertes Schlafbedürfnis werden nicht berücksichtigt. Jede dieser 21 Gruppen besteht aus vier Aussagen, die nach aufsteigender Schwere und zunehmender Beeinträchtigung gestaffelt sind und die die Patienten von ,0 = nicht vorhanden“ bis, $3=$ stark vorhanden“ beurteilen sollen. Die Werte der Einzelurteile werden addiert, wobei der entstehende Summenwert als Ausdruck des Schweregrads der gegenwärtigen depressiven Symptomatik zu interpretieren ist. Werte über 18 gelten als klinisch signifikant (Hautzinger, 2002a); depressive Patienten zeigen typischerweise Werte über 20, depressive Patienten in Remission und klinisch unauffällige Kontrollpatienten Werte unter 9 (Hautzinger, 2002a). Korrelationen mit anderen 
Methode

Messinstrumente

Selbstbeurteilungsinstrumenten depressiver Symptome liegen zwischen .79 und .89 (Hautzinger, 2002a).

\section{Instrumente zur Erfassung der Angstsymptomatik}

Das Beck Angst Inventar (BAI) misst die Schwere klinisch relevanter Ängste in Patientengruppen und in der Allgemeinbevölkerung (Margraf \& Ehlers, 2003). Vorteil dieses Instruments ist, dass es weniger stark mit Depressivität korreliert als andere Fragebogenverfahren zur Erfassung der Angst. Es besteht aus 21 Items, die sich mehrheitlich an den Symptomlisten des DSM zur Klassifikation von Panikanfällen und generalisierter Angst orientieren. Die Probanden sollen diese Items auf einer vierstufigen Skala hinsichtlich der Schwere ihres Auftretens in den letzten 7 Tagen einschätzen. Die Einzelwerte werden addiert, wobei Werte zwischen 0 - 63 Punkten resultieren können. Patienten mit Angststörungen zeigen typischerweise einen Wert von mindestens 23,5, Personen der Allgemeinbevölkerung hingegen von 3,5 (Margraf \& Ehlers, 2003). Das BAI korreliert mit konstruktnahen Instrumenten wie dem STAI-T (s.u.) zu .48, mit dem STAI-S zu .45 und mit Depressionsmaßen wie dem BDI zu .47 (Margraf \& Ehlers, 2003).

Das State Trait Angst Inventar (STAI; Laux, Glanzmann, Schaffner \& Spielberger, 2002) ist ein Selbstbeschreibungsverfahren für Personen zwischen 15 und 75 Jahren, das sich für die Grundlagenforschung, die Persönlichkeitsdiagnostik, die klinische Diagnostik der Angstneigung sowie für die Verlaufskontrolle von Angstzuständen bei therapeutischen Interventionen und pharmakologischen Behandlungen eignet (Laux et al., 2002). Es besteht aus zwei unabhängigen Skalen mit je 20 Feststellungen, deren Bearbeitung jeweils etwa 5 Minuten dauert. Das STAI-S erfasst Angst als Zustand (,state“) auf einer vierstufigen Intensitätsskala und kann daher den Angstverlauf innerhalb einer therapeutischen Sitzung ebenso wie über die gesamte Therapie hinweg erfassen. Das STAI-T misst Ängstlichkeit als Eigenschaft (,trait“) auf einer vierstufigen Häufigkeitsskala und ermöglicht so eine Indikationsdiagnostik, eine Therapieevaluation sowie eine Vorhersage des Therapieerfolgs. Ebenso ermöglicht es die Diagnose neurotischer Störungen und die Differenzierung von generalisierten Ängsten und spezifischen Phobien. Für jede der beiden Skalen wird ein Summenwert gebildet, der zwischen 20 und 80 variieren kann. Normen liegen naturgemäß nur für das STAI-T vor (Margraf \& Ehlers, 2003), das zu .80 mit dem BDI korreliert (Margraf \& Ehlers, 2003).

Der Penn State Worry Questionnaire (PSWQ; Meyer, Miller, Metzger \& Borkovec, 1990) ist ein Selbstbeurteilungsinstrument mit 16 Items, der das Ausmaß an pathologischer 
Methode

Messinstrumente

Besorgnis bei Personen ab 18 Jahren erfasst (Stöber \& Bittencourt, 2002). Pathologische Besorgnis (chronische, exzessive und unkontrollierbare Besorgnis) ist das Hauptkriterium für die Diagnose einer Generalisierten Angststörung. Die mit dem PSWQ gewonnenen Daten werden zur Therapieprozessdiagnostik und zur Therapieevaluation, nicht jedoch zur Indikationsstellung eingesetzt. Die 16 Items des PSWQ können in ca. 5 Minuten bearbeitet werden, wobei die Person angibt, wie typisch diese Aussagen, die sich auf die pathologische Besorgnis beziehen, für sie sind; der PSWQ eignet sich deshalb nicht zur Veränderungsmessung, da er eine situationsübergreifende und zeitstabile Eigenschaft misst. Der PSWQ-PW (Penn State Worry Questionnaire - Past Week) hingegen, der aus nur 15 Items besteht, bezieht sich nur auf die vergangene Woche. Er dient daher der Änderungs- und Verlaufsdiagnostik. Die Items werden auf einer siebenstufigen Skala von, $0=$ nie“ bis, $6=$ fast immer" beantwortet, diese Antworten werden addiert und liefern so einen Wert für die pathologische Besorgnis (während der letzten Woche). Es existieren keine Normwerte, jedoch zeigt sich ein prototypisches Befundmuster, wie es für ein valides und änderungssensitives Instrument zur Erfassung pathologischer Besorgnis zu erwarten ist (Stöber \& Bittencourt, 2002).

\section{Instrument zur Erfassung Interpersonaler Probleme}

Das Inventar Interpersonaler Probleme (IIP; Horowitz, Strauß \& Kordy, 2000) ermöglicht es, Probleme zu beschreiben, die sich auf spezifische Schwierigkeiten im Umgang mit anderen Menschen beziehen (Horowitz, Strauß \& Kordy, 2002). Es wurde v.a. für die Anwendung im Erwachsenenbereich zur klinischen Individual- und Verlaufsdiagnostik sowie zur Erfolgskontrolle in der Psychotherapieforschung konzipiert. In der zugrunde liegenden Theorie wird davon ausgegangen, dass alle interpersonalen Verhaltensweisen entlang der Achsen eines zweidimensionalen Raums beschreibbar sind: Achse 1 „Zuneigung oder Fürsorge“ reicht von sehr feindseligem zu sehr freundlichem oder liebevollem Verhalten, Achse 2 „Macht, Kontrolle, Dominanz“ von unterwürfigem zu dominantem Verhalten. Es werden mit den 64 Items, deren Bearbeitung etwa 15 Minuten in Anspruch nimmt, acht Skalen erfasst: zu autokratisch/dominant, zu streitsüchtig/konkurrierend, zu abweisend/kalt, zu introvertiert/sozial vermeidend, zu selbstunsicher/unterwürfig, zu ausnutzbar/nachgiebig, zu fürsorglich/freundlich und zu expressiv/aufdringlich. Normen liegen getrennt nach Alter und Geschlecht vor. Untersuchungen zeigen, dass das IIP änderungssensitiv ist, sich diese Änderungen aber erst in der Katamnese zeigen und dass das Ausmaß an berichteten interpersonalen Problemen ein gutes Prognosekriterium ist (Horowitz et al., 2002). 
Tabelle 5 zeigt die Variablen und Messinstrumente, die zur diagnostischen Kennzeichnung der Patienten zu Eingang in die Therapie und als Outcome-Kriterium verwendet wurden. Als Kriterium für den Therapieerfolg wird jeweils die Differenz zwischen dem Prä- und dem Post-Wert herangezogen. Das Mini-DIPS sowie das SKID-II wurden ausschließlich zur Diagnosestellung und zur Ausschlusskriterienbestimmung eingesetzt und sind daher in der Tabelle nicht erwähnt. Als Prozessvariablen dienen die Ratings des PQS, die von der 10. und der 18. Therapiestunde erhoben wurden.

Tabelle 5: Überblick über die verwendeten Messinstrumente.

\begin{tabular}{|l|l|}
\hline Variable & Messinstrument \\
\hline Angst & HAMA* \\
& BAI \\
& STAI \\
& PSWQ \\
\hline Depressivität & HAMD* \\
& BDI \\
\hline Interpersonale Schwierigkeiten & IIP \\
\hline
\end{tabular}

$*=$ Fremdbeurteilung

\section{Erfassung des Therapieprozesses: Das Psychotherapy Process Q-Sort (PQS)}

Das PQS wird in der vorliegenden Untersuchung zur Fremd-Beurteilung eines Audiomitschnittes der 10. bzw. 18. Therapiesitzung der 42 Patienten eingesetzt. Ein Manual gibt genaue Instruktionen, wie beim Codieren vorzugehen ist (vgl. Anhang A). Das Instrument besteht aus insgesamt 100 Items, die drei verschiedenen Kategorien zuzuordnen sind:

1. Items, die die Haltung, das Verhalten oder die Erlebnisse des Patienten beschreiben („Die Patientin ist ängstlich oder angespannt.“).

2. Items, die die Handlungen und Haltungen des Therapeuten widerspiegeln (,Der Therapeut ist distanziert, unbeteiligt.").

3. Items, die die Art der Interaktion der Dyade bzw. das Klima oder die Atmosphäre der Begegnung erfassen können (,Die therapeutische Beziehung hat eine erotische Färbung.“).

Die Rater hören sich den kompletten Mitschnitt der zu beurteilenden Therapiestunde an. Sie ordnen dann jede der 100 Aussagen zum Therapieprozess in einem „erzwungenen“ 
Methode

Psychotherapy Process Q-Sort

Verteilungsverfahren (vgl. 3.2 in Kapitel II) einer von neun Antwortkategorien auf einer

Ratingskala zu (vgl. Tab. 6). Die Beurteiler entscheiden mit ihren Ratings also über die Intensität und die geschätzte Wichtigkeit jedes einzelnen Items im Vergleich zu den restlichen 99 Items.

Tabelle 6: Bedeutung der Bewertungskategorien des PQS und Häufigkeit ihrer erlaubten Belegung.

\begin{tabular}{|l|l|l|}
\hline Kategorie & Bedeutung & Häufigkeit der Belegung \\
\hline 1 & $\begin{array}{l}\text { extrem uncharakteristisch oder } \\
\text { nicht zutreffend }\end{array}$ & 5 \\
\hline 2 & $\begin{array}{l}\text { ziemlich uncharakteristisch } \\
\text { oder nicht zutreffend }\end{array}$ & 8 \\
\hline 3 & $\begin{array}{l}\text { Einigermaßen } \\
\text { uncharakteristisch oder nicht } \\
\text { zutreffend }\end{array}$ & 12 \\
\hline 4 & $\begin{array}{l}\text { Ein wenig uncharakteristisch } \\
\text { oder nicht zutreffend }\end{array}$ & 16 \\
\hline 5 & Relativ neutral oder unwichtig & 18 \\
\hline 6 & $\begin{array}{l}\text { Ein wenig charakteristisch oder } \\
\text { zutreffend }\end{array}$ & 16 \\
\hline 7 & $\begin{array}{l}\text { Einigermaßen charakteristisch } \\
\text { oder zutreffend }\end{array}$ & 12 \\
\hline 8 & $\begin{array}{l}\text { Ziemlich charakteristisch oder } \\
\text { zutreffend }\end{array}$ & 8 \\
\hline 9 & $\begin{array}{l}\text { Extrem charakteristisch oder } \\
\text { zutreffend }\end{array}$ & 5 \\
\hline
\end{tabular}

„Messtheoretischen Pragmatikern“ (vgl. Bortz \& Döring, 1995, S. 168) folgend wird in dieser Arbeit davon ausgegangen, dass die hier verwendete Ratingskala des PQS zur Beurteilung des Therapieprozesses Intervallskalenniveau aufweist, da die Verletzungen der Intervallskaleneigenschaften bei Ratingskalen nicht so gravierend sind, als dass man auf die Verwendung parametrischer Verfahren gänzlich verzichten müsste. Somit sind statistische Analysen von Ratingskalen mittels parametrischer Verfahren zulässig ${ }^{10}$.

\section{Gütekriterien des PQS}

Gemäß den Vorgaben aus anderen Studien sollte jeder Therapiemitschnitt von mindestens zwei Beurteilern eingeschätzt werden, deren Übereinstimmung bei mindestens $r=.50$ liegen sollte (Jones, 2000). Ist dies nicht der Fall, ist ein dritter Beurteiler hinzuzuziehen. Mehrere Studien mit verschiedenen Behandlungsstichproben, die das PQS benutzt hatten, zeigten eine

\footnotetext{
${ }^{10}$ Der interessierte Leser sei bzgl. der Kontroverse um das Skalenniveau von Ratingskalen und bzgl. der mathematischen Voraussetzungen parametrischer Verfahren auf Bortz und Döring (1995, S. 168) verwiesen.
} 
Methode

Psychotherapy Process Q-Sort

durchgängig zufrieden stellende Interrater-Reliabilität (Jones et al., 1988, Jones \& Windholz, 1990, Pulos \& Jones, 1987). Für zwei Beurteiler sollte sie danach zwischen .83 und .89, für drei zwischen .89 und .92 liegen. Auch in der vorliegenden Untersuchung liegt die Reliabilität im vorgegebenen Bereich (s.u.). Die durchschnittliche Item-Reliabilität ist mit .82 akzeptabel (Jones, Parke \& Pulos, 1992).

Zur Untersuchung der diskriminanten Validität des PQS beurteilten zehn erfahrene Therapeuten mit unterschiedlichem theoretischem Hintergrund Videomitschnitte von Therapiesitzungen mit dem PQS (Pulos \& Jones, 1987). Diese Videomitschnitte zeigten drei unterschiedliche Therapieansätze, mit denen jeweils der gleiche Patient von jeweils einem sehr bekannten Vertreter der entsprechenden Therapierichtung (Albert Ellis, Fritz Perls, Carl Rogers) behandelt wurde. 52 der 100 Items konnten die rational-emotive von der Gestalttherapie unterscheiden, 47 die rational-emotive von der klienten-zentrierten und 38 die klienten-zentrierte von der Gestalttherapie. Die jeweils zehn charakteristischsten und uncharakteristischen Items für jeden der drei Therapieansätze wurden dann fünf weiteren Therapeuten mit der Aufgabe, diese 60 Items den drei Therapierichtungen zuzuordnen, vorgelegt. Diese vollständig gelungene „Kreuzvalidierung“ zeigt, dass das PQS nicht nur zwischen verschiedenen Therapieansätzen unterscheiden, sondern dass es außerdem auch das Typische eines bestimmten Therapieansatzes erfassen kann.

In einer Faktorenanalyse, die 70 Behandlungen, 130 einzelne Therapiestunden und 380 Qsorts einschloss, konnte keine eindeutige Faktorstruktur gefunden werden. Dies ist vom Autor des PQS beabsichtigt.

\section{Berechnung der Beobachterübereinstimmung}

Als Maß zur Berechnung der Beobachterübereinstimmung bei intervallskalierten Ratingskalen eignet sich die Intraklassenkorrelation (ICC; vgl. Wirtz \& Caspar, 2002; Shrout \& Fleiss, 1979; Greve \& Wentura, 1991). Die ICC ist der Produkt-Moment Korrelation immer dann vorzuziehen, wenn die Stichprobe klein ist und in wechselnden Dyaden beobachtet wurde. Die ICC basiert auf einem varianzanalytischen Ansatz, bei dem zwischen der Varianz zwischen den Beobachtungsfällen (systematische Varianz) und der Varianz innerhalb der Beobachtungsfälle, die auf eine mangelhafte Beobachterübereinstimmung hindeutet (Fehlervarianz), unterschieden wird. Ist der Unterschied zwischen den Beobachtungsfällen relativ groß bei gleichzeitig geringer Varianz innerhalb der Beobachtungsfälle, ist die ICC groß (nahe 1), d.h. die Beobachtung kann als reliabel eingestuft werden. 


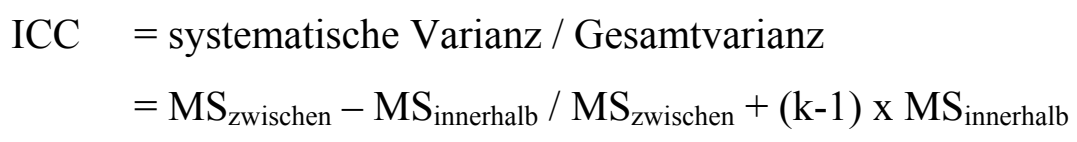

Für die vorliegende Untersuchung ist entscheidend, dass die Rater in ihren Urteilen nicht nur relativ, sondern absolut übereinstimmen sollten (,unjustierte Reliabilität“). Dies bedeutet, dass die Gesamtstichprobe raterunabhängig als Referenzstichprobe und Unterschiede zwischen den Mittelwerten der Rater als Fehler verstanden werden. Das Modell der unjustierten ICC lautet: $\mathrm{x}_{\mathrm{j}}=\mathrm{x}_{\mathrm{i}}$, weil a und $\mathrm{b}$ gleich 1 gesetzt werden, da die Varianzen und die Mittelwerte gleich sind (durch die Vorgaben des forced Q-sort). In einem solchen Fall allerdings ist die unjustierte ICC gleich der Produkt-Moment-Korrelation r. Letztere ist ein Spezialfall der ICC, wenn bei der Bestimmung des Zusammenhangs der Beurteilungen zwischen zwei Ratern deren Mittelwerte und Varianzen identisch sind (vgl. Wirtz \& Caspar, 2002).

Des Weiteren ist zwischen dem einfaktoriellen und dem zweifaktoriellen Modell der ICC zu unterscheiden. Im einfaktoriellen Modell werden die Varianzanteile, die nicht auf den Unterschied zwischen den wahren Werten der zu beurteilenden Objekte zurückzuführen sind, als Teil der Fehlervarianz verstanden. Es wird eine Varianzhomogenität sowie eine annähernde Normalverteilung der Daten verlangt. Beides ist im vorliegenden Fall erfüllt. Dieses Modell gilt auch dann, wenn die Personen von verschiedenen Ratergruppen oder von unterschiedlich vielen Ratern beurteilt werden, wie es in dieser Studie der Fall ist (Shrout \& Fleiss, 1979). Der Raterfaktor kann hier als „,random“ betrachtet werden, was bedeutet, dass die Rater repräsentativ für all diejenigen Rater sind, für die die Reliabilitätsaussage Gültigkeit haben soll.

Hill und Lambert (2004, S. 90) geben an, dass ein ICC-Wert ab .70 als gute Übereinstimmung für die Therapieprozess- und Outcome-Forschung verstanden werden kann.

\section{Ablauf der Untersuchung: Ratertraining und Durchführung der Ratings}

Für eine adäquate Anwendung des PQS reichen nach Jones et al. (1993) klinische Vorerfahrung und ein kurzes Training aus. Jones et al. (1993) schlagen vor, für ein solches Training mehrere Beurteiler, die die gleichen Sitzungen unabhängig voneinander beurteilen, einzubeziehen. Die getrennt voneinander erhobenen Einschätzungen der Sitzungen sollen im Hinblick auf Verständnisprobleme und Beurteilungsunterschiede diskutiert werden. Auch für die vorliegende Untersuchung wurde zunächst ein Ratertraining auf Basis der Manuale von 
Jones (2000) bzw. Albani et al. (2000) durchgeführt. Das Training bestand aus insgesamt 18 Treffen der Rater und über 80 Stunden Übungszeit. Erst danach wurden die ausgewählten Sitzungen der im Rahmen des GAS-Projekts behandelten Patienten mit dem PQS beurteilt.

Vor Beginn des ersten gemeinsamen Übungs-Treffens lasen sich die Rater jeweils das deutsche Manual des PQS (vgl. Albani et al., 2000) durch. Anhand dessen beurteilten sie unabhängig voneinander die Transkripte von zwei Therapiesitzungen, die nicht dem GASProjekt entstammten. Anhand der individuellen Erfahrungen beim Rating wurden in den darauf folgenden Treffen das Manual, Verständnisprobleme sowie die generelle Vorgehensweise bei der Beurteilung der Sitzungen besprochen und festgelegt. Ein besonderer Schwerpunkt der Diskussion lag auf den Items, bei denen sich die Beurteilungen der Rater um mindestens zwei Skalenpunkte voneinander unterschieden hatten: diese Items wurden besonders intensiv diskutiert, um einen Konsens in der Beurteilung erzielen zu können.

Nachdem diese beiden Therapietranskripte, die aus einer psychoanalytischen Behandlung stammten, vollständig besprochen waren, beurteilten die Rater zwei weitere, nicht aus dem GAS-Projekt stammende Transkripte unabhängig voneinander; diese wurden erneut gemeinsam mit allen Ratern besprochen. Da es auch an dieser Stelle noch zu deutlichen Unterschieden in der Beurteilung der Sitzungen kam, wurde in den weiteren Besprechungen jedes der 100 Items aus dem PQS-Manual einzeln nochmals detailliert in seiner Bedeutung diskutiert. Außerdem wurden Ankerpunkte für die Beurteilung jedes Items bestimmt. Es wurde also festgelegt, was in der Sitzung geschehen muss, damit das jeweilige Item als extrem uncharakteristisch, als extrem charakteristisch oder als neutral zu beurteilen ist. Mit diesem gemeinsam erarbeiteten Standard wurden im nächsten Schritt insgesamt vier aus der GAS-Studie stammende Therapiesitzungen probeweise beurteilt, um festzustellen, ob die Rater nun mit einem vergleichbaren Verständnis der Items operierten. Bei der Auswahl dieser Probebänder wurde sorgsam darauf geachtet, dass es sich nicht um Sitzungen solcher Patienten handelte, die später als Datenbasis dieser Untersuchung dienten und dass Sitzungsbänder aus beiden Therapieansätzen zum Training herangezogen wurden. Um zu vermeiden, dass bestimmte Items aus dem PQS während des Ratings nicht aufmerksam genug beachtet wurden, wurde außerdem gemeinsam ein Protokollbogen erarbeitet (s. Anhang A). Auf diesem Protokollbogen sind ausgewählte Items aufgeführt, bei denen die Häufigkeit bestimmter Interventionen oder Verhaltensweisen zur Einschätzung zu berücksichtigen ist (z.B. „Der Patient weist Bemerkungen des Therapeuten zurück.“). So werden die Rater auch zur Berücksichtigung derjenigen Items aufgefordert, die sie andernfalls beim Rating 
möglicherweise nicht genügend berücksichtigt hätten. Schließlich erscheint es schwierig, alle 100 Items des PQS während des gesamten Beurteilungsprozesses zu erinnern. So werden durch den Protokollbogen Hinweise zur Erinnerung gegeben.

Mit diesen Standardisierungen konnte eine befriedigende Beobachterübereinstimmung, d.h. eine Interrater-Reliabilität von zwischen .84 und .86 für die Ratings, erzielt werden. Danach wurde mit dem Rating der Sitzungen aus der Untersuchungsstichprobe begonnen. Abb. 2 zeigt den Ablauf des Trainings nochmals schematisch.

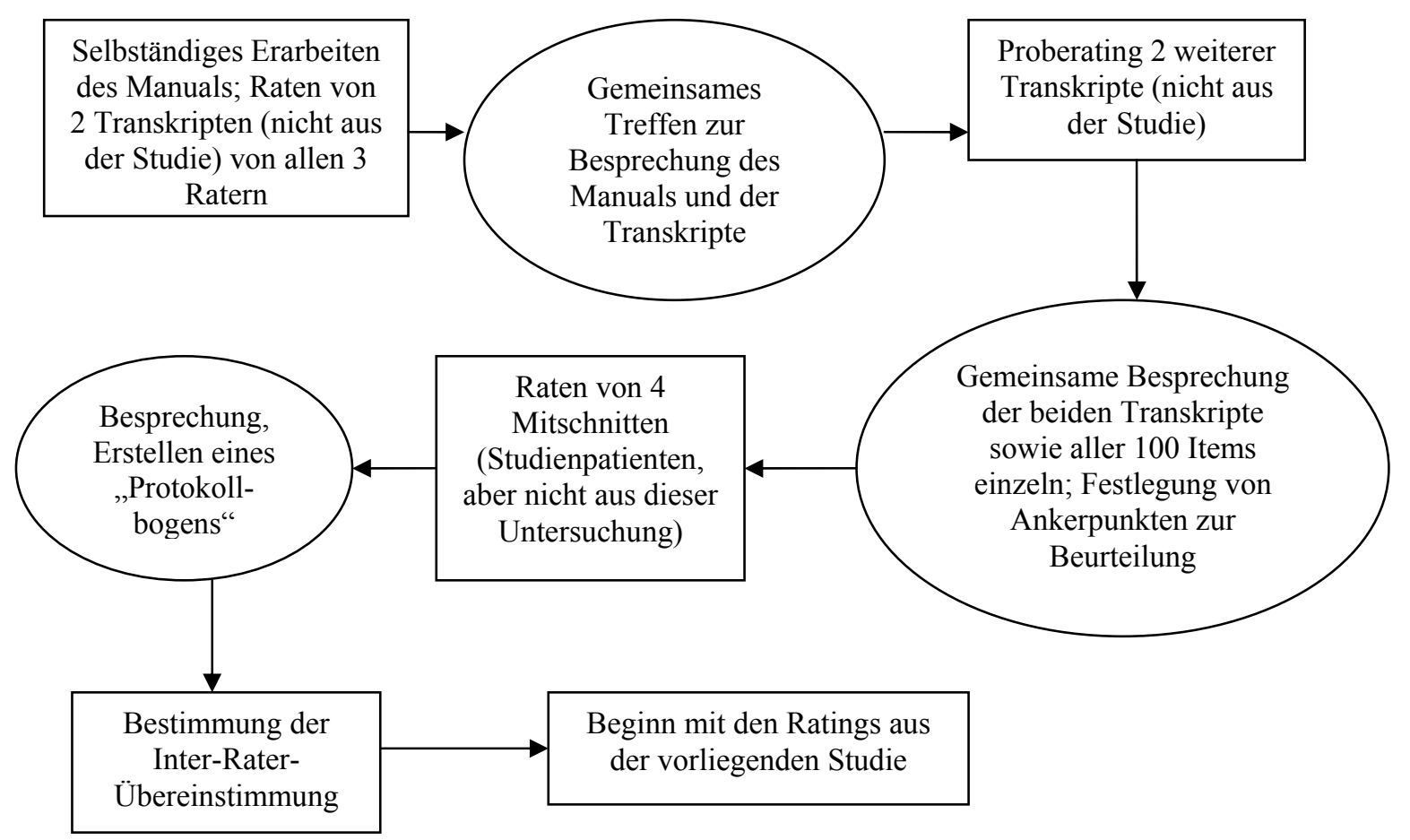

Abbildung 2: Schematische Darstellung des PQS-Trainings

Von jedem der 42 Patienten der Untersuchungsstichprobe wurden 2 Therapiesitzungen (10./18) beurteilt. Lagen diese nicht vor, wurde alternativ die nächste darauf folgende und zur Verfügung stehende Sitzung ausgewählt. Dies war bei Sitzung 10 bei drei Patienten der Fall. Zweimal wurde hier auf Sitzung 11 und einmal auf Sitzung 12 zurückgegriffen.

Jede der insgesamt 84 Sitzungen ( $N=42$ Patienten, pro Patient zwei Sitzungen) wurde von mindestens 2 von insgesamt 3 Ratern beurteilt. Die Auswahl der Reihenfolge, in der die Sitzungen beurteilt wurden, und die Zuteilung zu den drei verschiedenen Raterpaaren (A \& B, A \& C, B \& C) erfolgte randomisiert. Dazu wurden Blöcke aus anfangs sechs (z.B. Sitzung 265, 251, 27, 2, 217 und 52; wobei A \& B Sitzung 265 und 2, A \& C 27 und 52 und B \& C 


\section{Methode \\ Psychotherapy Process Q-Sort}

251 und 217 beurteilten), später zwölf zufällig ausgewählten Sitzungen (per Losverfahren) gebildet. Damit liegt ein randomisiertes balanciertes unvollständiges Block-Design vor. Die Rater wussten zum Zeitpunkt ihrer Beurteilungen weder mit welchem Therapieansatz der entsprechende Patient behandelt wurde noch welche Sitzungsnummer sie beurteilten. Folgendes Vorgehen für die Beurteilung der einzelnen Therapiesitzungen wurde vereinbart:

1. Anhören der gesamten Sitzung unter Zuhilfenahme des erarbeiteten Protokollbogens (Anhang A).

2. Durchsehen des ausgefüllten Protokollbogens und sonstiger Aufzeichnungen.

3. Einordnung der 100 Items, die durch Mischen in eine zufällige Reihenfolge gebracht wurden (um einem Reihenfolgeneffekt vorzubeugen) zunächst in 5 Kategorien (von sehr uncharakteristisch über uncharakteristisch, neutral und charakteristisch bis hin zu sehr charakteristisch), ohne dabei auf die Häufigkeiten, mit der die fünf Kategorien belegt werden, zu achten.

4. Sortieren der Items nach den Häufigkeitsvorgaben.

Es gingen die gemittelten Werte von mindestens zwei Ratern in die weiteren Analysen ein (vgl. Bortz \& Döring, 1995). Lag die Übereinstimmung zwischen dem jeweiligen Raterpaar bezogen auf die Q-Sorts eines Therapiemitschnitts unter $r=.75$, wurde die entsprechende Sitzung zusätzlich noch von einem dritten Rater beurteilt. Da die Übereinstimmung der Ratings bei drei Ratern in diesem Fall immer höher ist als bei zwei Ratern (vgl. Wirtz \& Caspar, 2002), wurden in einem solchen Fall immer die mittleren Werte aus den drei Ratings verwendet. Dies war bei fünf Sitzungen der Fall.

Um einem „observer-drift“, d.h. einer allmählichen Veränderung des Beurteilungsstandards der Rater, entgegenzuwirken, wurden folgende Maßnahmen getroffen: Nach jedem abgeschlossenen Block von Sitzungen wurde die Beobachterübereinstimmung berechnet. Danach fanden Rücksprachen der Rater untereinander (telefonisch oder face to face) statt, um Abweichungen in den Einschätzungen zu besprechen.

Die mittlere Intraklassenkorrelation über alle 84 Sitzungen dieser Untersuchung liegt bei .860 mit einem Range von .760 - .928 $($ Spannweite $=.168)$. Zur Bestimmung der durchschnittlichen Intraklassenkorrelationen werden die einzelnen ICCs zunächst in Fishers z-Werte transformiert (vgl. Bortz \& Döring, 1993). Diese werden dann gemittelt und wieder in Korrelationen rücktransformiert. Die Ergebnisse zur Beobachter-Übereinstimmung getrennt nach psychodynamischen und kognitiv-behavioralen Therapien zeigt Tabelle 7. 
Methode

Psychotherapy Process Q-Sort

Tabelle 7: Werte der Intraklassenkorrelation getrennt nach psychodynamischen und kognitivbehavioralen Behandlungssitzungen.

\begin{tabular}{|l|l|l|}
\hline Merkmal & $\begin{array}{l}\text { Kognitiv-behaviorale } \\
\text { Therapiegruppe }\end{array}$ & $\begin{array}{l}\text { Psychodynamische } \\
\text { Therapiegruppe }\end{array}$ \\
\hline Anzahl Sitzungen & $\mathrm{n}=46$ & $\mathrm{n}=38$ \\
\hline Mittelwert & .868 & .850 \\
\hline Spannweite & .155 & .156 \\
\hline Minimum & .773 & .760 \\
\hline Maximum & .928 & .916 \\
\hline
\end{tabular}

Die mittleren Werte der ICCs für die psychodynamische und die kognitiv-behaviorale Therapie liegen deutlich über den Mindestanforderungen für Beobachtermaße in der Therapieprozessforschung (.70).

Die reine Ratingphase dauerte vom Dezember 2006 bis zum Februar 2007 an. Die Auswertung eines jeden Transkriptes selbst dauerte zwischen zwei und vier Stunden.

\section{Rater der Therapiesitzungen}

Die PQS-Ratings der Therapiestunden wurden von drei verschiedenen Ratern (alle drei weiblich) durchgeführt. Rater A (zum Zeitpunkt der Ratings 26 Jahre) und Rater B (zum Zeitpunkt der Ratings 27 Jahre) waren Mitarbeiter der Abteilung Psychosomatik des Universitäts-Klinikums Göttingen mit einem Diplom in Psychologie. Rater A ist die Autorin dieser Arbeit. Rater C (zum Zeitpunkt der Untersuchung 26 Jahre) war eine Psychologiestudentin des Psychologischen Instituts der Universität Göttingen, die im Rahmen dieser Studie ihre Diplomarbeit verfasst.

Zum Zeitpunkt der Ratings standen die konkreten und detaillierten Forschungshypothesen noch nicht fest, um Beobachtereffekte zu vermeiden (vgl. Bortz \& Döring, 1995, Hill \& Lambert, 2004). Auch wurden den Ratern keine Informationen über die Patienten, die Therapeuten oder die Therapien an sich zur Verfügung gestellt. 


\section{Hypothesen und Fragestellungen und statistische Verfahren zu ihrer}

\section{Auswertung}

Vor der Auswertung der Fragestellungen wird zunächst überprüft, ob sich die beiden mittleren Intraklassenkorrelationen für die Interrater-Reliabilität der Q-Sorts in der psychodynamisch und der kognitiv-behavioral orientierten Therapie signifikant voneinander unterscheiden. Dies geschieht mittels eines z-Tests für unabhängige Stichproben.

Zur Auswertung der mit dem PQS erhobenen Daten werden, dem Vorgehen von Jones und Kollegen folgend, die zehn charakteristischsten und die zehn uncharakteristischsten Items des PQS für die entsprechende (Teil-)Stichprobe (also z.B. für alle kognitiv-behavioralen oder psychodynamischen Sitzungen) ermittelt. Dazu werden die über die entsprechenden Patienten bzw. Sitzungen und Rater gemittelten Ratings der 100 Items des PQS in eine Rangreihe absteigend geordnet. Diese insgesamt 20 Items sollen eine allgemeine Beschreibung des Verhaltens der Patienten, der Therapeuten sowie von ihrer Interaktion liefern.

\section{Hypothese 1: Die 10. und die 18. Sitzung unterscheiden sich bezogen auf die gesamte} Stichprobe und getrennt nach den beiden Therapieansätzen weder in Bezug auf das Verhalten oder den Zustand des Patienten noch auf die Interaktion zwischen Patient und Therapeut oder die therapeutischen Interventionen bedeutsam voneinander.

Der Vergleich zwischen 10. und 18. Sitzung wird in zwei Schritten durchgeführt: zunächst wird jeweils für die gesamte Stichprobe sowie für die psychodynamisch und kognitivbehavioral orientierten Therapiesitzungen getrennt eine Rangreihung zur Bestimmung der zehn charakteristischsten und der zehn uncharakteristischsten Items vorgenommen. Dann wird für jedes einzelne Item aus dem PQS ein t-Test für abhängige Stichproben zum Vergleich der entsprechenden Mittelwerte der 10. und der 18. Sitzung durchgeführt. Durch die große Anzahl an t-Tests, die hier durchzuführen sind, steigt das Risiko eines Fehlers 1. Art, d.h. Unterschiede in der Stichprobe zu finden, obwohl sie in der Population nicht existieren. Die in solchen Fällen sonst angewendete Bonferroni-Adjustierung für multiple Vergleiche wird hier nicht durchgeführt, da damit die Power der Tests sinkt. Stattdessen wird in der vorliegenden Untersuchung das Signifikanzniveau auf $\alpha=.01$ festgelegt, so wie es der Autor des PQS selbst vorgeschlagen hatte (vgl. Jones et al., 1993). Danach sind Fehler der 1. Art deshalb vertretbar, weil zunächst gefundene Effekte in weiteren Untersuchungen überprüft werden können. Fehler der 2. Art hingegen (d.h. Effekte sind zwar in der Population 
vorhanden, konnten aber in der Stichprobe nicht gefunden werden) würden nicht entdeckt werden, da keine weiteren Untersuchungen stattfänden (vgl. Jones et al., 1993).

Hypothese 2: Die Therapieprozesse psychodynamisch und kognitiv-behavioral behandelter Patienten, erfasst mit Hilfe des PQS, unterscheiden sich in Bezug auf therapeutische Techniken und Haltungen bedeutsam voneinander. In Bezug auf patientenseitige Merkmale (Haltung und emotionaler Zustand des Patienten) sowie auf die Interaktion zwischen Patient und Therapeut in der Stunde unterscheiden sich psychodynamisch und kognitiv-behavioral orientierte Therapiesitzungen jedoch nicht bedeutsam voneinander.

Beim Vergleich der psychodynamisch mit der kognitiv-behavioral behandelten Stichprobe über alle 100 Items des PQS wird die gleiche Vorgehensweise wie zum Vergleich der 10. und der 18. Sitzung gewählt. Es wird ein t-Test für unabhängige Stichproben gerechnet, um signifikante Differenzen zwischen einzelnen Items herausfinden zu können. Das Signifikanzniveau wird auch hier aus den zuvor aufgeführten Gründen auf $\alpha=.01$ festgelegt. Dabei ist zu beachten, dass sich einzelne Items als charakteristischer für die eine oder die andere Therapierichtung erweisen könnten, obgleich sie möglicherweise für beide Therapieformen in den charakteristischen oder uncharakteristischen Bereich eingeordnet werden. Dementsprechend sind diese Aussagen als relative Aussagen zu betrachten.

Außerdem ist zu bedenken, dass ein Item, das als uncharakteristisch eingeschätzt wurde, nicht als unwichtig für diese Therapieform missverstanden werden darf, sondern dass es als bedeutsam in seiner Abwesenheit eingeschätzt wurde. Im Anschluss an die t-Tests werden Chi-Quadrat-Tests berechnet, um Aussagen darüber treffen zu können, ob sich die Häufigkeiten zwischen signifikanten und nicht signifikanten Itemdifferenzen in Bezug auf die drei Kategorien an Items des PQS (emotionaler Zustand des Patienten, Interaktion zwischen Patient und Therapeut, therapeutische Interventionen) signifikant voneinander unterscheiden.

Hypothese 3: Die Orientierung am zugehörigen Prototyp ist signifikant größer als die zum gegensätzlichen Prototyp.

3a. Die kognitiv-behaviorale Therapie ähnelt dem kognitiv-behavioralen Prototyp signifikant stärker als dem psychodynamischen Prototyp.

\section{3b. Die psychodynamische Psychotherapie ähnelt dem psychodynamischen Prototyp} signifikant stärker als dem kognitiv-behavioralen Prototyp.

Zur Überprüfung des Zusammenhangs der hier implementierten Therapien mit den von Ablon und Jones (1998) erstellten Prototypen wird das Q-Sort jedes Patienten mit jeweils 
beiden Prototypen (kognitiv-behavioral und psychodynamisch) korreliert. Das Q-Sort eines Patienten entsteht aus den über alle Rater und Therapiesitzungen (10. und 18. Sitzung) gemittelten Ratings für jedes der 100 Items aus dem PQS. Das Rating eines Items wird dann mit dem entsprechenden Faktorscore des gleichen Items für beide Prototypen getrennt korreliert. Besteht zwischen zwei Merkmalen in der Population ein Zusammenhang, erhält man für (theoretisch unendlich) viele Stichproben eine linkssteile bzw. rechtssteile Korrelationsverteilung. Mit Hilfe der „r in z Transformation“ von Fisher werden diese Korrelationen in z-Werte umgewandelt, die annähernd normalverteilt sind (vgl. Bortz \& Döring, 1993). Diese geben dann an, wie stark die PQS-Beurteilung einer Therapiestunde mit dem Prototyp korreliert ist. Für jeden Patienten entstehen so zwei Korrelationen in Form von z-Werten: zum einen ein Wert für den Zusammenhang mit dem psychodynamischen und zum anderen ein Wert für den Zusammenhang mit dem kognitiv-behavioralen Prototyp. Diese zWerte werden über alle Patienten derselben Therapierichtung gemittelt, sodass der entstehende mittlere z-Wert Aufschluss darüber gibt, wie stark sich die durchgeführte Behandlung an dem prototypischen Behandlungsprozess, so wie er von entsprechenden Experten ihrer Therapierichtung beschrieben wird, orientiert hat. Dieser mittlere z-Wert gilt als Maß für die Orientierung am jeweiligen Therapiekonzept. Im Anschluss werden z-Tests für unabhängige Stichproben (vgl. Bortz \& Döring, 1993, S. 203) über diese mittleren Korrelationen mit den Prototypen (in z-Werten) gerechnet, um jeweils für einen der beiden Prototypen feststellen zu können, ob einer der beiden Therapieansätze sich signifikant mehr daran orientiert. Des Weiteren werden z-Tests für abhängige Stichproben (vgl. Bortz \& Döring, 1993, S. 204f) berechnet, um feststellen zu können, ob sich die Korrelationen mit den beiden Prototypen innerhalb eines Behandlungsarms signifikant voneinander unterscheiden. Das Signifikanzniveau wird hier, gängigen Konventionen folgend, auf $\alpha=.05$ festgelegt.

Fragestellung A: Gibt es einen bedeutsamen Zusammenhang zwischen der Stärke der Orientierung am zugehörigen Prototyp und dem Therapieoutcome? Übt die Variable „Festhalten am eigenen Prototyp“ generell einen bedeutsamen Einfluss auf das Therapieergebnis aus?

Um zu prüfen, ob die Aspekte, die nach der jeweiligen Therapietheorie für die Veränderungen im Patienten verantwortlich sein sollen (anhand der Prototypen), eine prädiktive Funktion für das Outcome haben, werden die zuvor berechneten Zusammenhänge zwischen den Prototypen und den hier implementierten Therapien (z-Wert pro Patient und pro Therapiearm) mit Outcome-Maßen korreliert. Als Outcome-Maße werden die Prä-Post- 
Differenzen des BAI, des PSWQ, des STAI, des BDI, des IIP, der HAMA und der HAMD verwendet. Diese Korrelationen ermöglichen Aussagen darüber, ob mit der Orientierung an einem bestimmten Prototyp eine signifikante Verbesserung beim Patienten einhergeht. Darüber hinaus wird „über Kreuz“ geprüft, ob die Übereinstimmung mit dem gegenteiligen Prototyp eine Vorhersage des Therapieerfolgs erlaubt (also die Übereinstimmung der PQSRatings der psychodynamischen Therapiesitzungen mit dem kognitiv-behavioralen Prototyp und umgekehrt).

Weiterhin wird aus den beiden Variablen „Korrelation mit dem kognitiv-behavioralen Prototyp“ und „Korrelation mit dem psychodynamischen Prototyp“ eine neue Variable „Orientierung am eigenen Prototyp“ bestimmt. Dazu werden die bereits bestimmten z-Werte der Patienten unabhängig vom jeweiligen Therapiearm betrachtet: von jedem psychodynamisch behandelten Patienten wird sein z-Wert für die Korrelation mit dem psychodynamischen Prototyp und für jeden kognitiv-behavioral behandelten Patienten sein zWert für die Korrelation mit dem kognitiv-behavioralen Prototyp verwendet. So entsteht die neue Variable „Orientierung am eigenen Prototyp“ für alle 42 Patienten. Diese 42 z-Werte werden dann mit den oben genannten Outcome-Maßen korreliert. Diese Korrelationen geben, unabhängig von der jeweiligen Behandlungsgruppe, wieder, wie hoch die Übereinstimmung zwischen dem jeweils zugehörigen Prototyp und der tatsächlich durchgeführten Therapie für die gesamte Stichprobe ist. So sind Aussagen über den Einfluss der Variable „Orientierung am eigenen Prototyp“ auf das Ergebnis einer Therapie, auch unabhängig von der jeweils durchgeführten Behandlungsform, möglich. Auch hier wird das Signifikanzniveau auf $\alpha=.05$ festgelegt.

\section{Fragestellung B: Sind bestimmte Items aus dem PQS signifikante Prädiktoren des}

Therapieerfolgs? Beschreiben diese prädiktiven Items eher Merkmale des Patienten oder des Therapeuten oder ihrer Interaktion? Gibt es spezifische Prädiktoren?

Die Fragestellung, ob es unter den charakteristischsten und uncharakteristischsten Items des PQS (getrennt für die gesamte Stichprobe sowie für die beiden Behandlungsgruppen) Prädiktoren des Therapieerfolgs gibt, wird mit Hilfe von linearen und multiplen Regressionen beantwortet. Als Outcome-Maße und damit als abhängige Variable in den Regressionen dienen die Prä-Post-Differenzen des BAI, des STAI, des PSWQ, des IIP, der HAMA, des BDI und der HAMD. Zunächst werden lineare Regressionen für jeweils jedes der zehn charakteristischsten und zehn uncharakteristischsten Items der interessierenden Stichprobe 
einzeln berechnet. UV ist das entsprechende Item aus dem PQS. Im Anschluss daran sollen multiple Regressionen (Methode „Einschluss“) berechnet werden. In diese gehen als UVs all die Items aus dem PQS ein, die sich in den vorangegangenen linearen Regressionen als signifikante Prädiktoren des Therapieoutcomes für ein entsprechendes Outcome-Maß erwiesen haben. Das Signifikanzniveau wird hier wegen der multiplen Tests erneut auf $\alpha=$ .01 festgelegt.

Auf eine multiple Regression, in die alle 100 Items aus dem PQS gleichzeitig eingehen, wird verzichtet, weil das Verhältnis zwischen Anzahl an Prädiktoren (i=100) und Größe der Stichprobe $(n=42)$ nicht den Anforderungen entspricht (vgl. Field, S. 172ff). 


\section{Ergebnisse}

\section{Vergleich der Intraklassenkorrelation zwischen den beiden}

\section{Behandlungsgruppen}

Ein z-Test für unabhängige Stichproben zum Vergleich der durchschnittlichen Intraklassenkorrelation der PQS-Ratings zwischen den psychodynamischen und den kognitivbehavioralen Therapiesitzungen zeigt keinen signifikanten Unterschied (ICC $=.87$ für die kognitiv-behaviorale Therapie und ICC $=.85$ für die psychodynamische Therapie; $z=0,30, p$ $=.38)$.

\section{Vergleich der PQS-Ratings für die 10. und 18. Sitzung: Überprüfung der Hypothese 1}

Zum Vergleich der PQS-Ratings der 10. und 18. Sitzung wurden zunächst die Mittelwerte aller 100 PQS-Items für die gesamte Stichprobe und für beide Therapieansätze getrennt berechnet. Diese wurden dann in eine Rangfolge gebracht. Wenn sich in dieser so erstellten Rangordnung nicht bei beiden Messzeitpunkten die gleichen 10 charakteristischsten und uncharakteristischsten Items ergaben (z.B. stimmten in beiden Rangordnungen von den zehn charakteristischsten Items nur acht überein), wurden für all diejenigen Items, die nur in einer der beiden Rangordnungen zu den charakteristischsten bzw. uncharakteristischsten zählten (also bei Sitzung 10 z.B. Q 5 und Q 12 und bei Sitzung 18 Q 89 und Q 96), ihre Mittelwerte aus den PQS-Ratings der beiden Messzeitpunkte bestimmt (also hier die Mittelwerte der PQSRatings aus den Sitzungen 10 und 18 für Item Q 5, Q 12, Q 89 und Q 96). Es wurden dann diejenigen PQS-Items in die Rangordnung der 10 charakteristischsten bzw.

uncharakteristischsten Items aufgenommen, die die höchsten bzw. niedrigsten Mittelwerte aufwiesen (z.B. wurden hier in die Rangordnung der zehn charakteristischsten Items bei beiden Messzeitpunkten die Items Q 5 und Q 89 aufgenommen, weil diese die höchsten Mittelwerte von den genannten vier Items zeigten). So wurde eine gemeinsame Liste der zehn charakteristischsten bzw. uncharakteristischsten Items auch über beide Messzeitpunkte hinweg erstellt. Das gleiche Vorgehen wurde auch für die nach den beiden Therapiearmen getrennte Überprüfung der Unterschiede zwischen den beiden Messzeitpunkten durchgeführt. Die zehn Items mit den höchsten Mittelwerten und die zehn mit den niedrigsten Mittelwerten stellen so die jeweils zehn am meisten und am wenigsten charakteristischen PQS-Items der jeweiligen Stichprobe dar. 
Vergleich über die gesamte Stichprobe $(N=42)$

Tabelle 8 zeigt die zehn charakteristischsten Items für die 10. und 18. Sitzung und ihre Rangordnung zu den beiden untersuchten Messzeitpunkten. Die Mittelwerte der PQS-Ratings auf der Skala von 1 bis 9 liegen zwischen 8,56 und 6,77 (vgl. Anhang B, Tab. 1 zu den Mittelwerten und Standardabweichungen der einzelnen Items).

Tabelle 8: 10 charakteristischste Items der gesamten Stichprobe, ihre Rangordnung und Bedeutung.

\begin{tabular}{|l|l|l|l|}
\hline Code & $\begin{array}{l}\text { Rang in der } \\
\text { 10. Sitzung }\end{array}$ & $\begin{array}{l}\text { Rang in der } \\
\text { 18. Sitzung }\end{array}$ & Bedeutung des Items \\
\hline Q 3 & 1 & 1 & $\begin{array}{l}\text { Die Äußerungen des Therapeuten zielen darauf ab, dem } \\
\text { Patienten das Erzählen zu erleichtern. }\end{array}$ \\
\hline Q 69 & 2 & 2 & $\begin{array}{l}\text { Die aktuelle oder kurz zurückliegende Lebenssituation des } \\
\text { Patienten wird besprochen. }\end{array}$ \\
\hline Q 23 & 3 & 5 & Das Gespräch hat einen spezifischen Fokus. \\
\hline Q 65 & 4 & 3 & $\begin{array}{l}\text { Der Therapeut klarifiziert, formuliert neu oder wiederholt } \\
\text { mit anderen Worten, was der Patient sagte. }\end{array}$ \\
\hline Q 88 & 5 & 6 & $\begin{array}{l}\text { Der Patient bringt relevante Themen und Material zur } \\
\text { Sprache. }\end{array}$ \\
\hline Q 80 & 6 & 4 & $\begin{array}{l}\text { Der Therapeut bietet eine Erfahrung oder ein Ereignis aus } \\
\text { einer anderen Perspektive an. }\end{array}$ \\
\hline Q 46 & 7 & 7 & $\begin{array}{l}\text { Der Kommunikationsstil des Therapeuten ist klar und } \\
\text { verständlich. }\end{array}$ \\
\hline Q 30 & 8 & 8 & $\begin{array}{l}\text { Die Diskussion ist um kognitive Themen zentriert, d.h. } \\
\text { Gedanken und Überzeugungen. }\end{array}$ \\
\hline Q 54 & 9 & 10 & $\begin{array}{l}\text { Der Patient ist in seiner Ausdrucksweise klar und } \\
\text { strukturiert. }\end{array}$ \\
\hline Q 35 & 10 & 9 & Das Selbstbild ist ein Fokus des Gesprächs. \\
\hline
\end{tabular}

Anm: die fett gedruckten Items sind die, die nicht von Beginn an in beiden Teilstichproben (10. und 18. Sitzung) zu den jeweils 10 charakteristischsten bzw. uncharakteristischsten gehörten und die erst über die im Text beschriebene Vorgehensweise bestimmt wurden.

In den zehn uncharakteristischsten Items stimmen die 10. und 18. Sitzung vollkommen überein (vgl. Tab. 9). Die Mittelwerte der PQS-Ratings auf der Skala von 1 bis 9 liegen zwischen 1,08 und 2,65 (vgl. Anhang B, Tab. 1).

Zur Analyse der Unterschiede zwischen 10. und 18. Sitzung wurden die PQS-Ratings der 10. Sitzung mit denen der 18. Sitzung in zweiseitigen t-Tests für abhängige Stichproben für jedes Item des PQS einzeln getestet. Das Signifikanzlevel wurde auf $\alpha=.01$ festgelegt. Die Ergebnisse der t-Tests zeigen, dass sich die 10. und 18. Sitzung in keinem einzigen Item des PQS signifikant voneinander unterscheiden (vgl. Anhang B, Tab. 2). 
Tabelle 9: 10 uncharakteristischste Items der gesamten Stichprobe, ihre Rangordnung und Bedeutung.

\begin{tabular}{|c|c|c|c|}
\hline Code & $\begin{array}{l}\text { Rang in der } \\
\text { 10. Sitzung }\end{array}$ & $\begin{array}{l}\text { Rang in der } \\
\text { 18. Sitzung }\end{array}$ & Bedeutung des Items \\
\hline Q 67 & 91 & 92 & $\begin{array}{l}\text { Der Therapeut interpretiert verdrängte oder unbewusste } \\
\text { Wünsche, Gefühle oder Überzeugungen. }\end{array}$ \\
\hline Q 33 & 92 & 94 & $\begin{array}{l}\text { Der Patient spricht von Nähe oder vom Wunsch nach Nähe } \\
\text { oder davon, jemanden zu brauchen. }\end{array}$ \\
\hline Q 42 & 93 & 91 & $\begin{array}{l}\text { Der Patient weist die Bemerkungen und Beobachtungen des } \\
\text { Therapeuten zurück (vs. er nimmt sie an). }\end{array}$ \\
\hline Q 29 & 94 & 93 & $\begin{array}{l}\text { Der Patient spricht davon, dass er Trennung von oder Distanz } \\
\text { will. }\end{array}$ \\
\hline Q 36 & 95 & 95 & $\begin{array}{l}\text { Der Therapeut weist auf Abwehrmechanismen des Patienten } \\
\text { hin, z.B. Ungeschehenmachen, Verleugnung. }\end{array}$ \\
\hline Q 49 & 96 & 96 & $\begin{array}{l}\text { Der Patient erlebt dem Therapeuten gegenüber ambivalente } \\
\text { oder widersprüchliche Gefühle. }\end{array}$ \\
\hline Q 90 & 97 & 98 & Träume und Phantasien werden besprochen. \\
\hline Q 91 & 98 & 97 & $\begin{array}{l}\text { Erinnerungen oder Rekonstruktionen der (frühen und späten) } \\
\text { Kindheit sind Gegenstand des Gesprächs. }\end{array}$ \\
\hline Q 2 & 99 & 99 & $\begin{array}{l}\text { Der Therapeut lenkt die Aufmerksamkeit auf das nonverbale } \\
\text { Verhalten des Patienten, z.B. auf die Körperhaltung oder } \\
\text { Gesten. }\end{array}$ \\
\hline Q 11 & 100 & 100 & Sexuelle Gefühle und Erfahrungen werden besprochen. \\
\hline
\end{tabular}

Vergleich über die psychodynamisch behandelte Teilstichprobe $(n=19)$

$\mathrm{Zu}$ den zehn charakteristischsten Items der psychodynamisch behandelten Stichprobe gehören Item Q 3, Q 23, Q 35, Q 46, Q 54, Q 69, Q 80 und Q 88 (Bedeutung der Items vgl. Tab. 1). Daneben gehört weiterhin Item Q 6 („Der Therapeut ist einfühlsam und empathisch.“) zu den zehn charakteristischsten Items. Die Mittelwerte der PQS-Ratings liegen zwischen 6,63 und 8,53 (vgl. Anhang B, Tab. 3).

$\mathrm{Zu}$ den zehn uncharakteristischsten Items der psychodynamisch behandelten Stichprobe zählt neben den Items Q 2, Q 11, Q 29, Q 33, Q 36, Q 42, Q 49, Q 90 und Q 91 (Bedeutung der Items vgl. Tab. 2) auch Item Q 38 („Es werden spezifische Aktivitäten oder Aufgaben besprochen, die der Patient außerhalb der Sitzungen in Angriff nehmen soll.“). Die Mittelwerte der PQS-Ratings liegen zwischen 1,13 und 2,82 (vgl. Anhang B, Tab. 3).

Im Vergleich über zweiseitige t-Tests für abhängige Stichproben für jedes einzelne Item des PQS zwischen der 10. und 18. psychodynamisch orientierten Behandlungssitzung zeigte sich bei keinem einzigen Item ein signifikanter Unterschied (vgl. Anhang B, Tab. 4).

Vergleich über die kognitiv-behavioral behandelte Teilstichprobe $(n=23)$

$\mathrm{Zu}$ den zehn charakteristischsten Items der kognitiv-behavioral behandelten Stichprobe gehören Item Q 3, Q 23, Q 30, Q 46, Q 65, Q 69, Q 80 und Q 88 (Bedeutung der Items vgl. 
Tab. 1). Daneben gehören weiterhin die Items Q 45 („Der Therapeut nimmt eine supportive Haltung ein.“) und Q 72 (,Der Patient versteht das Wesen der Therapie und was von ihm erwartet wird.“) dazu. Die Mittelwerte der PQS-Ratings liegen zwischen 7,05 und 8,73 (vgl. Anhang B, Tab. 5).

Zu den zehn uncharakteristischsten Items der kognitiv-behavioral behandelten Stichprobe zählen die Items Q 2, Q 11, Q 29, Q 33, Q 36, Q 42, Q 49, Q 67, Q 90 und Q 91 (Bedeutung der Items vgl. Tab. 2). Die Mittelwerte der PQS-Ratings liegen zwischen 1,04 und 2,50 (vgl. Anhang B, Tab. 5).

Auch im Vergleich per zweiseitigen t-Tests für abhängige Stichproben für jedes einzelne Item des PQS zwischen der 10. und 18. kognitiv-behavioralen Behandlungssitzung zeigte sich bei keinem einzigen Item ein signifikanter Unterschied (vgl. Anhang B, Tab. 6).

In keinem einzigen der durchgeführten Vergleiche (10. vs. 18; 10. psychodynamisch orientierte vs. 18. psychodynamisch orientierte; 10. kognitiv-behavioral orientierte vs. 18. kognitiv-behavioral orientierte Sitzung) ergaben sich bedeutsame Therapieprozessunterschiede zwischen den beiden Therapiezeitpunkten. Die Hypothese 1 konnte damit bestätigt werden.

Weil insgesamt keine Differenzen zwischen der 10. und 18. Sitzung festgestellt werden konnten, werden die PQS-Ratings der 10. und 18. Sitzung für die weiteren Berechnungen über jeden Patienten und alle Rater gemittelt, sodass im Folgenden für jeden der 42 Patienten jeweils ein über beide Messzeitpunkte und alle Rater gemitteltes Q-Sort (d.h. für jeden Patienten für jedes Item des PQS nur ein Wert) in die Analysen eingeht.

\section{Vergleich der PQS-Ratings der psychodynamisch und der kognitiv- behavioral behandelten Patienten: Überprüfung der Hypothese 2}

Zum Vergleich der psychodynamisch mit den kognitiv-behavioral orientierten Therapiesitzungen werden zunächst Rangfolgen mit den jeweils zehn am meisten und am wenigsten charakteristischen PQS-Items (über beide Messzeitpunkte gemittelt) getrennt für die psychodynamischen und die kognitiv-behavioralen Therapien erstellt.

Folgende 7 Items des PQS gehören sowohl in der psychodynamisch als auch in der kognitiv-behavioral behandelten Stichprobe zu den zehn charakteristischsten Items: Q 3, Q 23, Q 46, Q 65, Q 69, Q 80 und Q 88 (Bedeutung der Items vgl. Anhang A). In der psychodynamisch behandelten Stichprobe gehören außerdem die Items Q 6, Q 35 und Q 54 
(Bedeutung der Items vgl. Anhang A) zu den 10 charakteristischsten Items. In der kognitivbehavioral behandelten Stichprobe sind es die Items Q 30, Q 45 und Q 72 (Bedeutung der Items vgl. Anhang A). Die Mittelwerte der PQS-Ratings liegen zwischen 6,86 und 8,70 (vgl. Anhang B, Tab. 7)

Neun von zehn der uncharakteristischsten Items kommen sowohl in der kognitivbehavioral als auch in der psychodynamisch behandelten Stichprobe vor. Dies sind die Items Q 2, Q 11, Q 29, Q 33, Q 36, Q 42, Q 49, Q 90 und Q 91 (Bedeutung der Items vgl. Anhang A). Zu den zehn uncharakteristischsten Items der psychodynamisch behandelten Stichprobe gehört außerdem Item Q 38 (,Es werden spezifische Aktivitäten oder Aufgaben besprochen, die der Patient außerhalb der Sitzungen in Angriff nehmen soll.“), zu denen der kognitivbehavioral behandelten Stichprobe Item Q 67 (Bedeutung des Items vgl. Anhang A). Die Mittelwerte der PQS-Ratings liegen zwischen 1,05 und 2,81 (vgl. Anhang B, Tab. 7).

Tabelle 10: PQS-Items, die signifikant charakteristischer für die kognitiv-behaviorale Therapie sind.

\begin{tabular}{|l|l|l|l|}
\hline $\begin{array}{l}\text { PQS- } \\
\text { Code }\end{array}$ & Beschreibung des PQS-Items & $\begin{array}{l}\text { M und sd } \\
\text { für pd }\end{array}$ & $\begin{array}{l}\text { M und sd } \\
\text { für KBT }\end{array}$ \\
\hline 30 & $\begin{array}{l}\text { Die Diskussion ist um kognitive Themen zentriert, d.h. Gedanken oder } \\
\text { Überzeugungen. }\end{array}$ & $6,59(1,20)$ & $7,56^{*}(.85)$ \\
\hline 46 & Der Kommunikationsstil des Therapeuten ist klar und verständlich. & $6,93(.29)$ & $7,53^{* *}(.63)$ \\
\hline 45 & Der Therapeut nimmt eine supportive Haltung ein. & $6,38(1,06)$ & $7,30^{*}(1,05)$ \\
\hline 27 & $\begin{array}{l}\text { Der Therapeut gibt explizit Ratschläge und Anleitungen (vs. weigert sich, } \\
\text { dies zu tun, selbst wenn er dazu gedrängt wird). }\end{array}$ & $5,92(1,22)$ & $7,07^{* *}(.71)$ \\
\hline 17 & $\begin{array}{l}\text { Der Therapeut kontrolliert die Interaktion aktiv (z.B. strukturiert und/oder } \\
\text { führt neue Themen ein). }\end{array}$ & $5,61(1,32)$ & $\begin{array}{l}7,05^{* *} \\
(1,04)\end{array}$ \\
\hline 86 & $\begin{array}{l}\text { Der Therapeut ist zuversichtlich oder selbstsicher (vs. unsicher oder } \\
\text { defensiv). }\end{array}$ & $6,16(.59)$ & $6,87^{* *}(.37)$ \\
\hline 85 & $\begin{array}{l}\text { Der Therapeut ermuntert den Patienten, neue Verhaltensweisen im } \\
\text { Umgang mit anderen auszuprobieren. }\end{array}$ & $5,48(.97)$ & $6,27^{*}(.85)$ \\
\hline 66 & Der Therapeut beruhigt den Patienten. & $5,28(1,17)$ & $6,17^{*}(.87)$ \\
\hline 37 & Der Therapeut verhält sich ,lehrerhaft. & $4,75(.84)$ & $5,74^{* *}(.72)$ \\
\hline 57 & $\begin{array}{l}\text { Der Therapeut erläutert das Rational seiner Technik oder seines } \\
\text { Behandlungsansatzes. }\end{array}$ & $3,76(.93)$ & $\begin{array}{l}5,47^{* *} \\
(1,09)\end{array}$ \\
\hline 38 & $\begin{array}{l}\text { Es werden spezifische Aktivitäten oder Aufgaben besprochen, die der } \\
\text { Patient außerhalb der Sitzungen in Angriff nehmen soll. }\end{array}$ & $2,60(1,83)$ & $\begin{array}{l}5,30^{* *} \\
(1,61)\end{array}$ \\
\hline
\end{tabular}

Anm: PQS = Psychotherapy Process Q-Sort, pd = psychodynamische Therapie, KBT = kognitiv-behaviorale Therapie, $\mathrm{M}=$ Mittelwert der PQS-Ratings, sd: Standardabweichung; ${ }^{*} \mathrm{p} \leq .01, * * \mathrm{p} \leq .001$.

Im Anschluss wurde jedes der 100 Items aus dem PQS einzeln auf Unterschiede zwischen der psychodynamisch und der kognitiv-behavioral behandelten Stichprobe untersucht. Dazu wird für jedes Item ein zweiseitiger t-Test für unabhängige Stichproben gerechnet. Siebzig Items aus dem PQS differenzieren nicht signifikant zwischen der kognitiv-behavioral und der psychodynamisch behandelten Stichprobe. Die folgenden Tabellen 10 und 11 listen die 
dreißig Items auf, in denen sich die beiden Therapieformen signifikant voneinander unterscheiden.

Tabelle 11: PQS-Items, die signifikant charakteristischer für die psychodynamische Therapie sind.

\begin{tabular}{|c|c|c|c|}
\hline $\begin{array}{l}\text { PQS- } \\
\text { Code }\end{array}$ & Beschreibung des PQS-Items & $\begin{array}{l}\text { M und sd } \\
\text { für pd }\end{array}$ & $\begin{array}{l}\text { M und sd } \\
\text { für KBT }\end{array}$ \\
\hline 65 & $\begin{array}{l}\text { Der Therapeut klarifiziert, formuliert neu oder wiederholt mit anderen } \\
\text { Worten, was der Patient sagte. }\end{array}$ & $8,45^{*}(.55)$ & $7,64(1,21)$ \\
\hline 6 & Der Therapeut ist einfühlsam, auf den Patienten eingestellt, empathisch. & $7,04 *(.75)$ & $6,49(.46)$ \\
\hline 81 & $\begin{array}{l}\text { Der Therapeut betont die Gefühle des Patienten, um ihm zu helfen, diese } \\
\text { intensiver zu erleben. }\end{array}$ & $\begin{array}{l}6,85^{* *} \\
(1,11)\end{array}$ & $5,45(.91)$ \\
\hline 50 & $\begin{array}{l}\text { Der Therapeut lenkt die Aufmerksamkeit auf Gefühle, die der Patient für } \\
\text { inakzeptabel hält (z.B. Wut, Neid oder Aufregung). }\end{array}$ & $\begin{array}{l}5,80 * * \\
(.74)\end{array}$ & $5,08(.47)$ \\
\hline 12 & In der Stunde wird geschwiegen. & $\begin{array}{l}5,57 * * \\
(.99)\end{array}$ & $4,57(.64)$ \\
\hline 28 & Der Therapeut nimmt den therapeutischen Prozess richtig wahr. & $5,55^{*}(.65)$ & $5,09(.19)$ \\
\hline 98 & Die therapeutische Beziehung ist ein Fokus des Gesprächs. & $5,30 *(.76)$ & $4,71(.40)$ \\
\hline 9 & $\begin{array}{l}\text { Der Therapeut ist distanziert, unbeteiligt (vs. empfänglich und affektiv } \\
\text { involviert). }\end{array}$ & $\begin{array}{l}5,05^{* *} \\
(.58)\end{array}$ & $4,39(.49)$ \\
\hline 87 & Der Patient kontrolliert. & $\begin{array}{l}4,86^{*} \\
(1,27) \\
\end{array}$ & $3,90(1,01)$ \\
\hline 93 & Der Therapeut ist neutral. & $\begin{array}{l}4,84^{*} \\
(1,09)\end{array}$ & $3,82(.91)$ \\
\hline 83 & Der Patient ist fordernd. & $4,77 *(.57)$ & $4,25(.58)$ \\
\hline 8 & $\begin{array}{l}\text { Der Patient ist besorgt oder beunruhigt wegen seiner Abhängigkeit vom } \\
\text { Therapeuten (vs. fühlt sich wohl mit der Abhängigkeit oder will } \\
\text { Abhängigkeit) }\end{array}$ & $4,54 *(.45)$ & $4,08(.54)$ \\
\hline 58 & $\begin{array}{l}\text { Der Patient weigert sich, sich mit eigenen Gedanken, Reaktionen oder } \\
\text { Motiven, die mit seinen Problemen in Zusammenhang stehen, } \\
\text { auseinanderzusetzen. }\end{array}$ & $4,38^{*}(.80)$ & $3,75(.58)$ \\
\hline 77 & Der Therapeut ist taktlos. & $4,24 *(.45)$ & $3,86(.38)$ \\
\hline 44 & Der Patient ist wachsam und misstrauisch (vs. vertrauensvoll und sicher). & $\begin{array}{l}3,87^{* *} \\
(.92) \\
\end{array}$ & $3,01(.38)$ \\
\hline 67 & $\begin{array}{l}\text { Der Therapeut interpretiert verdrängte oder unbewusste Wünsche, } \\
\text { Gefühle oder Überzeugungen. }\end{array}$ & $\begin{array}{l}3,06^{* *} \\
(1,01)\end{array}$ & $2,23(.46)$ \\
\hline 39 & Die Beziehung hat rivalisierenden Charakter. & $\begin{array}{l}3,02 * * \\
(.26)\end{array}$ & $2,67(.29)$ \\
\hline 36 & $\begin{array}{l}\text { Der Therapeut weist auf Abwehrmechanismen des Patienten hin, z.B. } \\
\text { Ungeschehenmachen, Verleugnung. }\end{array}$ & $2,41 *(.85)$ & $1,79(.46)$ \\
\hline 49 & $\begin{array}{l}\text { Der Patient erlebt dem Therapeuten gegenüber ambivalente oder } \\
\text { widersprüchliche Gefühle. }\end{array}$ & $\begin{array}{l}2,23^{*} \\
(1,12)\end{array}$ & $1,51(.28)$ \\
\hline
\end{tabular}

Anm: PQS = Psychotherapy Process Q-Sort, $\mathrm{pd}=$ psychodynamische Therapie, $\mathrm{KBT}=$ kognitiv-behaviorale Therapie, $\mathrm{M}=$ Mittelwert der PQS-Ratings, sd: Standardabweichung; $* \mathrm{p} \leq .01,{ }^{*} \mathrm{p} \leq .001$.

Von den Items, die signifikant charakteristischer für die psychodynamisch behandelte Stichprobe sind, gehören die Items Q 6 und Q 54 zu den zehn charakteristischsten und Item Q 36 und Q 49 zu den zehn uncharakteristischsten Items der psychodynamisch behandelten Stichprobe. 
Von den Items, die signifikant charakteristischer für die kognitiv-behavioral behandelte Stichprobe sind, gehören die Items Q 30, Q 45 und Q 46 zu den zehn charakteristischsten der kognitiv-behavioral behandelten Stichprobe.

Bei sechs Items, die die Haltung oder den emotionalen Zustand des Patienten in der Therapie beschreiben, unterscheiden sich die beiden Therapiegruppen signifikant voneinander. Der Patient in der psychodynamischen Therapie kontrolliert stärker (Q 87), fordert mehr (Q 83), ist wachsamer und misstrauischer (Q 44), erlebt mehr Ambivalenz gegenüber seinem Therapeuten (Q 49), ist stärker besorgt oder beunruhigt wegen seiner Abhängigkeit vom Therapeuten (Q 8) und weigert sich deutlicher, sich mit seinen eigenen Gedanken, Reaktionen oder Motiven, die mit seinen Problemen im Zusammenhang stehen, auseinander zu setzen (Q 58).

Hinsichtlich der Interaktion zwischen Patient und Therapeut bzw. den in der Sitzung fokussierten Themen unterscheiden sich die beiden Therapiegruppen bei fünf Items signifikant voneinander. In der psychodynamisch orientierten Therapie hat die Beziehung zwischen Patient und Therapeut einen stärker rivalisierenden Charakter (Q 39) und wird mehr fokussiert (Q98) und es wird mehr geschwiegen (Q 12). In der kognitiv-behavioral orientierten Therapie werden insgesamt mehr kognitive Themen (Q 30) und spezifische Aktivitäten, die der Patient außerhalb der Therapie in Angriff nehmen soll, besprochen (Q38).

$\mathrm{Zu}$ den Interventionen, die der psychodynamisch orientierte Therapeut im Vergleich zum kognitiv-behavioral orientierten Therapeuten häufiger anwendet, gehört, dass er die Aufmerksamkeit stärker auf Gefühle, die der Patient für inakzeptabel hält, lenkt (Q 50), dass er häufiger (wenn auch insgesamt eher selten) auf Abwehrmechanismen des Patienten hinweist (Q 36), dass er häufiger verdrängtes oder unbewusstes Material interpretiert (Q 67) und dass er stärker die Gefühle des Patienten betont, damit dieser sie intensiver erleben kann (Q 81). Im Vergleich dazu gibt der kognitiv-behavioral orientierte Therapeut häufiger explizit Ratschläge und Anleitungen (Q 27), erläutert eher das Rational seiner Technik (Q 57) und ermuntert den Patienten stärker zu neuen Verhaltensweisen im Umgang mit anderen (Q 85).

Auch bzgl. der Haltung des Therapeuten gegenüber dem Patienten unterscheiden sich die beiden Therapien voneinander. Psychodynamisch orientierte Therapeuten werden als distanzierter (Q 9), neutraler (Q 93) und taktloser (Q 77), aber auch als empathischer (Q 6) beurteilt und können den therapeutischen Prozess und den emotionalen Zustand des Patienten besser einschätzen (Q 28). Kognitiv-behavioral orientierte Therapeuten kontrollieren die Interaktion aktiver (Q 17), verhalten sich „lehrerhafter“ (Q 37), sind supportiver (Q 45) und 
beruhigen den Patienten mehr (Q 66). Ihr Kommunikationsstil ist klarer und verständlicher (Q 46) und sie sind zuversichtlicher oder selbstsicherer (Q 86). Psychodynamische Interventionen sind häufiger Klarifikation, Neuformulierung oder Wiederholung von Aspekten, die der Patient angesprochen hat (Q 65).

Von insgesamt 100 Items unterscheiden sich also nur dreißig signifikant zwischen den beiden hier untersuchten Therapieansätzen. 19 von diesen spiegeln die Haltung und die Handlungen des Therapeuten wieder, 6 betreffen das Verhalten, das Erleben oder die Haltung des Patienten. Die restlichen fünf beschreiben die Art der Interaktion bzw. das Klima innerhalb dieser.

Fast die Hälfte (33) der Items, die sich nicht bedeutsam unterscheiden sind Items, die die Haltung oder den emotionalen Zustand des Patienten beschreiben. Keine signifikanten Unterschiede zeigten sich z.B. in den Items Q 13 (Lebhaftigkeit des Patienten) und Q 26 (Empfindung unangenehmer oder schmerzlicher Gefühle wie Traurigkeit (Q 94), Angst (Q 7), Scham oder Schuld (Q 71)). Auch fühlen sich die Patienten beider Therapiegruppen gleichermaßen wenig unzulänglich und unterlegen (Q 59) sowie schüchtern und verlegen (Q 61) und verhalten sich ihrem Therapeuten gegenüber recht ähnlich: sie nehmen seine Bemerkungen an (Q 42), äußern eher positive als negative Gefühle ihrem Therapeuten gegenüber (Q 1) und verhalten sich angepasst (Q 20). Ebenfalls keine Unterschiede zeigen sich darin, wie gut der Patient den Therapeuten verstehen kann (Q 5) und sich von ihm verstanden fühlt (Q 14), wie gut er das Wesen der Therapie versteht (Q 72), wie sehr er sich der therapeutischen Arbeit verpflichtet fühlt (Q 73) und wie häufig er bedeutsame Themen zur Sprache bringt (Q 88). Die Patienten beider Therapieansätze fühlen sich gleichermaßen unterstützt (Q 95), haben positive Erwartungen bezüglich der Therapie (Q 55) und gewinnen ein vergleichbares Maß an neuer Einsicht oder an Verständnis (Q 32).

Von insgesamt 39 Items im PQS, die den emotionalen Zustand oder die Haltung des Patienten in der Therapie beschreiben, können nur 6 zwischen den beiden Therapiegruppen unterscheiden. Ein Chi-Quadrat-Test zeigt, dass die Items, die nicht signifikant zwischen den beiden Therapiegruppen unterscheiden können, signifikant häufiger sind als die Items, die signifikant zwischen den beiden Gruppen unterscheiden können $\left(\chi^{2}=9,35, \mathrm{p} \leq .01\right)$. Das gleiche gilt für die Items, die die Interaktion zwischen Patient und Therapeut bzw. die in der Sitzung fokussierten Themen beschreiben. Hier erweisen sich 5 von 25 Items als signifikant. Auch hier zeigt ein Chi-Quadrat-Test einen signifikanten Unterschied in der Häufigkeit der signifikanten und nicht signifikanten Itemdifferenzen $\left(\chi^{2}=4,5, p \leq .05\right)$. Hinsichtlich der 
Items, die therapeutische Interventionen beschreiben, gibt es bei 19 von 36 Items signifikante Differenzen. Diese Häufigkeiten unterscheiden sich nicht signifikant voneinander $\left(\chi^{2}=\right.$ 0,056). Die Hypothese, dass sich Unterschiede zwischen den beiden Therapiegruppen v.a. im Hinblick auf therapeutische Interventionen (19 von 30 signifikanten Itemdifferenzen) und weniger im Hinblick auf die Haltung und den emotionalen Zustand des Patienten (33 von 70 nichtsignifikanten Itemdifferenzen) bzw. die Interaktion zwischen Therapeut und Patient zeigen (20 von 70 nichtsignifikanten Itemdifferenzen), hat sich bestätigt.

\section{4. Übereinstimmung der implementierten Therapien mit den jeweiligen Prototypen: Überprüfung der Hypothese 3}

Zum Vergleich der beiden hier durchgeführten Psychotherapien mit den von Ablon und Jones (1998) erstellten Prototypen einer kognitiv-behavioralen und einer psychodynamischen Kurzzeittherapie wurde zunächst das individuelle Q-Sort-Profil jedes der 42 Patienten aus der GAS-Studie mit jedem Faktorscore des entsprechenden Items aus der Studie von Ablon und Jones (1998; zur Bestimmung der Faktorscores vgl. Abschnitt 3.3 in Kapitel II) von jedem der beiden Prototypen korreliert. Für die weiteren statistischen Analysen wurden die Korrelationskoeffizienten nach Pearson mit Hilfe der Fisher r-zu-z-Transformation in z-Werte transformiert (vgl. Abschnitt 4 in Kapitel III). Der jeweilige z-Score spiegelt dann das Ausmaß wider, in dem die entsprechende, aus Sitzung 10 und 18 gemittelte Therapiestunde mit dem jeweiligen Prototyp korreliert. Die durchschnittlichen z-transformierten Korrelationen über alle 42 Patienten geben an, wie stark die beiden implementierten Therapieansätze insgesamt mit den jeweiligen Prototypen übereinstimmen.

Während die Q-Sorts aller 42 Patienten signifikant mit dem Prototyp einer kognitivbehavioralen Therapie korrelieren, gilt dies nur für zwei von 42 Patienten für den psychodynamischen Prototyp (vgl. Tab. 12). Die durchschnittliche Korrelation über die QSorts von allen Patienten aus der Stichprobe $(\mathrm{N}=42)$ mit dem kognitiv-behavioralen Prototyp beträgt $.546(\mathrm{sd}=.128)$, die mit dem psychodynamischen Prototyp -.060 $(\mathrm{sd}=.100)$. Diese Korrelationen unterscheiden sich signifikant voneinander $(\mathrm{z}=4,97, \mathrm{p}=.000)$. 
Tabelle 12: Korrelationen der individuellen Q-Sorts der Patienten mit den beiden Prototypen und ihre z-Werte.

\begin{tabular}{|c|c|c|c|}
\hline Patient & Therapiegruppe & $\begin{array}{l}r \text { mit dem Prototyp einer } \\
\text { KBT }\end{array}$ & $\begin{array}{l}r \text { mit dem Prototyp } \\
\text { einer pd }\end{array}$ \\
\hline 02 & $\mathrm{pd}$ & $.558^{* *}$ & -.024 \\
\hline 06 & KBT & $.662 * *$ & -.171 \\
\hline 102 & $\mathrm{pd}$ & $.359 * *$ & .062 \\
\hline 106 & KBT & $.648 * *$ & -.113 \\
\hline 109 & KBT & $.495 * *$ & -.008 \\
\hline 11 & pd & $.245^{*}$ & .173 \\
\hline 114 & KBT & $.670 * *$ & -.088 \\
\hline 115 & pd & $: 587 * *$ & .020 \\
\hline 117 & KBT & $.698 * *$ & -.084 \\
\hline 12 & KBT & $.651 * *$ & -.032 \\
\hline 122 & $\mathrm{pd}$ & $.483 * *$ & -.025 \\
\hline 124 & KBT & $.681 * *$ & -.066 \\
\hline 125 & $\mathrm{pd}$ & $.329 * *$ & -.058 \\
\hline 126 & KBT & $.571 * *$ & -.168 \\
\hline 140 & pd & $.429 * *$ & .080 \\
\hline 142 & pd & $.551 * *$ & -.128 \\
\hline 146 & KBT & $.601 * *$ & -.084 \\
\hline 158 & KBT & $.637 * *$ & -.090 \\
\hline 161 & KBT & $.558 * *$ & -.142 \\
\hline 167 & KBT & $.678 * *$ & -.109 \\
\hline 171 & KBT & $.701 * *$ & -.156 \\
\hline 175 & $\mathrm{pd}$ & $.414^{* *}$ & -.057 \\
\hline 182 & $\mathrm{pd}$ & $.581 * *$ & -.022 \\
\hline 186 & KBT & $.648 * *$ & -.182 \\
\hline 187 & $\mathrm{pd}$ & $.368 * *$ & .145 \\
\hline 191 & KBT & $.673 * *$ & -.084 \\
\hline 197 & $\mathrm{pd}$ & $.457 * *$ & -.040 \\
\hline 202 & $\mathrm{pd}$ & $.203^{*}$ & .110 \\
\hline 206 & KBT & $.596^{* *}$ & -.086 \\
\hline 208 & KBT & $.583 * *$ & -.076 \\
\hline 211 & KBT & $.679 * *$ & -.164 \\
\hline 215 & $\mathrm{pd}$ & $.531 * *$ & .130 \\
\hline 216 & $\mathrm{pd}$ & $.474 * *$ & $-.227^{*}$ \\
\hline 229 & KBT & $.645 * *$ & -.103 \\
\hline 231 & KBT & $.547 * *$ & -.186 \\
\hline 32 & $\mathrm{pd}$ & $.396 * *$ & .005 \\
\hline 41 & KBT & $.616^{* *}$ & -.117 \\
\hline 61 & KBT & $.679 * *$ & -.119 \\
\hline 67 & $\mathrm{pd}$ & $.382 * *$ & .054 \\
\hline 73 & pd & $.622 * *$ & $-.201 *$ \\
\hline 74 & KBT & $.621 * *$ & -.173 \\
\hline 96 & $\mathrm{pd}$ & $.429 * *$ & .080 \\
\hline
\end{tabular}

Anm: ${ }^{* *} \mathrm{p} \leq .01,{ }^{*} \mathrm{p} \leq .05, \mathrm{pd}$ : psychodynamische Therapie, KBT: kognitiv-behaviorale Therapie

In der Gruppe der Patienten, die mit der psychodynamischen Kurzzeittherapie behandelt wurden (vgl. Tab. 13), zeigte sich eine durchschnittliche Korrelation von $r=.004(s d=.110$, 
z-Score von .003) mit dem psychodynamischen Prototyp $(\mathrm{sd}=.111)$ und von $\mathrm{r}=.442(\mathrm{sd}=$ .114 , z-Score von .483) mit dem kognitiv-behavioralen Prototyp $(\mathrm{sd}=.143)$. Ein z-Test für unabhängige Stichproben zeigt hier keine signifikante Differenz $(z=-1,087, p=.86)$. Dies bedeutet, dass sich bei den psychodynamisch arbeitenden Therapeuten die Orientierung am kognitiv-behavioralen Prototyp nicht signifikant von der am psychodynamischen Ideal unterscheidet, obwohl die Korrelation mit dem psychodynamischen Prototyp deutlich geringer ist als die mit dem kognitiv-behavioralen Prototyp.

Für die kognitiv-behavioral behandelte Therapiegruppe (vgl. Tab. 13) liegt die durchschnittliche Korrelation mit dem kognitiv-behavioralen Prototyp $(\mathrm{sd}=.086)$ bei $\mathrm{r}=.632$ $(\mathrm{sd}=.05, \mathrm{z}$-Score von .749) und die durchschnittliche Korrelation mit dem psychodynamischen Prototyp $(\mathrm{sd}=.049)$ bei $\mathrm{r}=-.113(\mathrm{sd}=.05, \mathrm{z}=-.114)$. Ein $\mathrm{z}$-Test für unabhängige Stichproben zeigt hier eine signifikante Differenz an $(z=2,699, p=.004)$, was bedeutet, dass sich die kognitiv-behavioral arbeitenden Therapeuten signifikant mehr am kognitiv-behavioralen als am psychodynamischen Prototyp orientieren.

Weiterhin zeigt sich, dass sich weder die durchschnittliche Korrelation mit dem kognitivbehavioralen Prototyp noch die mit dem psychodynamischen Prototyp zwischen den beiden Therapiearmen unterscheidet (für die Korrelation mit dem psychodynamischen Prototyp: $\mathrm{z}=$ $0,349, \mathrm{p}=.363$ und für die Korrelation mit dem kognitiv-behavioralen Prototyp $\mathrm{z}=-0,793, \mathrm{p}$ $=.785)$.

Tabelle 13: Korrelationen (Pearson) mit den Prototypen und ihre z-Standardisierung.

\begin{tabular}{|l|l|l|}
\hline Stichprobe & $\begin{array}{l}\text { Psychodynamischer } \\
\text { Prototyp }\end{array}$ & $\begin{array}{l}\text { Kognitiv-behavioraler } \\
\text { Prototyp }\end{array}$ \\
\hline $\begin{array}{l}\text { Psychodynamische } \\
\text { Behandlung }\end{array}$ & $\mathbf{r}=.004$ & $\mathbf{r}=.442$ \\
$\mathrm{sd}=.11$ & $\mathrm{sd}=.11$ \\
\hline Kognitiv-behaviorale & $\mathbf{r}=\mathbf{- 1 1 3}$ & $\mathbf{r}=.632$ \\
Behandlung & $\mathrm{sd}=.05$ & $\mathrm{sd}=.05$ \\
\hline
\end{tabular}

Insgesamt ähneln demnach beide Therapieansätze stärker dem kognitiv-behavioralen als dem psychodynamischen Prototyp. Ein signifikanter Unterschied ließ sich nur für die kognitiv-behaviorale Therapiegruppe finden. Die Hypothesen, dass die implementierte psychodynamische Therapie stärker dem psychodynamischen Prototyp und die implementierte kognitiv-behaviorale Psychotherapie stärker dem kognitiv-behavioralen Prototyp ähnelt, konnte damit also nicht bestätigt werden. 


\section{Zusammenhang zwischen der Stärke der Orientierung am zugehörigen}

\section{Prototyp und dem Therapieoutcome: Überprüfung der Fragestellung A}

Zur Beantwortung dieser Fragestellung werden die Prä-Post-Differenzen der entsprechenden Outcome-Maße (zu den Daten vgl. Winkelbach, 2007) mit den vorher bestimmten z-Werten (vgl. Tab. 12) korreliert. Die Korrelation dieser z-Werte mit den einzelnen Outcome-Maßen gibt Aufschluss darüber, ob die Orientierung an einem der beiden Prototypen mit einer signifikanten Verbesserung beim Patienten einhergeht.

Tabelle 14: Korrelationen zwischen der Orientierung am jeweiligen Prototyp mit den Outcome-Maßen.

\begin{tabular}{|c|c|c|}
\hline \multirow[t]{2}{*}{ Outcome-Maß } & \multicolumn{2}{|c|}{$\begin{array}{l}\text { Pearson-Korrelation der z-Werte mit den Prä-Post- } \\
\text { Differenzen der Outcome-Maße }\end{array}$} \\
\hline & $\begin{array}{l}\text { Orientierung am } \\
\text { psychodynamischen } \\
\text { Prototyp }\end{array}$ & $\begin{array}{l}\text { Orientierung am kognitiv- } \\
\text { behavioralen Prototyp }\end{array}$ \\
\hline \multicolumn{3}{|c|}{ Psychodynamische Stichprobe } \\
\hline BAI & .094 & .008 \\
\hline PSWQ & -.182 & .132 \\
\hline STAI & -.252 & .415 \\
\hline BDI & .021 & .192 \\
\hline IIP & .094 & .117 \\
\hline HАM-A & -.363 & .258 \\
\hline HAM-D & -.012 & .270 \\
\hline \multicolumn{3}{|c|}{ Kognitiv-behaviorale Stichprobe } \\
\hline BAI & -.022 & -.313 \\
\hline PSWQ & $.421 *$ & -.222 \\
\hline STAI & .257 & -.328 \\
\hline BDI & .087 & $-.586^{* *}$ \\
\hline IIP & .129 & -.168 \\
\hline НАMА & .402 & -.354 \\
\hline HAMD & .266 & -.211 \\
\hline
\end{tabular}

Anm: $* p<.05, * * p<.01$; BAI: Beck Angst Inventar, PSWQ: Penn State Worry

Questionnaire, STAI: State Trait Angst Inventar, BDI: Beck Depression Inventar, IIP:

Inventar Interpersoneller Probleme, HAMA: Hamilton Angst Skala, HAMD: Hamilton Depression Skala.

In der psychodynamisch behandelten Stichprobe ist eine Orientierung am psychodynamischen Prototyp in keinem einzigen Fall signifikant mit einem der sieben Outcome-Maße korreliert. Das gleiche gilt für die Orientierung am kognitiv-behavioralen Prototyp (vgl. Tab. 14).

Für die kognitiv-behavioral behandelte Stichprobe hingegen gibt es eine signifikante positive Korrelation zwischen der Orientierung am psychodynamischen Prototyp und dem 
Therapieoutcome im PSWQ. Außerdem zeigt sich ein negativer hoch signifikanter Zusammenhang zwischen der Orientierung am kognitiv-behavioralen Prototyp und der PräPost-Differenz im BDI. Alle anderen Korrelationen erweisen sich als nicht signifikant.

Tabelle 15 zeigt die Korrelationen der Variable „Orientierung am eigenen Prototyp“ (vgl. Abschnitt 4 in Kapitel III zur Berechnung dieser) mit den Outcome-Maßen der Therapie. Die Variable „Orientierung am eigenen Prototyp“ besteht aus den z-Werten der Korrelationen der PQS-Ratings der 19 psychodynamisch behandelten Patienten mit dem psychodynamischen Prototyp und den z-Werten der Korrelationen der PQS-Ratings der 23 kognitiv-behavioral behandelten Patienten mit dem kognitiv-behavioralen Prototyp. Anders als zuvor werden hier also die Korrelationen zwischen den Outcome-Maßen und den z-Werten für alle 42 Patienten berechnet. Dabei zeigt sich, dass die Orientierung am eigenen Prototyp nur mit dem Outcome im PSWQ signifikant positiv korreliert ist, sonst jedoch mit keinem weiteren Outcome-Maß. Je mehr sich die Therapeuten demnach an ihrem eigenen Prototyp orientieren, umso höher ist die Prä-Post-Differenz im PSWQ.

Tabelle 15: Korrelationen zwischen „Orientierung am eigenen Prototyp“ und den OutcomeMaßen.

\begin{tabular}{|l|l|}
\hline $\begin{array}{l}\text { Outcome- } \\
\text { Maß }\end{array}$ & $\begin{array}{l}\text { Pearson-Korrelation der z-Werte mit dem eigenen Prototyp und den Prä- } \\
\text { Post-Differenzen der Outcome-Maße }\end{array}$ \\
\hline BAI & -.086 \\
\hline PSWQ & $.312^{*}$ \\
\hline STAI & .217 \\
\hline BDI & .222 \\
\hline IIP & .122 \\
\hline HAMA & .051 \\
\hline HAMD & .234 \\
\hline
\end{tabular}

Anm: ${ }^{*} \mathrm{p}<.05$; BAI: Beck Angst Inventar, PSWQ: Penn State Worry Questionnaire, STAI: State Trait Angst Inventar, BDI: Beck Depression Inventar, IIP: Inventar Interpersoneller Probleme, HAMA: Hamilton Angst Skala, HAMD: Hamilton Depression Skala.

\section{PQS-Items als Prädiktoren des Therapieerfolgs: Überprüfung der}

\section{Fragestellung B}

Um zu untersuchen, ob bestimmte Items aus dem PQS den Therapieerfolg vorhersagen können, werden lineare Regressionen berechnet. Dazu wird aufgrund der relativ kleinen Stichprobengröße $(\mathrm{N}=42)$ zunächst für jedes einzelne der zehn charakteristischsten bzw. uncharakteristischsten Items der jeweilig interessierenden Stichprobe (gesamte Stichprobe oder getrennt nach den beiden Therapiegruppen, vgl. Anhang A) eine lineare Regression 
gerechnet. Die Abhängige Variable in diesen Regressionen ist jeweils die Prä-Post-Differenz des entsprechenden Outcome-Maßes (HAMA, HAMD, BDI, BAI, STAI, PSWQ, IIP; vgl. Abschnitt 2, Kapitel III; zu den Daten vgl. Winkelbach, 2007). Die Unabhängige Variable ist zunächst jeweils eines der charakteristischsten bzw. uncharakteristischsten Items. All diejenigen Items, die in diesen Regressionen signifikant zur Aufklärung der Varianz in der AV beitragen können, werden dann gleichzeitig in ein multiples Regressionsmodell aufgenommen. Das Signifikanzniveau wird auf $\alpha=.01$ festgelegt.

Tabelle 16: Die jeweils zehn charakteristischsten und uncharakteristischsten Items der verschiedenen Teilstichproben.

\begin{tabular}{|l|l|l|}
\hline $\begin{array}{l}\text { Gesamte Stichprobe } \\
\text { (N =42) }\end{array}$ & $\begin{array}{l}\text { Psychodynamische Subgruppe } \\
\text { (n= 19) }\end{array}$ & $\begin{array}{l}\text { Kognitiv-behaviorale Subgruppe } \\
\text { (n= 23) }\end{array}$ \\
\hline Charakteristischste Items & \multicolumn{2}{|l|}{} \\
\hline Q 3 & Q 65 & Q 3 \\
\hline Q 69 & Q 3 & Q 69 \\
\hline Q 65 & Q 69 & Q 23 \\
\hline Q 23 & Q 23 & Q 88 \\
\hline Q 88 & Q 80 & Q 65 \\
\hline Q 80 & Q 88 & Q 30 \\
\hline Q 46 & Q 35 & Q 46 \\
\hline Q 30 & Q 6 & Q 80 \\
\hline Q 35 & Q 46 & Q 45 \\
\hline Q 54 & Q 54 & Q 72 \\
\hline Uncharakteristischste Items & \\
\hline Q 67 & Q 33 & Q 42 \\
\hline Q 42 & Q 38 & Q 29 \\
\hline Q 33 & Q 42 & Q 67 \\
\hline Q 29 & Q 29 & Q 33 \\
\hline Q 36 & Q 36 & Q 36 \\
\hline Q 49 & Q 49 & Q 90 \\
\hline Q 90 & Q 90 & Q 49 \\
\hline Q 91 & Q 91 & Q 2 \\
\hline Q 2 & Q 11 & Q 91 \\
\hline Q 11 & Q 2 & Q 11 \\
\hline
\end{tabular}

Ergebnisse der Regression für die Outcomevariable Hamilton Angst Skala (HAMA)

Innerhalb der gesamten Stichprobe $(\mathrm{N}=42)$ kann kein einziges der jeweils zehn charakteristischsten und uncharakteristischsten Items das Therapieoutcome gemäß Hamilton Angst Skala signifikant vorhersagen ( $\mathrm{R}^{2}$ von .000 bis .060; vgl. Anhang B, Tab. 8) (vgl. Tab. 17). 
Das gleiche gilt für die jeweils zehn charakteristischsten und uncharakteristischsten Items der kognitiv-behavioral behandelten $\left(\mathrm{R}^{2}\right.$ von .000 bis .148; vgl. Anhang B, Tab. 9) und der psychodynamisch behandelten Subgruppe ( $\mathrm{R}^{2}$ von .000 bis .257; vgl. Anhang B, Tab. 10) (vgl. Tab. 17).

Ergebnisse der Regression für die Outcomevariable Hamilton Depression Skala (HAMD)

Die Ergebnisse dieser linearen Regressionen zeigen, dass weder in der gesamten $\left(\mathrm{R}^{2}\right.$ von .000 bis .089; vgl. Anhang B, Tab. 11) noch in der psychodynamisch $\left(\mathrm{R}^{2}=.000\right.$ bis .192; vgl. Anhang B, Tab. 12) oder kognitiv-behavioral behandelten Patientengruppe $\left(\mathrm{R}^{2}=.000\right.$ bis .162; vgl. Anhang B, Tab. 13) auch nur eines der jeweils zehn charakteristischsten bzw. uncharakteristischsten Items einen signifikanten Beitrag zur Aufklärung der Varianz in der AV „Prä-Post-Differenz in der Hamilton Depression Skala“ leisten kann (vgl. Tab. 17).

Tabelle 17: Ergebnisse der linearen Regressionen für alle Outcome-Maße und alle untersuchten Stichproben.

\begin{tabular}{|c|c|c|c|}
\hline \multirow[t]{2}{*}{ Outcome-Maß } & \multicolumn{3}{|c|}{ Stichprobe } \\
\hline & $\mathrm{N}=42$ & Kognitiv-behaviorale Therapie & Psychodynamische Therapie \\
\hline \multicolumn{4}{|l|}{ HAMA } \\
\hline \multicolumn{4}{|l|}{ HAMD } \\
\hline BAI & & Q 2: $\mathrm{R}^{2}=.282, \beta=.531^{*}$ & \\
\hline \multicolumn{4}{|l|}{ BDI } \\
\hline \multicolumn{4}{|l|}{ STAI } \\
\hline PSWQ & & & Q 90 : $\mathrm{R}^{2}=.406, \beta=-.637^{*}$ \\
\hline IIP & & & \\
\hline
\end{tabular}

Ergebnisse der Regression für die Outcomevariable Beck Angst Inventar (BAI)

In der gesamten Stichprobe $(\mathrm{N}=42)$ erweist sich keines der Items als signifikanter Prädiktor des Therapieerfolgs im Beck Angst Inventar ( $\mathrm{R}^{2}$ von .000 bis .123; vgl. Anhang B, Tab. 14) (vgl. Tab. 17).

Dies gilt auch für die psychodynamisch behandelte Patientengruppe, wo es ebenfalls keinen signifikanten Prädiktor des Therapieerfolgs im BAI gibt ( $\mathrm{R}^{2}$ von .000 bis .268; vgl. Anhang B, Tab. 15) (vgl. Tab. 17).

In der kognitiv-behavioral behandelten Subgruppe hingegen erweist sich Item Q 2 (,Der Therapeut lenkt die Aufmerksamkeit auf das nonverbale Verhalten des Patienten, zum Beispiel auf die Körperhaltung oder Gesten. “) als signifikanter Prädiktor $(p=.009)$ des 
Therapieerfolgs im BAI ( $\mathrm{R}^{2}$ von .000 bis .282; vgl. Anhang B, Tab. 16; vgl. Tab. 17). Das Modell mit Item Q 2 als Prädiktor $(\mathrm{df}=22, \mathrm{p}=.009, \beta=.531)$ kann $28 \%\left(\mathrm{R}^{2}=.282\right)$ der Varianz in der AV aufklären (vgl. Tab. 17). Je mehr der Therapeut die Aufmerksamkeit auf das nonverbale Verhalten des Patienten lenkt, umso höher ist die Prä-Post-Differenz im BAI.

Ergebnisse der Regression für die Outcomevariable Beck Depression Inventar (BDI)

Die Ergebnisse dieser linearen Regressionen zeigen, dass sowohl in der gesamten Stichprobe ( $\mathrm{R}^{2}$ von .000 bis .088; vgl. Anhang B, Tab. 17) als auch in der psychodynamisch ( $\mathrm{R}^{2}$ von .001 bis .132; vgl. Anhang B, Tab. 18) und in der kognitiv-behavioral behandelten Subgruppe ( $\mathrm{R}^{2}$ von .001 bis .268; vgl. Anhang B, Tab. 19) keines der jeweils zehn charakteristischsten und uncharakteristischsten Items einen signifikanten Beitrag zur Aufklärung der Varianz in der AV „Prä-Post-Differenz im Beck Depression Inventar“ leisten kann (vgl. Tab. 17).

\section{Ergebnisse der Regression für die Outcomevariable State Trait Angst Inventar (STAI)}

Sowohl in der gesamten Stichprobe ( $\mathrm{R}^{2}$ von .000 bis .106; vgl. Anhang B, Tab. 20) als auch in der psychodynamisch $\left(\mathrm{R}^{2} .001\right.$ bis .182; vgl. Anhang B, Tab, 21) sowie ebenfalls in der kognitiv-behavioral behandelten ( $\mathrm{R}^{2}$ von .000 bis .159; vgl. Anhang B, Tab. 22) Subgruppe zeigt sich jeweils keines der zwanzig Items als signifikanter Prädiktor der AV „Prä-Post-Differenz im STAI“ (vgl. Tab. 17).

Ergebnisse der Regression für die Outcomevariable Penn State Worry Questionnaire (PSWQ)

Sowohl in der gesamten Stichprobe ( $\mathrm{R}^{2}$ von .000 bis .129, vgl. Anhang B, Tab. 23) als auch in der kognitiv-behavioral behandelten Subgruppe ( $\mathrm{R}^{2}$ von .000 bis .159) trägt keines der Items signifikant zur Aufklärung der Varianz in der AV bei (vgl. Anhang B, Tab. 24).

In der psychodynamisch behandelten Subgruppe ( $\mathrm{R}^{2}$ von .000 bis .406 , vgl. Anhang B, Tab. 25) hingegen erweist sich Item Q 90 (,,Träume und Phantasien des Patienten werden besprochen.“) als signifikant ( $\mathrm{p}=.003)$. Das Modell mit Item Q 90 als Prädiktor $(\mathrm{df}=18, \mathrm{p}=$ $.003, \beta=-.637)$ kann $41 \%\left(\mathrm{R}^{2}=.406\right)$ der Varianz in der AV aufklären (vgl. Tab. 17). Je weniger über Träume und Phantasien gesprochen wird, umso höher ist der Therapieerfolg gemessen mit dem PSWQ.

Ergebnisse der Regression für die Outcomevariable Inventar Interpersonaler Probleme (IIP)

Für die gesamte Stichprobe $(\mathrm{N}=42)$ zeigt sich keines der charakteristischsten bzw. uncharakteristischsten Items als signifikanter Prädiktor des Therapieerfolgs im IIP ( $\mathrm{R}^{2}$ von 
.000 bis .089; vgl. Anhang B, Tab. 26). Das gleiche gilt für die kognitiv-behavioral ( $\mathrm{R}^{2}$ von .000 bis .134; vgl. Anhang B, Tab. 27) und auch für die psychodynamisch behandelte Subgruppe ( $\mathrm{R}^{2}$ von .001 bis .224, vgl. Anhang B, Tab. 28).

Ergebnisse der Multiplen Regressionen zu den zehn charakteristischsten Items

Da sich in den vorangegangen Analysen nur zwei Items als signifikante Prädiktoren des Therapieerfolgs ausgewiesen haben, wird nun noch zusätzlich überprüft, ob die jeweils zehn charakteristischsten Items insgesamt den Therapieerfolg signifikant vorhersagen können. Dazu werden getrennt nach den beiden Teilstichproben (psychodynamisch vs. kognitivbehavioral behandelt) und für jedes der sieben Outcome-Maße separat multiple Regressionen berechnet. UVs sind die jeweils zehn charakteristischen Items der beiden Subgruppen, die simultan in das Modell aufgenommen werden, AV ist die Prä-Post-Differenz des jeweiligen Outcome-Maßes (Tab. 18). Kein einziges Modell, in das die zehn charakteristischsten Items eingehen, ist signifikant, ungeachtet der Art der Behandlung und des betrachteten OutcomeMaßes.

Tabelle 18: Ergebnisse der multiplen Regressionen zu den jeweils zehn charakteristischsten Items.

\begin{tabular}{|l|l|l|}
\hline $\begin{array}{l}\text { AV: Prä-Post-Differenz des } \\
\text { Outcome-Maßes }\end{array}$ & $\begin{array}{l}\text { UVs: 10 für die } \\
\text { psychodynamische } \\
\text { Behandlung } \\
\text { charakteristischste Items }\end{array}$ & $\begin{array}{l}\text { UVs: 10 für die kognitiv- } \\
\text { behaviorale Behandlung } \\
\text { charakteristischste Items }\end{array}$ \\
\hline HAMA & $\mathrm{p}=.41$ & $\mathrm{p}=.84$ \\
& $\mathrm{R}^{2}=.598$ & $\mathrm{R}^{2}=.321$ \\
\hline HAMD & $\mathrm{p}=.51$ & $\mathrm{p}=.82$ \\
& $\mathrm{R}^{2}=.556$ & $\mathrm{R}^{2}=.336$ \\
\hline STAI & $\mathrm{p}=.64$ & $\mathrm{p}=.82$ \\
& $\mathrm{R}^{2}=.498$ & $\mathrm{R}^{2}=.316$ \\
\hline PSWQ & $\mathrm{p}=.87$ & $\mathrm{p}=.38$ \\
& $\mathrm{R}^{2}=.368$ & $\mathrm{R}^{2}=.498$ \\
\hline BAI & $\mathrm{p}=.64$ & $\mathrm{p}=.08$ \\
& $\mathrm{R}^{2}=.500$ & $\mathrm{R}^{2}=.667$ \\
\hline BDI & $\mathrm{p}=.82$ & $\mathrm{p}=.47$ \\
& $\mathrm{R}^{2}=.405$ & $\mathrm{R}^{2}=.463$ \\
\hline IIP & $\mathrm{p}=.42$ & $\mathrm{p}=.94$ \\
& $\mathrm{R}^{2}=.594$ & $\mathrm{R}^{2}=.236$ \\
\hline
\end{tabular}

UV: Unabhängige Variable, BAI: Beck Angst Inventar, PSWQ: Penn State Worry Questionnaire, STAI: State Trait Angst Inventar, BDI: Beck Depression Inventar, IIP: Inventar Interpersoneller Probleme, HAMA: Hamilton Angst Skala, HAMD: Hamilton Depression Skala. 


\section{Diskussion}

Die vorliegende Arbeit beschäftigt sich mit der Frage, inwieweit sich die Therapieprozesse einer kognitiv-behavioralen und einer psychodynamischen Psychotherapie bei Patienten mit einer Generalisierten Angststörung bezüglich zentraler therapeutischer Prozessvariablen unterscheiden. Auch wurde überprüft, inwiefern sich diese beiden Therapieprozesse an einer prototypischen Therapiesitzung einer kognitiv-behavioralen bzw. einer psychodynamischen Therapie orientieren und ob diese Orientierung mit einem positiven Ergebnis der Therapie in Zusammenhang steht. Weiterhin wurde untersucht, ob bestimmte Prozessvariablen den Therapieerfolg vorhersagen können.

Hierzu wurden Patienten mit der Diagnose einer Generalisierten Angststörung in einem naturalistischen Setting randomisiert entweder einer kognitiv-behavioralen oder einer psychodynamischen Psychotherapie zugeteilt. Die beiden implementierten Therapien wurden jeweils in einem Manual spezifiziert (vgl. Leibing et al., 2003; Leichsenring et al., 2005). Der Therapieprozess der auf Tonband aufgenommenen Therapiesitzungen wurde dann anhand des Psychotherapie Prozess Q-Sort aus der Perspektive nichtteilnehmender Beobachter eingeschätzt. Dabei wurde jeweils immer eine komplette Sitzung beurteilt, wodurch sich einzelne Ereignisse im Therapieprozess besser in den Kontext einordnen lassen. Durch die verwendeten Manuale wurde dabei eine Replikation der Studie ermöglicht. Anders als in vielen älteren Studien wurden ausschließlich Patienten mit einer Generalisierten Angststörung behandelt. Dadurch können aus den vorliegenden Befunden spezifische Erkenntnisse für diese Patientengruppe gewonnen werden. Die Therapien wurden von insgesamt neun verschiedenen Therapeuten durchgeführt, was die Repräsentativität der Befunde ebenfalls erhöht.

\section{Zur Reliabilität der Prozessbeurteilungen der Rater}

Die Reliabilität für die Prozessbeurteilungen ist sehr zufrieden stellend (.84 - .86), was durch ein umfangreiches Training der Rater sowie durch einen Prozess der Konsensfindung erreicht werden konnte. Die erzielten Übereinstimmungswerte stimmen damit mit denen überein, die der Entwickler des PQS selbst angegeben hatte (zwischen .83 und .89 für zwei und zwischen .89 und .92 für drei Beurteiler; vgl. Jones et al., 1988). Somit liegt die Beobachterübereinstimmung in der vorliegenden Studie deutlich über den Mindestanforderungen für Beobachtermaße in der Therapieprozessforschung von .70 (vgl. Orlinsky \& Howard, 1987). 
Ein statistischer Vergleich der hier erzielten Beobachterübereinstimmungen in den Prozessbeurteilungen für die psychodynamisch und die kognitiv-behavioral behandelte Subgruppe zeigt keinen bedeutsamen Unterschied. Demnach ist es den Ratern in beiden Therapieansätzen gleichermaßen gut gelungen, die unterschiedlichen Therapieprozesse zu erfassen.

\section{Zum multiplen Testen in der vorliegenden Untersuchung}

Aufgrund des multiplen Testens (also z.B. wird für jedes der 100 Prozessmerkmale aus dem PQS ein einzelner t-Test zum Vergleich zwischen 10. und 18. Sitzung durchgeführt) besteht in der vorliegenden Arbeit die Gefahr der $\alpha$-Fehler-Kumulierung. Dies bedeutet, dass das Risiko eines $\alpha$-Fehlers, d.h. Unterschiede in der Stichprobe zu finden, die in der Population nicht vorhanden sind, mit jedem der multiplen Tests steigt. Solche $\alpha$-FehlerKumulierungen treten generell auf, wenn eine ,globale“ Hypothese anhand mehrerer Signifikanztests überprüft wird. In der Fachliteratur (z.B. Bortz \& Döring, 1993) wird dann die so genannte Bonferroni-Adjustierung vorgeschlagen. Hier wird das Signifikanzniveau für die einzelnen Tests als Quotient aus einem gängigen $\alpha$-Signifikanzniveau und der Anzahl der durchzuführenden Tests bestimmt. Soll eine ,globale“ Nullhypothese über verschiedene Einzeltests auf einem vorher festgelegten $\alpha$-Niveau verworfen werden, muss mindestens einer dieser Einzeltests die so berechnete „korrigierte“ Irrtumswahrscheinlichkeit erreichen.

In der vorliegenden Untersuchung wurde sich gegen die Verwendung der BonferroniAdjustierung für multiple Vergleiche entschieden. Nachteil dieser Korrektur ist, dass dadurch die Power bzw. Teststärke der Tests sinkt, die hier auch aufgrund der kleinen Stichprobe schon eher gering ist. Die Teststärke gibt die Wahrscheinlichkeit an, mit der eine richtige Alternativhypothese durch einen Signifikanztest entdeckt wird. Durch die $\alpha$-Adjustierung sinkt also die Wahrscheinlichkeit, tatsächlich vorhandene Effekte durch die statistischen Tests aufdecken zu können.

Stattdessen wurde das Signifikanzniveau für die multiplen Tests hier auf $\alpha=.01$ festgelegt. Der Autor des Psychotherapie Prozess Q-Sort selbst (vgl. Jones et al., 1993) hatte dieses Vorgehen vorgeschlagen. Damit steigt zwar das Risiko eines $\alpha-$ Fehlers. Dies ist aber deshalb weniger problematisch, weil Effekte, die aufgrund eines $\alpha$-Fehlers zunächst in der Stichprobe entdeckt wurden, in weiteren Untersuchungen weiter überprüft und dann ggf. wieder verworfen werden können. ß-Fehler hingegen, d.h. Effekte, die zwar in der Population vorhanden sind, aber in der Stichprobe nicht gefunden werden konnten, würden nicht entdeckt 
werden, da keine weiteren Untersuchungen stattfänden. Solche $\beta$-Fehler werden mit der $\alpha$ Adjustierung wahrscheinlicher.

In der vorliegenden Untersuchung konnten einige Effekte in der Stichprobe gefunden werden. Für die nun folgende Diskussion dieser Ergebnisse ist aus den genannten Gründen deshalb zu beachten, dass sie aufgrund der $\alpha$ - Kumulierung zunächst als vorläufige Befunde betrachtet und in weiteren Untersuchungen nochmals überprüft werden sollten.

\section{Der Therapieprozess in der 10. und 18. Sitzung: Gibt es Unterschiede?}

Ablon und Jones (1999) verglichen den Therapieprozess mittels einer frühen (4.) und einer späten (12. von insgesamt 16) Sitzung und fanden keine bedeutsamen Unterschiede. In der vorliegenden Studie wurden die Therapieprozesse zweier Sitzungen aus der mittleren Phase der Therapie (Sitzung 10 und 18) einander gegenübergestellt. In der Anfangsphase einer Therapie stehen v.a. Beziehungsgestaltung sowie Problem- und Bedingungsanalyse und in der Endphase Rückfallprävention und Verfestigung der erreichten Ergebnisse im Vordergrund. In der mittleren Phase einer Therapie hingegen wird stärker die zentrale Problematik des Patienten fokussiert. Die Behandlung dieser zentralen Problematik ist hier in den verwendeten Manualen (Leibing et al., 2003; Leichsenring et al., 2005) sowohl für Sitzung 10 als auch für Sitzung 18 vorgesehen. Aus diesem Grund wurden auch keine wesentlichen Unterschiede hinsichtlich der drei zentralen Bereiche des Therapieprozesses (emotionaler Zustand bzw. Haltung des Patienten in der Therapie, Interaktion zwischen Patient und Therapeut und therapeutische Interventionen) zwischen den beiden Sitzungen erwartet.

Erwartungsgemäß finden sich in der vorliegenden Studie dann auch keine bedeutsamen Unterschiede zwischen den Therapieprozessen von der 10. und 18. Sitzung. Dies zeigt sich einerseits darin, dass sich beide Zeitpunkte nahezu mit den gleichen charakteristischen und uncharakteristischen Prozessmerkmalen beschreiben lassen und andererseits darin, dass es bei keinem einzigen dieser Prozessmerkmale bedeutsame Mittelwertsunterschiede zwischen den beiden Messzeitpunkten gab. Dies war ebenso dann der Fall, wenn die beiden Therapiearme jeweils separat untersucht wurden. Dies bedeutet, dass sich die Therapieprozesse zu den beiden Zeitpunkten weder in Bezug auf den emotionalen Zustand des Patienten bzw. seine Haltung in der Therapie noch inhaltlich bzw. in Bezug auf die Interaktion von Patient und Therapeut noch im Hinblick auf therapeutische Interventionen bedeutsam voneinander unterscheiden. 
Die hier untersuchte mittlere Phase des Therapieprozesses zeichnet sich dadurch aus, dass Therapeut und Patient konzentriert am spezifischen Problem des Patienten arbeiten (Q 23, Q 88). Dabei beziehen sie sich auf die aktuelle oder kurz zurückliegende Lebenssituation des Patienten (Q 69). Der Therapeut unterstützt den Patienten in dieser Fokussierung (Q 65, Q 80, Q 3).

Die vorliegenden Befunde erlauben damit die Vorgehensweise, nach der in die weiteren statistischen Analysen jeweils der Mittelwert für jeden Patienten aus den beiden Sitzungen 10 und 18 einging.

\section{Der Therapieprozess in der psychodynamischen und kognitiv- behavioralen Therapie: Gibt es Unterschiede?}

Die durch das Manual operationalisierte kognitiv-behaviorale Therapie der GAS (Leibing et al., 2003) zielt in erster Linie auf eine Reduktion der vegetativen Erregung, einen Abbau der kognitiven Verzerrungen sowie insgesamt auf eine Verbesserung der Problem- und Stressbewältigung. Auch soll ein Abnahme des ,,worrying“ und des Vermeidungs- und Rückversicherungsverhaltens sowie eine Veränderung bestimmter negativer kognitiver Grundannahmen erreicht werden (vgl. Leibing et al., 2003). Der Therapeut soll in der Therapie eine aktive, manchmal auch direktive Haltung dem Patienten gegenüber einnehmen, Informationen und Hinweise auf günstigere Verhaltensweisen geben und spezifische Aktivitäten anregen. Auch soll er sich eindeutig, supportiv und für den Klienten transparent verhalten.

Mit Hilfe der durch das Manual operationalisierten psychodynamischen Therapie (Leichsenring et al., 2005) sollen v.a. typische primitive Wünsche, Widerstände und Abwehrhaltungen sowie vergangene traumatische Ereignisse bearbeitet werden. Dies soll im Rahmen einer sicheren und hilfreichen Therapiebeziehung als korrigierende emotionale Erfahrung geschehen (vgl. Crits-Christoph et al., 1995).

Wegen der Unterschiede, die die beiden hier gewählten Therapieansätze sowohl im Hinblick auf ihre theoretische Konzeption als auch auf die hier spezifische Zielstellung zur Behandlung der GAS zeigen, wurden bedeutsame Unterschiede zwischen den Therapieprozessen dieser beiden Therapien erwartet. Diese sollten sich v.a. im Hinblick auf therapeutische Interventionen zeigen. In Bezug auf den emotionalen Zustand des Patienten bzw. sein Verhalten in der Therapie sowie auch in Bezug auf die Interaktion zwischen Patient 
und Therapeut bzw. auf die in der Sitzung thematisierten Inhalte wurden keine Unterschiede erwartet.

Zunächst werden für beide Therapieprogramme diejenigen Prozessmerkmale bestimmt, die die beiden Therapien jeweils am besten beschreiben können. Dazu wird eine Rangreihe mit den zehn charakteristischsten und den zehn uncharakteristischsten Prozessmerkmalen gebildet. Dabei zeigt sich, dass bei beiden Therapien sieben von zehn der charakteristischen Prozessmerkmale identisch sind, was bedeutet, dass sich sowohl der psychodynamische als auch der kognitiv-behaviorale Therapieprozess mit fast identischen Therapieprozessmerkmalen beschreiben lässt. In beiden Therapien bringt der Patient relevantes Material zur Sprache (Q 88). Es wird v.a. die aktuelle oder kurz zurückliegende Lebenssituation des Patienten besprochen (69) und spezifische Themen werden fokussiert (Q 23). Die Therapeuten beider Therapieansätze versuchen dem Patienten, das Erzählen zu erleichtern (Q 3), klarifizieren oder formulieren neu (Q 65), bieten Erfahrungen oder Ereignisse aus einer anderen Perspektive an (Q 80) und sprechen klar und verständlich (Q 46). Zu den zehn charakteristischsten Prozessmerkmalen der hier operationalisierten psychodynamischen Therapie zählt weiterhin die klare und strukturierte Ausdrucksweise des Patienten (Q 54), die Fokussierung des Selbstbilds des Patienten (Q 35) und die Empathie des Therapeuten (Q 6). Die hohe Empathie des Therapeuten sowie sein Bestreben, dem Patienten das Erzählen zu erleichtern, können als Versuch, eine sichere und hilfreiche Therapiebeziehung zu etablieren, verstanden werden. Dadurch, dass der Therapeut klar und verständlich spricht, v.a. im Hier und Jetzt bleibt und spezifische Themen wie z.B. das Selbstbild des Patienten besprochen werden, ist es dem Patienten möglich, auch schwierige Themen klar und deutlich anzusprechen. Die Klarifikationen bzw. Wiederholungen des Therapeuten sowie die veränderte Perspektive, aus der er Erfahrungen oder Ereignisse anbietet, dienen dazu, dem Patienten auch Unbewusstes bewusst zu machen.

Die hier kognitiv-behavioral behandelten Patienten können das Wesen ihrer Therapie gut verstehen (Q 72). In der Therapie werden v.a. kognitive Themen besprochen (Q 30). Der Therapeut verhält sich supportiv (Q 45). Die Fokussierung spezifischer kognitiver Themen stimmt mit der Zielstellung der Therapie überein, nach der kognitive Verzerrungen bzw. ungünstige Grundannahmen verändert werden sollen. Auch sollte der Therapeut sich dabei supportiv verhalten, was er getan hat (z.B. auch durch Äußerungen, die dem Patienten das Erzählen erleichtern sollen). Auch die Forderung nach Transparenz im Vorgehen und 
Verhalten des Therapeuten ist erfüllt, was sich darin zeigt, dass der Patient das Wesen seiner Therapie sehr gut verstehen kann. Auch spricht er relevante Themen (z.B. ,worrying“) an.

In der Rangreihung der zehn uncharakteristischen Prozessaspekte treten bei beiden Therapieansätzen neun identische Merkmale auf, d.h. auch in den Aspekten, die für die jeweiligen Therapien am untypischsten sind, unterscheiden sie sich nicht voneinander. Die Patienten sprechen nicht von Distanz oder Nähe (Q 29, Q 33), weisen die Bemerkungen des Therapeuten nicht zurück (Q 42) und erleben dem Therapeuten gegenüber keine ambivalenten Gefühle (Q 49). Insgesamt wird in den Sitzungen weder über sexuelle Gefühle und Erfahrungen (Q 11) noch über Träume und Phantasien (Q 90) oder die Kindheit (Q 91) gesprochen. Die Therapeuten lenken die Aufmerksamkeit nur wenig auf das nonverbale Verhalten des Patienten (Q 2) und weisen auch nur wenig auf Abwehrmechanismen hin (Q36). Wie erwartet interpretiert der kognitiv-behavioral orientierte Therapeut kein unbewusstes Material (Q 67). Ebenfalls wie erwartet werden in der psychodynamischen Therapie keine Aktivitäten, die der Patient außerhalb der Therapie in Angriff nehmen soll, besprochen (Q 38).

Neben der Betrachtung der charakteristischen und uncharakteristischen Prozessmerkmale werden statistische Vergleiche für jedes einzelne Prozessmerkmal aus dem PQS durchgeführt, um Unterschiede zwischen den beiden Therapieansätzen identifizieren zu können. Bei 30 von 100 Prozessaspekten zeigen sich bedeutsame Unterschiede zwischen den beiden Therapiearmen (vgl. Tab. 5, Kapitel 4), was gleichzeitig auch bedeutet, dass es bei 70 von 100 Merkmalen keine bedeutsamen Differenzen zwischen der psychodynamischen und der kognitiv-behavioralen Therapie gab.

Von diesen 70 Therapieprozessaspekten, die nicht zwischen den beiden Therapiearmen unterscheiden konnten, beschreiben 33 die Haltung, den emotionalen Zustand oder das Erleben des Patienten in der therapeutischen Beziehung (Q 1, Q 5, Q 7, Q 10, Q 13, Q 14, Q 15, Q 20, Q 25, Q 26, Q 29, Q 32, Q 33, Q 34, Q 42, Q 52, Q 53, Q 54, Q 55, Q 56, Q 59, Q 60, Q 61, Q 70, Q 71, Q 72, Q 73, Q 78, Q 84, Q 88, Q 94, Q 95 und Q 97). Fünfzehn dieser Prozessmerkmale, in denen sich die beiden unterschiedlichen Therapieprozesse nicht unterscheiden, beschreiben den emotionalen Zustand des Patienten (Q 7, Q 13, Q 26, Q 29, Q 33, Q 34, Q 56, Q 59, Q 60, Q 61, Q 70, Q 71, Q 84, Q 94 und Q 97). Dieser Befund ist damit zu erklären, dass es sich in der vorliegenden Stichprobe ausschließlich um Patienten mit dem gleichen Störungsbild und daher ähnlichem emotionalen Befinden handelt. Die noch verbleibenden 18 Prozessmerkmale des Patienten, die sich nicht zwischen den beiden 
Therapieprozessen unterscheiden, beschreiben die Art und Weise, wie der Patient sich in der Therapie verhält bzw. wie er zum Therapeuten in Kontakt tritt.

Insgesamt gibt es im PQS 39 Prozessmerkmale, die das Verhalten oder den emotionalen Zustand des Patienten in der Therapie beschreiben. Im Hinblick auf all diejenigen im PQS berücksichtigten Therapieprozessmerkmale, die das Verhalten oder den emotionalen Zustand des Patienten fokussieren, gibt es also nur bei sechs Merkmalen signifikante Unterschiede zwischen den beiden Therapieansätzen. Die Häufigkeit von signifikanten und nichtsignifikanten Unterschieden zwischen den beiden Therapien in den Prozessmerkmalen, die den emotionalen Zustand des Patienten beschreiben, unterscheidet sich signifikant voneinander. Dies bedeutet, dass es hier deutlich mehr Ähnlichkeiten als Unähnlichkeiten im Hinblick auf diesen Bereich des Therapieprozesses gibt. Die Vermutung, dass sich die beiden Therapieansätze nicht bedeutsam in den Prozessmerkmalen, die den emotionalen Zustand des Patienten bzw. sein Verhalten in der Therapie beschreiben, unterscheiden, hat sich demnach bestätigt. Dieser Befund stimmt mit Ergebnissen anderer Studien überein, nach denen die Ähnlichkeit zwischen verschiedenen Therapieansätzen v.a. durch ähnliche Verhaltens- und Erlebensweisen des Patienten und durch dessen jeweils vergleichbare Mitarbeit in der Therapie zu begründen ist (vgl. Jones \& Pulos, 1993; Ablon \& Jones, 1999).

Die wenigen bedeutsamen Unterschiede zwischen den beiden hier gewählten Therapieansätzen in Bezug auf Prozessmerkmale, die den emotionalen Zustand des Patienten oder sein Verhalten in der Therapie beschreiben, zeigen sich v.a. dort, wo sie auch gemäß der Therapietheorie zu erwarten waren. Sie sind als Reaktion auf das Verhalten des Therapeuten bzw. seine Interventionen zu verstehen. So ist der Patient in der hier durchgeführten psychodynamischen Therapie stärker kontrollierend (Q 87), erlebt mehr Ambivalenz seinem Therapeuten gegenüber ( $\mathrm{Q} 49)$, ist stärker besorgt oder beunruhigt wegen seiner Abhängigkeit vom Therapeuten (Q 8) und weigert sich mehr, sich mit seinen eigenen Gedanken, Reaktionen oder Motiven, die mit seinen Problemen im Zusammenhang stehen, auseinanderzusetzen (Q 58). Diese Therapieprozessmerkmale können als eine Form des „Widerstands“ des Patienten gegen die Bewusstwerdung unbewusster Konflikte interpretiert werden (vgl. 1.2.1 in Kapitel II). Auch ist der psychodynamisch behandelte Patient möglicherweise gerade deshalb wachsamer und misstrauischer (Q 44). Weiterhin fordert er mehr (Q 83), was der größeren Zurückhaltung des Therapeuten in der psychodynamischen Therapie geschuldet sein könnte (vgl. Sloane et al., 1975; Stiles et al., 1988). 
Das hier verwendete kognitiv-behaviorale Therapieprogramm unterscheidet sich weiterhin ebenfalls nicht in der Mehrzahl derjenigen Prozessaspekte, die die Interaktion zwischen Patient und Therapeut oder in der Sitzung fokussierte Themen beschreiben, von dem hier verwendeten psychodynamischen Behandlungsprogramm. Von insgesamt 25 im PQS dazu berücksichtigten Merkmalen differenzieren hier 20 nicht bedeutsam zwischen den beiden Therapieansätzen (Item Q 3, Q 4, Q 11, Q 16, Q 19, Q 23, Q 24, Q 35, Q 41, Q 47, Q 63, Q 64, Q 68, Q 69, Q 74, Q 75, Q 90, Q 91, Q 92 und Q 96). Die Häufigkeit von signifikanten und nichtsignifikanten Unterschieden zwischen den beiden Therapien in den Prozessmerkmalen, die die Interaktion zwischen Patient und Therapeut beschreiben, unterscheidet sich signifikant voneinander. Dies bedeutet, dass es hier deutlich mehr Ähnlichkeiten als Unähnlichkeiten im Hinblick auf diesen Bereich des Therapieprozesses gibt. Die Vermutung, dass sich die beiden Therapieansätze nicht bedeutsam in den Prozessmerkmalen, die die Interaktion von Patient und Therapeut bzw. die in der Sitzung thematisierten Inhalte beschreiben, unterscheiden, hat sich demnach bestätigt. Ähnlich wie schon bei Ablon und Jones (1999) und Jones und Pulos (1993) zeigten sich auch in der vorliegenden Studie diesbezüglich kaum bedeutsame Unterschiede zwischen den beiden Therapien.

Auch in diesem Bereich des Therapieprozesses zeigen sich die wenigen bedeutsamen Unterschiede zwischen den beiden hier gewählten Therapieansätzen v.a. dort, wo sie auch zu erwarten waren. Insgesamt werden in dem hier verwendeten kognitiv-behavioralen Therapieprogramm theoriekonform und übereinstimmend mit den Befunden aus anderen Studien (vgl. Abschnitt 5 in Kapitel II) stärker kognitive Themen (Q 30) und auch spezifische Aktivitäten, die der Patient außerhalb der Therapie in Angriff nehmen soll (Q 38), thematisiert (vgl. Ablon \& Jones, 1999; Watzke, 2002). Ebenfalls theoriekonform wird in dem hier gewählten psychodynamischen Therapieansatz mehr über die therapeutische Beziehung gesprochen (Q 98), die hier als Agens der Veränderung gilt (vgl. 1.2.4 in Kapitel II). Auch hat die therapeutische Beziehung hier einen stärker rivalisierenden Charakter (Q 39) und es wird insgesamt mehr geschwiegen (Q 12).

Während andere Studien in Bezug auf die Exploration von Faktoren, die die Therapie evtl. stören könnten (Q 47), zeigten, dass psychodynamisch orientierte Therapeuten diese öfter explorierten als kognitiv-behavioral orientierte Therapeuten (vgl. Ablon \& Jones, 1999; Jones \& Pulos, 1993; Goldfried et al., 1998), konnten in der vorliegenden Studie für dieses Prozessmerkmal keine Unterschiede gefunden werden. Hier spielte dieses Merkmal des 
Therapieprozesses insgesamt nur eine untergeordnete Rolle mit einem durchschnittlichen PQS-Rating von 5,44, was bedeutet, dass es als eher weniger wichtig für die Sitzungen charakterisiert wurde. In beiden Therapiegruppen ist außerdem die Standardabweichung für die Beurteilung dieses Merkmals sehr gering (. 54 bzw. .45). Evtl. ist die Exploration von möglicherweise die Therapie störenden Faktoren eher zu Beginn oder am Ende einer Therapie von Bedeutung und weniger in der mittleren Phase, die hier erfasst wurde. In dieser Phase hat sich die Therapiebeziehung bereits etabliert und ist daher weniger störanfällig.

Studien von Jones und Pulos (1993) und Ablon und Jones (1998) hatten gezeigt, dass psychodynamische Therapien sich häufiger mit vergangenen Erfahrungen beschäftigten als kognitiv-behaviorale (Q 91). Dieser Befund ließ sich hier nicht replizieren, was sicherlich der Fokussierung auf das Hier und Jetzt in dem hier verwendeten psychodynamischen Therapieansatz zu schulden ist (vgl. 1.2.2 in Kapitel II). In beiden Therapiegruppen wurde dieses Merkmal als insgesamt uncharakteristisch für die Therapien eingestuft $(M=1,60, \mathrm{sd}=$ .84 für die psychodynamische und $\mathrm{M}=1,44, \mathrm{sd}=.30$ für die kognitiv-behaviorale Therapie).

Auch in Bezug auf die interpersonelle Ebene (vgl. Q 63), die nach Befunden von Jones und Pulos (1993) und Silove et al. (1990) in der psychodynamischen Therapie stärker als in der kognitiv-behavioralen Therapie fokussiert wird, fanden sich in der vorliegenden Untersuchung keine Unterschiede zwischen den beiden durchgeführten Therapien. Auch eine andere Studie hatte ebenfalls keine Unterschiede gezeigt (vgl. Kerr et al., 1992). Der Befund aus der vorliegenden Untersuchung verwundert insofern, als dass gerade in dem hier durchgeführten psychodynamischen Therapieansatz nach Leichsenring et al. (2005) interpersonelle Aspekte bei der Behandlung der GAS eine besondere Rolle spielen sollten. In beiden Therapiegruppen jedoch wird dieses Merkmal als insgesamt eher wenig wichtig für die Therapie eingeschätzt $(\mathrm{M}=6,18, \mathrm{sd}=1,38$ für die psychodynamische und $\mathrm{M}=5,61, \mathrm{sd}=$ 1,81 für die kognitiv-behaviorale Therapie).

Die meisten bedeutsamen Differenzen im Therapieprozess zwischen den hier verwendeten kognitiv-behavioralen und psychodynamischen Therapieprogrammen zeigen sich im Hinblick auf therapeutische Interventionen und die Haltung des Therapeuten. Immerhin bei 19 von insgesamt 36 Aspekten zeigen sich signifikante Unterschiede. Die Häufigkeit von signifikanten und nichtsignifikanten Unterschieden zwischen den beiden Therapien in den Prozessmerkmalen, die therapeutische Interventionen beschreiben, unterscheidet sich nicht signifikant voneinander. Dies bedeutet, dass es hier etwa gleich viele Ähnlichkeiten und Unähnlichkeiten im Hinblick auf diesen Bereich des Therapieprozesses gibt. Die Vermutung, 
dass sich die beiden Therapieansätze v.a. in den Prozessmerkmalen, die therapeutische Interventionen beschreiben, bedeutsam unterscheiden, hat sich demnach bestätigt.

Theoriekonform (vgl. Abschnitt 5 in Kapitel II; Goldfried et al., 1997, 1998; Malik et al., 2003) lenkt der psychodynamisch orientierte Therapeut in dieser Untersuchung die Aufmerksamkeit stärker auf Emotionen (Q 50, Q 81). Auch weist er häufiger auf Abwehrmechanismen des Patienten hin (Q 36) und interpretiert häufiger verdrängtes oder unbewusstes Material (Q 67). Im Vergleich dazu gibt der kognitiv-behavioral orientierte Therapeut häufiger explizit Ratschläge und Anleitungen (Q 27), erläutert eher das Rational seiner Technik (Q 57) und ermuntert den Patienten stärker zu neuen Verhaltensweisen im Umgang mit anderen (Q 85). In der vorliegenden Untersuchung sind die psychodynamisch orientierten Therapeuten weiterhin distanzierter (Q 9), neutraler (Q 93) und taktloser (Q 77) und können den therapeutischen Prozess und den emotionalen Zustand des Patienten besser einschätzen (Q 28). Die kognitiv-behavioral orientierten Therapeuten aus dieser Studie kontrollieren die Interaktion aktiver und strukturieren stärker (Q 17), verhalten sich „lehrerhafter“ (Q 37), sind supportiver (Q 45) und beruhigen den Patienten häufiger (Q 66). Ihr Kommunikationsstil ist klarer und verständlicher (Q 46) und sie sind zuversichtlicher oder selbstsicherer (Q 86). Diese Befunde stimmen mit denen vorangegangener Untersuchungen überein (vgl. Stiles et al., 1988; Goldfried et al., 1997; Malik et al., 2003).

Weiterhin sind die psychodynamisch orientierten Therapeuten in der vorliegenden Untersuchung empathischer (Q 6). In der psychodynamischen Therapie (vgl. Luborsky, 1995) wird die Herstellung einer hilfreichen Beziehung zwischen Patient und Therapeut als der wichtigste supportive Wirkfaktor verstanden (vgl. 1.2.4 in Kapitel II). Dafür ist die Empathie des Therapeuten eine unabdingbare Voraussetzung. In der kognitiv-behavioralen Therapie hingegen wird der therapeutischen Beziehung eine wichtige, wenngleich auch nicht hinreichende Wirkung bei den Veränderungen im Patienten zugesprochen. In Bezug auf die jeweiligen Therapietheorien war der Befund aus dieser Studie demnach zu erwarten. Dennoch hatte eine frühere Studie im Hinblick auf die Empathie keine Unterschiede zwischen den beiden Therapieansätzen gefunden (vgl. Wiser \& Goldfried, 1996).

In der vorliegenden Studie dienen psychodynamische Interventionen häufiger der Klarifikation, Neuformulierung oder Wiederholung von Aspekten, die der Patient bereits angesprochen hat (Q 65). Dieser Befund steht im Widerspruch zu einem Befund aus einer früheren Studie, die zwischen psychodynamischen und kognitiv-behavioralen Therapien keine Unterschiede gefunden hatte (Sloane et al., 1975). Allerdings ist es wahrscheinlich, dass 
sich die jeweils untersuchten Therapien und die zugehörigen Therapietheorien inzwischen weiterentwickelt und damit verändert haben. Auch ist nicht klar, ob in der vorliegenden Studie und in der von Sloane et al. (1975) jeweils das gleiche Prozessmerkmal erfasst wurde, da es jeweils mit anderen Instrumenten operationalisiert wurde.

Insgesamt konnten die Befunde vorangegangener Studien, die ebenfalls PQS verwendet hatten, damit in weiten Teilen repliziert werden. Die Mehrzahl an Prozessmerkmalen unterscheidet sich nicht zwischen den jeweils untersuchten Therapieansätzen. Die Befunde der vorliegenden Untersuchung stimmen mit denen von Jones und Pulos (1993) in Bezug auf diejenigen Prozessmerkmale, bei denen sich Unterschiede zeigten, weitgehend überein. Von den 19 Prozessmerkmalen, die sich hier als charakteristischer für die implementierte psychodynamische Therapie erwiesen haben, waren 12 dies auch bei Jones und Pulos (1993; Q 6, Q 9, Q 28, Q 36, Q 49, Q 50, Q 67, Q 81, Q 83, Q 87, Q 93 und Q 98). Dies gilt auch für acht weitere Prozessmerkmale (Q 27, Q 17, Q 37, Q 57, Q 38, Q 30, Q 45 und Q 85) in Bezug auf die hier angewendete kognitiv-behaviorale Therapie. Weiterhin gehören 15 dieser 20 Merkmale des Therapieprozesses auch zu den nach Ablon und Jones (1998) für eine psychodynamische (Q 93, Q 36, Q 6, Q 67, Q 98 und Q 50) bzw. kognitiv-behaviorale (Q 38, Q 30, Q 85, Q 17, Q 45, Q 27, Q 86, Q 37 und Q 57) Therapie prototypischen Prozessmerkmalen. Diese können damit als zuverlässige prototypische Aspekte eines psychodynamischen bzw. kognitiv-behavioralen Behandlungsprozesses gelten, da sie nicht nur theoretisch zu den prototypischen Aspekten gehören, sondern auch in der Praxis (d.h. sowohl in der vorliegenden Studie als auch in der von Jones \& Pulos, 1993) relevanter für die psychodynamische bzw. die kognitiv-behaviorale Therapie waren.

Blagys und Hilsenroth (2002; vgl. 3.6 in Kapitel II) hatten geschlussfolgert, dass kognitivbehavioral orientierte Therapeuten im Vergleich zu psychodynamisch orientierten Therapeuten häufiger über extratherapeutische Aktivitäten sprechen, mehr Informationen geben, stärker in den Sitzungen strukturieren und Kognitionen stärker fokussieren. Diese Annahmen konnten auch in der vorliegenden Untersuchung bestätigt werden. Weiterhin gehen Blagys und Hilsenroth (2002) davon aus, dass Verhaltenstherapeuten künftige Erfahrungen stärker fokussieren und stärker spezifische Coping-Strategien vermitteln. Diese Annahmen konnten hier nicht überprüft werden, weil diese Prozessmerkmale im PQS nicht operationalisiert sind. 


\section{5. Übereinstimmung der implementierten Therapien mit ihren jeweiligen Prototypen}

Zur Beurteilung des Ausmaßes, in dem sich die hier durchgeführten Therapien an ihrer jeweiligen Therapietheorie orientieren, wurden Zusammenhänge zwischen den implementierten Therapien und einem kognitiv-behavioralen und einem psychodynamischen Prototyp (vgl. Ablon \& Jones, 1998) bestimmt. Diese Prototypen sollen alle, für eine ideal verlaufende psychodynamisch bzw. kognitiv-behavioral orientierte Therapiesitzung relevanten Prozessaspekte berücksichtigen. Die Bestimmung des Zusammenhangs zwischen den Prozessbeurteilungen der hier vorliegenden Therapiesitzungen der Patienten mit GAS und den Prototypen ermöglicht eine Aussage darüber, wie sehr die a priori als „psychodynamische“ bzw. als „,kognitiv-behaviorale“ Psychotherapie bezeichnete Therapieform tatsächlich mit ihrer jeweiligen Therapiekonzeption übereinstimmt. Erwartet wurde, dass das hier angewendete psychodynamische Therapieprogramm zur Behandlung der GAS stärker mit dem Prototyp einer psychodynamischen als mit dem einer kognitivbehavioralen Therapie zusammenhängt. Entsprechend wurde auch erwartet, dass das hier angewendete kognitiv-behaviorale Therapieprogramm zur Behandlung der GAS stärker mit dem Prototyp einer kognitiv-behavioralen als mit dem einer psychodynamischen Therapie zusammenhängt.

Zunächst zeigt sich, dass die Therapieprozessbeurteilungen der Patienten aus der gesamten Stichprobe deutlich höher mit dem kognitiv-behavioralen (.55) als mit dem psychodynamischen Prototyp (-.06) zusammenhängen. Dies bedeutet, dass die hier durchgeführte Psychotherapie zur Behandlung der GAS, unabhängig vom konkreten Therapieprogramm, insgesamt dem Ideal einer kognitiven Verhaltenstherapie stärker ähnelt als dem einer psychodynamischen Therapie.

Auch zeigt sich ein ähnlich überraschendes Ergebnis, wenn ausschließlich die psychodynamisch behandelte Stichprobe betrachtet wird. In dieser Stichprobe gibt es keine bedeutsamen Unterschiede zwischen der Übereinstimmung mit dem psychodynamischen Prototyp und der mit dem kognitiv-behavioralen Prototyp. Auch ist der Zusammenhang mit dem kognitiv-behavioralen Prototyp höher als der mit dem psychodynamischen Prototyp (.44 vs. .00). Dies bedeutet, dass in der hier durchgeführten psychodynamischen Therapie keine prototypischen Elemente einer psychodynamischen Therapie und einige, aber nicht signifikant mehr Elemente einer kognitiv-behavioralen Therapie angewendet worden sind. Die Vermutung, nach der die hier implementierte spezielle Form einer psychodynamischen 
Therapie dem psychodynamischen Prototyp ähnelt, ließ sich also nicht bestätigen. Mangelnde Reliabilität der Therapieprozessmessungen oder des Messinstruments als Erklärung für diesen überraschenden Befund ist aufgrund der berichteten Daten weitgehend auszuschließen. Auch mangelnde Manualtreue durch die Therapeuten als Ursache dieses Befunds scheint zunächst eher unwahrscheinlich, da diese hier durch vorhergehendes Training in der Anwendung des Manuals und durch regelmäßige Supervision gewährleistet wurde. Allerdings wurde sie eben nicht explizit gemessen. Schon Ablon und Jones (2002) hatten gezeigt, dass eine implementierte Therapie trotz Überprüfung der Manualtreue nicht unbedingt ihrem Manual entsprechen muss. Weiterhin können auch keine Aussagen über die „,competence“, d.h. über die kompetente Anwendung des Manuals (vgl. Waltz, Addis, Koerner \& Jacobson, 1993) getroffen werden, da diese hier ebenfalls nicht erfasst wurde. Dies könnte bedeuten, dass sich die Therapeuten zwar an das Manual gehalten haben, dass sie dieses aber nicht kompetent umgesetzt haben. So gehen beispielsweise Ablon et al. (2006) davon aus, dass Therapeuten gerade in kurzen psychodynamischen Therapien ganz verschiedene Interventionen nutzen. Darunter können auch andere als typisch psychodynamische, wie z.B. kognitiv-behaviorale sein. Denkbar ist auch, das die psychodynamischen Therapeuten bei den vorliegenden Behandlungen strukturierter und aktiver waren als es sonst in psychodynamischen Therapien üblich ist, weil die Therapien zeitlich begrenzt waren und sie im Rahmen der Studie möglicherweise besonders bemüht waren, deutliche Verbesserungen im Patienten zu erzielen. Zwar sind die psychodynamischen Therapeuten insgesamt deutlich weniger strukturiert und aktiv als die kognitiv-behavioralen Therapeuten, dennoch liegen sie bei diesem Prozessmerkmal auch eher im oberen Bereich der Skala $(\mathrm{M}=5,78, \mathrm{sd}=1,36)$.

Weiterhin kann neben der mangelnden Manualtreue oder Kompetenz der Therapeuten eine weitere Erklärung für die vorliegenden Befunde darin gesehen werden, dass entweder das hier angewendete psychodynamische Therapieprogramm eben nicht typisch für psychodynamische Therapien ist oder, dass der psychodynamische Prototyp von Ablon und Jones (1998) doch nicht alle prototypischen Aspekte einer solchen erfassen konnte.

Für die hier angewendete kognitiv-behaviorale Therapie hingegen zeigen sich die erwarteten Ergebnisse. Hier ist die Übereinstimmung mit dem kognitiv-behavioralen Prototyp deutlich höher als die mit dem psychodynamischen Prototyp. Aus diesem Befund ist zu schlussfolgern, dass zumindest die hier implementierte kognitiv-behaviorale Therapie tatsächlich theoriekonform konzipiert wurde. Dies impliziert dann aber auch, dass im 
kognitiv-behavioralen Prototyp alle tatsächlich relevanten Therapieprozessaspekte berücksichtigt wurden.

Aufgrund der Tatsache, dass sich die zur Prototypenbildung befragten Therapie-Experten jeweils gleichermaßen einig waren in ihrer Vorstellung eines prototypischen psychodynamischen bzw. kognitiv-behavioralen Behandlungsprozesses (vgl. Ablon \& Jones, 1998), ist von der Validität der beiden Prototypen auszugehen. Die fehlende Orientierung der implementierten psychodynamischen Therapie am psychodynamischen Prototyp wäre in diesem Fall dann also weniger durch die mangelhafte Validität des psychodynamischen Prototyps als vielmehr durch die Konzeptionierung der hier implementierten psychodynamischen Therapie zu erklären.

$\mathrm{Ob}$ die hier vorliegenden PQS-Prozessbeurteilungen so getroffen wurden, wie es vom Entwickler des PQS intendiert war, muss offen bleiben, da die Rater kein PQS-Training durch seinen Entwickler oder einen Mitarbeiter aus seiner Arbeitsgruppe erhielten. Zwar existiert ein ausführliches Manual zur Anwendung dieses Instruments, allerdings lässt dieses manchmal mehr als nur eine Möglichkeit zur Beurteilung des jeweiligen Aspekts des Therapieprozesses zu. So ist denkbar, dass die Rater zwar untereinander hoch in ihren Prozessbeurteilungen übereinstimmten (hohe Reliabilität), das PQS jedoch dennoch anders anwendeten als es von Jones selbst intendiert war (Validität).

Eine weitere denkbare Erklärung für die vorliegenden Befunde ist auch in der kleinen Stichprobengröße innerhalb der beiden Therapiegruppen zu sehen. Je kleiner die Stichprobe ist, umso geringer ist auch die Power der Tests, d.h. umso unwahrscheinlicher ist es, Effekte in der Stichprobe zu finden. Daher ist es schwierig zu entscheiden, ob die nicht signifikanten Zusammenhänge dadurch zustande kommen, dass tatsächlich kein Effekt da ist oder eben durch die kleine Stichprobe.

Insgesamt kann also nur festgehalten werden, dass zwischen der hier angewendeten kognitiv-behavioralen Therapie und dem kognitiv-behavioralen Prototyp ein erwartungsgemäßer Zusammenhang besteht, zwischen der hier angewendeten psychodynamischen Therapie und dem psychodynamischen Prototyp jedoch nicht. Mögliche Gründe dafür wurden diskutiert, allerdings ist eine abschließende Beurteilung aufgrund der vorliegenden Daten derzeit nicht möglich. 


\section{Zusammenhang zwischen der Orientierung an einem Prototyp und dem}

\section{Outcome}

Um Aussagen darüber treffen zu können, ob die Orientierung an einem der beiden Prototypen mit einer bedeutsamen Verbesserung des Patienten durch die Therapie zusammenhängt, wurden Korrelationen zwischen der Orientierung am psychodynamischen bzw. kognitiv-behavioralen Prototyp und den Therapieerfolgsmaßen bestimmt. Daneben wurde auch der Einfluss, den die Orientierung am jeweils eigenen Prototyp auf das Therapieergebnis für alle hier behandelten Patienten gemeinsam hat, untersucht. Als Therapieerfolgsmaße wurden zur Erfassung der Angst bzw. Ängstlichkeit die Hamilton Angst Skala (Hamilton, 1976), das Beck Angst Inventar (Margraf \& Ehlers, 2003), das State Trait Angst Inventar (Laux et al., 2002) sowie der Penn State Worry Questionnaire (Stöber \& Bittencourt, 2002) gewählt. Zur Erfassung der Depressivität wurden die Hamilton Depression Skala (Hamilton, 1986) und das Beck Depression Inventar (Hautzinger, 2002a) erhoben. Weiterhin wurde zur Erfassung interpersonaler Schwierigkeiten das Inventar Interpersonaler Probleme (Horowitz et al., 2002) eingesetzt. Hier wurden keine expliziten Hypothesen formuliert.

Für alle Patienten zeigt sich, dass die Orientierung am jeweils eigenen Prototyp zu einer bedeutsamen Abnahme des ,worrying“-Verhaltens der Patienten führt (im Penn State Worry Questionnaire), d.h. zu einer signifikanten Reduktion des Ausmaßes an pathologischer Besorgnis. Sonst zeigte sich für kein weiteres Therapieerfolgsmaß ein bedeutsamer Zusammenhang mit der Orientierung am jeweils eigenen Prototyp. Aus diesen Befunden ist zu schlussfolgern, dass es in den Therapien gelungen ist, die für die GAS zentrale pathologische Besorgnis zu reduzieren und zwar dann, wenn sich die Therapeuten jeweils an ihrem eigenen Therapiemanual orientiert haben.

Werden ausschließlich die kognitiv-behavioral behandelten Patienten betrachtet, findet sich ein widersprechender Befund. Hier zeigt sich ein positiver Zusammenhang zwischen der Orientierung der Therapie am psychodynamischen Prototyp und dem Therapieerfolg im Penn State Worry Questionnaire. Je mehr typisch psychodynamische zusätzlich zu den gemäß Therapiemanual kognitiv-behavioralen Interventionen demnach angewendet werden, umso größere Veränderungen werden mit der Therapie erreicht, d.h. umso seltener und weniger intensiv sorgt sich der Patient am Ende der Therapie. Ein ebenso überraschendes Ergebnis findet sich auch für den Zusammenhang mit dem Therapieerfolg im Beck Depression Inventar. Je mehr typisch kognitiv-behaviorale Prozessmerkmale in der kognitiv-behavioralen 
Therapie auftreten, umso geringer fällt der Therapieerfolg aus, d.h. umso mehr depressive Gedanken und Verhaltensweisen behält der Patient auch noch nach seiner Therapie. Diese widersprüchlichen Befunde stehen sicherlich erneut mit den kleinen Stichprobengrößen innerhalb der beiden Therapieansätze im Zusammenhang.

Bei den psychodynamisch behandelten Patienten zeigt sich weder für die Orientierung am psychodynamischen noch für die Orientierung am kognitiv-behavioralen Prototyp ein bedeutsamer Zusammenhang mit dem Outcome. In einer vorangegangenen Untersuchung (Winkelbach, 2007) hat sich jedoch gezeigt, dass die hier implementierte psychodynamische Therapie zur Behandlung der Generalisierten Angststörung effektiv ist. Die in der vorliegenden Studie fehlenden Zusammenhänge zwischen der Orientierung am Prototyp und dem Therapieerfolg lassen sich deshalb nicht mit der fehlenden oder mangelhaften Wirksamkeit der psychodynamischen Psychotherapie erklären. Die Wirkfaktoren dieser spezifischen psychodynamischen Therapie der GAS konnten demnach durch den Prototyp einer psychodynamischen Therapie nach Ablon und Jones (1998) nicht erfasst werden. Auch in diesem Fall konnte in der vorliegenden Untersuchung also keine Übereinstimmung zwischen Theorie und Praxis gefunden werden (vgl. Ablon et al., 2006). Dies kann an der Konstruktionsweise des PQS selbst liegen, da dieses weitgehend frei von theoretischen Konzepten entwickelt wurde und daher evtl. den Therapieprozess der hier implementierten psychodynamischen Therapie nicht spezifisch genug erfassen kann. Dann sollten die im PQS erfassten Prozessmerkmale weiter spezifiziert und spezifische Merkmale der unterschiedlichen psychodynamischen Behandlungsansätze berücksichtigt werden. Ablon und Jones (1998) selbst hatten ebenfalls keine Zusammenhänge mit der Orientierung am psychodynamischen Prototyp und einem positiven Outcome in einer psychodynamischen Psychotherapie von Patienten mit einer Posttraumatischen Belastungsstörung gefunden. Auch sie schlagen deshalb vor, einen spezifischen Prototyp für solche supportiven psychodynamischen Therapien zu entwickeln. Möglicherweise hätten sich mit einem solchen dann auch für die vorliegende supportiv-expressive Therapie der GAS Zusammenhänge mit dem Therapieerfolg finden lassen.

Jones und Pulos (1993) und Ablon und Jones (1998) fanden, dass eine kognitivbehaviorale Therapie umso erfolgreicher war, je mehr psychodynamische Techniken die Therapeuten einsetzten. In der psychodynamisch behandelten Stichprobe gab es keine eindeutigen Zusammenhänge mit dem Outcome, weder für die Orientierung am psychodynamischen noch die am kognitiv-behavioralen Prototyp. Dies bedeutet, dass, wenn 


\section{Literaturverzeichnis}

die kognitiv-behavioral orientierten Therapeuten auch psychodynamische Interventionen anwenden, genau diese positiv mit dem Outcome korrelieren. Dies deutet auf die Wichtigkeit von „eklektischem“, adaptivem Arbeiten hin.

Die vorliegenden Ergebnisse zeigen, wie wichtig die genaue Untersuchung des Therapieprozesses ist, da die bloße Bezeichnung eines bestimmten Therapieansatzes noch nichts darüber aussagt, was tatsächlich in der Therapie umgesetzt wird und was hilfreich ist. Ablon und Jones (2002) vermuten, dass sich zwar die Terminologie und die Art, psychologische Konstrukte und Prozesse innerhalb der Therapie zu definieren, zwischen verschiedenen Therapieformen unterscheidet, dass aber ähnliche inhaltliche Überlegungen dahinter stehen und es deswegen zu Überschneidungen zwischen theoretisch unterschiedlichen Therapieprozessen kommt.

\section{Therapieprozessmerkmale als Prädiktoren des Outcomes}

Mit Hilfe dieser explorativen Fragestellung soll untersucht werden, ob es im Therapieprozess Prädiktoren des Therapieerfolgs gibt. Der Therapieerfolg wurde auch hier gemessen über die Reduktion der Angst bzw. der Sorgen (mit Hilfe des Beck Angst Inventars, der Hamilton Angst Skala, dem Penn State Worry Questionnaire und dem State Trait Angst Inventar) sowie der Depressivität (mit Hilfe des Beck Depression Inventars und der Hamilton Depression Skala). Außerdem wurde er an der Reduktion interpersonaler Probleme (gemessen mit dem Inventar Interpersonaler Probleme) festgemacht.

In der gesamten Stichprobe ließen sich im Therapieprozess für keines der sieben Therapieerfolgsmaße bedeutsame Prädiktoren des Therapieerfolgs identifizieren. Keines der mit dem Psychotherapie Prozess Q-Sort erfassten Merkmale des Therapieprozesses vermag demnach die Ergebnisse der Therapien vorherzusagen.

In der psychodynamisch behandelten Therapiegruppe erwies sich ein Merkmal des Therapieprozesses als bedeutsam für die Vorhersage des Therapieerfolgs. Je weniger in der psychodynamischen Therapie über Träume und Phantasien des Patienten gesprochen wird, umso weniger ,,Worrying“ zeigt der Patient am Ende der Therapie (gemessen mit dem Penn State Worry Questionnaire). Immerhin 41 \% der Varianz in Bezug auf das Therapieergebnis kann mit Hilfe dieses Prozessmerkmals erklärt werden. Dieser Befund bedeutet, dass es gerade auch in der hier angewendeten psychodynamischen Therapie wichtig ist, an realen Themen zu arbeiten, da dies einen sorgenreduzierenden Effekt zeigt. Für alle weiteren Maße 


\section{Literaturverzeichnis}

zur Erfassung des Therapieerfolgs haben die untersuchten Prozessmerkmale keine bedeutsame Vorhersagekraft.

Auch in der hier implementierten kognitiv-behavioralen Therapie konnte nur ein einziger Prädiktor des Therapieerfolgs identifiziert werden. Je mehr der Therapeut die Aufmerksamkeit auf das nonverbale Verhalten des Patienten lenkt, umso weniger Angst zeigt der Patient am Ende der Therapie (gemessen mit dem Beck Angst Inventar). $29 \%$ der Varianz in Bezug auf den Therapieerfolg können mit diesem Therapieprozessmerkmal erklärt werden. Einer der Gründe, weswegen Patienten mit GAS so selten eine Therapie aufnehmen, ist, dass sie sich über das pathologische Ausmaß ihrer Sorgen nicht bewusst sind. Der Therapeut weist den Patienten also evtl. auf ihm zunächst nicht zugängliche weitere Angstbereiche hin, was eine erfolgreiche Bearbeitung dieser Themen in der Therapie überhaupt erst ermöglicht.

Auch wenn die jeweils charakteristischsten Merkmale des Therapieprozesses gemeinsam betrachtet werden, können diese den Therapieerfolg nicht bedeutsam vorhersagen.

Eine bedeutsame Vorhersagekraft weisen insgesamt also nur zwei Merkmale des Therapieprozesses auf. Diese Vorhersagekraft zeigen sie bei zwei Therapieerfolgsmaßen, die gezielt die Hauptproblematik der Patienten, d.h. die Angst bzw. die Sorgen, erfassen. Diese beiden Merkmale beziehen sich jeweils auf Interventionen des Therapeuten bzw. in der Sitzung fokussierte Themen, die eher untypisch für die jeweilige Therapieform sind. So ist es in der kognitiv-behavioralen Therapie eher nicht typisch, dass der Therapeut auf das nonverbale Verhalten des Patienten hinweist. Es zeigt sich jedoch, dass sich diese Intervention positiv auf den Therapieerfolg auswirkt. Ebenso ist es eher typisch für psychodynamische Therapien, dass auch Träume und Phantasien berücksichtigt werden. Hier zeigt sich jedoch, dass der Therapieerfolg umso höher ist, je weniger der Therapeut dies tut. Diese beiden Befunde deuten darauf hin, dass therapeutische Interventionen, die vom theoretischen Konzept der Therapie abweichen, durchaus positive Effekte auf die Therapie ausüben können. Offensichtlich sind außerdem die typischen Prozessaspekte nicht unbedingt diejenigen, die den Therapieerfolg am besten vorhersagen können. Eine „reine“ Umsetzung einer Therapietheorie in die Praxis scheint also mehr Theorie als tatsächlicher Alltag zu sein.

Insgesamt haben die hier erfassten Prozessmerkmale nur eine kleine Vorhersagekraft für den Erfolg der Therapien. Da die beiden hier angewendeten Therapieprogramme jedoch signifikant positive Effekte auf die Symptome der Patienten hatten (Winkelbach, 2007), konnten die Wirkfaktoren dieser Therapien mit dem hier verwendeten Messinstrument 


\section{Literaturverzeichnis}

offensichtlich nicht erfasst werden. Wirkfaktoren sind als Bindeglieder zwischen therapeutischen Interventionen und Veränderungen im Erleben und Verhalten des Patienten zu sehen. Wie Ablon und Jones (1999) betonen, zeigen diese jedoch keine lineare Wirkung. Vielmehr entfalten sie ihre Wirkung erst in einem komplexen Gefüge aus verschiedenen Prozessmerkmalen, die in einer Interaktion zueinander stehen. Durch die spezielle Konstruktion des Psychotherapie Prozess Q-Sort, nach dem alle Prozessmerkmale, die hoch miteinander korrelierten, eliminiert wurden, sind solche Interaktionen nicht zu erfassen. Gerade für therapeutische Techniken gilt jedoch, dass sie nicht unabhängig vom Kontext immer die gleiche Wirkung haben. Daher können solche Interventionen einzeln den Therapieerfolg nicht vorhersagen (vgl. Ablon \& Jones, 1999). Solche komplexen Interaktionen sind außerdem durch die relativ einfachen Auswertungsmechanismen, die zudem die Effekte über Patienten-Therapeuten-Paare mitteln, nicht zu erfassen. Hier sollten komplexere Auswertungsstrategien, die zwischen einem Gruppen- und Einzelfalldesign variieren, herangezogen werden, weil sich nur so der reziproke Einfluss in der Interaktion zwischen verschiedenen Prozessmerkmalen erfassen ließe (vgl. Jones \& Price, 1998; Pole \& Jones, 1998).

\section{Grenzen der Untersuchung}

Wie die vorangegangenen Abschnitte zeigen, sind einige der Befunde aus der vorliegenden Studie widersprüchlich. Ein Grund für diese z.T. überraschenden und auch inkonsistenten Ergebnisse ist sicherlich in der kleinen Stichprobe zu sehen. So gab es nur 19 Patienten, die mit dem hier angewendeten psychodynamischen Therapieprogramm und nur 23 Patienten, die mit dem hier angewendeten kognitiv-behavioralen Therapieprogramm behandelt wurden. In kleinen Stichproben jedoch ist die Teststärke vergleichsweise gering und würde durch eine Bonferroni-Adjustierung noch geringer werden. Deswegen wurde auf eine solche verzichtet, was dann aber zu der schon diskutierten $\alpha$ - Kumulierung führen kann. Möglicherweise liegen damit hier also Befunde vor, die für die Population gar nicht gelten.

Auch die hier nicht explizit erfasste Manualtreue der Therapeuten bzw. die kompetente Umsetzung des Manuals durch die Therapeuten (vgl. Waltz et al., 1993) ist gerade im Zusammenhang mit der Interpretation der Ergebnisse in Bezug auf die Orientierung am Prototyp problematisch. So behandelten die Therapeuten zwar zunächst einige Trainingsfälle und wurden bei der Behandlung der Studienpatienten supervidiert, dennoch ist es nicht möglich, daraus eindeutig auf die Manualtreue der Therapeuten bzw. auf die kompetente 


\section{Literaturverzeichnis}

Umsetzung dieses zu schließen. Dies wiederum erschwert die Interpretation der Befunde, nach denen sich zumindest die psychodynamischen Therapeuten eben nicht an ihrem Prototyp orientiert haben. Die Frage, ob dies an mangelnder Manualtreue bzw. Kompetenz oder an der fehlenden Übereinstimmung des Manuals mit seinem Prototyp liegt, muss daher vorläufig unbeantwortet bleiben.

Eine Schwäche der vorliegenden Studie kann auch im hier verwendeten Instrument zur Erfassung des Psychotherapieprozesses liegen. Dieses ist für den amerikanischen Sprachraum entwickelt und aus dem Englischen ins Deutsche übersetzt worden (vgl. Albani et al., 2000). Ob diese Adaption und die Adaption der Prototypen ohne Probleme möglich ist, ist ohne weitere Untersuchungen nicht zu sagen. Möglicherweise sind die vergleichsweise wenigen Befunde zum Zusammenhang der Orientierung am Prototyp mit dem Outcome auch darauf zurückzuführen. Daher sollten auch deutsche Pendants zu den amerikanischen Prototypen entwickelt werden.

\section{Einordnung der Ergebnisse und Ausblick}

Die Therapieprozessbeurteilungen, die im Rahmen der vorliegenden Arbeit mittels des Psychotherapie Prozess Q-Sort erhoben wurden, erwiesen sich insgesamt als sehr reliabel. Erwartungsgemäß zeigten sich zwischen den beiden Messzeitpunkten (10. und 18. Sitzung) keine Unterschiede. Ebenfalls keine bzw. kaum bedeutsame Unterschiede zwischen den Therapieprozessen einer psychodynamischen und einer kognitiv-behavioralen Therapie gab es in Bezug auf die Prozessvariablen, die den emotionalen Zustand des Patienten bzw. sein Verhalten in der Therapie und die Interaktion zwischen Patient und Therapeut bzw. die in der Sitzung thematisierten Inhalte beschreiben. Hier unterschieden sich 70 von 100 Prozessmerkmale nicht voneinander. Die verbleibenden 30 bedeutsamen Unterschiede bezogen sich fast ausschließlich auf die Prozessvariablen, die therapeutische Haltungen und Interventionen beschreiben. Dieser Befund, dass sich in der Mehrzahl der untersuchten Prozessvariablen keine Unterschiede zwischen den beiden Therapieprozessen zeigte, wird darauf zurückgeführt, dass hier eine homogene Patientengruppe behandelt und untersucht wurde, die sich auch im Verhalten in der Therapie ähnelt.

Um die Ergebnisse im Hinblick auf die hier gefundenen Unterschiede zwischen den beiden Therapieprozessen einordnen zu können, ist es notwendig ihre interne und externe Validität zu beurteilen. Unter interner Validität wird verstanden, inwiefern die in der vorliegenden Studie gefundenen Unterschiede auf tatsächliche Unterschiede in den beiden 


\section{Literaturverzeichnis}

Therapieprozessen zurückzuführen sind. Unter externer Validität wird die Generalisierbarkeit bzw. Verallgemeinerbarkeit der Befunde auch auf andere Stichproben verstanden.

Zur Frage der internen Validität der Ergebnisse ist zunächst zu überlegen, inwiefern das PQS durch seine Itemauswahl zu einer Überhöhung der wenigen gefundenen Unterschiede zwischen den beiden Therapieprozessen beigetragen haben könnte. Aufgrund der Vorgehensweise bei der Konstruktion und aufgrund der bereits berichteten Befunde aus Untersuchungen zur Validität der Skala ist dies jedoch unwahrscheinlich. Weiterhin könnten implizite Theorien und damit verbundene globale Inferenzschlüsse der Rater die gefundenen Unterschiede bedingen. Da die Beurteiler zwar Hintergrundwissen zu den beiden Therapierichtungen, jedoch keine vertiefte Ausbildung in einem der beiden Therapieansätze hatten, und außerdem das PQS weitgehend theoriefrei konstruiert wurde, d.h. ohne Verwendung von Begrifflichkeiten oder Konzepten, die eindeutig einem der beiden untersuchten Therapieverfahren zuzuordnen wären, ist dies eher unwahrscheinlich. Die Entscheidung für die Wahl von unerfahrenen Ratern hat jedoch den Nachteil, dass diese bestimmte komplexe Interventionen möglicherweise nicht erkennen. Dies würde zu einer Unterschätzung der Unterschiede führen. Diesem Bias wurde durch ein umfangreiches Ratertraining sowie durch das ausführliche Manual jedoch entgegengewirkt. Denkbar wäre auch, dass die gefundenen Unterschiede im Hinblick auf die beiden Therapieprozesse durch systematische Unterschiede zwischen den psychodynamisch und kognitiv-behavioral orientierten Therapeuten (ausgenommen von ihrer theoretischer Orientierung) begründet sind. Dies scheint jedoch nicht wahrscheinlich, da sie sich im Hinblick auf Alter und durchschnittliche Berufserfahrung nicht voneinander unterscheiden.

Zumindest im Hinblick auf die hier genannten Aspekte, die die interne Validität dieser Untersuchung einschränken könnten, kann von intern validen Unterschieden ausgegangen werden. Dafür spricht auch, dass sich die beiden Patientengruppen aufgrund der randomisierten Zuteilung der Patienten zu den beiden Therapiegruppen bis auf bei zwei soziodemographischen Merkmalen nicht bedeutsam voneinander unterscheiden. Die Patienten in der kognitiv-behavioral behandelten Gruppe arbeiteten häufiger in einer Vollzeitstelle. Die psychodynamisch behandelten Patienten hatten häufiger eine feste Partnerschaft. Allerdings ist nicht davon auszugehen, dass sich das Verhalten der Patienten in der Therapie durch diese Unterschiede voneinander unterscheidet und dass die gefundenen Unterschiede im Therapieprozess darauf basieren. In den relevanten Variablen wie z.B. psychotherapeutische oder psychiatrische Vorbehandlung oder Bildungsniveau unterscheiden sie sich jedoch nicht. 


\section{Literaturverzeichnis}

Im Hinblick auf die externe Validität ist zunächst die Frage nach der Reaktivität der Messung, d.h. inwiefern die analysierte Therapiesitzung durch den Messvorgang selbst beeinflusst wurde, zu stellen. Das Tonbandgerät, mit dem die Sitzungen aufgezeichnet wurden, kann ein Störfaktor gewesen sein, der den Ablauf und den Inhalt der Sitzungen verändert haben könnte. So ist denkbar, dass die Patienten sich dem Therapeuten deshalb weniger geöffnet und die Therapeuten ihr eigenes Verhalten gezielt verändert haben, indem sie verstärkt Interventionen einsetzten, die sie für typisch für ihre eigene theoretische Orientierung hielten. Dies ist jedoch unwahrscheinlich, da eine Tonbandaufzeichnung die Sitzungen vermutlich weniger stört als bspw. eine Videoaufzeichnung. Außerdem wurden Sitzungen aus der mittleren Therapiephase untersucht, in denen bereits eine Gewöhnung an das Procedere innerhalb der Sitzungen sowohl bei den Patienten als auch bei den Therapeuten eingetreten sein sollte. Auch sind solche Reaktivitätseffekte nur dann ein Problem, wenn von einer Interaktion zwischen Reaktivität und Therapierichtung auszugehen ist, d.h. wenn diese in einer der beiden Therapien stärker ist als in der anderen. Jedoch gibt es keine Veranlassung, dies für die vorliegende Untersuchung anzunehmen. Auch von der Repräsentativität der untersuchten Stichprobe für die Population der Betroffenen mit einer Generalisierten Angststörung ist aufgrund der vielfältigen Wege zur Rekrutierung der Patienten auszugehen. Die externe Validität der Ergebnisse scheint zumindest in Bezug auf die genannten Aspekte demnach nicht gefährdet zu sein.

Validitätseinschränkungen sind jedoch durch die kleine Stichprobengröße (s.o.) durchaus denkbar. Für die Validität der Befunde insgesamt spricht jedoch, dass die vorliegenden Ergebnisse mit denen vorangegangener Untersuchungen weitgehend übereinstimmen.

Trotz einiger überraschender Befunde in der vorliegenden Studie wurde mit dieser Arbeit ein weiterer Schritt im Hinblick auf die Kenntnis der Abläufe innerhalb eines Therapieprozesses unternommen. So zeigt sich, dass sich die Therapeuten in dieser Studie, unabhängig von ihrer eigenen theoretischen Orientierung, eher an einem kognitivbehavioralen Modell einer Psychotherapie orientierten. Allerdings zeigten sich insgesamt gar keine oder keine eindeutigen Zusammenhänge zwischen der Orientierung an diesem Prototyp und einem positiven Outcome der Therapie. Das gleiche gilt für den Zusammenhang der Orientierung am psychodynamischen Prototyp mit dem Therapieerfolg. Dafür fand sich ein Zusammenhang zwischen der Orientierung am jeweils eigenen Prototyp und einem reduzierten Ausmaß an pathologischer Besorgnis. Des Weiteren konnten mit der vorliegenden Studie die bisher existierenden Befunde bzgl. der Therapieprozessmerkmale als Prädiktoren 


\section{Literaturverzeichnis}

des Therapieerfolgs nicht bestätigt werden, zumal sich insgesamt nur zwei Merkmale des Therapieprozesses als Prädiktoren erwiesen. Im Hinblick auf (spezifische und unspezifische) Wirkfaktoren einer psychodynamischen und einer kognitiv-behavioralen Psychotherapie konnten demnach keine eindeutigen Befunde erbracht werden.

Idealerweise sollten Prozessanalysen als begleitende Untersuchung zum Standard von Therapie-Outcome-Studien gehören. Denn nur mit deren Hilfe ist es möglich, Veränderungsmechanismen psychotherapeutischer Behandlungen besser zu verstehen und dann auch genauere Aussagen über das Outcome, gerade auch im Hinblick auf das Äquivalenzparadoxon, zu treffen.

Mit Hilfe des hier verwendeten Instruments zur Erfassung des Therapieprozesses, dem PQS, ist es nicht möglich, globale Aussagen über den Therapieprozess zu formulieren. Aufgrund der Konstruktionsweise des PQS sind Aussagen über einzelne Merkmale des Therapieprozesses möglich, allerdings lassen sich diese eben nicht zu Subskalen und damit zu globaleren Aussagen zusammenfassen. Alle Merkmale des Therapieprozesses werden jeweils nur über ein Item erfasst. So sind einerseits keine Aussagen über komplexe Interaktionsmuster und anderseits auch keine Aussagen über den Kontext, in dem dieses Prozessmerkmal auftritt, möglich. Beide Vorgehensweisen- die des PQS sowie die über verschiedene Subskalen- haben Vor- und auch Nachteile. Das PQS z.B. ermöglicht es, anders als globalere Messinstrumente, den Therapieprozess genau zu untersuchen und detaillierte Aussagen über einzelne Merkmale des Prozesses der hier durchgeführten Therapien zu formulieren. Für weitere Untersuchungen empfiehlt es sich, neben dem PQS ein weiteres zusätzliches Instrument einzusetzen, das Aussagen über übergeordnete Therapieprozessaspekte (in Form von Faktoren oder Subskalen) ermöglicht. Hier bietet sich z.B. die „Comparative Psychotherapy Process Scale” (CPPS; Hilsenroth, Blagys, Ackerman, Bonge \& Blais, 2005) an. Die CPPS besteht aus 20 Items und ist damit wesentlich schneller zu bearbeiten als das PQS mit seinen 100 Items. Diese 20 Items werden auf einer siebenstufigen Likert-Skala von, $0=$ überhaupt nicht charakteristisch“ bis , $6=$ extrem charakteristisch“ beurteilt und lassen sich zu zwei Subskalen zusammenfassen. Dies sind die beiden Subskalen ,psychodynamisch-interpersonale Techniken“ und „kognitiv-behaviorale Techniken“. Einige der Items aus der CPPS stimmen mit denen des PQS überein. Würden sowohl das PQS als auch die CPPS eingesetzt, wären sowohl Aussagen über den Einfluss von übergeordneten Subskalen als auch Aussagen über deskriptive Phänomene des Therapieprozesses möglich. Die Vor- und Nachteile der beiden Vorgehensweisen könnten 


\section{Literaturverzeichnis}

daher am besten durch eine Integration beider Vorgehensweisen genutzt bzw. vermieden werden.

Weitere Untersuchungen an größeren und vergleichbaren Stichproben wären sinnvoll, um die vorliegenden Befunde besser einordnen und relevante Therapieprozessfaktoren noch besser kategorisieren zu können. Dabei sollte die Erfassung der Manualtreue sowie dessen kompetente Umsetzung im Rahmen von komparativer Therapie-Outcome- und auch von Therapieprozessforschung zu einem festen Bestandteil des Studiendesigns werden, da nur bei vorliegender Manualtreue und kompetenter Nutzung dieses eindeutige Rückschlüsse auf die zugrunde liegenden Therapiekonzepte gezogen werden können. In Bezug auf die vorliegende Untersuchung könnte die nicht explizit erfasste Manualtreue sowie die kompetente Umsetzung des Manuals nachträglich untersucht werden. Damit wäre dann auch in Bezug auf die Zusammenhänge zwischen der Orientierung am Prototyp und dem Outcome eine eindeutigere Interpretation der Befunde möglich.

Die vorliegende Studie konnte erste Anhaltspunkte dafür liefern, dass auch im deutschen Sprachraum die Bezeichnung einer Psychotherapie noch nicht unbedingt etwas über ihre konkrete Umsetzung in die Praxis aussagt und dass die jeweils prototypischen Interventionen nicht unbedingt mit dem Outcome zusammenhängen müssen. Gerade für komparative Outcome-Untersuchungen ist Prozessforschung, so wie sie mit der vorliegenden Studie durchgeführt wurde, daher besonders wichtig. 


\section{Literaturverzeichnis}

\section{Literaturverzeichnis}

Ablon, J.S. \& Jones, E.E. (1998). How expert clinicians prototypes of an ideal treatment correlate with outcome in psychodynamic and cognitive-behavioral therapy. Psychotherapy Research, 8, 71-83.

Ablon, J.S. \& Jones, E.E. (1999). Psychotherapy Process in the National Institute of Mental Health Treatment of Depression Collaborative Research Program. Journal of Consulting and Clinical Psychology, 67, 64-75.

Ablon, J.S. \& Jones, E.E. (2002). Validity of controlled clinical trials of psychotherapy: findings from the NIMH Treatment of Depression Collaborative Research Program. American Journal of Psychiatry, 159, 775-783.

Ablon, J.S., Levy, R.A. \& Katzenstein, A. (2006). Beyond Brand Names of Psychotherapy: Identifying Empirically Supported Change Processes. Psychotherapy: Theory, Research, Practice, Training, 43, 216-231.

Ahn, H. \& Wampold, B.E. (2001). Where Oh where are the Specific Ingredients? A MetaAnalysis of Component Studies in Counseling and Psychotherapy. Journal of Counseling Psychology, 48, 251-257.

Albani, C. \& Blaser, G. (2005). PQS. Psychotherapy Process Q-Sort. In B. Strauß \& J. Schumacher (Hrsg.), Klinische Interviews und Ratingskalen (S. 337-340). Göttingen: Hogrefe.

Albani, C., Blaser, G., Jacobs, U., Jones, E.E., Geyer, M. \& Kächele, H. (2000). Die Methode des Psychotherapie-Prozeß Q-Sort. Zeitschrift für Klinische Psychologie und Psychotherapie, 48, 151-171.

Ambühl, H., Orlinsky, D., Cierpka, M. Buchheim P., Meyerberg, J. \& Willutzki, U. (1995). Zur Entwicklung der theoretischen Orientierung von PsychotherapeutInnen. Psychotherapie, Psychosomatik und Medizinische Psychologie, 45, 109-120.

American Psychiatric Association (1980). Diagnostic and Statistical Manual of Mental Disorders ( $3^{\text {rd }}$ ed.). Washington/DC: Author.

American Psychiatric Association (1987). Diagnostic and Statistical Manual of Mental Disorders ( $3^{\text {rd }}$ ed., rev.). Washington/DC: Author.

American Psychiatric Association (1994). Diagnostic and Statistical Manual of Mental Disorders ( $4^{\text {th }}$ ed.). Washington/ D.C: Author [dt. Übersetzung: Saß, H., Wittchen, H.U. \& Zaudig, M. (1996). Diagnostisches und Statistisches Manual psychischer Störungen (DSM-IV)]. Göttingen: Hogrefe. 
Andrews, G. \& Harvey, R. (1981). Does psychotherapy benefit neurotic patients? A reanalysis of the Smith, Glass \& Miller data. Archives of General Psychiatry, 38, 1203-1208.

Angst, J. \& Vollrath, M. (1991). The natural history of anxiety disorders. Acta Psychiatrica Scandinavica, 84, 446-452.

Ballenger, J.C., Davidson, J.R.T., Lecrubier, Y., Nutt, D.J., Borkovec, T.D., Rickels, K., Stein, D.J. \& Wittchen, H.-U. (2001). Consensus statement on Generalized Anxiety Disorder from the international consensus group on depression and anxiety. Journal of Clinical Psychiatry, 62 (suppl. 11), 53-58.

Bandura, A. (1969). Principles of behaviour modification. New York: Holt, Rinehart \& Winston.

Barkham, M., Rees, A., Shapiro, D.A., Stiles, W.B., Agnew, R.M., Halstead, J., Culverwell, A.L. \& Harrington, V.M.G. (1996). Outcomes of time-limited psychotherapy in applied settings: Replication of the second Sheffield Psychotherapy Project. Journal of Consulting and Clinical Psychology, 64, 1079-1085.

Barlow, D.H. (1988). Anxiety and its disorders: The nature and treatment of anxiety and panic. New York: Guilford.

Barlow, D.H. (1991). The nature of anxiety: Anxiety, depression, and emotional disorders. In R.M. Rapee \& D.H. Barlow (Eds.), Chronic anxiety: Generalized Anxiety Disorder and mixed anxiety-depression (pp. 1-28). New York: Guilford.

Barlow, D.H., Rapee, R.M. \& Brown, T.A. (1992). Behavioral treatment of generalized anxiety disorder. Behavior Therapy, 23, 551-570.

Bassler, M. (2004) Ätiopathogenese und Psychotherapie von Angststörungen aus psychodynamischer Sicht. In W. Hiller, E. Leibing, F. Leichsenring \& S. Sulz (Hrsg.), Lehrbuch der Psychotherapie für die Ausbildung zur/zum Psychologischen Psychotherapeuten und für die fachärztliche Weiterbildung. Band 2: Psychoanalytische und tiefenpsychologisch fundierte Therapie (S. 137-153). München: CIP-Medien.

Baum, A. \& Fleming, I, (1993). Implications of psychological research on stress and technological accidents. American Journal of Psychology, 48, 665-672.

Beck, A.T. (1967). Depression: Clinical, experimental and theoretical aspects. New York: Harper \& Row.

Beck, J.S. (1999). Praxis der Kognitiven Therapie. Weinheim: PVU, Beltz. 
Beck, J.S., Emery, G. \& Greenberg, R.L. (1985). Anxiety disorders and phobias. New York: Basic Books.

Bellak, J., Hurvich, M. \& Gediman, H. (1973). Ego functions in schizophrenics, neurotics, and normals. New York: Wiley.

Beutler, L.E. (1995). Common Factors and Specific Effects. Clinical Psychology: Science and Practice, 2, 79-82.

Blagys, M.D. \& Hilsenroth, M.J. (2000). Distinctive Features of Short-Term PsychodynamicInterpersonal Psychotherapy: A Review of the Comparative Psychotherapy Process Literature. Clinical Psychology: Science and Practice, 7, 167- 188.

Blagys, M.D. \& Hilsenroth, M.J. (2002). Distinctive Features of Short-Term CognitiveBehavioral Psychotherapy: A Review of the Comparative Psychotherapy Process Literature. Clinical Psychology Review, 22, 671-706.

Blazer, D., Hughes, D. \& George, L.K. (1987). Stressful life events and the onset of a generalized anxiety syndrome. American Journal of Psychiatry, 144, 1178-1183.

Bögels, S., Wijts, P. \& Sallaerts, S. (2003). Analytic psychotherapy versus cognitivebehavioral therapy for social phobia. Paper presented at: European Congress for Cognitive and Behavioural Therapies; September 2003; Prag.

Bolland, J. M. (1985). The search for structure: An alternative to the forced Q-sort technique. Political Methodology, 11, 91-107.

Borkovec, T.D. (1994). The nature, function and origins of worry. In G.C.L. Davey \& F. Tallis (Eds.), Worrying: Perspective on theory, assessment and treatment. New York: Wiley.

Borkovec, T.D. \& Costello, E. (1993). Efficacy of applied relaxation and cognitive-behavioral therapy in the treatment of generalized anxiety disorder. Journal of Consulting and Clinical Psychology, 61, 611-619.

Borkovec, T.D., Hazlett-Stevens, H. \& Diaz, M.L. (1999). The role of positive beliefs about worry in generalized anxiety disorder and its treatment. Clinical Psychology and Psychotherapy, 6, 126-138.

Borkovec, T.D., Ray, W.J. \& Stöber, J. (1998). Worry: A cognitive phenomenon intimately linked to affective, physiological, and interpersonal behavioral processes. Cognitive Therapy and Research, 22, 561-567. 
Borkovec, T.D., Robinson, E., Pruzinsky, T. \& DePree, J.A. (1983). Preliminary exploration of worry: Some 39 characteristics and processes. Behavior Research and Therapy, 21, 9-16.

Borkovec, T.D. \& Whisman, M.A. (1996). Psychosocial treatment for generalized anxiety disorder. In M.R. Mavissakalian \& R.F. Prien (Eds.), Long-term treatments of Anxiety Disorders (pp. 171-199). Washington: American Psychiatric Press.

Bortz, J. \& Döring, N. (1993). Forschungsmethoden und Evaluation für Sozialwissenschaftler. Berlin: Springer.

Bortz, J. \& Döring, N. (1995). Forschungsmethoden und Evaluation für Sozialwissenschaftler (2. Aufl.). Berlin: Springer.

Brawman-Mintzer, O. \& Lydiard, R.B. (1997), Biological basis of generalized anxiety disorder. Journal of Clinical Psychiatry, 58 (suppl 3), 16-25.

Brom, D., Kleber, R.J. \& Defares, P.B. (1989). Brief psychotherapy for posttraumatic stress disorders. Journal of Consulting and Clinical Psychology, 57, 607-612.

Brown, S. R. (1995). "The California Way of Q-Methodology". Multiple recipients of list QMETHOD. Verfügbar über: http://www.cios.org/q-method.htm (23. Jan 95).

Brown, T.A., O'Leary, T.A. \& Barlow, D.H. (1993). Generalized anxiety disorder. In D.H. Barlow (Ed.), Clinical handbook of psychological disorders (pp. 137-188). New York: Guilford Press.

Brunink, S.A. \& Schroeder, H.E. (1979). Verbal Therapeutic Behavior of Expert Psychoanalytically Oriented, Gestalt, and Behavior Therapists. Journal of Consulting and Clinical Psychology, 47, 567-574.

Bundespsychotherapeutenkammer (2007). Verfügbar unter: http://www.bptk.de/psychotherapie/zahlen_fakten/90104.html\#4

Carter, R.M., Wittchen, H.-U., Pfister, H. \& Kessler, R.C. (2001). One-year prevalence of subthreshold and threshold DSM-IV generalized anxiety disorder in a nationally representative sample. Depression and Anxiety, 13, 78-88.

Caspar, F. \& Jacobi, F. (2005). Psychotherapieforschung. In W. Hiller, E. Leibing, F. Leichsenring \& S.K.D. Sulz (Hrsg.), Lehrbuch der Psychotherapie, Band 1: Wissenschaftliche Grundlagen der Psychotherapie (3. Aufl.) (S. 395-410). München: CIP-Medien. 
Castonguay, L.G., Goldfried, M.R., Wiser, S., Raue, P.J. \& Hayes, A.M. (1996). Predicting the Effect of Cognitive Therapy for Depression: A Study of Unique and Common Factors. Journal of Consulting and Clinical Psychology, 64, 497-504.

Chambless, D.L. \& Hollon, S.D. (1998). Defining empirically supported treatments. Journal of Consulting and Clinical Psychology, 66, 7-18.

Chambless, D.L. \& Ollendick, T.H. (2001). Empirically Supported Psychological Interventions: Controversies and Evidence. Annual Review of Psychology, 52, 685716.

Cottle, C. E. \& McKeown, B. (1980). The forced-free distinction in Q technique: A note on unused categories in the Q sort continuum. Operant Subjectivity, 3, 58-63.

Crits-Christoph, P., Connolly, M.B., Azarian, K., Crits-Christoph, K. \& Shapell, S. (1996). An open trial of brief supportive-expressive psychotherapy in the treatment of generalized anxiety disorder. Psychotherapy, 33, 418-430.

Crits-Christoph, P., Wolf-Palcio, D., Fichter, M. \& Rudnick, D. (1995). Brief supportiveexpressive psychodynamic therapy for generalized anxiety disorder. Psychotherapy, 33, 418-430.

Davidson, J.R.T. (2001). Pharmacotherapy of generalized anxiety disorder. Journal of Clinical Psychiatry, 62 (suppl. 11), 46-50.

Davies-Osterkamp, S., Heigl-Evers, A., Bosse-Steuernagel, G. \& Alberti, L. (1987). Zur Interventionstechnik in der psychoanalytisch-interaktionellen und tiefenpsychologisch fundierten Gruppentherapie - eine empirische Untersuchung. Gruppenpsychotherapie und Gruppendynamik, 23, 22-35.

DeRubeis, R. \& Feeley, M. (1990). Determinants of change in cognitive therapy for depression. Cognitive Therapy and Research, 14, 469-482.

Dilling, H., Mombour, W. \& Schmidt, M.H. (Hrsg.).(1993). Internationale Klassifikation psychischer Störungen- ICD-10 Kapitel V (F) Klinisch-diagnostische Leitlinien. Bern: Huber.

Dilling, H., Mombour, W., Schmidt, M.H. \& Schulte-Markwort (Hrsg.) (2004). Internationale Klassifikation psychischer Störungen- ICD-10 Kapitel V(F) Diagnostische Kriterien für Forschung und Praxis. (3. korrigierte Aufl.). Bern: Huber. Dobson, K.S. (Ed.) (2000). Handbook of Cognitive-Behavioral Therapies ( $2^{\text {nd }}$ ed.). New York: Guilford Publications. 
Durham, R.C., Allan, T. \& Hackett, C.A. (1997). On predicting improvement and relapse in general anxiety disorder following psychotherapy. British Journal of Clinical Psychology, 36, 101-119.

Durham, R.C., Murphy, T., Allan, T., Richard, K., Treliving, L.R. \& Fenton, G.W. (1994). Cognitive Therapy, Analytic Psychotherapy and Anxiety Management Training for Generalized Disorder. British Journal of Clinical Psychiatry, 165, 315-323.

Elkin, I. (1994). The NIMH treatment of depression collaborative research program: Where we began and where we are. In S.L. Garfield \& A.E. Bergin (Eds.), Handbook of psychotherapy and behaviour change ( $4^{\text {th }}$ ed.) (pp. 114-139). New York: John Wiley. Elkin, I., Shea, M.T., Watkins, J.T., Imber, S.D., Sotsky, S.M., Collins, J.F., Glass, D.R., Pilkonis, P.A., Leber, W.R., Docherty, J.P., Fiester, S.J. \& Parloff, M.B. (1989). National Institute of Mental Health Treatment of Depression Collaborative Research Program. General Effectiveness of Treatments. Archives of General Psychiatry, 46, 971-982.

Elliott, R. (1991). Five dimensions of therapy process. Psychotherapy Research, 1, 92-103.

Eysenck, H.J. (1952). The effects of psychotherapy: An evaluation. Journal of Consulting Psychology, 16, 319-324.

Faller, H. (2004). Wirkfaktoren der psychodynamischen Psychotherapie. In H. Lang (Hrsg.), Was ist Psychotherapie und wodurch wirkt sie? (S. 135-146).Würzburg: Königshausen \& Neumann.

Field, A. (2005). Discovering Statistics Using SPSS. (2 ${ }^{\text {nd }}$ ed.). London: Sage.

Fisher, P.L. \& Durham, R. C. (1999). Recovery rates in generalized anxiety disorder following psychological therapy: an analysis of clinical significant change in the STAI-T across outcome studies. Psychological Medicine, 29, 1425-1434.

Frank, J.D. (1961). Persuasion and healing ( $2^{\text {nd }}$ ed.). Baltimore: Johns Hopkins University Press.

Frank, J. D. (1973). Persuasion and healing ( $2^{\text {nd }}$ rev. ed.). Baltimore: Johns Hopkins University Press.

Frank, J.D. (1982). Therapeutic components shared by all psychotherapies. In J.H. Harvey \& M.M. Parks (Eds.), The master lectures series, vol. 1: Psychotherapy research and behaviour change (pp. 9-37). Washington, DC: American Psychological Association. Frank, J.D. (1992). Die Heiler: Wirkungsweisen psychotherapeutischer Beeinflussung. Vom Schamanismus bis zu den modernen Therapien (2.Aufl.). Stuttgart: Klett-Cotta. 
Freud, S. (1912). Ratschläge für den Arzt bei der psychoanalytischen Behandlung.

Gesammelte Werke Band 8. Frankfurt: Fischer (1975).

Freud, S. (1913). Zur Einleitung der Behandlung. Gesammelte Werke Bd. 8. Frankfurt: Fischer (1975).

Freud, S. (1914). Erinnern, Wiederholen und Durcharbeiten. Gesammelte Werke Bd. 10. Frankfurt: Fischer (1975).

Freud, S. (1923). Das Ich und das Es. Leipzig, Wien, Zürich: Internationaler Psychoanalytischer Verlag.

Freud, S. (1937). Die endliche und unendliche Analyse. Gesammelte Werke Bd. 16. Frankfurt: Fischer (1975).

Frieswyk, S.H., Allen, J.G., Colson, D.B., Coyne, L., Gabbard, G.O., Horwitz, L. \& Newsom, G. (1986). Therapeutic Alliance: Its Place as a Process and Outcome Variable in Dynamic Psychotherapy Research. Journal of Consulting and Clinical Psychology, 54, 32-38.

Garner, D.M., Rockert, W., Davis, R., Garner, M.V., Olmsted, M.P. \& Eagle, M. (1993). Comparison of cognitive-behavioral and supportive-expressive therapy for bulimia nervosa. American Journal of Psychiatry, 150, 37-46.

Giles, T.R. (1983a). Probable Superiority of Behavioral Interventions - I: Traditional Comparative Outcome. Journal of Behaviour Therapy and Experimental Psychiatry, 14, 29-32.

Giles, T.R. (1983b). Probable Superiority of Behavioral Interventions - II: Empirical Status Of The Equivalence Of Therapies Hypothesis. Journal of Behaviour Therapy and Experimental Psychiatry, 14, 189-196.

Giles, T.R. (1984). Probable Superiority of Behavioral Interventions -III: Some Obstacles To Acceptance Of Findings. Journal of Behaviour Therapy and Experimental Psychiatry, 15, 23-26.

Göttert, R. \& Asendorpf, J. (1989). Eine deutsche Version des California-Child-Q-Sort: Kurzform. Zeitschrift für Entwicklungspsychologie und Pädagogische Psychologie, 11(1), 70-82.

Goldfried, M.R. (1991). Research issues in psychotherapy integration. Journal of Psychotherapy Integration, 1, 5-25.

Goldfried, M.R., Castonguay, L.G., Hayes, A.M., Drozd, J.F. \& Shapiro, D.A. (1997). A comparative analysis of the therapeutic focus in cognitive-behavioral and 
psychodynamic-interpersonal sessions. Journal of Consulting and Clinical Psychology, 65, 740-748.

Goldfried, M., Raue, P.J. \& Castonguay, L.G. (1998). The therapeutic focus in significant sessions of master therapists: a comparison of cognitive-behavioral and psychodynamic-interpersonal interventions. Journal of Consulting and Clinical Psychology, 66, 803-810.

Goldfried, M.R. \& Wolfe, B.E. (1996). Psychotherapy Practice and Research. American Psychologist, 51, 1007-1016.

Grawe, K. (1976). Differentielle Psychotherapie I. Stuttgart, Bern: Huber.

Grawe, K. (1992). Diskussionsforum: Psychotherapieforschung zu Beginn der neunziger Jahre. Psychologische Rundschau, 43, 132-162.

Grawe, K. (1995). Grundriß einer Allgemeinen Psychotherapie. Psychotherapeut, 40, 130145 .

Grawe, K. (1998). Von der Diagnose zur Gruppentherapie. Eine fachliche Stellungnahme zu Tschuschke et al.: Psychotherapieforschung - wie man es nicht machen sollte. Zeitschrift für Psychotherapie, Psychosomatik und Medizinische Psychologie, 48, 445448 .

Grawe, K., Donati, R. \& Bernauer, F. (1994). Psychotherapie im Wandel. Göttingen: Hogrefe.

Greenberg, L.S. (1986). Change Process Research. Journal of Consulting and Clinical Psychology, 54, 4-9.

Greenson, R.R. (1967). The working alliance and the transference neuroses. Psychoanalysis Quarterly, 34, 155-181.

Greve, W. \& Wentura, D. (1991). Wissenschaftliche Beobachtung in der Psychologie : eine Einführung. München : Qintessenz-Verlag.

Grissom, R.J. (1996). The magical number .7+- .2: Meta-meta-analysis of the probability of superior outcome in comparisons involving therapy, placebo, and control. Journal of Consulting and Clinical Psychology, 64, 973-982.

Hahlweg, K. (1995). Zur Förderung und Verbreitung psychologischer Verfahren. Ein APABericht. Zeitschrift für Klinische Psychologie, 24, 275-284.

Hamilton, M. (1976). HAMA - Hamilton Anxiety Scale. In W. Guy (Ed.), Assessment Manual for Psychopharmacology. Rockville, Maryland: NIMH-ECDEU.

Hamilton, M. (1986). The Hamilton rating for depression. In N. Sartorius \& T.A. Ban (Eds.), Assessment of depression (pp. 278-296). Berlin: Springer. 
Hautzinger, M. (2002a). BDI - Beck Depression Inventar. In E. Brähler, J. Schumacher \& B. Strauß (Hrsg.), Diagnostische Verfahren in der Psychotherapie (S. 32-34). Göttingen: Hogrefe.

Hautzinger, M. (2002b). HAMA - Hamilton Angst Skala. In E. Brähler, J. Schumacher \& B. Strauß (Hrsg.), Diagnostische Verfahren in der Psychotherapie (S. 154-156). Göttingen: Hogrefe.

Hautzinger, M. (2002c). HAMD - Hamilton Depression Skala. In E. Brähler, J. Schumacher \& B. Strauß (Hrsg.), Diagnostische Verfahren in der Psychotherapie (S. 157-160). Göttingen: Hogrefe.

Hautzinger, M., Bailer, M., Worrall, H. \& Keller, F. (2000). Beck-Depressions-Inventar (BDI). Testhandbuch (3. Aufl.). Bern: Huber.

Hill, C.E. \& Lambert, M.J. (2004). Methodological Issues in Studying Psychotherapy Process and Outcomes. In M.L. Lambert, (Ed.), Bergin and Garfield's Handbook of Psychotherapy and Behavior Change (5 ${ }^{\text {th }}$ ed.) (pp 84-135). New York: Wiley \& Sons.

Hiller, W. (2004). www.klinische-psychologie-mainz.de/Abteilung/downloads/materialien/ gad.pdf

Hilsenroth, M.J., Blagys, M.D., Ackerman, S.J., Bonge, D.R., \& Blais, M.A. (2005). Measuring Psychodynamic-Interpersonal and Cognitive-Behavioral Techniques: Development of the Comparative Psychotherapy Process Scale. Psychotherapy: Theory, Research, Practice, Training, 42, 340-356.

Hodgson, R. \& Rachman, S. (1974). Desynchrony in measures of fear. Behaviour Research and Therapy, 12, 319-326.

Hoffmann, S.O. (2000). Psychodynamische Therapie und Psychodynamische Verfahren. Psychotherapeut, 45, 52-54.

Hogue, A, Liddle, H.A., Rowe, C., Turner, R.M., Dakof, G.A. \& LaPann, K. (1998). Treatment Adherence and Differentiation in Individual Versus Family Therapy for Adolescent Substance Abuse. Journal of Counseling Psychology, 45, 104-114.

Horowitz, L.M., Strauß, B. \& Kordy, H. (2000). Inventar zur Erfassung Interpersonaler Probleme (IIP-D). Handanweisung (2. Aufl.). Weinheim: Beltz Test Gesellschaft.

Horowitz, L.M., Strauß, B. \& Kordy, H. (2002). IIP-D - Inventar zur Erfassung Interpersonaler Probleme. In E. Brähler, J. Schumacher \& B. Strauß (Hrsg.), Diagnostische Verfahren in der Psychotherapie (S. 206-209). Göttingen: Hogrefe. 
Hoyer, J., Becker, E.S. \& Roth, W.T. (2001). Characteristics of worry in GAD patients, social phobics, and controls. Anxiety \& Depression, 13, 89-96.

Hoyer, J., Beesdo, K., Becker, E.S. \& Wittchen, H.-U. (2003). Epidemiologie und nosologischer Status der Generalisierten Angststörung. Zeitschrift für Klinische Psychologie und Psychotherapie, 32, 267-275.

Huf, A. (1992). Psychotherapeutische Wirkfaktoren. Weinheim: Beltz.

Imber, S.D., Pilkonis, P.A., Sotsky, S.M., Elkin, I., Watkins, J.T., Collins, J.F., Shea, T.M., Leber, W.R. \& Glass, D.R. (1990). Mode-Specific Effects Among Three Treatments for Depression. Journal of Consulting and Clinical Psychology, 58, 352-359.

Jacobson, E. (1938). Progressive relaxation. Chicago: University of Chicago Press.

Jacobson, E. (2002). Entspannung als Therapie. Progressive Relaxation in Theorie und Praxis (5. Aufl.). Stuttgart: Klett-Cotta.

Jakobsen, T., Rudolf, G., Brockmann, J., Eckert, J., Huber, D., Klug, G., Grande, T., Keller, W., Staats, H. \& Leichsenring, F. (2007). Ergebnisse analytischer Langzeitpsychotherapien bei spezifischen psychischen Störungen: Verbesserungen in der Symptomatik und in interpersonellen Beziehungen. Zeitschrift für

Psychosomatische Medizin und Psychotherapie 53, 87-110.

Jones, E.E. (1985). Manual for the Psychotherapy Process Q-set. Unpublished manuscript, University of California, Berkeley.

Jones, E.E. (2000). Therapeutic Action: A Guide to Psychoanalytic Therapy. Northvale, New Jersey, London: Jason Aronson.

Jones, E.E., Cumming, J.D. \& Horowitz, M.J. (1988). Another Look at the Nonspecific Hypothesis of Therapeutic Effectiveness. Journal of Consulting and Clinical Psychology, 56, 48-55.

Jones, E.E., Cumming, J.D \& Pulos, S.M. (1993). Tracing Clinical Themes across Phases of Treatment by a Q-Set. In N.E. Miller, L. Luborsky, J.P. Barber \& J.P. Docherty (Eds.), Psychodynamic Treatment Research. A Handbook for Clinical Practice (pp. 14 -36).

New York: Basic Books.

Jones, E.E., Ghannam, J., Nigg, J.T. \& Dyer, J.F.P. (1993). A Paradigm for Single-Case Research: The Time Series Study of a Long-Term Psychotherapy for Depression. Journal of Consulting and Clinical Psychology, 61, 381-394. 
Jones, E.E., Hall, S.A. \& Parke, L.A. (1991). The Process of Change: The Berkeley Psychotherapy Research Group. In L. Beutler \& M. Crago (Eds.), Psychotherapy Research (pp. 98-107). Washington, D.C: APA.

Jones, E.E., Parke, L.A. \& Pulos, S.M. (1992). How therapy is conducted in the private consulting room: A multivariate description of brief dynamic treatments. Psychotherapy Research, 2, 16-30.

Jones, E.E. \& Price, P.B. (1998). Interaction structure and change in psychoanalytic therapy. In R. Bornstein \& J. Masling (Eds.). Empirical studies of the therapeutic hour (pp. 2762). Washington DC: American Psychological Association.

Jones, E.E. \& Pulos, S.M. (1993). Comparing the process in psychodynamic and cognitivebehavioral therapies. Journal of Consulting and Clinical Psychology, 61, 306-316. Jones, E.E. \& Windholz, M. (1990). The psychoanalytic case study: Toward a method for systematic inquiry. Journal of the American Psychoanalytic Association, 38, 9851009.

Kanfer, F.H. (1977). The many faces of self-control, or behavior modification changes its focus. In R.B. Stuart (ed.), Behavioral self-management (pp. 1-48). New York: Brunner/Mazel.

Kanfer, F.H. \& Phillips, J.S. (1975). Lerntheoretische Grundlagen der Verhaltenstherapie. München: Kindler.

Karasu, T.B. (1986). The specificity versus nonspecificity dilemma: Toward identifying therapeutic change agents. American Journal of Psychiatry, 143, 687-695.

Kazdin, A.E. (1986). Comparative Outcome Studies of Psychotherapy: Methodological Issues and Strategies. Journal of Consulting and Clinical Psychology, 54, 95-105.

Kendall, P.C., Holmbeck, G. \& Verduin, T. (2004). Methodology, Design, and Evaluation in Psychotherapy Research. In M.J. Lambert (Ed.), Bergin and Garfield's Handbook of Psychotherapy and Behavior Change (5 ${ }^{\text {th }}$ ed.) (pp. 16-43). New York: Wiley \& Sons.

Kendler, K.S., Karkowski, L.M. \& Prescott, C.A. (1998). Stressful life-events and major depression: Risk period, longterm contextual threat and diagnostic specificity. Journal of Nervous and Mental Disease, 186, 661-669.

Kendler, K.S., Neale, M.C., Kessler, R.C., Heath, A.C. \& Eaves, L.J. (1992). Generalized anxiety disorder in women: a population based twin study. Archives of General Psychiatry, 49, 267-272. 
Kernberg, O.F. (1988). Schwere Persönlichkeitsstörungen. Theorie, Diagnose, Behandlungsstrategien. Stuttgart: Klett-Cotta.

Kerr, S., Goldfried, M.R., Hayes, A.M., Castonguay, L.G. \& Goldsamt, L.A. (1992). Interpersonal And Intrapersonal Focus In Cognitive-Behavioral And PsychodynamicInterpersonal Therapies: A Preliminary Analysis Of The Sheffield Project. Psychotherapy Research, 2, 266-276.

Kessler, R.C., Keller, M.B. \& Wittchen, H.-U. (2001). The epidemiology of generalized anxiety disorders. Psychiatric Clinics of North America, 24, 19-39.

Kohut, H. (1973). Narzißmus : eine Theorie der psychoanalytischen Behandlung narzißtischer Persönlichkeitsstörungen. Frankfurt: Suhrkamp.

Kriz, J. (1994). Grundkonzepte der Psychotherapie: Eine Einführung (4. Auflage). Weinheim: Beltz, Psychologie Verlags Union.

Kröner-Herwig, B. (2004). Die Wirksamkeit von Verhaltenstherapie bei psychischen Störungen von Erwachsenen sowie Kindern und Jugendlichen. Tübingen: DGVTVerlag.

Laireiter, A.-R. (2000). Was heilt in der Verhaltenstherapie? Wirkfaktoren der Psychotherapie und Persönlichkeitsentwicklung aus der Sicht der Verhaltenstherapie. In M. Hochgerner \& E. Wildberger (Hrsg.), Was heilt in der Psychotherapie? Überlegungen zur Wirksamkeitsforschung und Methodenspezifische Denkweisen.

Psychotherapeutische Theorie und Praxis Band 5 (S. 193-243). Wien: Facultas Universitätsverlag.

Lambert, M.J. \& Ogles, B.M. (2004). The Efficacy und Effectiveness of Psychotherapy. In M.J. Lambert (Ed.), Bergin and Garfield's Handbook of Psychotherapy and Behavior Change ( $5^{\text {th }}$ ed.) (pp. 139-193). New York: Wiley \& Sons.

Landman, J. \& Dawes, R. (1982). Psychotherapy outcome- Smith and Glass' conclusions stand up under scrutiny. American Psychologist, 37, 504-516.

Lang, P.J. (1965). Experimental studies of desensitization psychotherapy. In J. Wolpe, A. Salter \& L.J. Reyne (Eds.), The conditioning therapies. The challenge in psychotherapy (pp. 38-50). New York: Holt, Rinehart \& Winston.

Lang, P.J. (1993). The three-system approach to emotion. In N. Birbaumer \& A. Öhmann (Eds.), The structure of emotion (pp 18-30). Seattle: Hogrefe \& Huber.

Laswell, H.D. (1935). Verbal references and physiological changes during the psychoanalytic interview: A preliminary communication. Psychoanalytic Review, 22, 241-247. 
Laswell, H.D. (1936). Certain prognostic changes during trial (psychoanalytic) interviews. Psychoanalytic Review, 23, 241-247.

Laswell, H. D. (1937). Veränderungen an einer Versuchsperson während einer kurzen Folge von psychoanalytischen Interviews. Imago, 23, 375-380.

Laux, L., Glanzmann, P., Schaffner, P. \& Spielberger, C.D. (2002). STAI- State-TraitAngstinventar. In E. Brähler, J. Schumacher \& B. Strauß (Hrsg.), Diagnostische Verfahren in der Psychotherapie (S. 348-352). Göttingen: Hogrefe.

Leibing, E., Rabung, S. \& Leichsenring, F. (2005). Ist psychodynamische Kurztherapie eine wirksame Behandlungsform bei psychischen Störungen? Forum der Psychoanalyse, $21,371-379$.

Leibing, E., Winkelbach, C. \& Leichsenring, F. (2003). Die Generalisierte AngststörungDarstellung eines kognitiv-behavioralen Behandlungsmanuals. Verhaltenstherapie und psychosoziale Praxis, 35, 517-529.

Leichsenring, F. (1996). Zur Meta-Analyse von Grawe und Mitarbeitern.

Gruppenpsychotherapie und Gruppendynamik, 32, 205-234.

Leichsenring, F. (2005). Are psychodynamic and psychoanalytic therapies effective? A review of empirical data. International Journal of Psychoanalysis, 86, 1-26.

Leichsenring, F., Hiller, W., Weissberg, M. \& Leibing, E. (2006). Cognitive-Behavioral Therapy and Psychodynamic Psychotherapy: Techniques, Efficacy, and Indications. American Journal of Psychotherapy, 60, 233-259.

Leichsenring, F., Winkelbach, C. \& Leibing, E. (2002). Die Generalisierte AngststörungKrankheitsbild, Diagnostik und Therapie. Zeitschrift für Psychosomatische Medizin und Psychotherapie, 48, 235-255.

Leichsenring, F., Winkelbach, C. \& Leibing, E. (2005). Psychoanalytisch-orientierte Fokaltherapie der Generalisierten Angststörung. Ein Manual. Psychotherapeut, 50, 258-264.

Linden, M., Bär, T., Zubrägel, D., Ahrens, B. \& Schlattmann, P. (2002). Wirksamkeit der Kognitiven Verhaltenstherapie bei Generalisierten Angsterkrankungen - Ergebnisse der Berliner KVT-GAD-Studie. Verhaltenstherapie, 12, 173-181.

Linden, M., Zubrägel, D., Bär, T., Franke, U. \& Schlattmann, P. (2005). Efficacy of Cognitive Behaviour Therapy in generalized Anxiety Disorders. Psychotherapy and Psychosomatics, 74, 36-42. 
Lipsey, M.W. \& Wilson, D.B. (1993). The efficacy of psychological, educational, and behavioral treatment: Confirmation from meta-analysis. American Psychologist, 48, 1181-1209.

Luborsky, L. (1976). Helping alliances in psychotherapy: the groundwork for a study of their relationship to its outcome. In J.L. Claghorn (ed.), Successful psychotherapy (pp- 92116). New York: Brunner/Mazel.

Luborsky, L. (1995). Einführung in die analytische Psychotherapie. Ein Lehrbuch. Göttingen: Vandenhoeck \& Ruprecht.

Luborsky, L. \& DeRubeis, R.J. (1984). The use of psychotherapy treatment manuals: A small revolution in psychotherapy research style. Clinical Psychology Review, 4, 5-14.

Luborsky, L., Diguer, L., Seligman, D.A., Rosenthal, R., Krause, E.D., Johnson, S., Halperin, G., Bishop, M., Berman, J.S. \& Schweizer, E. (1999). The researcher's own therapy allegiances: A "wild card" in comparisons of treatment efficacy. Clinical Psychology: Science and Practice, 6, 95-132.

Luborsky, L., Singer, B. \& Luborsky, L. (1975). Comparative Studies of Psychotherapies: Is It True That "Everyone Has Won and All Must Have Prizes?” Archives of General Psychiatry, 32, 995-1008.

Maercker, A. (2003). Systematische Desensibilisierung. In J. Margraf (Hrsg.), Lehrbuch der Verhaltenstherapie. Band 1 (S. 405-412). Berlin: Springer.

Maier, W., Gansicke, M., Freyberger, H.J., Linz, M., Heun, R. \& Lecrubier, Y. (2000). Generalized anxiety disorder (ICD-10) in primary care from a cross-cultural perspective: A valid diagnostic entity? Acta Psychiatrica Scandinavica, 101, 29-36.

Malik, M.L., Beutler, L.E., Alimohamed, S., Gallagher-Thompson, D. \& Thompson, L. (2003). Are All Cognitive Therapies Alike? A Comparison of Cognitive and Noncognitive Therapy Process and Implications for the Application of Empirically Supported Treatments. Journal of Consulting and Clinical Psychology, 71, 150-158. Margraf, J. (1994). Mini-Dips: Kurz-Interview bei psychischen Störungen. Berlin: Springer. Margraf, J. (2000). Lehrbuch der Verhaltenstherapie (2. Aufl.) Band 1. Berlin: Springer. Margraf, J. (2002). Mini-DIPS. Diagnostisches Kurzinterview bei psychischen Störungen. In E. Brähler, J. Schumacher \& B. Strauß (Hrsg.), Diagnostische Verfahren in der Psychotherapie (S. 252-255). Göttingen: Hogrefe.

Margraf, J. \& Ehlers, A. (2003). Das Beck-Angst-Inventar. Bern: Huber. 
Martin, D.J., Garske, J.P. \& Davis, M.K. (2000). Relation of the therapeutic alliance with outcome and other variables: A meta-analytic review. Journal of Consulting and Clinical Psychology, 68, 438-450.

Matthews, A. (1990). Why worry? The cognitive function of anxiety. Behavior research and Therapy, 28, 455-468.

Mayman, M. (1978). Trauma, stimulus barrier, ego boundaries and self-preservation: Ego psychology. In S. Smith (ed.), The human mind revisited: Essays in honor of Karl A. Menninger. New York: International University Press.

Meibach, R.C., Mullane, J.F. \& Binstok, G. (1987). A placebo-controlled multicenter trial of propranolol and chlordiazepoxide in the treatment of anxiety. Current Therapeutic Research, 41, 65-76.

Mertens, W. (2000). Grundlagen psychoanalytischer Psychotherapie. In W. Senf \& M. Broda (Hrsg.), Praxis der Psychotherapie (S. 130-167). Stuttgart, New York: Thieme.

Mertens, W. (2005). Grundlagen psychoanalytischer Psychotherapie. In W. Senf \& M. Broda (Hrsg.), Praxis der Psychotherapie (S. 196-237). Stuttgart, New York: Thieme.

Meyer, A.E. (1990a). Wodurch wirkt Psychotherapie? In H. Lang (Hrsg.), Wirkfaktoren der Psychotherapie (S. 179-189). Berlin, Heidelberg, New York: Springer.

Meyer, A.E. (1990b). Kommunale Faktoren in der Psychotherapie als Erklärung für nicht grob unterschiedliche Ergebnisse - Ein Mythos mehr in der Psychotherapieforschung? Psychotherapie, Psychosomatik, Medizinische Psychologie, 40, 152-157.

Meyer, T.J., Miller, M.L., Metzger, R.L. \& Borkovec, T.D. (1990). Development and validation of the Penn State Worry Questionnaire. Behaviour Research and Therapy, 28, 487-495.

Milrod, B., Busch, F., Cooper, A. \& Shapiro, T. (1997). Manual of panic-focused psychodynamic psychotherapy. Washington: American Psychiatric Press.

Moncher, F.J. \& Prinz, R.J. (1991). Treatment fidelity in outcome studies. Clinical Psychology Review, 11, 287-266.

Müller, F. H. \& Kals, E. (2004). Die Q-Methode. Ein innovatives Verfahren zur Erhebung subjektiver Einstellungen und Meinungen. Forum Qualitative Sozialforschung / Forum: Qualitative Social Research [On-line Journal], 5(2), Art. 34. Verfügbar über: http://www.qualitative-research.net/fqs-texte/2-04/2-04muellerkals-d.htm [11.08.2006]. 
Noyes, R., Clarkson, C., Crowe, R.R., Yates, W.R. \& McChesney, C.M. (1987). A family study of generalized anxiety disorder. American Journal of Psychiatry, 144, 10191024.

Noyes, R., Holt, C.S. \& Woodman, C.L. (1996). Natural course of anxiety disorders. In M. R. Mavissakalian \& R.F. Prien, Long-term treatments of anxiety disorders (pp. 148). Washington, DC: American Psychiatric Association.

Noyes, R., Woodman, C, Garvey, M.J., Cook, B.L., Suelzer, M., Clancy, I. \& Anderson, D.J. (1992). Generalized anxiety disorder vs. panic disorder. Distinguishing characteristics and patterns of comorbidity. Journal of Nervous Disorders, 180, 369-379.

Nutt, D.J. (2001). Neurobiological mechanisms in general anxiety disorders. Journal of Clinical Psychiatry, 62 (suppl. 11), 22-27.

Öst, L.G. (1987). Applied relaxation: Description of a coping technique and review of controlled studies. Behaviour, Research and Therapy, 25, 397-409.

Öst, L.G. \& Breitholz, E. (2000). Applied relaxation vs. cognitive therapy in the treatment of generalized cognitive disorder. Behaviour, Research and Therapy, 38, 777-790.

Omer, H. \& Dar, R. (1992). Changing trends in three decades of psychotherapy research: The flight from theory into pragmatics. Journal of Consulting and Clinical Psychology, 60, 88-93.

Orlinsky, D.E. \& Howard, K.I. (1987). A generic model of psychotherapy. Journal of Integrative and Eclectic Psychotherapy, 6, 6-27.

Orlinsky, D.E., Roennestad, M.H. \& Willutzki, U. (2004). Fifty Years of Psychotherapy Process-Outcome Research: Continuity and Change. In M.L. Lambert (Ed.), Bergin and Garfield's Handbook of Psychotherapy and Behavior Change $\left(5^{\text {th }}\right.$ ed.) (pp. 307389). New York: Wiley \& Sons.

Pine, F. (1990). Die vier Psychologien der Psychoanalyse und ihre Bedeutung für die Praxis. Forum der Psychoanalyse, 6, 232-249.

Pole, N. \&, Jones, E.E. (1998). The talking cure revisited : Content analysis of a two-year psychodynamic psychotherapy. Psychotherapy Research, 8, 171-189.

Pulos, S. \& Jones, E.E. (1987). A study of the differential validity of the Psychotherapy Process Q-Sort. Paper presented at the annual meeting of the Western Psychological Association, San Diego, CA.

Rapee, R.M. (1991). Generalized anxiety disorder: A review of Clinical features and theoretical concepts. Clinical Psychology Review 11, 419-440. 
Regier, D.A., Narrow, W.E., Rae, D.S., Manderscheid, R.W., Locke, B.Z. \& Goodwin, F.K. (1993). The de facto US mental and Addictive Disorders Service System:

Epidemiologic catchment Area prospective 1-year prevalence rates of disorders in services. Archives of General Psychiatry, 50, 85-94.

Reimer, C. \& Rüger, U. (2003). Tiefenpsychologisch fundierte Psychotherapie. In C. Reimer \& U. Rüger (2003), Psychodynamische Psychotherapien. Lehrbuch der tiefenpsychologisch orientierten Psychotherapie (S. 25-60) (2. Aufl.). Berlin: Springer.

Reinecker, H. (2000). Therapieforschung. In J. Margraf (Hrsg.), Lehrbuch der Verhaltenstherapie. Band 1: Grundlagen - Diagnostik - Verfahren Rahmenbedingungen (2.Aufl.) (S. 49-67). Berlin: Springer.

Reinecker, H. (2005). Verhaltenstherapie. In W. Senf \& M. Broda, Praxis der Psychotherapie (S. 260-305). Stuttgart: Thieme.

Richter, R. (1998). Ansatzpunkt Therapeut-Patient-Beziehung: Psychoanalytisch orientierte Psychotherapie. In. U. Baumann \& M. Perrez (Hrsg.), Klinische Psychologie Psychotherapie (2. Aufl.) (S. 416-430). Bern: Hans Huber.

Roemer, L., Borkovec, T.D., Posa, S. \& Lyonfields, J.D. (1991). Generalized anxiety disorder in an analogue population: The role of past trauma. Paper presented at the $25^{\text {th }}$ Annual Convention of the Association for the Advancement of Behavior Therapy, New York/NY.

Rogers, C. R. (1942a). Counseling and psychotherapy. Boston: Houghton Mifflin.

Rogers, C. R. (1942b). The use of electrically recorded interviews in improving psychotherapeutic techniques. American Journal of Orthopsychiatry, 12, 429-434.

Rogers, M.P., Warshaw, M.G., Goisman, R.M., Goldenberg, I., Rodriguez-Villa, F., Mallya, G., Freeman, S.A. \& Keller, M.B. (1999). Comparing primary and secondary generalized anxiety disorder in a long-term naturalistic study of anxiety disorders. Depression and Anxiety, 10, 1-7.

Rosenzweig, S. (1936). Some implicit common factors in diverse methods of psychotherapy. American Journal of Orthopsychiatry, 6, 412-415.

Roston, D., Lee, K.A. \& Vaillant, G.E (2005). A Q-sort Approach to Identifying Defenses. In G.E. Vaillant (Ed.), Ego Mechanisms of Defense. A Guide for Clinicians and Researchers (pp. 217-233). Washington, DC, London, England: American Psychiatric Press. 
Rüger, U. \& Bell, K. (2004). Historische Entwicklung und aktueller Stand der RichtlinienPsychotherapie in Deutschland. Zeitschrift für Psychosomatische Medizin und Psychotherapie, 50, 127-152.

Rüger, U. \& Reimer, C. (2003). Gemeinsame Merkmale und Charakteristika psychodynamischer Psychotherapieverfahren. In C. Reimer \& U. Rüger (2003), Psychodynamische Psychotherapien. Lehrbuch der tiefenpsychologisch orientierten Psychotherapie (2. Aufl.) (S. 3-22). Berlin: Springer.

Ruhmland, M. \& Margraf, J. (2001). Effektivität psychologischer Therapien von generalisierter Angststörung und sozialer Phobie: Meta-Analysen auf Störungsebene. Verhaltenstherapie, 11, 27-40.

Sandahl, C., Herlitz, K., Ahlin, G. \& Rönnberg, S. (1998). Time-limited group psychotherapy for moderately alcohol dependent patients: A randomized controlled clinical trial. Psychotherapy Research, 8, 361-378.

Saß, H., Wittchen, H.-U. \& Zaudig, M. (Hrsg.) (2000). Diagnostisches und Statistisches Manual Psychischer Störungen DSM-IV (3., verb. Aufl.). Göttingen: Hogrefe.

Shadish, W.R., Matt, G.E., Navarro, A.M., Siegle, G., Crits-Christoph, P., Hazelrigg, M.D., Jorm, A.F., Lyons, L.C., Nietzel, M.T., Prout, H.T., Robinson, L., Smith, M.L., Svartberg, M. \& Weiss, B. (1997). Evidence that therapy works in clinically representative conditions. Journal of Consulting and Clinical Psychology, 65, 355365.

Shapiro, D.A., Barkham, M., Rees, A., Hardy, G., Reynolds, S. \& Startup, M. (1994). Effects of Treatment Duration and Severity of Depression on the Effectiveness of CognitiveBehavioral and Psychodynamic-Interpersonal Psychotherapy. Journal of Consulting and Clinical Psychology, 62, 522-534.

Shapiro, D.A. \& Shapiro, D. (1982). Meta-analysis of comparative therapy outcome studies: A replication and refinement. Psychological Bulletin, 92, 581-604.

Shoham-Salomon, V. (1991). Studying therapeutic modules precedes the integration of models. Journal of Psychotherapy Integration, 1, 35-41.

Shrout, P.E. \& Fleiss, J.L. (1979). Intraclass correlations: Uses in assessing rater reliability. Psychological Bulletin, 86, 420-428.

Silove, D., Parker, G. \& Manicavasagar, V. (1990). Perceptions of general and specific therapist behaviors. Journal of Nervous and Mental Diseases, 178, 292-299. 


\section{Literaturverzeichnis}

Skovholt, T.M. \& Roennestad, M.H. (1995). The evolving professional self. Stages and themes in therapist and counsellor development. Chichester: Wiley.

Sloane, R.B., Staples, F.R., Cristol, A.H., Yorkston, N.J. \& Whipple, K. (1975). Short-term analytically oriented psychotherapy versus behavior therapy. American Journal of Psychiatry, 132, 373-377.

Smith, M.L. \& Glass, G.V. (1977). Meta-Analysis of Psychotherapy Outcome Studies. American Psychologist, 32, 752-760.

Smith, M.L., Glass, G.V. \& Miller, T.I. (1980). The benefits of psychotherapy. Baltimore: Johns Hopkins University Press.

Snifeos, P.E. (1972). Short-term psychotherapy and emotional crisis. Cambridge, MA: Harvard University Press.

Stephenson, W. (1951). Correlating persons instead of tests. Character and Personality, 4, 17-24.

Stephenson, W. (1953). The study of behavior: Q-technique and its methodology. Chicago: University of Chicago Press.

Stiles, W.B., Shapiro, D.A. \& Elliott, R. (1986). Are all psychotherapies equivalent" American Psychologist, 41, 165-180.

Stiles, W.B., Shapiro, D.A \& Firth-Cozens, J.A. (1988). Verbal Response Mode Use in Contrasting Psychotherapies: A Within-Subjects Comparison. Journal of Consulting and Clinical Psychology, 56, 727-733.

Stöber, J. \& Bittencourt, J. (2002). PSWQ-PW - Penn State Worry Questionnaire - Past Week. In E. Brähler, J. Schumacher \& B. Strauß (Hrsg.), Diagnostische Verfahren in der Psychotherapie (S. 291-293). Göttingen: Hogrefe.

Streeck, U. (2000). Psychoanalytische Therapieverfahren. In W. Senf \& M. Broda (Hrsg.), Praxis der Psychotherapie (S. 168-184). Stuttgart, New York: Thieme.

Streeck, U. (2005). Psychodynamische Therapieverfahren. In W. Senf \& M. Broda (Hrsg.), Praxis der Psychotherapie (S. 238-258). Stuttgart, New York: Thieme.

Suinn, R.M. \& Richardson, F. (1971). Anxiety Management Training: A Nonspecific Behavior Therapy Program for Anxiety Control. Behavior Therapy, 2, 498-510.

Svartberg, M. \& Stiles, T.C. (1991). Comparative effects of short-term psychodynamic psychotherapy: a meta-analysis. Journal of Consulting and Clinical Psychology, 59, 704-714. 
Svartberg, M., Stiles, T. \& Seltzer, M.H. (2004). Randomized controlled trial of the effectiveness of short-term dynamic psychotherapy and cognitive therapy for Cluster C personality disorders. American Journal of Psychiatry, 161, 810-817.

Thurstone, L.L. \& Chave, E.J. (1929). The measurement of attitude. Chicago: University of Chicago Press.

Tschuschke, V., Bänninger-Huber, E., Faller, H., Fikentscher, E., Fischer, G., Frohburg, I., Hager, W., Schiffler, A., Lamprecht, F., Leichsenring, F., Leuzinger-Bohleber, M., Rudolph, G. \& Kächele, H. (1998). Psychotherapieforschung - wie man es nicht machen sollte. Zeitschrift für Psychotherapie, Psychosomatik und Medizinische Psychologie, 48, 430-444.

Tschuschke, V., Kächele, H. \& Hölzer, M. (1994). Gibt es unterschiedlich effektive Formen von Psychotherapie? Psychotherapeut, 39, 281-297.

Turowsky, J. \& Barlow, D.H. (1996). Kapitel 5: Generalisiertes Angstsyndrom. In J. Margraf (Hrsg.), Lehrbuch der Verhaltenstherapie, Band 2: Störungen, Glossar (S. 87-106). Heidelberg: Springer.

Üstün, T.B. \& Sartorius, N. (Eds.) (1995). Mental illness in general health care: An international study. Chichester, New York: John Wiley \& Son.

Wallerstein, R.S. (1986). Forty-two lives in treatment. New York: Guilford Press. Waltz, J, Addis, M.E., Koerner, K. \& Jacobson, N.S. (1993). Testing the Integrity of a Psychotherapy Protocol: Assessment of Adherence and Competence. Journal of Consulting and Clinical Psychology, 61, 620-630.

Wampold, B.E. (2001). The great psychotherapy debate: Models, methods, and findings. Mahwah, NJ: Erlbaum.

Wampold, B.E., Mondin, G.W., Moody, M., Stich, F., Benson, K. und Ahn, H. (1997). A Meta-Analysis of Outcome Studies Comparing Bona Fide Psychotherapies: Empirically, “All must have prizes”. Psychological Bulletin, 122, 203- 215.

Watzke, B. (2002). Vergleich therapeutischer Prozessvariablen in psychoanalytisch und verhaltenstherapeutisch begründeten stationären Gruppenpsychotherapien. Unveröffentlichte Dissertation: Universität Hamburg.

Weinberger, J. (1995). Common factors aren't so common: The common factors dilemma. Clinical Psychology: Science and Practice, 2, 45-69.

Wells, A. (1997). Cognitive therapy of anxiety disorders. A practice manual and conceptual guide. Chichester: Wiley. 
Wells, A. (1999). A metacognitive model and therapy for Generalized Anxiety Disorder. Clinical Psychology and Psychotherapy, 6, 86-95.

Wells, A. \& Carter, K. (1999). Preliminary tests of a cognitive model of Generalized Anxiety Disorder. Behaviour Research and Therapy, 37, 585-594.

Weltgesundheitsorganisation (WHO) (1993). Internationale Klassifikation psychischer Störungen. ICD-10. Bern: Huber.

Winkelbach, C. (2007). Psychodynamische Kurzzeittherapie und kognitive Verhaltenstherapie bei generalisierter Angststörung - eine randomisierte, kontrollierte und manualisierte Therapiestudie. Unveröffentlichte Dissertation, in Vorbereitung, Georg-AugustUniversität Göttingen.

Wirtz, M. \& Caspar, F. (2002). Beurteilerübereinstimmung und Beurteilerreliabilität: Methoden zur Bestimmung und Verbesserung der Zuverlässigkeit von Einschätzungen mittels Kategoriensystemen und Ratingskalen. Göttingen: Hogrefe.

Wiser, S. \& Goldfried, M.R. (1996). Verbal Interventions In Significant PsychodynamicInterpersonal And Cognitive-Behavioral Therapy Sessions. Psychotherapy Research, 6, 309-319.

Wittchen, H.-U. (2001). Bedarfsgerechte Versorgung psychischer Störungen. Abschätzungen aufgrund epidemiologischer, bevölkerungsbezogener Daten. Stellungnahme im Zusammenhang mit der Befragung von Fachgesellschaften durch den Sachverständigenrat für die Konzertierte Aktion im Gesundheitswesen. Verfügbar unter: http://www.svr-gesundheit.de/Gutachten/Gutacht01/befragung/idnummern/265.pdf.

Wittchen, H.-U. \& Hoyer, J. (2001). Generalized anxiety disorder: Nature and course. Journal of Clinical Psychiatry, 62 (suppl. 11), 15-19.

Wittchen, H.-U., Zaudig, M. \& Fydrich, T. (1997). SKID. Strukturiertes Klinisches Interview für DSM-IV. Achse I und II. Göttingen: Hogrefe.

Wittchen, H-U., Zaudig, M. \& Fydrich, T. (2002). SKID. Strukturiertes Klinisches Interview für DSM-IV. In E. Brähler, J. Schumacher \& B. Strauß (Hrsg.), Diagnostische Verfahren in der Psychotherapie (S. 413-418). Göttingen: Hogrefe.

Wittchen, H.-U., Zhao, S., Kessler, R.C. \& Eaton, W.W. (1994). DSM-III-R generalized anxiety disorder in the National Comorbidity Survey. Archives of General Psychiatry, $51,335-364$. 


\section{Literaturverzeichnis}

Wittmann, W.W. \& Matt, G.E. (1986). Meta-Analyse als Integration von Forschungsergebnissen. Psychologische Rundschau, 37, 20-40.

Wolfe, B.E. \& Goldfried, M.R. (1988). Research on Psychotherapy Integration: Recommendations and Conclusions From an NIMH Workshop. Journal of Consulting and Clinical Psychology, 56, 448-451.

Wolpe, J. (1958). Psychotherapy by reciprocal inhibition. Stanford, CA: Stanford University Press.

Woods, J.H., Katz, J.L. \& Winger, G. (1992). Benzodiazepines: Use, abuse, and consequences. Pharmacological Reviews, 44, 151-347.

Woody, G.E., Luborsky, L., McLellan, A.T. \& O’Brien, C.P. (1990). Corrections and revised analyses for psychotherapy and methadone maintenance patients. Archives of General Psychiatry, 47, 788-789.

Yeaton, W.H. \& Sechrest, L. (1981). Critical dimensions in the choice and maintenance of successful treatments: Strength, integrity, and effectiveness. Journal of Consulting and Clinical Psychology, 49, 156-167.

Yonkers, K.A., Massion, A., Warshaw, M. \& Keller, M.B. (1996). Phenomenology and course of generalized anxiety disorder. British Journal of Psychiatry, 168, 308-313.

Zubrägel, D., Bär, T. \& Linden, M. (2003). Generalisierte Angststörung. In E. Leibing, W. Hiller \& S. Sulz (Hrsg.), Lehrbuch der Psychotherapie für die Ausbildung zum Psychologischen Psychotherapeuten und für die ärztliche Weiterbildung. Band 3: Verhaltenstherapie (S. 261-271). München: CIP-Medien.

Zubrägel, D. \& Linden, M. (2000). Generalisierte Angststörung. In M. Linden und M. Hautzinger (Hrsg.), Verhaltenstherapiemanual. Berlin: Springer. 
Anhang

\section{Anhang}

Anhang A: Manual zum Psychotherapie-Prozess Q-Sort, Items und der zur Beurteilung verwendete Protokollbogen

Anhang B: Ergebnisse der statistischen Analysen 


\section{Anhang A: Manual zum Psychotherapie Prozess Q-Sort - Deutsche Ausgabe}

Enrico E. Jones, University of California, Berkeley

Übersetzung von Cornelia Albani, Gerd Blaser und Uwe Jacobs

Ziel und Zweck der im Psychotherapie Prozess Q-Sort enthaltenen Items ist es, eine grundlegende Sprache für die Beschreibung und Klassifizierung des Psychotherapieprozesses bereitzustellen. Der Methode liegt die Annahme zugrunde, dass Psychotherapie einen interpersonellen Prozess darstellt. Sie soll aber im Hinblick auf spezielle Therapietheorien neutral bleiben und daher die Abbildung eines weiten Spektrums therapeutischer Interaktionen erlauben. Die Verwendung einer standardisierten Sprache und Bewertungsprozedur soll eine systematische Einschätzung von Patient-TherapeutInteraktionen ermöglichen. Anstelle kleiner Ausschnitte von Patienten- oder Therapeutenäußerungen werden komplette Therapiesitzungen anhand des Q-Sorts bewertet. Dies soll ermöglichen, dass wichtige Vorgänge erfasst und gewonnene Eindrücke bewertet werden können. Das Instrument wurde mit dem Anliegen entwickelt, ein Inventar bedeutungsvoller Bestandteile des Therapieprozesses bereitzustellen, das in vergleichenden Untersuchungen angewendet werden kann und auch Prä-Post-Studien ermöglicht.

Das Vorgehen ist relativ einfach. Zunächst werden die Prozessdaten untersucht und eine Formulierung über das Material erstellt. Anschließend werden die 100 Karten verwendet. Ordnen Sie die Items in eine Reihe von neun Kategorien, wobei an einem Ende der Reihe die Karten sortiert werden, die Sie bezüglich des Materials als besonders charakteristisch einschätzen und am anderen Ende jene Karten, welche Sie für am wenigsten charakteristisch halten.

Am einfachsten sortiert man, indem man zunächst drei Stapel bildet - jene Karten, die man für charakteristisch hält auf der einen Seite, jene, die man für uncharakteristisch hält auf der anderen Seite, und jene Karten, die dazwischen fallen, in der Mitte. In dieser Phase der Auswertung muss noch nicht auf die Anzahl der Karten auf den drei Stapeln geachtet werden. Wenn alle drei Stapel geordnet sind, könne sie weiter aufgeteilt werden, nun jedoch in einer bestimmten Verteilung. Die Verteilung der Karten auf die jeweiligen Kategorien erfolgt entsprechend der nachfolgenden Tabelle: 
Anhang A

\begin{tabular}{|l|l|l|}
\hline Kategorie & Anzahl der Items & $\begin{array}{l}\text { Bezeichnung der } \\
\text { Kategorie }\end{array}$ \\
\hline 9 & 5 & $\begin{array}{l}\text { Extrem charakteristisch oder } \\
\text { zutreffend }\end{array}$ \\
\hline 8 & 8 & $\begin{array}{l}\text { ziemlich oder zutreffend } \\
\text { einigermaßen charakteristisch } \\
\text { oder zutreffend }\end{array}$ \\
\hline 7 & 12 & $\begin{array}{l}\text { ein wenig charakteristisch } \\
\text { oder zutreffend } \\
\text { relativ neutral oder unwichtig }\end{array}$ \\
\hline 6 & 16 & $\begin{array}{l}\text { ein wenig uncharakteristisch } \\
\text { oder nicht zutreffend }\end{array}$ \\
\hline 5 & 18 & $\begin{array}{l}\text { einigermaßen } \\
\text { uncharakteristisch oder nicht } \\
\text { zutreffend }\end{array}$ \\
\hline 3 & 16 & $\begin{array}{l}\text { ziemlich uncharakteristisch } \\
\text { oder nicht zutreffend }\end{array}$ \\
\hline 2 & 12 & $\begin{array}{l}\text { extrem uncharakteristisch } \\
\text { oder nicht zutreffend }\end{array}$ \\
\hline 1 & 8 &
\end{tabular}

Sie mögen sich von den Vorgaben durch die Q-Sort-Items und die Anleitung zur Zuordnung zu den Kategorien etwas eingeschränkt fühlen. Wie auch andere inhaltsanalytische Verfahren ist der Q-Sort darauf angelegt, komplexe Interaktionen im Sinne der Forschungsökonomie auf eine handhabbare Größe zu reduzieren. Keines dieser Instrumente kann sich den realen interaktiven Prozessen in einer Psychotherapie perfekt anpassen oder sie vollständig erfassen. Die Zuordnung einer festgelegten Anzahl von Karten zu jeder Kategorie ist jenen Methoden empirisch überlegen, in denen ein Kliniker eine beliebige Anzahl von Karten den jeweiligen Kategorien zuweisen kann. Die Q-Items sind Ergebnis vielfältiger Überlegung und psychometrischer Analysen. Es ist nicht möglich, die Charakteristika oder Inhalte einer spezifischen Therapie durch die extreme Platzierung einzelner Items zu erfassen. Es ist jedoch fast immer möglich, dies durch die Verbindung von zwei oder mehreren Items zu erreichen. Das Q-Sort soll eine Beschreibung der Dimensionen des psychotherapeutischen Prozesses anhand der passenden Zuordnung einzelner Items und der daraus resultierenden Verteilung mehrerer Items ermöglichen.

Der Q-Sort beinhaltet drei Typen von Items:

1. Items, die die Haltung, das Verhalten oder Erleben des Patienten beschreiben;

2. Items, die Handlungen und Haltungen des Therapeuten widerspiegeln und

3. Items, welche die Art der Interaktion der Dyade bzw. die Atmosphäre des Austauschs beschreiben. 
Die Definitionen, Beschreibungen und Beispiele, die zu jedem Item gehören, sollen dabei helfen, die Unterschiede im Verständnis der Items auf ein Minimum zu beschränken. Das Manual sollte genau studiert werden. Von den Beurteilern wird erwartet, dass sie die Position eines ,allgemeinen Anderen“ einnehmen, d.h. eines Beobachters, der die Interaktion zwischen Patient und Therapeut von außen betrachtet. Beim Zuordnen der Karten sollten Sie sich fragen: Ist diese Haltung, dieses Verhalten oder Erleben klar vorhanden (oder nicht vorhanden? Falls der Befund nicht überzeugend ist, stellen Sie sich folgende Frage: In welchem Ausmaß ist es vorhanden oder nicht vorhanden? Suchen Sie nach spezifischen Befunden. Versuchen Sie, so offen und objektiv wie möglich zu sein. Vermeiden Sie z.B. Urteile darüber, ob eine spezielle therapeutische Aktivität aus einer bestimmten theoretischen Position effektiv oder ineffektiv, wünschenswert oder unerwünscht ist. Seien Sie sich Ihrer möglichen Voreingenommenheiten bezüglich „,idealer“ therapeutischer Interaktionen bewusst. Vor allem versuchen Sie, sich nicht von Ihren persönlichen Reaktionen auf den Therapeuten oder Patienten beeinflussen zu lassen; vermeiden Sie zum Beispiel, Ihre Bewertung davon beeinflussen zu lassen, ob Sie diese Person gerne als Ihren Therapeuten hätten oder davon, wie Sie selbst als Therapeut reagiert hätten.

Beurteiler sind gelegentlich bei der Bewertung einer Stunde nicht sicher, ob ein bestimmtes Item in die eher neutrale (oder unwichtige) Kategorie gehört oder in eine der uncharakteristischen Kategorien. Ein Item sollte der neutralen Kategorie zugeordnet werden, wenn es tatsächlich unwichtig bezüglich der Interaktion ist. Eine extreme Platzierung des Items in Richtung uncharakteristisch bedeutet, dass das Fehlen eines bestimmten Verhaltens oder Erlebens bemerkenswert ist und daher in der Q-Sort Beschreibung der Stunde erfasst werden sollte. Mit anderen Worte, das fehlen eines Ereignisses, welches für die Beschreibung der Stunde wichtig erscheint, kann dadurch erfasst werden, dass es in der uncharakteristischen Kategorie platziert wird statt in der neutralen. Für viele Items sind diesbezüglich in der Definition detaillierte Anleitungen gegeben. Beurteiler können mitunter eine hinreichende Evidenz für eine solche Einschätzung (oder auch diesbezüglich anderer Arten der Itemzuordnung) vermissen. Es sei jedoch darauf hingewiesen, dass in umfassenden Studien bereits gezeigt werden konnte, dass mit Geduld und Sorgfalt hohe Inter-Rater-Reliabilitäten erreicht werden können.

Um den Text kürzer und leichter lesbar zu gestalten, wird bei den nachfolgenden Formulierungen der Items jeweils nur die maskuline Form verwendet. 
Item 1: Der Patient äußert negative Gefühle (z.B. Kritik, Feindseligkeit) in Bezug auf den Therapeuten (vs. bewundernde oder anerkennende Bemerkungen).

- Eher am charakteristischen Pol platzieren, wenn der Patient Gefühle von Kritik, Abneigung, Neid, Verachtung, Wut oder Feindschaft gegenüber dem Therapeuten äußert (z.B. der Patient wirft dem Therapeuten vor, die Therapie nicht genügend zu strukturieren).

- Eher am uncharakteristischen Pol platzieren, wenn der Patient positive oder freundliche Gefühle ggü. dem Therapeuten ausdrückt (z.B. der Patient macht dem Therapeuten Komplimente).

Item 2: Der Therapeut lenkt die Aufmerksamkeit auf das nonverbale Verhalten des Patienten, z.B. auf die Körperhaltung oder Gesten.

- Eher am charakteristischen Pol platzieren, wenn der Therapeut die Aufmerksamkeit auf das nonverbale Verhalten des Patienten, wie z.B. Mimik, Erröten, Lachen, Räuspern oder Körperbewegungen, lenkt (z.B. der Therapeut weist darauf hin, dass der Patient lächelt, obwohl er sagt, er sei wütend).

- Eher am uncharakteristischen Pol platzieren, wenn das nonverbale Verhalten des Patienten nicht oder nur wenig thematisiert wird.

\section{Item 3: Die Äußerungen des Therapeuten zielen darauf ab, dem Patienten das Erzählen} zu erleichtern.

- Eher am charakteristischen Pol platzieren, wenn die Äußerungen oder das Verhalten des Therapeuten darauf hinweisen, dass er dem Klienten zuhört und ihn dazu ermuntert fortzufahren (z.B. durch ,mm-hmm, ja, sicher, richtig etc.“).

- Eher am uncharakteristischen Pol platzieren, wenn der Therapeut sich nicht in dieser das Erzählen erleichternden Art verhält (das Item bezieht sich nicht auf Fragen und explorierende Kommentare).

- Bitte beachten: Die Beurteilung basiert auf der Absicht des Therapeuten, nicht auf der Wirkung, d.h. nicht darauf, ob es gelingt, das Erzählen zu erleichtern.

\section{Item 4: Die Therapieziele des Patienten werden besprochen.}

- Eher am charakteristischen Pol platzieren, wenn darüber gesprochen wird, was der Patient in der Therapie erreichen möchte. Diese Wünsche oder Ziele können sich auf persönliche oder innere Veränderungen (z.B. „Ich beginne diese Therapie, um über meine Depression hinweg zu kommen“") oder Veränderungen der Lebensumstände (,,ich bin gespannt, ob die Therapie dazu führen wird, dass ich heirate") beziehen.

- Eher am uncharakteristischen Pol platzieren, wenn weder Therapeut noch Patient auf mögliche Konsequenzen oder Folgen der Therapie Bezug nehmen.

\section{Item 5: Der Patient hat Schwierigkeiten die Bemerkungen des Therapeuten zu verstehen.}

- Eher am charakteristischen Pol platzieren, wenn der Patient durch die Kommentare des Therapeuten verwirrt scheint; das kann sowohl Abwehrcharakter haben als auch durch mangelnde Klarheit seitens des Therapeuten bedingt sein (z.B. sagt der Patient wiederholt „Was? Wie bitte“ oder zeigt auf andere Art und Weise, dass er nicht versteht, was der Therapeut meint) 
- Eher am uncharakteristischen Pol platzieren, wenn der Patient die Bemerkungen des Therapeuten problemlos versteht.

\section{Item 6: Der Therapeut ist einfühlsam, auf den Patienten eingestellt, empathisch.}

- Eher am charakteristischen Pol platzieren, wenn der Therapeut in der Lage dazu ist, die ,innere Welt“ des Patienten so zu erfassen, als ob es seine eigene wäre; wenn der Therapeut sensitiv für die Gefühle des Patienten ist und das Verstandene in einer Art und Weise kommunizieren kann, die dem Patienten angemessen erscheint (z.B. der Therapeut äußert etwas, das anzeigt, dass er verstanden hat, wie der Patient sich in einer bestimmten Situation fühlt).

- Eher am uncharakteristischen Pol platzieren, wenn der Therapeut kein einfühlendes Verständnis für die Gefühle oder die Erlebnisse des Patienten zu haben scheint.

Item 7: Der Patient ist ängstlich oder angespannt (vs. ruhig und entspannt).

- Eher am charakteristischen Pol platzieren, wenn der Patient Anspannung, Angst oder Unruhe deutlich zeigt; dies kann durch direkte Aussagen geschehen (z.B. „Heute fühle ich mich nervös und angespannt") oder indirekte (z.B. stammeln, stottern etc.) oder durch andere Verhaltensweisen zum Ausdruck kommen .

- Eher am uncharakteristischen Pol platzieren, wenn der Patient ruhig oder entspannt wirkt oder sich wohl zu fühlen scheint.

\section{Item 8: Patient ist besorgt oder beunruhigt wegen seiner Abhängigkeit vom} Therapeuten (vs. fühlt sich wohl mit der Abhängigkeit oder will Abhängigkeit).

- Eher am charakteristischen Pol platzieren, wenn der Patient durch die Abhängigkeit besorgt wirkt oder sich unwohl zu fühlen scheint (z.B. ein Bedürfnis, sich vom Therapeuten zurückzuziehen, zeigt oder auf irgendeine Art und Weise die Besorgnis, er könne von der Therapie abhängig werden).

- Eher am uncharakteristischen Pol platzieren, wenn sich der Patient in der Abhängigkeit wohl zu fühlen scheint; dies kann sich in Äußerungen von Hilflosigkeit zeigen oder der Patient fühlt sich wohl oder zufrieden in einer abhängigen Beziehung zum Therapeuten.

- Als neutral beurteilen, wenn der Patient eine relative Unabhängigkeit in der therapeutischen Beziehung erlebt.

\section{Item 9: Der Therapeut ist distanziert, unbeteiligt (vs. empfänglich und affektiv involviert).}

- Ehr am charakteristischen Pol platzieren, wenn die Haltung des Therapeuten dem Patienten gegenüber kühl, förmlich und unbeteiligt wirkt oder von emotionaler Zurückhaltung oder Rückzug gekennzeichnet ist.

- Eher am uncharakteristischen Pol platzieren, wenn der Therapeut emotional aufgeschlossen für den Patienten und affektiv involviert ist.

\section{Item 10: Patient strebt größere Nähe mit dem Therapeuten an.}

- Eher am charakteristischen Pol platzieren, wenn der Patient sich entweder wünscht oder versucht, die therapeutische Beziehung in eine stärker gleichberechtigte (stärker soziale) oder persönlichere und intime Beziehung zu verwandeln (z.B. der Patient sorgt sich um den Therapeuten oder er versucht, etwas über das Privatleben des Therapeuten zu erfahren). 
- Eher am uncharakteristischen Pol platzieren, wenn der Patient keine größere Nähe zum Therapeuten zu suchen scheint.

\section{Item 11: Sexuelle Gefühle und Erfahrungen werden besprochen.}

- Eher am charakteristischen Pol platzieren, wenn die Sexualität des Patienten besprochen wird; dies kann Gespräche über sexuelle Probleme oder über sexuelle Gefühle und Phantasien oder auch über aktuelle sexuelle Erfahrungen beinhalten (z.B. der Patient spricht darüber, öfter mit seinem Partner Sex haben zu wollen).

- Eher am uncharakteristischen Pol platzieren, wenn der Patient weder über sexuelle noch über erotische Inhalte spricht.

\section{Item 12: In der Stunde wird geschwiegen.}

- Eher am charakteristischen Pol platzieren, wenn es oft Schweigen oder bedeutsame Pausen innerhalb der Stunde oder einige ausgedehnte Perioden des Schweigens gibt.

- Eher am uncharakteristischen Pol platzieren, wenn nur wenig Schweigen auftritt.

- Bitte beachten: Kurze Sprechpausen sollten solange nicht als Schweigen bewertet werden, wenn sie selten sind und nicht länger als einige Sekunden dauern.

\section{Item 13: Der Patient ist lebhaft.}

- Eher am charakteristischen Pol platzieren, wenn der Patient lebendig oder expressiv wirkt (z.B. der Patient reagiert lebhaft auf eine Interpretation des Therapeuten).

- Eher am uncharakteristischen Pol platzieren, wenn der Patient gelangweilt, teilnahmslos, matt oder lustlos erscheint.

\section{Item 14: Der Patient fühlt sich vom Therapeuten nicht verstanden.}

- Eher am charakteristischen Pol platzieren, wenn der Patient Sorgen darüber äußert oder den Eindruck vermittelt, sich missverstanden zu fühlen oder annimmt, dass der Therapeut ihn nicht verstehen kann (z.B. wenn eine Witwe daran zweifelt, ob der Therapeut ihre Situation nachvollziehen kann, da er noch nie in ihrer Lage war).

- Eher am uncharakteristischen Pol platzieren, wenn der Patient irgendwie vermittelt, dass er sich vom Therapeut verstanden fühlt (z.B. antwortet der Patient

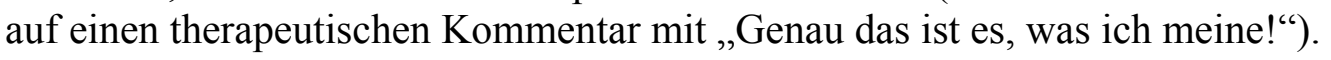

\section{Item 15: Patient initiiert keine Themen, ist passiv.}

- Eher am charakteristischen Pol platzieren, wenn der Patienten keine Gesprächsthemen oder Probleme einbringt oder keine Verantwortung für den Verlauf der Stunde übernehmen will (z.B. Patient sagt, dass er nicht weiß, worüber er sprechen soll).

- Eher am uncharakteristischen Pol platzieren, wenn der Patient das Schweigen oder Pausen durchbrechen will oder neue Themen spontan oder als Reaktion auf Nachfragen des Therapeuten einbringt und diese Themen dann aktiv (weiter)verfolgt oder vertieft.

Item 16: Es wird über Körperfunktionen, körperliche Symptome oder Gesundheit gesprochen. 
- Eher am charakteristischen Pol platzieren, wenn im Gespräch somatische Themen oder körperliche Symptome betont werden (z.B. der Patient berichtet von Müdigkeit oder Krankheit oder von Kopfschmerzen, Menstruationsschmerzen, wenig Appetit u.ä.).

- Eher am uncharakteristischen Pol platzieren, wenn körperliche Beschwerden kein wichtiges Gesprächsthema sind.

\section{Item 17: Der Therapeut kontrolliert die Interaktion aktiv (z.B. strukturiert und/oder führt neue Themen ein).}

- Eher am charakteristischen Pol platzieren, wenn der Therapeut häufig interveniert. Man sollte dieses Item aber nicht aufgrund der Angemessenheit der Interventionen beurteilen, sondern z.B. dann als charakteristisch beurteilen, wenn der Therapeut so aktiv ist, dass er häufig unterbricht oder interveniert, um Fragen zu stellen, etwas hervorzuheben, eine Meinung zu äußern oder er stark die Richtung innerhalb der Stunde vorgibt.

- Eher am uncharakteristischen Pol platzieren, wenn der Therapeut relativ selten interveniert und sich wenig anstrengt, die Interaktion zu strukturieren oder wenn der Therapeut der Führung des Patienten einfach nur folgt (z.B. Therapeut gestattet dem Patienten, wesentliche Themen einzubringen und hilft ihm dann dabei, einfach seinen Gedankengängen zu folgen).

\section{Item 18: Der Therapeut vermittelt Wertschätzung.}

- Eher am charakteristischen Pol platzieren, wenn der Therapeut den Patienten nicht offenen oder subtil negativ beurteilt; ,,inakzeptables“ oder problematisches Verhalten des Patienten wird so exploriert, dass dem Patienten das Gefühl vermittelt wird, wertgeschätzt zu sein.

- Ehr am uncharakteristischen Pol platzieren, wenn die Bemerkungen des Therapeuten oder sein Ton Kritik oder einen Mangel an Akzeptanz zum Ausdruck bringen. Eine Platzierung an das extremere Ende zeigt an, dass der Therapeut zum Ausdruck bringt, dass er die Charakterzüge oder die Persönlichkeit des Patienten als unangenehm empfindet.

\section{Item 19: Die therapeutische Beziehung hat eine erotische Färbung.}

- Eher am charakteristischen Pol platzieren, wenn die therapeutische Beziehung sexualisiert erscheint, angefangen von einer warmen, erotisch gefärbten Beziehung über schüchternem, verführerischem Verhalten des Patienten bis hin zu offen geäußerten Wünschen nach sexueller Befriedigung (z.B. der Patient spricht über sexuelle Erfahrungen als ob er die sexuellen Interessen des Therapeuten wecken wollte).

- Eher am uncharakteristischen Pol platzieren, wenn die therapeutische Beziehung im wesentlichen nicht sexualisiert erscheint; eine Bewertung hin zum uncharakteristischen Extrem zeigt an, dass der Therapeut oder der Patient Themen oder Verhaltensweisen, die sexuelles Interesse andeuten, meiden oder dass sexuelle Gefühle in den Griff bekommen oder unterdrückt werden.

\section{Item 20: Der Patient provoziert, testet die Grenzen der therapeutischen Beziehung. (Platzierung in der uncharakteristischen Richtung impliziert, dass der Patient sich angepasst verhält.)}

- Eher am charakteristischen Pol platzieren, wenn der Patient sich verhält, als ob er das Ziel hätte, eine emotionale Reaktion beim Therapeuten zu provozieren (z.B. 
der Patient versucht Zurückweisung durch den Therapeuten auszulösen, indem er Verhaltensweisen an den Tag legt, die den Therapeuten ärgern oder Inhalte der therapeutischen Vereinbarung verletzt).

- Eher am uncharakteristischen Pol platzieren, wenn der Patient besonders angepasst oder fügsam ist oder die Rolle des „guten Patienten“ zu spielen scheint, um den Therapeuten so für sich zu gewinnen.

\section{Item 21: Therapeutische Selbstöffnung.}

- Eher am charakteristischen Pol platzieren, wenn der Therapeut dem Patienten persönliche Informationen oder Reaktionen offenbart (z.B. der Therapeut erzählt dem Patienten, wo er aufgewachsen ist oder sagt zu ihm „Ich finde, Sie sind eine sehr liebenswürdige Person").

- Eher am uncharakteristischen Pol platzieren, wenn der Therapeut Abstand hält von therapeutischer Selbstöffnung. Eine Platzierung hin zum extremeren Ende bedeutet, dass der Therapeut sich selbst dann nicht öffnet, wenn der Patient ihn dazu drängt (z.B. Therapeut antwortet nicht direkt auf die Frage, ob der Therapeut verheiratet ist).

\section{Item 22: Der Therapeut fokussiert die Schuldgefühle des Patienten.}

- Eher am charakteristischen Pol platzieren, wenn der Therapeut die Schuldgefühle des Patienten fokussiert oder sonst irgendwie die Aufmerksamkeit darauf lenkt, v.a. dann, wenn die Absicht erkennbar ist, solche Gefühle mildern zu wollen (z.B. der Therapeut merkt an, dass die Patientin sich offensichtlich schuldig fühlte, als sie einmal nicht auf die andauernden Bitten um Hilfe von ihrer Tochter reagierte).

- Eher am uncharakteristischen Pol platzieren, wenn der Therapeut die Schuldgefühle des Patienten nicht betont.

\section{Item 23: Das Gespräch hat einen spezifischen Fokus.}

- Eher am charakteristischen Pol platzieren, wenn sich die Interaktion auf ein bestimmtes oder auf einige wenige Themen konzentriert (z.B. das beherrschende Thema der Stunde ist das Gefühl der Patientin, dass sie es zeitlebens und in vielerlei Hinsicht nicht geschafft hat, den Erwartungen ihres Vaters gerecht zu werden).

- Eher am uncharakteristischen Pol platzieren, wenn zahlreiche Themen besprochen werden oder wenn das Gespräch irgendwie diffus wirkt.

\section{Item 24: Die emotionalen Konflikte des Therapeuten stören die Beziehung.}

- Eher am charakteristischen Pol platzieren, wenn die Reaktionen des Therapeuten auf den Patienten ineffektiv oder unangemessenen scheinen und wenn sich diese Reaktion nicht ausschließlich nur aus der therapeutischen Interaktion ergibt, sondern sich möglicherweise aus den psychischen Konflikten des Therapeuten ableitet (z.B. Gegenübertragung, z.B. der Therapeut scheint bestimmte Affekte, die der Patient ausdrückt oder gerne ausdrücken möchte, zu meiden).

- Eher am uncharakteristischen Pol platzieren, wenn die persönlichen emotionalen Reaktionen des Therapeuten die Therapiebeziehung nicht unangemessen belasten.

\section{Item 25: Der Patient hat Schwierigkeiten die Stunde zu beginnen.}


- Eher am charakteristischen Pol platzieren, wenn der Patient zu Beginn der Stunde Unbehagen oder Verlegenheit zeigt (z.B. nach einer längeren Pause sagt der Patient „Ich weiß gar nicht, worüber ich heute sprechen soll“).

- Eher am uncharakteristischen Pol platzieren, wenn der Patient die Stunde direkt beginnt ohne längere Pausen oder Fragen des Therapeuten.

Item 26: Patient empfindet unangenehme oder schmerzliche Gefühle.

- Eher am charakteristischen Pol platzieren, wenn der Patient Scham, Schuld, Furcht oder Traurigkeit im Verlauf der Stunde ausdrückt. Eine extreme Bewertung bedeutet einen besonders intensiven Affekt.

- Eher am uncharakteristischen Pol platzieren, wenn der Patient keine solche unangenehmen Gefühle ausdrückt.

\section{Item 27: Therapeut gibt explizit Ratschläge und Anleitungen (vs. weigert sich, dies zu tun, selbst wenn er dazu gedrängt wird).}

- Eher am charakteristischen Pol platzieren, wenn der Therapeut explizit Ratschläge gibt oder bestimmte Vorschläge macht, die der Patient dann annehmen oder ablehnen kann (z.B. der Therapeut sagt „Wissen Sie, vielleicht sollten Sie einen Anwalt fragen, der Ihnen sagt, wie Sie ihre Erbschaft händeln sollen“) oder der Therapeut hält den Patienten dazu an, verschiedene Optionen zu bedenken und jede Alternative abzuwägen (z.B. der Therapeut weist auf die Möglichkeiten, die der Patient übersieht, hin und hält den Patienten dazu an, alle möglichen Konsequenzen seiner Handlungen abzuwägen).

- Eher am uncharakteristischen Pol platzieren, wenn der Therapeut keine Ratschläge gibt; eine extreme Platzierung in diese Richtung zeigt an, dass der Therapeut solche Ratschläge selbst dann nicht gibt, auch wenn der Patient danach verlangt oder es vielleicht hilfreich wäre.

\section{Item 28: Der Therapeut nimmt den therapeutischen Prozess richtig wahr.}

- Eher am charakteristischen Pol platzieren, wenn der Therapeut das Erleben des Patienten in der therapeutischen Beziehung richtig wahrnimmt. Dies soll aus den Kommentaren, Interventionen oder der generellen Haltung des Therapeuten dem Patienten gegenüber erschlossen werden. Die Beurteilung sollte unabhängig von der Art der Therapie (z.B. kognitiv-behavioral, psychoanalytisch) geschehen; sie soll aus der Perspektive der jeweiligen Therapieform vorgenommen werden.

- Eher am uncharakteristischen Pol platzieren, wenn der Therapeut den emotionalen Zustand des Patienten, die Bedeutung dessen, was er sagt, die Art der Interaktion zwischen ihnen nicht richtig wahrnimmt oder das Problem falsch formuliert.

\section{Item 29: Der Patient spricht davon, dass er Trennung von oder Distanz will.}

- Eher am charakteristischen Pol platzieren, wenn der Patient davon spricht, mehr Distanz oder eine Art Unabhängigkeit von jemandem (ausgenommen dem Therapeuten) haben zu wollen (z.B. äußert den Wunsch, endlich vom elterlichen Einfluss befreit sein zu wollen).

- Eher am uncharakteristischen Pol platzieren, wenn der Patient nicht darüber spricht, getrennt, unabhängig oder ungebunden sein zu wollen. 


\section{Item 30: Die Diskussion ist um kognitive Themen zentriert, d.h. Gedanken oder Überzeugungen.}

- Eher am charakteristischen Pol platzieren, wenn im Gespräch spezifische bewusste Vorstellungen, Themen, Überzeugungen, Glaubenssätze oder Konstrukte, betont werden, dazu dienen, andere, das Selbst oder die Welt zu beurteilen (z.B. der Therapeut schlägt vor, die Überzeugung des Patienten näher zu betrachten, wonach er wertlos sei, wenn nicht alles, was er in Angriff nimmt, perfekt gelingt.

- Eher am uncharakteristischen Pol platzieren, wenn nur wenig oder gar nicht von solchen Überzeugungen oder Konstrukten die Rede ist.

\section{Item 31: Der Therapeut fragt nach mehr Information oder ausführlicherer Schilderung.}

- Eher am charakteristischen Pol platzieren, wenn der Therapeut Fragen stellt, um mehr Informationen zu gewinnen oder den Patienten anhält, ein Erlebnis genauer zu beschreiben (z.B. der Therapeut fragt nach der Lebensgeschichte des Patienten oder danach, was dem Patienten durch den Kopf ging, als er zufällig einen Bekannten auf der Straße traf).

- Eher am uncharakteristischen Pol platzieren, wenn der Therapeut nicht aktiv Informationen sammelt.

Item 32: Der Patient gewinnt ein neues Verständnis oder Einsicht.

- Eher am charakteristischen Pol platzieren, wenn eine neue Perspektive, ein neuer Zusammenhang, eine neue Haltung oder abgewehrte Inhalte im Verlauf der Stunde auftauchen (z.B. im Anschluss an eine Bemerkung des Therapeuten wirkt der Patient nachdenklich und sagt „Ich glaube, das ist wahr. So habe ich das noch nie gesehen.

- Eher am uncharakteristischen Pol platzieren, wenn keine neue Einsicht im Verlauf der Stunde auftritt.

\section{Item 33: Der Patient spricht von Nähe oder vom Wunsch nach Nähe oder davon,} jemanden zu brauchen.

- Eher am charakteristischen Pol platzieren, wenn der Patient davon spricht, jemandem nah zu sein oder vom Wunsch nach Nähe und Intimität( jedoch nicht auf den Therapeuten bezogen) (z.B. der Patient sagt, dass er einsam ist und nicht allein sein möchte).

- Eher am uncharakteristischen Pol platzieren, wenn der Patient nicht über Nähe oder den Wunsch nach Nähe spricht.

\section{Item 34: Der Patient beschuldigt andere oder äußere Umstände, für (seine)} Schwierigkeiten verantwortlich zu sein.

- Eher am charakteristischen Pol platzieren, wenn der Patient dazu neigt zu externalisieren, andere oder äußere Umstände beschuldigt, für seine Schwierigkeiten verantwortlich zu sein (z.B. der Patient behauptet, dass seine/ihre Schwierigkeiten mit der Arbeit auf sein Pech mit Arbeitgebern zurückzuführen seien).

- Eher am uncharakteristischen Pol platzieren, wenn der Patient selbst Verantwortung für seine Schwierigkeiten übernimmt (z.B. feststellt, dass misslungene Beziehungen das Ergebnis seiner ungeeigneten Partnerwahl ist).

Item 35: Das Selbstbild ist ein Fokus des Gesprächs. 
- Eher am charakteristischen Pol platzieren, wenn, im positiven oder negativen, das Selbstkonzept oder Gefühle, Einstellungen oder Wahrnehmungen bezüglich der eigenen Person ein Gesprächsthema sind (z.B. die Patientin spricht darüber, dass es ihr manchmal schwer fällt, für sich selbst einzustehen, weil sie sich selbst dann als zu aggressiv erlebt).

- Eher am uncharakteristischen Pol platzieren, wenn das Selbstbild keine oder nur eine untergeordnete Rolle im Gespräch spielt.

\section{Item 36: Der Therapeut weist auf Abwehrmechanismen des Patienten hin, z.B. Ungeschehenmachen, Verleugnung.}

- Eher am charakteristischen Pol platzieren, wenn Abwehrmechanismen des Patienten, die darauf zielen, die Wahrnehmung bedrohlicher Informationen oder Gefühle zu verhindern, ein zentrales Thema darstellen (z.B. der Therapeut weist darauf hin, dass der Patient seine Liebe zu seinem Vater bezeugt, nachdem er gerade eine kritische Bemerkung über ihn gemacht hat).

- Eher am uncharakteristischen Pol platzieren, wenn diese Art von Abwehrdeutungen keine oder nur eine untergeordnete Rolle in der Sitzung spielt.

\section{Item 37: Der Therapeut verhält sich „lehrerhaft“.}

- Eher am charakteristischen Pol platzieren, wenn die Haltung des Therapeuten zum Patienten wie die eines Lehrers zu seinem Schüler ist. Dies sollte unabhängig vom konkreten Inhalt beurteilt werden, z.B. kann der Therapeut Informationen vermitteln oder Ratschläge geben oder Deutungen in Form von Anweisungen geben.

- Eher am uncharakteristischen Pol platzieren, wenn der Therapeut dem Patienten gegenüber keine Lehrer-Rolle einnimmt.

\section{Item 38: Es werden spezifische Aktivitäten oder Aufgaben besprochen, die der Patient außerhalb der Sitzungen in Angriff nehmen soll.}

- Eher am charakteristischen Pol platzieren, wenn bestimmte Aktivitäten besprochen werden, die der Patient außerhalb der Therapie in Angriff nehmen soll, wie z.B. die Gültigkeit einer spezifischen Überzeugung überprüfen, sich anders als gewöhnlich zu verhalten, Bücher lesen über seine Erkrankung etc. (z.B. Patient und Therapeut sprechen darüber, dass sich der Patient einer $/ \mathrm{m}$ angstauslösenden Situation oder Objekt stellen soll, die/das normalerweise gemieden wird).

- Eher am uncharakteristischen Pol platzieren, wenn nicht darüber gesprochen wird, solche Verhaltensweisen außerhalb der Therapie auszuprobieren.

\section{Item 39: Die Beziehung hat rivalisierenden Charakter.}

- Eher am charakteristischen Pol platzieren, wenn entweder der Patient oder der Therapeut mit dem jeweiligen anderen rivalisier. Dies kann in Form von Prahlen, sich aufwerten oder den anderen abwerten geschehen (z.B. der Patient deutet an, dass Therapeuten ein bequemes Leben führen während er selbst im richtigen Leben steht und hart arbeitet).

- Eher am uncharakteristischen Pol platzieren, wenn es nur wenig oder nicht rivalisieren. 
Item 40: Der Therapeut bezieht sich in seinen Interpretationen auf konkrete Personen im Leben des Patienten. (Eine Platzierung am uncharakteristischen Ende zeigt an, dass der Therapeut allgemeine, nicht auf konkrete Personen bezogene Interpretationen gibt).

- Eher am charakteristischen Pol platzieren, wenn sich die therapeutischen Interpretationen auf bestimmte Personen, die der Patient kennt, beziehen (z.B. sagt der Therapeut ,Sie fühlten sich verletzt und ärgerlich als Ihre Mutter Sie kritisierte").

- Eher am uncharakteristischen Pol platzieren, wenn die Interpretationen sich nicht auf bestimmte Personen oder auf andere Aspekte des Lebens des Patienten beziehen (z.B. sagt der Therapeut „Sie scheinen sich zurückzuziehen, wenn andere Ihnen nahe kommen").

Item 41: Die Ziele und Vorhaben des Patienten sind Gegenstand des Gesprächs.

- Eher am charakteristischen Pol platzieren, wenn der Patient über Vorhaben, Ziele oder Wünsche nach Erfolg und Status spricht (z.B. der Patient spricht über seine Hoffnungen, Anwalt zu werden und genügend Geld zu verdienen).

- Eher am uncharakteristischen Pol platzieren, wenn der Patient über eine Einengung in Bezug auf Zukunftserwartungen spricht, entweder in Form realistischer Planung oder von Wunschdenken.

\section{Item 42: Der Patient weist die Bemerkungen und Beobachtungen des Therapeuten zurück (vs. er nimmt sie an).}

- Eher am charakteristischen Pol platzieren, wenn der Patient mit den Vorschlägen, Beobachtungen oder Interpretationen des Therapeuten meist nicht übereinstimmt oder sie ignoriert (z.B. nach einer zentralen Interpretation des Therapeuten, merkt der Patient an, dass dies nicht den Punkt träfe).

- Eher am uncharakteristischen Pol platzieren, wenn der Patient dazu neigt, den therapeutischen Anmerkungen zuzustimmen.

\section{Item 43: Der Therapeut macht Vorschläge, wie das Verhalten anderer Personen zu} verstehen sei.

- Eher am charakteristischen Pol platzieren, wenn der Therapeut versucht, das Verhalten anderer Personen im Leben des Patienten zu interpretieren (z.B. der Therapeut vermutet, dass der Partner des Patienten Schwierigkeiten mit Intimität hat).

- Eher am uncharakteristischen Pol platzieren, wenn der Therapeut keine Äußerungen zur Bedeutung des Verhaltens anderer macht.

Item 44: Der Patient ist wachsam und misstrauisch (vs. vertrauensvoll und sicher).

- Eher am charakteristischen Pol platzieren, wenn der Patient wachsam, misstrauisch oder argwöhnisch dem Therapeuten gegenüber erscheint (z.B. der Patient fragt sich, ob der Therapeut ihn wirklich mag oder ob sich eine andere, versteckte Bedeutung in den Äußerungen des Therapeuten verbirgt.

- Eher am uncharakteristischen Pol platzieren, wenn der Patient vertrauensvoll, arglos und nicht misstrauisch ist.

Item 45: Der Therapeut nimmt eine supportive Haltung ein.

- Eher am charakteristischen Pol platzieren, wenn der Therapeut eine supportive, anwaltsartige Haltung dem Patienten gegenüber einnimmt. Dies kann in Form einer Bestätigung von Handlungen des Patienten sein oder als Ermutigung zu 
sozial kompetentem Verhalten. Oder der Therapeut stimmt positiven Selbstaussagen des Patienten zu oder betont die Stärken des Patienten (z.B. „Sie haben das schon in der Vergangenheit getan, also schaffen Sie es auch zukünftig“").

- Eher am uncharakteristischen Pol platzieren, wenn der Therapeut eher keine supportive Haltung in diesem Sinne einnimmt.

Item 46: Der Kommunikationsstil des Therapeuten ist klar und verständlich.

- Eher am charakteristischen Pol platzieren, wenn die Sprache des Therapeuten eindeutig, direkt und vollständig/leicht verständlich ist; Bewertung als sehr charakteristisch, wenn sein Sprachstil originell und außergewöhnlich ist.

- Eher am uncharakteristischen Pol platzieren, wenn die Sprache des Therapeuten diffus, eher abstrakt, stereotyp oder durch Jargon geprägt ist.

Item 47: Wenn die Beziehung mit dem Patienten schwierig ist, stellt sich der Therapeut darauf ein, um die Beziehung zu verbessern.

- Eher am charakteristischen Pol platzieren, wenn der Therapeut bereit und offen für Kompromisse und Anpassung scheint, wenn Missverständnisse oder Konflikte in der Dyade auftreten (z.B. wenn der Patient wegen des Therapeuten verärgert ist, versucht er oder sie ihn zu besänftigen).

- Eher am uncharakteristischen Pol platzieren, wenn der Therapeut keine Anstrengungen macht, die Beziehung zu verbessern, wenn Schwierigkeiten aufgetreten sind.

Item 48: Der Therapeut ermutigt den Patienten zur Unabhängigkeit im Handeln oder Denken.

- Eher am charakteristischen Pol platzieren, wenn der Therapeut den Patienten dazu ermuntert, selbständig zu denken und so zu handeln, wie er es selbst für am besten hält (z.B. der Therapeut merkt an, dass er nun von der Patientin gehört hat, was ihre Mutter und die Kollegen denken, was sie machen sollte, aber das noch nicht klar ist, was sie selbst davon hält und machen will).

- Eher am uncharakteristischen Pol platzieren, wenn der Therapeut das Thema Unabhängigkeit oder Initiative gar nicht aufgreift.

Item 49: Der Patient erlebt dem Therapeuten gegenüber ambivalente oder widersprüchliche Gefühle.

- Eher am charakteristischen Pol platzieren, wenn der Patient gemischte Gefühle über den Therapeuten äußert oder wenn der Inhalt der Äußerungen über den Therapeuten nicht im Einklang mit seinem Verhalten bzw. seinem üblichen Benehmen steht oder es eine Verdrängung von Gefühlen gibt (z.B. der Patient stimmt einem Vorschlag des Therapeuten überschwänglich zu, fährt dann jedoch mit Ausführungen fort, aus denen hervorgeht, dass er sich über Personen ärgert, die ihm vorschreiben, was er zu tun habe).

- Eher am uncharakteristischen Pol platzieren, wenn es nur schwache Anzeichen von Ambivalenz dem Therapeuten gegenüber gibt.

Item 50: Der Therapeut lenkt die Aufmerksamkeit auf Gefühle, die der Patient für inakzeptabel hält (z.B. Wut, Neid oder Aufregung). 
- Eher am charakteristischen Pol platzieren, wenn der Therapeut auf Gefühle hinweist oder diese betont, die der Patient für falsch, unangemessen oder gefährlich hält (z.B. der Therapeut merkt an, dass der Patient manchmal einen eifersüchtigen Hass auf seinen erfolgreicheren Bruder spürt).

- Eher am uncharakteristischen Pol platzieren, wenn der Therapeut nicht dazu neigt, emotionale Reaktionen zu betonen, die für den Patienten schwierig anzuerkennen oder zu akzeptieren sind.

\section{Item 51: Der Therapeut verhält sich herablassend oder behandelt den Patienten von oben herab.}

- Eher am charakteristischen Pol platzieren, wenn der Therapeut den Patienten von oben herab zu behandeln scheint als sei er weniger intelligent, gebildet oder intellektuell. Dies kann durch die Art, wie der Therapeut Ratschläge oder Kommentare gibt, geschlussfolgert werden.

- Eher am uncharakteristischen Pol platzieren, wenn der Therapeut mit seiner Art, seinem Ton oder seiner Stimme oder der Art seiner Kommentare vermittelt, dass er sich nicht für etwas Besseres hält.

\section{Item 52: Der Patient verlässt sich darauf, dass der Therapeut die Probleme des Patienten löst.}

- Eher am charakteristischen Pol platzieren, wenn der Patient seine Probleme in einer Art und Weise darzustellen scheint, die auf die Hoffnung oder Erwartung schließen lässt, dass der Therapeut spezifische Vorstellungen oder Ratschläge zu deren Lösung anbieten wird (z.B. der Patient äußert Unsicherheit darüber, ob er sich von seinem Partner trennen soll oder nicht und fragt den Therapeuten, was er machen soll). Dabei muss die Bitte nach einer Lösung nicht unbedingt explizit formuliert sein, sondern kann auch durch die Art, wie der Patient über das Problem spricht, deutlich werden.

- Eher am uncharakteristischen Pol platzieren, wenn der Patient sich weder explizit noch implizit darauf zu verlassen scheint, dass der Therapeut seine Probleme löst.

\section{Item 53: Der Patient ist damit beschäftigt, was der Therapeut von ihm denkt.}

- Ehr am charakteristischen Pol platzieren, wenn der Patient damit beschäftigt scheint, darüber nachzudenken, was der Therapeut von ihm oder seinem Verhalten denken könnte oder wie er von ihm bewertet wird (z.B. könnte der Patient anmerken „Sie denken bestimmt, dass das ganz dumm war"). Die Rater sollten dies auch aus dem Verhalten des Patienten schlussfolgern, z.B. wenn der Patient mit Fertigkeiten prahlt, um den Therapeuten zu beeindrucken.

- Eher am uncharakteristischen Pol platzieren, wenn der Patient nicht mit dem Eindruck, den er oder sie macht, beschäftigt scheint oder sich keine Sorgen bzgl. einer Bewertung durch den Therapeuten macht.

\section{Item 54: Der Patient ist in seiner Ausdrucksweise klar und strukturiert.}

- Eher am charakteristischen Pol platzieren, wenn der Patient sich in einer leicht verständlichen Art ausdrückt und relativ klar und fließend spricht.

- Eher am uncharakteristischen Pol platzieren, wenn die Sprache unzusammenhängend, vage oder häufig abschweifend ist. Dies kann sich manchmal darin äußern, dass es dem Beurteiler schwer fällt den roten Faden in den Themen, die der Patient anspricht, zu verfolgen. 
Item 55: Der Patient drückt positive Erwartungen bezüglich der Therapie aus.

- Eher am charakteristischen Pol platzieren, wenn der Patient die Hoffnung oder Erwartung ausdrückt, dass die Therapie ihm helfen wird. Eine Platzierung zum Extremen hin bedeutet, dass der Patient unrealistisch positive Erwartungen ausdrückt, z.B. dass die Therapie all ihre/seine Probleme lösen wird und ein Schutz gegen alle künftigen Schwierigkeiten sein wird (z.B. der Klient drückt die Hoffnung aus, dass die Therapie schnelle Ergebnisse erbringen wird).

- Eher am uncharakteristischen Pol platzieren, wenn der Patient die Therapie kritisiert, z.B. Enttäuschung darüber formuliert, dass die Therapie nicht effektiver oder befriedigender verläuft. Eine Einordnung zum Extremen hin bedeutet, dass der Patient skeptisch, pessimistisch oder desillusioniert darüber ist, was noch in der Therapie erreicht werden kann.

\section{Item 56: Der Patient spricht affektiv unbeteiligt über seine Erfahrungen. (Als neutral einschätzen, wenn Affekt und Bedeutung zwar offensichtlich sind, der Patient aber damit umgehen kann.)}

- Bezieht sich auf die Haltung, die der Patient zu dem, worüber gesprochen wird, einnimmt; wie ernst er es zu nehmen scheint, aber auch wie offen sein Affektausdruck ist.

- Eher am charakteristischen Pol platzieren, wenn der Patient wenig emotionale Beteiligung zeigt, generell eher flach, unpersönlich, hartherzig oder indifferent erscheint (Spannung/Erregung kann vorhanden sein oder nicht).

- Eine eher extreme Platzierung zum extrem uncharakteristischen Pol hin erfolgt, wenn der Patient intensive Gefühle ausdrückt, Gefühlsausbrüche hat und sich tief beunruhigt fühlt.

- Ehr als neutral einschätzen, wenn Affekt und Bedeutung zwar offensichtlich sind, der Patient diese aber kontrollieren kann und durch anderes, weniger affektives Material ausgleicht.

\section{Item 57: Der Therapeut erläutert das Rational seiner Technik oder seines Behandlungsansatzes.}

- Eher am charakteristischen Pol platzieren, wenn der Therapeut dem Patienten einige Aspekte der Therapie erläutert oder auf Fragen zum Behandlungsprozess antwortet (z.B. wenn der Therapeut auf eine direkte Frage oder Bitte des Patienten mit der Erläuterung antwortet, dass er es vorzieht, diese Frage nicht sofort zu beantworten, weil sich auf diese Weise die Gelegenheit bietet, den Gedanken und Gefühlen, die der Patient mit dieser Frage verbindet, besser nachzugehen). Mit diesem Item soll auch erfasst werden, wenn der Therapeut dem Patienten die Anwendung bestimmter therapeutischer Techniken vorschlägt oder ihn darin anleitet (z.B. schlägt der Therapeut vor, dass die Patientin versuchen soll, den Fokus auf ihre Gefühle zu verlagern, ihre Augen zu schließen und zu versuchen, sich eine bestimmte Szene vorzustellen oder in der Phantasie ein Gespräch mit jemandem während der Sitzung zu führen).

- Eher am uncharakteristischen Pol platzieren, wenn der Therapeut keine oder nur wenig Anstrengungen unternimmt, das Rational hinter einigen Aspekten der Behandlung zu erklären, selbst wenn er unter Druck steht oder es hilfreich sein könnte.

Item 58: Der Patient weigert sich, sich mit eigenen Gedanken, Reaktionen oder Motiven, die mit seinen Problemen in Zusammenhang stehen, auseinanderzusetzen. 
- Eher am charakteristischen Pol platzieren, wenn der Patient sich widersetzt, seine eigene Rolle bei der Aufrechterhaltung seiner Probleme zu untersuchen (z.B. der Patient umgeht, vermeidet oder verhindert bestimmte Themen oder wechselt wiederholt das Thema, wenn die Sprache auf dieses Thema kommt).

- Eher am uncharakteristischen Pol platzieren, wenn der Patient sich aktiv mit Gedankengängen auseinandersetzt, die emotional belastend oder erschütternd sind oder er in der Lage ist, diesen nachzugehen.

Item 59: Der Patient fühlt sich unzulänglich und unterlegen (vs. effektiv und überlegen).

- Eher am charakteristischen Pol platzieren, wenn der Patient Gefühle der Unzulänglichkeit, Unterlegenheit oder Unfähigkeit zum Ausdruck bringt (z.B. der Patient äußert, das sich nichts so entwickelt, wie er es sich wünscht).

- Eher am uncharakteristischen Pol platzieren, wenn der Patient Gefühle von Effektivität, Überlegenheit oder sogar Triumph ausdrückt (z.B. Patient zählt persönliche Erfolge auf oder beansprucht Beachtung für persönliche Eigenschaften oder Fertigkeiten).

Item 60: Der Patient hat eine kathartische Erfahrung. (Als uncharakteristisch einordnen, wenn einer emotionalen Äußerung keine Erleichterung folgt.)

- Eher am charakteristischen Pol platzieren, wenn der Patient Erleichterung erlebt, wenn er Zugang zu einem unterdrückten oder aufgestauten Gefühl gefunden hat (z.B. der Patient weint bitterlich über den Tod eines Elternteils und teilt dann dem Therapeuten mit, dass er sich besser fühlt dadurch, dass er seine Gefühle zugelassen hat).

- Eher am uncharakteristischen Pol platzieren, wenn auf das Erleben eines starken Affekts kein Gefühl der Entspannung oder der Erleichterung folgt.

- Als neutral einordnen, wenn die kathartische Erfahrung keine oder nur eine geringe Rolle in der Stunde spielt.

\section{Item 61: Der Patient fühlt sich schüchtern und verlegen (vs. selbstbewusst und selbstsicher).}

- Eher am charakteristischen Pol platzieren, wenn der Patient scheu, verlegen oder nicht selbstsicher erscheint. Zum Extremen hin bewerten, wenn er gedemütigt, gekränkt oder verletzt erscheint.

- Eher am uncharakteristischen Pol platzieren, wenn der Patient ungehemmt, zuversichtlich und selbstsicher erscheint.

\section{Item 62: Der Therapeut benennt ein sich in der Erfahrung oder dem Verhalten des Patienten wiederholendes Thema.}

- Eher am charakteristischen Pol platzieren, wenn der Therapeut ein wiederkehrendes Muster im Leben oder im Verhalten des Patienten aufzeigt (z.B. der Therapeut merkt an, dass der Patient sich Sexpartnern wiederholt auf einer bestimmten Schiene anbietet und so schäbiges Verhalten provoziert).

- Eher am uncharakteristischen Pol platzieren, wenn der Therapeut kein solches wiederkehrendes Muster oder Thema identifiziert.

\section{Item 63: Die zwischenmenschlichen Beziehungen des Patienten sind ein Hauptthema.}

- Eher am charakteristischen Pol platzieren, wenn der Hauptfokus des Gesprächs auf den sozialen oder beruflichen Beziehungen des Patienten oder bei den persönlichen emotionalen Belangen liegt (dies schließt die Diskussion über die 
therapeutische Beziehung (s. Item 98) und über Liebes- oder romantische Beziehungen (s. Item 64) aus) (z.B. der Patient berichtet, dass er unter den Konflikten mit seinem Chef leidet).

- Eher am uncharakteristischen Pol platzieren, wenn ein großer Teil der Stunde sich der Diskussion von Themen, die nicht direkt mit Beziehungen zusammenhängen, widmet (z.B. der Zwang des Patienten zu arbeiten, der Wunsch, etwas zu erreichen oder seine andauernde Beschäftigung mit Lebensmitteln und Essen).

- Bitte beachten: Dieses Item bezieht sich nicht auf Beziehungserfahrungen aus der Vergangenheit (s. Item 91 „Erinnerungen oder Rekonstruktionen der (frühen und späteren) Kindheit sind Gegenstand des Gesprächs“).

Item 64: Liebes- oder romantische Beziehungen werden besprochen.

- Eher am charakteristischen Pol platzieren, wenn romantische oder Liebesbeziehungen während der Sitzung besprochen werden (z.B. der Patient spricht über Gefühle für seinen Partner).

- Eher am uncharakteristischen Pol platzieren, wenn Liebesbeziehungen als Thema in der Stunde nicht auftauchen.

- Bitte beachten: Dieses Item bezieht sich auf die Qualität der Beziehung, nicht auf den Familienstand (z.B. ein Gespräch über die eheliche Beziehung, das nichts über Liebe oder Romantik aussagt, würde nicht als charakteristisch bewertet werden).

\section{Item 65: Der Therapeut klarifiziert, formuliert neu oder wiederholt mit anderen Worten, was der Patient sagte.}

- Eher am charakteristischen Pol platzieren, wenn ein Aspekt der Aktivität des Therapeuten darin besteht, dass er die affektive Stimmung, Äußerungen oder Ideen des Patienten neu formuliert oder umschreibt oder in eine etwas besser erkennbare Form bringt, um ihre Bedeutung zu verdeutlichen (z.B. sagt der Therapeut „Was Sie zu sagen scheinen, ist, dass Sie besorgt darüber sind, was in der Therapie passieren wird").

- Eher am uncharakteristischen Pol platzieren, wenn diese Art der Klarifikation selten vom Therapeuten während der Sitzungen benutzt wird.

Item 66: Der Therapeut beruhigt den Patienten. (Als uncharakteristisch einordnen, wenn der Therapeut dazu tendiert, den Patienten nicht explizit zu beruhigen.)

- Eher am charakteristischen Pol platzieren, wenn der Therapeut sich explizit bemüht, die Ängste des Patienten zu lindern und dem Patienten Mut zu machen, dass es besser wird (z.B. der Therapeut erzählt dem Patienten, dass es keinen Grund für Sorgen gibt und dass er sicher ist, dass sich sein Problem lösen lässt).

- Eher am uncharakteristischen Pol platzieren, wenn der Therapeut dazu tendiert, solch explizite Unterstützung auch dann nicht zu gewähren, wenn er dazu gedrängt wird.

Item 67: Der Therapeut interpretiert verdrängte oder unbewusste Wünsche, Gefühle oder Überzeugungen.

- Eher am charakteristischen Pol platzieren, wenn der Therapeut die Aufmerksamkeit des Patienten auf Gefühle, Gedanken oder Impulse lenkt, die ihm bisher nicht vollständig bewusst waren. Der Beurteiler muss versuchen, den Bewusstseinsgrad aus dem Kontext der Stunde abzuleiten (dies schließt aber die Interpretation von Abwehrmechanismen nicht mit ein, s. Item 36).

- Eher am uncharakteristischen Pol platzieren, wenn der Therapeut sich nur auf Material bezieht, dass dem Patienten völlig bewusst zu sein scheint. 
Item 68: Reale vs. phantasierte Bedeutungen von Erfahrungen werden unterschieden.

- Eher am charakteristischen Pol platzieren, wenn der Therapeut oder der Patient zwischen den Phantasien des Patienten über ein Ereignis und der objektiven Realität differenzieren (z.B. der Therapeut stellt heraus, dass, obwohl der Patient Todeswünsche für den Verstorbenen gehegt haben mag, er in der Realität dessen Herzanfall nicht verursacht hat). Verzerrte und fehlerhafte Annahmen sollten hier mit einbezogen werden (z.B. der Therapeut fragt, wie der Patient zu dieser Überzeugung gekommen sei, nachdem er die Welt wiederholt als gefährlich beschrieben hat).

- Eher am uncharakteristischen Pol platzieren, wenn sich innerhalb der Therapiestunde nur wenig mit verzerrten Wahrnehmungen der Realität beschäftigt wird.

\section{Item 69: Die aktuelle oder kurz zurückliegende Lebenssituation des Patienten wird} besprochen.

- Eher am charakteristischen Pol platzieren, wenn der Patient oder der Therapeut aktuelle oder kurz zurückliegende Lebensereignisse betonen (z.B. der Patient spricht über eine Depression aufgrund des kürzlichen Tods des Ehepartners).

- Eher am uncharakteristischen Pol platzieren, wenn Gespräche über die aktuelle Lebenssituation kein wichtiger Teil der Stunde waren.

Item 70: Der Patient ringt darum, Gefühle oder Impulse zu kontrollieren.

- Eher am charakteristischen Pol platzieren, wenn der Patient versucht, starke Emotionen oder Impulse zu managen, zu kontrollieren oder damit fertig zu werden (z.B. der Patient kämpft damit, Tränen zurückzuhalten, obwohl er offensichtlich sehr traurig ist).

- Eher am uncharakteristischen Pol platzieren, wenn der Patient keine Anstrengungen zu unternehmen scheint, seine Gefühle, die er erlebt, zurückzuhalten, zu kontrollieren oder zu lindern oder wenn er wenig oder keine Schwierigkeiten hat, sie zu kontrollieren.

\section{Item 71: Der Patient klagt sich selbst an, äußert Scham oder Schuld.}

- Eher am charakteristischen Pol platzieren, wenn der Patient Scham oder Schuld äußert oder sich selbst Vorwürfe macht (z.B. der Patient sagt, er hätte der schlechten Stimmung seiner Frau mehr Beachtung schenken sollen, dann hätte er ihren Selbstmord evtl. verhindern können).

- Eher am uncharakteristischen Pol platzieren, wenn der Patient nichts sagt, was auf Selbstanklage, Schamgefühle oder Gewissensbisse hinweist.

Item 72: Der Patient versteht das Wesen der Therapie und was von ihm erwartet wird.

- Eher am charakteristischen Pol platzieren, wenn der Patient zu verstehen scheint, was von ihm in der Situation erwartet und was in der Therapie passieren wird.

- Eher am uncharakteristischen Pol platzieren, wenn der Patient unsicher oder verwirrt ist oder seine Rolle in der Therapie und das, was von ihm in dieser Situation erwartet wird, missversteht.

\section{Item 73: Der Patient fühlt sich der therapeutischen Arbeit verpflichtet.}

- Eher am charakteristischen Pol platzieren, wenn der Patient sich der therapeutischen Arbeit verpflichtet zu fühlen scheint. Dies kann den Willen, Opfer 
(in Form von Zeit, Geld oder Unannehmlichkeiten) zu bringen, um die Therapie fortzusetzen, beinhalten ebenso wie den tiefen Wunsch, mehr über sich selbst zu erfahren, auch wenn dies psychische Belastung bedeuten könnte (z.B. dem Patienten war es so wichtig, die Therapie zu beginnen, dass er ein wöchentlich stattfindendes Golfspiel aufgibt, um zur Therapie zu kommen).

- Eher am uncharakteristischen Pol platzieren, wenn der Patient der Therapie gegenüber ambivalent zu sein scheint oder nicht willens ist, die emotionalen Anstrengungen und psychischen Belastungen, die sie bedeutet, in Kauf zu nehmen. Dies kann sich darin äußern, dass er sich über die Kosten der Therapie beschwert, es Terminprobleme gibt, er Zweifel über die Effektivität der Behandlung äußert oder er unsicher darüber ist, ob er Veränderungen überhaupt wünscht.

\section{Item 74: Es geht humorvoll zu.}

- Eher am charakteristischen Pol platzieren, wenn der Therapeut oder der Patient im Verlauf der Stunde Humor zeigen. Dies kann ein Abwehr- oder

Copingmechanismus beim Patienten sein; der Therapeut macht einen Witz oder ist ironisch, um etwas zu betonen oder um die Entwicklung des Arbeitsbündnisses zu stärken (z.B. die Patientin zeigt die Fähigkeit, über sich selbst oder ihre schwierige Lage zu lachen).

- Eher am uncharakteristischen Pol platzieren, wenn die Interaktion ernst, streng oder düster scheint.

\section{Item 75: Unterbrechungen in der Behandlung oder das Therapieende werden thematisiert.}

- Eher am charakteristischen Pol platzieren, wenn entweder der Patient oder der Therapeut über Unterbrechungen oder Pausen in der Behandlung sprechen (z.B. wegen Ferien oder Krankheit oder Beendigung der Therapie). Dies beinhaltet alle Äußerungen, die sich auf Unterbrechungen oder das Ende beziehen, egal ob dieses gewünscht wird, man sich davor fürchtet oder es droht.

- Eher am uncharakteristischen Pol platzieren, wenn versucht wird zu vermeiden, über Unterbrechungen oder Beendigung der Therapie zu sprechen (z.B. eine zu erwartende längere Pause in der Behandlung aufgrund der Sommerferien wird zwar erwähnt, aber weder Patient noch Therapeut gehen näher darauf ein).

- Als neutral zuordnen, wenn es gar kein Gespräch über Unterbrechungen der Behandlung oder über das Ende der Therapie gibt.

\section{Item 76: Der Therapeut legt dem Patienten nahe, die eigene Verantwortung für seine Probleme zu akzeptieren.}

- Eher am charakteristischen Pol platzieren, wenn der Therapeut dem Patienten nahe legt, dass er irgendwie handeln muss oder etwas verändern sollte, wenn sich seine Schwierigkeiten verbessern sollen (z.B. der Therapeut sagt: „Lassen Sie uns schauen, was sie möglicherweise dazu beigetragen haben, um diese Reaktion (einer anderen Person) hervorzurufen").

- Eher am uncharakteristischen Pol platzieren, wenn die Handlungen des Therapeuten nicht generell darauf abzielen, den Patienten die Verantwortung für seine Handlungen übernehmen zu lassen oder wenn der Therapeut dem Patienten nahe legt, dass er schon zu viel Verantwortung dafür übernimmt.

\section{Item 77: Der Therapeut ist taktlos.}


- Eher am charakteristischen Pol platzieren, wenn die Kommentare des Therapeuten so formuliert sind, dass der Patient sie sehr wahrscheinlich als verletzend oder herabsetzend empfindet. Diese Taktlosigkeit oder fehlende Sensibilität muss nicht auf einer Verärgerung oder Verwirrung des Therapeuten beruhen, sondern kann eher das Ergebnis eines Mangels an Technik, Umgangsformen oder verbalen Fähigkeiten sein.

- Eher am uncharakteristischen Pol platzieren, wenn die Kommentare des Therapeuten Freundlichkeit, Vorsicht oder Umsicht widerspiegeln.

\section{Item 78: Der Patient sucht die Zustimmung, Zuneigung oder Sympathie des Therapeuten.}

- Eher am charakteristischen Pol platzieren, wenn der Patient sich verhält, als ob er unbedingt vom Therapeuten gemocht werden möchte oder er unbedingt Aufmerksamkeit möchte oder er von ihm beruhigt werden will.

- Eher am uncharakteristischen Pol platzieren, wenn der Patient sich nicht so verhält.

\section{Item 79: Der Therapeut spricht eine Veränderung in der Stimmung oder dem Affekt des} Patienten an.

- Eher am charakteristischen Pol platzieren, wenn der Therapeut häufig oder deutlich Anmerkungen über Veränderungen in der Stimmung des Patienten oder der Qualität seines Erlebens macht (z.B. der Therapeut merkt an, dass der Patient als Reaktion auf seine Kommentare sein Verhalten von „Mach doch, was Du willst" hin zu einem Gefühl von Betroffenheit, aber einem ernsthafteren Arbeiten an seinen Problemen).

- Eher am uncharakteristischen Pol platzieren, wenn der Therapeut keine Äußerungen zu Veränderungen in der Stimmung des Patienten innerhalb der Stunde macht.

\section{Item 80: Der Therapeut bietet eine Erfahrung oder ein Ereignis aus einer anderen Perspektive an.}

- Eher am charakteristischen Pol platzieren, wenn der Therapeut eine Situation, die der Patient beschrieben hat, so wiedergibt, dass der Patient diese nun wahrscheinlich in einem anderen Licht sieht („Reframing“ oder ,kognitive Umstrukturierung"). Dem gleichen Inhalt wird so eine neue (und üblicherweise positivere) Bedeutung gegeben (z.B. nachdem der Patient sich selbst Vorwürfe gemacht hat, weil er einen schlimmen Streit mit seinem Partner begonnen hat, sagt der Therapeut „Vielleicht ist dies Ihre Art, auszudrücken, was Sie in dieser Beziehung brauchen"). Wird dieses Item bewertet, sollte ein bestimmtes Ereignis oder eine bestimmte Erfahrung reframed bzw. identifiziert werden.

- Eher am uncharakteristischen Pol platzieren, wenn dies nicht zu den wichtigen Aspekten während einer Therapiestunde gehört und der Therapeut dies nicht tut.

\section{Item 81: Der Therapeut betont die Gefühle des Patienten, um ihm zu helfen, diese intensiver zu erleben.}

- Eher am uncharakteristischen Pol platzieren, wenn der Therapeut den emotionalen Inhalt dessen, was der Patient beschreibt, hervorhebt, um ihm die emotionale Erfahrung dieses zu ermöglichen bzw. zu intensivieren (z.B. der Therapeut vermutet, dass die Interaktion, die der Patient gerade in Form einer Geschichte beiläufig erzählt hat, ihn möglicherweise sehr wütend gemacht hat). 
- Eher am uncharakteristischen Pol platzieren, wenn der Therapeut die Erfahrungen und Affekte des Patienten nicht betont oder eher an den objektiven Beschreibungen dieser interessiert scheint.

\section{Item 82: Der Therapeut benennt das Verhalten des Patienten in der Stunde auf eine Art und Weise, die bisher nicht explizit wahrgenommene Aspekte dieses Verhaltens deutlich werden lässt.}

- Eher am charakteristischen Pol platzieren, wenn der Therapeut regelmäßig oder einige sehr deutliche Kommentare über das Verhalten des Patienten während der Sitzung dergestalt macht, dass so ein neues Licht auf sie geworfen wird (z.B. der Therapeut nimmt an, dass das Zuspätkommen des Patienten zur Stunde eine Bedeutung hat oder der Therapeut merkt an, dass, wann immer er über emotionale Themen zu sprechen beginnt, er schnell zu einem anderen Thema wechselt).

- Eher am uncharakteristischen Pol platzieren, wenn der Therapeut das aktuelle Verhalten des Patienten in der Stunde nicht anspricht.

\section{Item 83: Der Patient ist fordernd.}

- Eher am charakteristischen Pol platzieren, wenn der Patient mehr als die üblichen Forderungen, Fragen und Bitten stellt oder den Therapeuten dazu bringen möchte, eine solche Forderung zu erfüllen (z.B. der Patient fordert Termine am Abend, medizinische Behandlung oder fordert mehr Struktur oder mehr Aktivität seitens des Therapeuten).

- Eher am uncharakteristischen Pol platzieren, wenn der Patient zögerlich oder zurückhaltend selbst bei normalen, angebrachten Forderungen an den Therapeuten ist (z.B. der Patient fragt nicht nach einem anderen Termin für die Sitzung, obwohl dieser Termin sich mit einem anderen, sehr wichtigen Termin überschneidet).

\section{Item 84: Der Patient drückt Ärger oder aggressive Gefühle aus.}

- Eher am charakteristischen Pol platzieren, wenn der Patient Groll, Wut, Verbitterung, Hass oder Aggression zum Ausdruck bringt (dies meint nicht solche Gefühle dem Therapeuten gegenüber, s. Item 1).

- Eher am uncharakteristischen Pol platzieren, wenn der Ausdruck solcher Gefühle nicht vorkommt oder der Patient Gefühle wie Liebe o.ä. ausdrückt.

\section{Item 85: Der Therapeut ermuntert den Patienten, neue Verhaltensweisen im Umgang} mit anderen auszuprobieren.

- Eher am charakteristischen Pol platzieren, wenn der Therapeut den Patienten dazu ermuntert, sich anders zu Personen zu verhalten als bisher (z.B. der Therapeut fragt den Patienten, was er glaubt, was passiert, wenn er seiner Mutter direkter sagt, wie es ihn trifft, wenn sie an ihm herumnörgelt). Eine Platzierung hin zum Extremen bedeutet, dass der Therapeut mit dem Patienten Beziehungsverhalten erarbeitet oder der Therapeut mit dem Patienten neue Verhaltensweisen anderen gegenüber einübt.

- Eher am uncharakteristischen Pol platzieren, wenn der Therapeut keine Vorschläge macht, wie der Patient mit anderen umgehen soll.

Item 86: Der Therapeut ist zuversichtlich oder selbstsicher (vs. unsicher oder defensiv).

- Eher am charakteristischen Pol platzieren, wenn der Therapeut zuversichtlich, sicher und nicht defensiv erscheint. 
- Eher am uncharakteristischen Pol platzieren, wenn der Therapeut unsicher oder verwirrt oder in Verlegenheit zu sein scheint.

\section{Item 87: Der Patient kontrolliert.}

- Eher am charakteristischen Pol platzieren, wenn der Patient einen Richtunggebenden, beherrschenden oder hindernden Einfluss auf die Stunde ausübt oder die Stunde lenkt (z.B. der Patient dominiert die Interaktion durch zwanghaftes Reden oder abruptes Unterbrechen des Therapeuten).

- Eher am uncharakteristischen Pol platzieren, wenn der Patient die Interaktion nicht kontrolliert, sondern mit dem Therapeuten gemeinsam arbeitet.

Item 88: Der Patient bringt relevante Themen und Material zur Sprache.

- Eher am charakteristischen Pol platzieren, wenn der Beurteiler einschätzt, dass die Dinge, die der Patient zur Sprache bringt und worüber er spricht, in Bezug auf die psychischen Konflikte wichtig sind oder dass diese Themen von großem Belang sind.

- Eher am uncharakteristischen Pol platzieren, wenn das Gespräch keinen richtigen Bezug zu wichtigen Themen zu haben scheint.

\section{Item 89: Der Therapeut stärkt die Abwehr.}

- Eher am charakteristischen Pol platzieren, wenn die Haltung des Therapeuten von einem ruhigen, aufmerksamen Entgegenkommen gekennzeichnet ist, mittels dem verhindert werden soll, dass der Patient emotional aus der Fassung gerät. Der Therapeut interveniert aktiv und hilft damit dem Patienten, störende Gedanken oder Gefühle zu unterdrücken oder zu vermeiden.

- Eher am uncharakteristischen Pol platzieren, wenn der Therapeut nichts tut, um das Abwehrverhalten zu stützen oder bedrohliche Gefühle und Gedanken zu unterdrücken.

\section{Item 90: Träume und Phantasien des Patienten werden besprochen.}

- Eher am charakteristischen Pol platzieren, wenn ein Bereich des Gesprächs sich auf Trauminhalte oder Phantasiematerial (Tag- oder Nachtträume) bezieht (z.B. Patient und Therapeut erörtern die möglichen Bedeutungen eines Traums des Patienten in der Nacht vor Beginn der Therapie).

- Eher am uncharakteristischen Pol platzieren, wenn es nur wenig oder gar kein Gespräch über Träume oder Phantasien während der Stunde gibt.

Item 91: Erinnerungen oder Rekonstruktionen der (frühen und späteren) Kindheit sind Gegenstand des Gesprächs.

- Eher am charakteristischen Pol platzieren, wenn ein Teil der Stunde dafür verwendet wird über die Kindheit oder frühe Lebensjahre zu sprechen.

- Eher am uncharakteristischen Pol platzieren, wenn wenig oder überhaupt nicht über diese Themen gesprochen wird.

\section{Item 92: Gefühle oder Wahrnehmungen des Patienten werden mit Situationen oder} Verhalten in der Vergangenheit verknüpft.

- Eher am charakteristischen Pol platzieren, wenn einige Verbindungen oder bemerkenswerte Zusammenhänge zwischen der aktuellen emotionalen Erfahrung des Patienten oder der Wahrnehmung der gegenwärtigen Ereignisse mit denen der Vergangenheit hergestellt werden (z.B. der Therapeut stellt heraus bzw. der Patient 
realisiert, dass aktuelle Verlustängste vom Verlust eines Elternteils während der Kindheit stammen).

- Eher am uncharakteristischen Pol platzieren, wenn aktuelle und vergangene Erfahrungen zwar besprochen, aber nicht miteinander verknüpft werden.

- Als neutral einordnen, wenn diese Themen wenig oder gar nicht besprochen werden.

\section{Item 93: Der Therapeut ist neutral.}

- Eher am charakteristischen Pol platzieren, wenn der Therapeut dazu tendiert, seine eigenen Meinungen oder Sichtweisen von Themen, die der Patient anspricht, zurückzuhalten. Der Therapeut scheint die Rolle eines neutralen Kommentators anzunehmen und die Sichtweise des Patienten auf diese Dinge wird im Gespräch hervorgehoben (z.B. die Therapeutin fragt, wie es für den Patienten wäre, wenn sie - als Therapeutin - den Ausdruck seiner Wut billigt und sich anschließend erkundigt wie es für ihn wäre, wenn sie diesen kritisieren würde).

- Eher am uncharakteristischen Pol platzieren, wenn der Therapeut Meinungen ausdrückt oder implizit oder explizit Position bezieht (z.B. der Therapeut sagt dem Patienten, dass es sehr wichtig ist, dass er lernt, wie er seine Wut ausdrücken kann oder, dass die Beziehung, in der dieser sich gerade befindet, nicht sehr gut ist und er lieber versuchen sollte, aus dieser herauszukommen).

- Bitte beachten: Neutralität ist nicht das gleiche wie Passivität. Der Therapeut kann aktiv sein und trotzdem eine neutrale Haltung einnehmen.

\section{Item 94: Der Patient fühlt sich traurig oder deprimiert (vs. fröhlich oder heiter).}

- Eher am charakteristischen Pol platzieren, wenn die Stimmung des Patienten melancholisch, traurig oder depressiv scheint.

- Eher am uncharakteristischen Pol platzieren, wenn der Patient entzückt oder froh scheint oder sonst irgendwie den Eindruck vermittelt, dass er sich wohl fühlt oder glücklich ist.

\section{Item 95: Der Patient fühlt sich unterstützt.}

- Eher am charakteristischen Pol platzieren, wenn der Patient irgendwie erleichtert oder ermutigt wirkt oder sich vom Therapeuten unterstützt fühlt.

- Eher am uncharakteristischen Pol platzieren, wenn der Patient sich über den Verlauf der Therapie enttäuscht fühlt, frustriert oder kontrolliert ist.

- Bitte beachten: Das Item bezieht sich nicht auf Ereignisse außerhalb der Therapie.

\section{Item 96: Terminvereinbarungen oder das Honorar werden besprochen.}

- Eher am charakteristischen Pol platzieren, wenn Patient und Therapeut über Terminvereinbarungen oder -Veränderungen (Zeit, Datum etc.) für die Therapiestunden oder über die Höhe des Honorars, den Zeitpunkt der Bezahlung u.ä. sprechen.

- Eher am uncharakteristischen Pol platzieren, wenn diese Themen nicht zur Sprache kommen.

Item 97: Der Patient ist introspektiv, er ist bereit, Gedanken und Gefühle zu erforschen.

- Eher am charakteristischen Pol platzieren, wenn der Patient offen und wenig blockiert wirkt. Er geht bei der Exploration seiner Gefühle und Gedanken über die üblichen Beschränkungen, Vorsicht, Bedenken und Empfindlichkeiten hinaus. 
- Eher am uncharakteristischen Pol platzieren, wenn das Gespräch zögerlich, zurückhaltend, reserviert, gezwungen oder gehemmt erscheint, der Patient steif ist und eben nicht ungezügelt, frei und unkontrolliert redet.

Item 98: Die therapeutische Beziehung ist ein Fokus des Gesprächs.

- Eher am charakteristischen Pol platzieren, wenn die therapeutische Beziehung besprochen wird (z.B. der Therapeut lenkt die Aufmerksamkeit auf Merkmale der Interaktion oder des interpersonalen Prozesses zwischen dem Patienten und sich selbst).

- Eher am uncharakteristischen Pol platzieren, wenn der Therapeut oder der Patient nicht über die Art der Interaktionen zwischen ihnen sprechen, sondern sich z.B. nur auf den Inhalt beschränken.

\section{Item 99: Der Therapeut fragt die Sichtweise des Patienten an (vs. bestätigt die Wahrnehmungen des Patienten).}

- Eher am charakteristischen Pol platzieren, wenn der Therapeut in irgendeiner Form die Sicht des Patienten bzgl. einer Erfahrung oder eines Ereignisses hinterfragt (z.B. der Therapeut könnte sagen „Wie kommt es, dass es so ist?“ oder „Das wundert mich bzw. ich frage mich, ob...?“ oder einfach „Oh?"). (Dieses Item bezieht sich nicht auf Interpretationen oder „reframing“ im Sinne davon, dass eine neue oder andere Bedeutung des vom Patienten Gesagten erzielt werden soll, sondern es bezieht sich statt dessen nur auf die Frage über die Sichtweisen des Patienten.)

- Eher am uncharakteristischen Pol platzieren, wenn der Therapeut mit der Perspektive des Patienten übereinstimmt, ihr nicht widerspricht oder ihre Richtigkeit bestätigt (z.B. der Therapeut sagt „Ich glaube, damit haben Sie recht“ oder „Sie scheinen sich hiermit gut auszukennen“).

\section{Item 100: Der Therapeut stellt Verbindungen zwischen der therapeutischen Beziehung und anderen Beziehungen des Patienten her.}

- Eher am charakteristischen Pol platzieren, wenn der Therapeut mehrere oder einige deutliche Aussagen trifft, die die Gefühle des Patienten für den Therapeuten und die Gefühle des Patienten zu anderen bedeutsamen Individuen in seinem Leben, miteinander verknüpfen. Dies kann sich auf aktuelle Beziehungen, vergangene oder aktuelle Beziehungen zu den Eltern (Verbindung zwischen der Übertragung und den Eltern) beziehen (z.B. die Therapeutin merkt an, dass sie denkt, dass die Patientin manchmal Angst davor hat, dass sie sie genauso kritisieren könnte wie ihre Mutter das immer tut).

- Eher am uncharakteristischen Pol platzieren, wenn die Handlungen des Therapeuten während der Stunde keine oder nur wenige Bemühungen beinhalten, die interpersonellen Aspekte der Therapie mit Erfahrungen in anderen Beziehungen zu verknüpfen. 
Anhang A: „Protokollbogen zur Beurteilung“

„Therapeutische Beziehung“

\begin{tabular}{|c|c|c|c|}
\hline \multicolumn{2}{|c|}{ Patient... } & \multicolumn{2}{|c|}{ Therapeut... } \\
\hline \multicolumn{2}{|c|}{ 1: ... äußert in Bezug auf den Therapeuten... } & \multicolumn{2}{|c|}{$\begin{array}{l}\text { 47: stellt sich auf die schwierige } \\
\text { Beziehung... }\end{array}$} \\
\hline $\begin{array}{l}\text {...negative } \\
\text { Gefühle/Kritik/Wut/Neid. }\end{array}$ & $\begin{array}{l}\text {..positive/freundliche } \\
\text { Gefühle/Komplimente. }\end{array}$ & \multirow[t]{3}{*}{$\begin{array}{l}\text {...ein und } \\
\text { versucht sie zu } \\
\text { verbessern. }\end{array}$} & \multirow[t]{3}{*}{$\begin{array}{l}\text {...nicht ein und } \\
\text { unternimmt nichts, } \\
\text { um sie zu } \\
\text { verbessern. }\end{array}$} \\
\hline \multicolumn{2}{|l|}{ 20: ... verhält sich } & & \\
\hline $\begin{array}{l}\text {...provozierend, will } \\
\text { emotionale Reaktion } \\
\text { beim T hervorrufen. }\end{array}$ & $\begin{array}{l}\text {... angepasst, fügsam, } \\
\text { spielt ,guter Patient““. }\end{array}$ & & \\
\hline \multicolumn{2}{|c|}{$\begin{array}{l}\text { 8: ... ist in Bezug auf die Abhängigkeit zum } \\
\text { Therapeuten... }\end{array}$} & & \\
\hline $\begin{array}{l}\text {.. besorgt, fühlt ich } \\
\text { unwohl damit. }\end{array}$ & $\begin{array}{l}\text {... fühlt sich wohl } \\
\text { damit. }\end{array}$ & & \\
\hline \multicolumn{2}{|c|}{ 10: strebt in Bezug zum Therapeuten... } & \multicolumn{2}{|c|}{ 21: therapeutische Selbstöffnung... } \\
\hline $\begin{array}{l}\text {...größere Nähe, mehr } \\
\text { Gleichberechtigung und } \\
\text { Intimität an. }\end{array}$ & $\begin{array}{l}\text {...keine größere Nähe } \\
\text { an. }\end{array}$ & $\begin{array}{l}\ldots \mathrm{T} \text { gibt } \\
\text { Infos/Reaktionen } \\
\text { von sich preis. }\end{array}$ & $\begin{array}{l}\text { T weigert sich, } \\
\text { solche Infos von sich } \\
\text { preis zu geben. }\end{array}$ \\
\hline \multicolumn{2}{|c|}{ 14: ... fühlt sich vom Therapeuten... } & \multicolumn{2}{|c|}{ 6: ... ist in Bezug zum Patienten... } \\
\hline \multirow[t]{4}{*}{$\begin{array}{l}\text {... nicht verstanden, } \\
\text { missverstanden. }\end{array}$} & $\begin{array}{l}\text {... gut oder richtig } \\
\text { verstanden. }\end{array}$ & $\begin{array}{l}\text {...empathisch, } \\
\text { kann die innere } \\
\text { Welt von ihm } \\
\text { erfassen. }\end{array}$ & $\begin{array}{l}\text {...unempathisch, } \\
\text { kann seine } \\
\text { Gefühle/Erfahrungen } \\
\text { nicht verstehen. }\end{array}$ \\
\hline & & \multicolumn{2}{|c|}{ 18: ... vermittelt Wertschätzung (+/-). } \\
\hline & & \multicolumn{2}{|c|}{$\begin{array}{l}\text { 28: ...nimmt den therapeutischen } \\
\text { Prozess... }\end{array}$} \\
\hline & & ...richtig wahr. & $\begin{array}{l}\text {... nicht richtig } \\
\text { wahr. }\end{array}$ \\
\hline \multicolumn{4}{|c|}{ 98: Therapeutische Beziehung $\rightarrow$ Fokus des Gesprächs? } \\
\hline 100: verbindet $T$ thera & & hungen? & \\
\hline
\end{tabular}




\section{„Umgang mit Gefühlen“}

Der Patient...

\begin{tabular}{|l|l|}
\hline 84. ... drückt Ärger, aggressive Gefühle aus. & \\
\hline 71. ... klagt sich an, äußert Scham / Schuld. & \\
\hline 60. ... hat eine kathartische Erfahrung. & \\
\hline
\end{tabular}

Der Therapeut...

\begin{tabular}{|l|l|}
\hline 81. ... betont Gefühle des P. & \\
\hline 22. ... fokussiert Schuldgefühle. & \\
\hline $\begin{array}{l}\text { 50. ... lenkt Aufmerksamkeit auf für den } \\
\text { Patienten inakzeptable Gefühle. }\end{array}$ & \\
\hline 66. ... beruhigt den P. & \\
\hline 89. ... stärkt die Abwehr. & \\
\hline $\begin{array}{l}\text { 79. ... spricht Veränderung in Stimmung / } \\
\text { Affekt in der Stunde an. }\end{array}$ & \\
\hline
\end{tabular}

\section{„Gesprächsverhalten“}

\begin{tabular}{|l|l|l|}
\hline 12. Schweigen & gehetztes Gespräch? & stockendes Gespräch? \\
\hline 3. Th erleichtert das Erzählen. & Hm, ja... & \\
\hline $\begin{array}{l}\text { 65. Th klarifiziert, formuliert neu oder } \\
\text { wiederholt mit anderen Worten. }\end{array}$ & Bragt Sichtweise an & Bestätigt Sichtweise \\
\hline $\begin{array}{l}\text { 99. Th fragt Sichtweise des P an. } \\
\text { 45. Th ist supportiv. }\end{array}$ & Bestärkt den P & Nimmt sie an \\
\hline $\begin{array}{l}\text { 42. Patient weist Bemerkungen des Th } \\
\text { zurück. }\end{array}$ & Weist zurück & \\
\hline
\end{tabular}




\section{„Besprochene Themen“}

\begin{tabular}{|c|c|c|}
\hline \multicolumn{3}{|l|}{ Spezifische Themen } \\
\hline \multicolumn{3}{|l|}{ 11. sexuelle Gefühle und Erfahrungen } \\
\hline \multicolumn{3}{|l|}{$\begin{array}{l}\text { 16. Körperfunktionen, Gesundheit, } \\
\text { körperliche Symptome }\end{array}$} \\
\hline \multicolumn{3}{|l|}{$\begin{array}{l}\text { 38. Aktivitäten, die P außerhalb der Sitzung } \\
\text { in Angriff nehmen soll }\end{array}$} \\
\hline \multicolumn{3}{|l|}{ 90. Träume und Phantasien des P. } \\
\hline \multicolumn{3}{|l|}{ 63. zwischenmenschliche Beziehungen } \\
\hline \multicolumn{3}{|l|}{ 64. Liebes- / romantische Beziehungen } \\
\hline \multicolumn{3}{|l|}{ 29. P.: will Trennung / Distanz } \\
\hline \multicolumn{3}{|l|}{ 33. P.: (Wunsch nach) Nähe } \\
\hline 69. Gegenwart vs. Vergangenheit (Min.) & G. & V. \\
\hline \multicolumn{3}{|l|}{ 91. Kindheit } \\
\hline $\begin{array}{l}\text { 92. Gefühle / Wahrnehmungen } \\
\text { mit Vergangenheit verknüpft }\end{array}$ & & \\
\hline
\end{tabular}

\section{Kontraktaspekte}

75. Unterbrechungen, Therapieende

96. Terminvereinbarungen, Honorar

\section{Ziele}

\begin{tabular}{|l|l|l|}
\hline 4. Therapieziele des P. & \multicolumn{2}{|l|}{} \\
\hline 41. Ziele, Vorhaben des P. & Vorhaben, Ziele & Einengung \\
\hline $\begin{array}{l}\text { 55. P.: positive Erwartungen bzgl. Therapie } \\
\text { 32. Der P. gewinnt neues Verständnis / } \\
\text { Einsicht }\end{array}$ & Ja & Negativ, pessimistisch \\
\hline
\end{tabular}

\section{Kognitive Themen}

\begin{tabular}{|l|l|l|}
\hline 30. Kognitive Themen allgemein & \multicolumn{2}{|l|}{} \\
\hline 35. Selbstbild & & \\
\hline $\begin{array}{l}\text { 68. Unterscheidung reale vs. phantasierte } \\
\text { Bedeutung von Erfahrung }\end{array}$ & Wahrnehmungsverzerrung? & Besprochen? \\
\hline
\end{tabular}




\section{„Therapeutische Interventionen“}

\begin{tabular}{|l|l|l|}
\hline 31. Th fragt nach mehr Information. & \multicolumn{2}{|l|}{} \\
\hline 27. Th gibt Ratschläge. & & Weigert sich \\
\hline $\begin{array}{l}\text { 57. Th erläutert das Rational. } \\
\text { 80. Th bietet Ereignis aus anderer } \\
\text { Perspektive an . }\end{array}$ & \\
\hline $\begin{array}{l}\text { 85. Th ermutigt zu neuen Verhaltens- } \\
\text { weisen im Umgang mit anderen. }\end{array}$ & \\
\hline $\begin{array}{l}\text { 48. Th ermutigt P zu Unabhängigkeit } \\
\text { im Handeln und Denken. }\end{array}$ & \\
\hline $\begin{array}{l}\text { 62. Th benennt ein sich wiederholendes } \\
\text { Thema. }\end{array}$ & \\
\hline $\begin{array}{l}\text { 2. Th benennt nonverbales Verhalten. } \\
\text { 36. Th weist auf Abwehrmechanismen } \\
\text { Hin. }\end{array}$ & \\
\hline
\end{tabular}

\section{„Therapeutische Interpretationen“}

\begin{tabular}{|l|l|l|}
\hline 40. ... bezogen auf konkrete Personen. & konkrete Personen & bezogen auf Patienten \\
\hline 43. ... das Verhalten anderer Personen. & \\
\hline 82. ... das Verhalten von P in der Stunde. & \\
\hline 67. ... unbewusstes Material. & \\
\hline
\end{tabular}

\section{„Thema Verantwortung“}

\begin{tabular}{|l|l|l|}
\hline $\begin{array}{l}\text { 34: P glaubt, für seine } \\
\text { Schwierigkeiten... }\end{array}$ & $\begin{array}{l}\text {...selbst verantwortlich } \mathrm{zu} \\
\text { sein, übernimmt } \\
\text { Verantwortung dafür. }\end{array}$ & $\begin{array}{l}\text { sind andere verantwortlich, } \\
\text { externalisiert. }\end{array}$ \\
\hline $\begin{array}{l}\text { 76: Wie reagiert der } \\
\text { Therapeut darauf? }\end{array}$ & $\begin{array}{l}\text { T sagt P, dass er schon } \mathrm{zu} \\
\text { viel Verantwortung } \\
\text { übernimmt. }\end{array}$ & $\begin{array}{l}\text { T legt P nahe, dass er handeln/ sich } \\
\text { verändern muss, wenn sich etwas } \\
\text { verbessern soll an seiner Situation. }\end{array}$ \\
\hline
\end{tabular}




\section{Anhang B: Ergebnisse der statistischen Analysen}

Tabelle 1: Itemmittelwerte und Standardabweichung der 10 charakteristischsten und 10 uncharakteristischsten Items bei der 10. und 18. Sitzung (gesamte Stichprobe).

\begin{tabular}{|c|c|c|c|c|c|c|}
\hline \multirow[t]{2}{*}{ PQS-Code } & \multicolumn{3}{|c|}{ 10. Sitzung } & \multicolumn{3}{|c|}{ 18. Sitzung } \\
\hline & Rang & $\mathbf{M}$ & sd & Rang & $\mathbf{M}$ & sd \\
\hline \multicolumn{7}{|c|}{ charakteristische Items } \\
\hline 3 & 1 & 8,56 & .78 & 1 & 8,55 & .72 \\
\hline 23 & 3 & 8,00 & .99 & 5 & 7,45 & 1,46 \\
\hline 30 & 8 & 7,14 & 1,57 & 8 & 7,11 & 1,23 \\
\hline 35 & 10 & 6,79 & 1,8 & 9 & 7,09 & 1,49 \\
\hline 46 & 7 & 7,24 & .79 & 7 & 7,28 & .70 \\
\hline 54 & 9 & 7,04 & 1,15 & 10 & 6,77 & 1,06 \\
\hline 65 & 4 & 7,95 & 1,33 & 3 & 8,06 & 1,21 \\
\hline 69 & 2 & 8,33 & .86 & 2 & 8,48 & .96 \\
\hline 80 & 6 & 7,42 & .84 & 4 & 7,46 & .86 \\
\hline 88 & 5 & 7,64 & .81 & 6 & 7,37 & .97 \\
\hline$\Sigma \mathrm{M}$ & & 76,11 & & & 75,62 & \\
\hline \multicolumn{7}{|c|}{ uncharakteristische Items } \\
\hline 2 & 99 & 1,31 & .54 & 99 & 1,45 & .68 \\
\hline 11 & 100 & 1,08 & .33 & 100 & 1,29 & .90 \\
\hline 29 & 94 & 2,23 & 1,50 & 93 & 2,44 & 1,48 \\
\hline 33 & 92 & 2,57 & 1,58 & 94 & 2,37 & 1,46 \\
\hline 36 & 95 & 2,13 & .93 & 95 & 2,01 & .93 \\
\hline 42 & 93 & 2,47 & .64 & 91 & 2,61 & .84 \\
\hline 49 & 96 & 2,01 & 1,40 & 96 & 1,67 & .65 \\
\hline 67 & 91 & 2,65 & 1,01 & 92 & 2,55 & .84 \\
\hline 90 & 97 & 1,77 & 1,29 & 98 & 1,54 & 1,0 \\
\hline 91 & 98 & 1,39 & .54 & 97 & 1,63 & 1,08 \\
\hline$\Sigma \mathrm{M}$ & & 19,61 & & & 20,56 & \\
\hline $\begin{array}{c}\Sigma \mathrm{M} \\
\text { (gesamt) }\end{array}$ & & 95,72 & & & 96,18 & \\
\hline
\end{tabular}

Anm: die fett gedruckten Items sind die, die nicht von Beginn an in beiden Teilstichproben (10. und 18. Sitzung) zu den jeweils 10 charakteristischsten bzw. uncharakteristischsten gehörten und die erst über die im Text beschriebene Vorgehensweise bestimmt wurden; PQS: Psychotherapy Process-Q-Sort, M: Mittelwert, sd: Standardabweichung. 
Anhang B

Tabelle 2: Ergebnisse der t-Tests zum Vergleich der 10. und 18. Sitzung (gesamte Stichprobe, df = 41).

\begin{tabular}{|c|c|c|c|c|c|}
\hline PQS-Code & $\mathbf{t}$ & $\mathbf{p}$ & PQS-Code & $\mathbf{t}$ & $\mathbf{p}$ \\
\hline 1 & 1,117 & .270 & 51 & $-1,001$ & .323 \\
\hline 2 & $-1,315$ & .196 & 52 & $-1,389$ & .172 \\
\hline 3 & .061 & .952 & 53 & 1,431 & .160 \\
\hline 4 & .862 & .394 & 54 & 1,144 & .259 \\
\hline 5 & -.003 & .998 & 55 & .000 & 1,000 \\
\hline 6 & .200 & .842 & 56 & -.166 & .869 \\
\hline 7 & .795 & .431 & 57 & .094 & .926 \\
\hline 8 & .384 & .703 & 58 & $-1,187$ & .242 \\
\hline 9 & -.267 & .791 & 59 & .479 & .635 \\
\hline 10 & -.834 & .409 & 60 & 2,009 & .051 \\
\hline 11 & $-1,496$ & .142 & 61 & .060 & .953 \\
\hline 12 & .585 & .562 & 62 & .979 & .333 \\
\hline 13 & -.791 & .434 & 63 & $-1,427$ & .161 \\
\hline 14 & -.968 & .339 & 64 & .204 & .839 \\
\hline 15 & -.774 & .443 & 65 & -.508 & .614 \\
\hline 16 & .240 & .811 & 66 & .495 & .623 \\
\hline 17 & .950 & .348 & 67 & .529 & .600 \\
\hline 18 & .198 & .844 & 68 & .553 & .583 \\
\hline 19 & .000 & 1,000 & 69 & -.944 & .351 \\
\hline 20 & -.751 & .457 & 70 & .724 & .473 \\
\hline 21 & $-2,196$ & .034 & 71 & $-1,633$ & .110 \\
\hline 22 & -.435 & .666 & 72 & .229 & .820 \\
\hline 23 & 2,295 & .027 & 73 & .472 & .639 \\
\hline 24 & .000 & 1,000 & 74 & $-2,128$ & .039 \\
\hline 25 & 1,550 & .129 & 75 & -.258 & .797 \\
\hline 26 & 1,084 & .285 & 76 & .140 & .890 \\
\hline 27 & -.871 & .389 & 77 & -.212 & .833 \\
\hline 28 & .881 & .383 & 78 & -.090 & .929 \\
\hline 29 & -.916 & .365 & 79 & -.361 & .720 \\
\hline 30 & .121 & .904 & 80 & -.202 & .841 \\
\hline 31 & .151 & .880 & 81 & .743 & .462 \\
\hline 32 & .202 & .841 & 82 & -.133 & .895 \\
\hline 33 & .882 & .383 & 83 & $-1,533$ & .133 \\
\hline 34 & -.539 & .593 & 84 & -.685 & .497 \\
\hline 35 & -.862 & .394 & 85 & $-1,937$ & .060 \\
\hline 36 & .687 & .496 & 86 & .728 & .471 \\
\hline 37 & 1,143 & .260 & 87 & $-2,053$ & .047 \\
\hline 38 & .999 & .324 & 88 & 1,5050 & .140 \\
\hline 39 & .635 & .529 & 89 & -.524 & .603 \\
\hline 40 & .744 & .461 & 90 & .925 & .360 \\
\hline 41 & -.479 & .634 & 91 & $-1,259$ & .215 \\
\hline 42 & -.837 & .407 & 92 & .324 & .747 \\
\hline 43 & .255 & .800 & 93 & $-1,087$ & .283 \\
\hline 44 & .078 & .938 & 94 & 1,794 & .080 \\
\hline 45 & -.163 & .871 & 95 & .385 & .702 \\
\hline 46 & -.275 & .785 & 96 & .290 & .774 \\
\hline 47 & 1,498 & .142 & 97 & .563 & .576 \\
\hline 48 & $-1,515$ & .137 & 98 & .688 & .495 \\
\hline 49 & 1,598 & .118 & 99 & -.382 & .705 \\
\hline 50 & -.122 & .904 & 100 & .276 & .784 \\
\hline
\end{tabular}


Tabelle 3: Itemmittelwerte und Standardabweichung der 10 charakteristischsten und 10 uncharakteristischsten Items bei der 10. und 18. Sitzung (psychodynamisch behandelte Stichprobe).

\begin{tabular}{|c|c|c|c|c|c|c|}
\hline \multicolumn{7}{|c|}{.Psychodynamische Therapien } \\
\hline \multirow{2}{*}{$\begin{array}{l}\text { PQS- } \\
\text { Code }\end{array}$} & \multicolumn{3}{|c|}{ 10. Sitzung } & \multicolumn{3}{|c|}{ 18. Sitzung } \\
\hline & Rang & $\mathbf{M}$ & sd & Rang & $\mathbf{M}$ & sd \\
\hline \multicolumn{7}{|c|}{ charakteristische Items } \\
\hline 3 & 2 & 8,34 & .87 & 1 & 8,39 & .77 \\
\hline 6 & 9 & 7,05 & .78 & 8 & 7,03 & .87 \\
\hline 23 & 4 & 7,81 & .93 & 4 & 7,41 & 1,82 \\
\hline 35 & 7 & 7,13 & 1,88 & 6 & 7,23 & 1,55 \\
\hline 46 & 10 & 6,89 & .72 & 9 & 6,98 & .53 \\
\hline 54 & 8 & 7,10 & 1,05 & 10 & 6,63 & .87 \\
\hline 65 & 1 & 8,53 & .81 & 2 & 8,38 & .67 \\
\hline 69 & 3 & 8,19 & .92 & 3 & 8,36 & .95 \\
\hline 80 & 6 & 7,38 & .98 & 5 & 7,40 & .93 \\
\hline 88 & 5 & 7,50 & .82 & 7 & 7,11 & 1,15 \\
\hline$\Sigma \mathrm{M}$ & & 75,92 & & & 74,92 & \\
\hline \multicolumn{7}{|c|}{ uncharakteristische Items } \\
\hline 2 & 99 & 1,18 & .34 & 99 & 1,34 & .62 \\
\hline 11 & 100 & 1,13 & .47 & 100 & 1,34 & .62 \\
\hline 29 & 94 & 2,34 & 1,56 & 93 & 2,55 & 1,64 \\
\hline 33 & 92 & 2,80 & 2,02 & 94 & 2,82 & 1,96 \\
\hline 36 & 95 & 2,43 & 1,13 & 95 & 2,40 & 1,19 \\
\hline 38 & 93 & 2,49 & 2,01 & 91 & 2,71 & 2,16 \\
\hline 42 & 96 & 2,45 & .72 & 96 & 2,74 & 1,03 \\
\hline 49 & 91 & 2,56 & 1,91 & 92 & 1,90 & .77 \\
\hline 90 & 97 & 2,00 & 1,83 & 98 & 1,59 & .63 \\
\hline 91 & 98 & 1,45 & .57 & 97 & 1,75 & 1,51 \\
\hline$\Sigma \mathrm{M}$ & & 21,83 & & & 21,14 & \\
\hline $\begin{array}{c}\Sigma \mathrm{M} \\
\text { (gesamt) }\end{array}$ & & 97,75 & & & 96,06 & \\
\hline
\end{tabular}

Anm: die fett gedruckten Items sind die, die nicht von Beginn an in beiden Teilstichproben (10. und 18. Sitzung) zu den jeweils 10 charakteristischsten bzw. uncharakteristischsten gehörten und die erst über die im Text beschriebene Vorgehensweise bestimmt wurden; PQS: Psychotherapy Process-Q-Sort; M: Mittelwert, sd: Standardabweichung. 
Tabelle 4: Ergebnisse der t-Tests zum Vergleich der 10. und der 18. Sitzung (psychodynamische Therapie, df = 18).

\begin{tabular}{|c|c|c|c|c|c|}
\hline PQS-Code & $t$ & $\mathbf{p}$ & PQS-Code & $t$ & $\mathbf{p}$ \\
\hline 1 & .893 & .384 & 51 & $-1,909$ & .072 \\
\hline 2 & -.972 & .344 & 52 & $-1,672$ & .112 \\
\hline 3 & -.276 & .786 & 53 & 2,592 & .018 \\
\hline 4 & .524 & .607 & 54 & 1,401 & .178 \\
\hline 5 & .374 & .713 & 55 & .653 & .522 \\
\hline 6 & .114 & .910 & 56 & .213 & .834 \\
\hline 7 & .277 & .785 & 57 & -.743 & .467 \\
\hline 8 & -.266 & .839 & 58 & $-1,048$ & .308 \\
\hline 9 & -.188 & .853 & 59 & .645 & .527 \\
\hline 10 & .192 & .850 & 60 & 1,392 & .181 \\
\hline 11 & $-1,443$ & .166 & 61 & .323 & .751 \\
\hline 12 & .574 & .573 & 62 & .128 & .899 \\
\hline 13 & -.375 & .712 & 63 & $-1,269$ & .220 \\
\hline 14 & $-2,075$ & .053 & 64 & -.054 & .958 \\
\hline 15 & $-1,239$ & .231 & 65 & .647 & .526 \\
\hline 16 & -.532 & .601 & 66 & 677 & .507 \\
\hline 17 & .883 & .389 & 67 & .279 & .784 \\
\hline 18 & .721 & .480 & 68 & .221 & .827 \\
\hline 19 & .567 & .578 & 69 & -.759 & .458 \\
\hline 20 & -.510 & .617 & 70 & -.350 & .731 \\
\hline 21 & $-1,190$ & .249 & 71 & -.456 & .654 \\
\hline 22 & $-1,068$ & .300 & 72 & .497 & .625 \\
\hline 23 & .991 & .335 & 73 & .777 & .447 \\
\hline 24 & .000 & 1,000 & 74 & -.950 & .355 \\
\hline 25 & 2,003 & .060 & 75 & -.533 & .600 \\
\hline 26 & .160 & .875 & 76 & - .724 & .478 \\
\hline 27 & $-1,216$ & .240 & 77 & -.914 & .373 \\
\hline 28 & .688 & .500 & 78 & .728 & .476 \\
\hline 29 & -.461 & .650 & 79 & $-1,811$ & .087 \\
\hline 30 & .507 & .618 & 80 & -.081 & .936 \\
\hline 31 & -.071 & .944 & 81 & 1,747 & .098 \\
\hline 32 & .389 & .702 & 82 & -.029 & .977 \\
\hline 33 & -.064 & .950 & 83 & -.901 & .379 \\
\hline 34 & $-1,235$ & .233 & 84 & .515 & .613 \\
\hline 35 & -.216 & .832 & 85 & $-1,071$ & .298 \\
\hline 36 & .096 & .924 & 86 & -.079 & .938 \\
\hline 37 & -.275 & .786 & 87 & $-2,328$ & .032 \\
\hline 38 & -.478 & .638 & 88 & 1,400 & .178 \\
\hline 39 & -.237 & .816 & 89 & -.991 & .335 \\
\hline 40 & .722 & .480 & 90 & .979 & .341 \\
\hline 41 & -.658 & .519 & 91 & -.860 & .401 \\
\hline 42 & $-1,009$ & .326 & 92 & 1,247 & .228 \\
\hline 43 & .964 & .348 & 93 & 1,106 & .283 \\
\hline 44 & -.002 & .999 & 94 & 1,271 & .220 \\
\hline 45 & .093 & .927 & 95 & 1,520 & .146 \\
\hline 46 & -.373 & .714 & 96 & .044 & .966 \\
\hline 47 & 1,680 & .110 & 97 & 1,297 & .211 \\
\hline 48 & -.817 & .425 & 98 & .684 & .503 \\
\hline 49 & 1,535 & .142 & 99 & .065 & .949 \\
\hline 50 & -.507 & .618 & 100 & 1,027 & .318 \\
\hline
\end{tabular}


Tabelle 5: Itemmittelwerte der 10 charakteristischsten und 10 uncharakteristischsten Items bei der 10. und 18. Sitzung (kognitiv-behavioral behandelte Stichprobe).

\begin{tabular}{|c|c|c|c|c|c|c|}
\hline \multicolumn{7}{|c|}{ Kognitiv-behaviorale Therapien } \\
\hline \multirow{2}{*}{$\begin{array}{l}\text { PQS- } \\
\text { Code }\end{array}$} & \multicolumn{3}{|c|}{ 10. Sitzung } & \multicolumn{3}{|c|}{ 18. Sitzung } \\
\hline & Rang & $\mathbf{M}$ & sd & Rang & $\mathbf{M}$ & sd \\
\hline \multicolumn{7}{|c|}{ charakteristische Items } \\
\hline 3 & 1 & 8,73 & .66 & 1 & 8,67 & .67 \\
\hline 23 & 3 & 8,16 & 1,03 & 8 & 7,48 & 1,11 \\
\hline 30 & 6 & 7,51 & 1,49 & 4 & 7,61 & .95 \\
\hline 45 & 9 & 7,25 & 1,07 & 9 & 7,35 & 1,50 \\
\hline 46 & 5 & 7,53 & .73 & 6 & 7,52 & .75 \\
\hline 65 & 7 & 7,47 & 1,50 & 3 & 7,80 & 1,48 \\
\hline 69 & 2 & 8,43 & .80 & 2 & 8,59 & .67 \\
\hline 72 & 10 & 7,05 & .63 & 10 & 7,11 & .84 \\
\hline 80 & 8 & 7,46 & .72 & 7 & 7,50 & .83 \\
\hline 88 & 4 & 7,76 & .81 & 5 & 7,59 & .73 \\
\hline$\Sigma \mathrm{M}$ & & 77,35 & & & 77,22 & \\
\hline \multicolumn{7}{|c|}{ uncharakteristische Items } \\
\hline 2 & 98 & 1,41 & .66 & 96 & 1,54 & .72 \\
\hline 11 & 100 & 1,04 & .14 & 100 & 1,07 & .17 \\
\hline 29 & 94 & 2,14 & 1,48 & 92 & 2,35 & 1,36 \\
\hline 33 & 92 & 2,38 & 1,10 & 94 & 2,00 & .71 \\
\hline 36 & 95 & 1,89 & .66 & 95 & 1,70 & .49 \\
\hline 42 & 91 & 2,49 & .57 & 91 & 2,50 & .66 \\
\hline 49 & 97 & 1,55 & .42 & 99 & 1,48 & .46 \\
\hline 67 & 93 & 2,28 & .90 & 93 & 2,17 & .42 \\
\hline 90 & 96 & 1,58 & .54 & 98 & 1,50 & 1,24 \\
\hline 91 & 99 & 1,35 & .51 & 97 & 1,52 & .53 \\
\hline$\Sigma \mathrm{M}$ & & 18,11 & & & 17,84 & \\
\hline $\begin{array}{c}\Sigma \mathrm{M} \\
\text { (gesamt) }\end{array}$ & & 95,46 & & & 95,06 & \\
\hline
\end{tabular}

Anm: die fett gedruckten Items sind die, die nicht von Beginn an in beiden Teilstichproben (10. und 18. Sitzung) zu den jeweils 10 charakteristischsten bzw. uncharakteristischsten gehörten und die erst über die im Text beschriebene Vorgehensweise bestimmt wurden; PQS: Psychotherapy Process-Q-Sort; M: Mittelwert, sd: Standardabweichung. 
Anhang B

Tabelle 6: Ergebnisse der t-Tests zum Vergleich der 10. und 18. Sitzung (kognitiv-behaviorale Therapie, $\mathrm{df}=$ 22).

\begin{tabular}{|c|c|c|c|c|c|}
\hline PQS-Code & $t$ & $\mathbf{p}$ & PQS-Code & $\mathbf{t}$ & $\mathbf{p}$ \\
\hline 1 & .670 & .510 & 51 & .118 & 907 \\
\hline 2 & -.879 & .389 & 52 & -.375 & .711 \\
\hline 3 & .318 & .754 & 53 & -.576 & .570 \\
\hline 4 & .672 & .508 & 54 & .310 & .759 \\
\hline 5 & -.189 & .852 & 55 & -.858 & .400 \\
\hline 6 & .162 & .873 & 56 & -.419 & .679 \\
\hline 7 & .761 & .454 & 57 & .548 & .589 \\
\hline 8 & .600 & .555 & 58 & -.552 & .586 \\
\hline 9 & -.186 & .854 & 59 & .043 & .966 \\
\hline 10 & $-1,521$ & .142 & 60 & 1,417 & .171 \\
\hline 11 & -.569 & .575 & 61 & -.267 & .792 \\
\hline 12 & .196 & .847 & 62 & 1,083 & .291 \\
\hline 13 & -.742 & .466 & 63 & -.735 & .470 \\
\hline 14 & .599 & .555 & 64 & -.223 & .826 \\
\hline 15 & .055 & .956 & 65 & -.909 & .373 \\
\hline 16 & .665 & .513 & 66 & -.058 & .954 \\
\hline 17 & .391 & .699 & 67 & .494 & .626 \\
\hline 18 & -.379 & .708 & 68 & .574 & .572 \\
\hline 19 & -.569 & .575 & 69 & -.603 & .553 \\
\hline 20 & -.554 & .585 & 70 & 1,341 & .194 \\
\hline 21 & $-1,196$ & .063 & 71 & $-1,858$ & .277 \\
\hline 22 & .450 & .657 & 72 & -.268 & .791 \\
\hline 23 & 2,303 & .031 & 73 & -.131 & .897 \\
\hline 24 & .000 & 1,000 & 74 & $-2,020$ & .056 \\
\hline 25 & .031 & .9760 & 75 & .185 & .855 \\
\hline 26 & 1,125 & .234 & 76 & .757 & .457 \\
\hline 27 & .238 & .814 & 77 & .585 & .564 \\
\hline 28 & .699 & .492 & 78 & -.787 & .441 \\
\hline 29 & $-1,075$ & .294 & 79 & .810 & .426 \\
\hline 30 & -.248 & .806 & 80 & -.234 & .817 \\
\hline 31 & .252 & .804 & 81 & -.421 & .678 \\
\hline 32 & -.065 & .948 & 82 & -.336 & .740 \\
\hline 33 & 1,685 & .106 & 83 & $-1,237$ & .229 \\
\hline 34 & .442 & .663 & 84 & $-1,913$ & .069 \\
\hline 35 & -.893 & .381 & 85 & $-1,644$ & .114 \\
\hline 36 & 1,335 & .196 & 86 & -.987 & .334 \\
\hline 37 & 2,033 & .054 & 87 & -.692 & .496 \\
\hline 38 & 1,649 & .113 & 88 & .723 & .477 \\
\hline 39 & 1,239 & .228 & 89 & .135 & .894 \\
\hline 40 & .341 & .736 & 90 & .268 & .791 \\
\hline 41 & -.064 & .950 & 91 & -.984 & .336 \\
\hline 42 & -.076 & .940 & 92 & $-1,521$ & .142 \\
\hline 43 & -.548 & .589 & 93 & -.295 & .770 \\
\hline 44 & .121 & .905 & 94 & 1,236 & .229 \\
\hline 45 & -.315 & .755 & 95 & -.985 & .335 \\
\hline 46 & .046 & .964 & 96 & .324 & .749 \\
\hline 47 & .480 & .636 & 97 & -.333 & .742 \\
\hline 48 & $-1,282$ & .213 & 98 & -.188 & .853 \\
\hline 49 & .507 & .617 & 99 & -.716 & .481 \\
\hline 50 & .413 & .684 & 100 & -.768 & .451 \\
\hline
\end{tabular}


Tabelle 7: Vergleich der 10 charakteristischsten und 10 uncharakteristischsten Items in der psychodynamisch und kognitiv-behavioral behandelten Stichprobe.

\begin{tabular}{|c|c|c|c|c|c|c|c|}
\hline \multicolumn{4}{|c|}{$\begin{array}{l}\text { Psychodynamische Therapie } \\
\qquad(\mathrm{n}=19)\end{array}$} & \multicolumn{4}{|c|}{$\begin{array}{l}\text { Kognitiv-behaviorale Therapie } \\
(\mathbf{n}=23)\end{array}$} \\
\hline $\begin{array}{l}\text { PQS- } \\
\text { Code }\end{array}$ & Rang & $\mathbf{M}$ & sd & $\begin{array}{l}\text { PQS- } \\
\text { Code }\end{array}$ & Rang & $\mathbf{M}$ & sd \\
\hline \multicolumn{8}{|c|}{ Charakteristische Items } \\
\hline 3 & 2 & 8,37 & .71 & 3 & 1 & 8,70 & .50 \\
\hline 6 & 8 & 7,04 & .75 & 23 & 3 & 7,82 & .80 \\
\hline 23 & 4 & 7,61 & 1,15 & 30 & 6 & 7,56 & .85 \\
\hline 35 & 7 & 7,18 & 1,41 & 45 & 9 & 7,30 & 1,05 \\
\hline 46 & 9 & 6,93 & .29 & 46 & 7 & 7,53 & .63 \\
\hline 54 & 10 & 6,86 & .64 & 65 & 5 & 7,64 & 1,21 \\
\hline 65 & 1 & 8,45 & .55 & 69 & 2 & 8,51 & .65 \\
\hline 69 & 3 & 8,28 & .80 & 72 & 10 & 7,08 & .53 \\
\hline 80 & 5 & 7,39 & .63 & 80 & 8 & 7,48 & .63 \\
\hline 88 & 6 & 7,30 & .79 & 88 & 4 & 7,67 & .51 \\
\hline$\Sigma \mathrm{M}$ & & 75,41 & & & & 77,29 & \\
\hline \multicolumn{8}{|c|}{ Uncharakteristische Items } \\
\hline 2 & & 2,26 & .36 & 2 & 8 & 1,47 & .58 \\
\hline 11 & & 1,34 & .74 & 11 & 10 & 1,05 & .13 \\
\hline 29 & & 2,45 & 1,25 & 29 & 2 & 2,24 & 1,34 \\
\hline 33 & & 2,81 & 1,77 & 33 & 4 & 2,19 & .75 \\
\hline 36 & & 2,41 & .85 & 36 & 5 & 1,79 & .46 \\
\hline 38 & & 2,60 & 1,83 & 42 & 1 & 2,49 & .41 \\
\hline 42 & & 2,59 & .64 & 49 & 7 & 1,51 & .28 \\
\hline 49 & & 2,23 & 1,12 & 67 & 3 & 2,23 & .46 \\
\hline 90 & & 1,79 & 1,02 & 90 & 6 & 1,54 & .64 \\
\hline 91 & & 1,60 & .84 & 91 & 9 & 1,43 & .30 \\
\hline$\Sigma \mathrm{M}$ & & 22,08 & & & & 17,94 & \\
\hline $\begin{array}{c}\Sigma \mathrm{M} \\
\text { (gesamt) }\end{array}$ & & 97,49 & & & & 95,23 & \\
\hline
\end{tabular}

Anm: die fett gedruckten Items sind die, die nicht in beiden Teilstichproben (psychodynamische und kognitiv-behaviorale Sitzungen) zu den jeweils 10 charakteristischsten bzw. uncharakteristischsten gehören; PQS: Psychotherapy Process-QSort; M: Mittelwert, sd: Standardabweichung. 
Tabelle 8: Ergebnisse der linearen Regressionen für die gesamte Stichprobe für die HAMA.

\begin{tabular}{|c|c|c|c|c|}
\hline Rang & PQS-Code & $\mathbf{R}^{2}$ & $\mathbf{p}$ & 及 \\
\hline 1 & 3 & .004 & .679 & .067 \\
\hline 2 & 69 & .039 & .218 & .197 \\
\hline 3 & 65 & .001 & .811 & .038 \\
\hline 4 & 23 & .002 & .772 & -.047 \\
\hline 5 & 88 & .001 & .883 & -.024 \\
\hline 6 & 80 & .024 & .337 & -.154 \\
\hline 7 & 46 & .003 & .743 & .053 \\
\hline 8 & 30 & .012 & .495 & -.110 \\
\hline 9 & 35 & .003 & .721 & -.057 \\
\hline 10 & 54 & .000 & .961 & -.008 \\
\hline 91 & 67 & .060 & .123 & -.245 \\
\hline 92 & 42 & .052 & .151 & .228 \\
\hline 93 & 33 & .031 & .271 & -.176 \\
\hline 94 & 29 & .050 & .161 & .223 \\
\hline 95 & 36 & .037 & .231 & -.191 \\
\hline 96 & 49 & .018 & .400 & -.135 \\
\hline 97 & 90 & .000 & .970 & -.006 \\
\hline 98 & 91 & .027 & .300 & -.166 \\
\hline 99 & 2 & .031 & .271 & .176 \\
\hline 100 & 11 & .005 & .668 & -.069 \\
\hline
\end{tabular}

Tabelle 9: Ergebnisse der linearen Regressionen für die kognitiv-behavioral behandelte Stichprobe für die HAMA.

\begin{tabular}{|c|c|c|c|c|}
\hline Rang & PQS-Code & $\mathbf{R}^{\mathbf{2}}$ & $\mathbf{p}$ & 及 \\
\hline 1 & 3 & .068 & .240 & .261 \\
\hline 2 & 69 & .004 & .771 & -.066 \\
\hline 3 & 23 & .015 & .591 & -.121 \\
\hline 4 & 88 & .001 & .895 & .030 \\
\hline 5 & 65 & .045 & .342 & .213 \\
\hline 6 & 30 & .120 & .114 & -.346 \\
\hline 7 & 46 & .004 & .790 & -.060 \\
\hline 8 & 80 & .000 & .939 & -.017 \\
\hline 9 & 45 & .006 & .737 & -.076 \\
\hline 10 & 72 & .015 & .588 & -.122 \\
\hline 91 & 42 & .009 & .668 & .097 \\
\hline 92 & 29 & .148 & .077 & .385 \\
\hline 93 & 67 & .000 & .983 & .005 \\
\hline 94 & 33 & .009 & .673 & -.095 \\
\hline 95 & 36 & .006 & .732 & .077 \\
\hline 96 & 90 & .025 & .484 & .157 \\
\hline 97 & 49 & .000 & .991 & -.003 \\
\hline 98 & 2 & .001 & .946 & .033 \\
\hline 99 & 91 & .007 & .713 & .083 \\
\hline 100 & 11 & .000 & .737 & .015 \\
\hline
\end{tabular}


Tabelle 10: Ergebnisse der linearen Regressionen für die psychodynamisch behandelte Stichprobe für die HAMA.

\begin{tabular}{|c|c|c|c|c|}
\hline Rang & PQS-Code & $\mathbf{R}^{\mathbf{2}}$ & $\mathbf{p}$ & 及 \\
\hline 1 & 65 & .069 & .278 & -.262 \\
\hline 2 & 3 & .046 & .377 & -.215 \\
\hline 3 & 69 & .246 & .031 & .496 \\
\hline 4 & 23 & .000 & .951 & -.015 \\
\hline 5 & 80 & .151 & .100 & -.389 \\
\hline 6 & 88 & .030 & .479 & -.173 \\
\hline 7 & 35 & .004 & .788 & -.066 \\
\hline 8 & 6 & .257 & .027 & .092 \\
\hline 9 & 46 & .008 & .707 & .092 \\
\hline 10 & 54 & .030 & .477 & .174 \\
\hline 91 & 33 & .046 & .377 & -.215 \\
\hline 92 & 38 & .197 & .057 & .444 \\
\hline 93 & 42 & .184 & .067 & .429 \\
\hline 94 & 29 & .000 & .947 & .016 \\
\hline 95 & 36 & .128 & .133 & -.358 \\
\hline 96 & 49 & .021 & .555 & -.144 \\
\hline 97 & 90 & .015 & .621 & -.121 \\
\hline 98 & 91 & .104 & .178 & -.322 \\
\hline 99 & 11 & .003 & .810 & -.059 \\
\hline 100 & 2 & .201 & .054 & .448 \\
\hline
\end{tabular}

Tabelle 11: Ergebnisse der linearen Regressionen für die gesamte Stichprobe für die HAMD.

\begin{tabular}{|c|c|c|c|c|}
\hline Rang & PQS-Code & $\mathbf{R}^{2}$ & $\mathbf{p}$ & 及 \\
\hline 1 & 3 & .027 & .308 & .163 \\
\hline 2 & 69 & .001 & .873 & .026 \\
\hline 3 & 65 & .005 & .656 & .072 \\
\hline 4 & 23 & .001 & .840 & -.032 \\
\hline 5 & 88 & .005 & .669 & .069 \\
\hline 6 & 80 & .000 & .955 & -.009 \\
\hline 7 & 46 & .006 & .643 & .075 \\
\hline 8 & 30 & .060 & .124 & .244 \\
\hline 9 & 35 & .025 & .326 & .157 \\
\hline 10 & 54 & .003 & .733 & .055 \\
\hline 91 & 67 & .019 & .391 & -.138 \\
\hline 92 & 42 & .089 & .058 & .299 \\
\hline 93 & 33 & .059 & .126 & -.243 \\
\hline 94 & 29 & .000 & .913 & -.018 \\
\hline 95 & 36 & .027 & .306 & -.164 \\
\hline 96 & 49 & .089 & .057 & -.299 \\
\hline 97 & 90 & .030 & .275 & .175 \\
\hline 98 & 91 & .007 & .591 & -.087 \\
\hline 99 & 2 & .000 & .926 & -.015 \\
\hline 100 & 11 & .009 & .560 & -.094 \\
\hline
\end{tabular}


Tabelle 12: Ergebnisse der linearen Regressionen für die psychodynamisch behandelte Stichprobe für die HAMD.

\begin{tabular}{|c|c|c|c|c|}
\hline Rang & PQS-Code & $\mathbf{R}^{2}$ & $\mathbf{p}$ & 及 \\
\hline 1 & 65 & .053 & .343 & -.230 \\
\hline 2 & 3 & .017 & .590 & -.132 \\
\hline 3 & 69 & .088 & .219 & .296 \\
\hline 4 & 23 & .013 & .645 & -.113 \\
\hline 5 & 80 & .009 & .702 & .094 \\
\hline 6 & 88 & .016 & .611 & -.125 \\
\hline 7 & 35 & .091 & .209 & .302 \\
\hline 8 & 6 & .060 & .310 & -.246 \\
\hline 9 & 46 & .192 & .061 & .438 \\
\hline 10 & 54 & .023 & .536 & .151 \\
\hline 91 & 33 & .047 & .372 & -.217 \\
\hline 92 & 38 & .002 & .859 & .044 \\
\hline 93 & 42 & .123 & .142 & .350 \\
\hline 94 & 29 & .064 & .295 & -.254 \\
\hline 95 & 36 & .014 & .624 & -.120 \\
\hline 96 & 49 & .141 & .114 & -.375 \\
\hline 97 & 90 & .132 & .126 & .364 \\
\hline 98 & 91 & .015 & .622 & -.121 \\
\hline 99 & 11 & .000 & .961 & -.012 \\
\hline 100 & 2 & .011 & .674 & -.103 \\
\hline
\end{tabular}

Tabelle 13: Ergebnisse der linearen Regressionen für die kognitiv-behavioral behandelte Stichprobe für die HAMD.

\begin{tabular}{|c|c|c|c|c|}
\hline Rang & PQS-Code & $\mathbf{R}^{2}$ & $\mathbf{p}$ & 及 \\
\hline 1 & 3 & .088 & .179 & .297 \\
\hline 2 & 69 & .054 & .298 & -.232 \\
\hline 3 & 23 & .002 & .843 & -.045 \\
\hline 4 & 88 & .008 & .691 & .090 \\
\hline 5 & 65 & .096 & .160 & .310 \\
\hline 6 & 30 & .001 & .874 & -.036 \\
\hline 7 & 46 & .046 & .339 & -.214 \\
\hline 8 & 80 & .008 & .685 & -.092 \\
\hline 9 & 45 & .004 & .780 & -063 \\
\hline 10 & 72 & .047 & .333 & .217 \\
\hline 91 & 42 & .162 & .063 & .402 \\
\hline 92 & 29 & .024 & .488 & .156 \\
\hline 93 & 67 & .006 & .742 & .075 \\
\hline 94 & 33 & .059 & .276 & -.243 \\
\hline 95 & 36 & .000 & .987 &. .004 \\
\hline 96 & 90 & .018 & .549 & .135 \\
\hline 97 & 49 & .007 & .716 & -.082 \\
\hline 98 & 2 & .005 & .688 & .068 \\
\hline 99 & 91 & .000 & .955 & -.091 \\
\hline 100 & 11 & .008 & .688 & \\
\hline
\end{tabular}


Tabelle 14: Ergebnisse der linearen Regressionen für die gesamte Stichprobe für das BAI.

\begin{tabular}{|c|c|c|c|c|}
\hline Rang & PQS-Code & $\mathbf{R}^{\mathbf{2}}$ & $\mathbf{p}$ & 及 \\
\hline 1 & 3 & .068 & .096 & .261 \\
\hline 2 & 69 & .000 & .312 & -.017 \\
\hline 3 & 65 & .083 & .064 & .288 \\
\hline 4 & 23 & .057 & .129 & -.238 \\
\hline 5 & 88 & .065 & .103 & -.255 \\
\hline 6 & 80 & .033 & .250 & .182 \\
\hline 7 & 46 & .059 & .121 & .243 \\
\hline 8 & 30 & .018 & .402 & -.133 \\
\hline 9 & 35 & .009 & .557 & -.093 \\
\hline 10 & 54 & .123 & .023 & -.350 \\
\hline 91 & 67 & .065 & .102 & -.256 \\
\hline 92 & 42 & .002 & .754 & .050 \\
\hline 93 & 33 & .107 & .034 & -.328 \\
\hline 94 & 29 & .003 & .732 & -.055 \\
\hline 95 & 36 & .000 & .955 & -.009 \\
\hline 96 & 49 & .007 & .599 & -.083 \\
\hline 97 & 90 & .003 & .712 & -.059 \\
\hline 98 & 91 & .004 & .698 & -.062 \\
\hline 99 & 2 & .131 & .019 & .362 \\
\hline 100 & 11 & .004 & .707 & -.060 \\
\hline
\end{tabular}

Tabelle 15: Ergebnisse der linearen Regressionen für die psychodynamisch behandelte Stichprobe für das BAI.

\begin{tabular}{|c|c|c|c|c|}
\hline Rang & PQS-Code & $\mathbf{R}^{\mathbf{2}}$ & $\mathbf{p}$ & 及 \\
\hline 1 & 65 & .039 & .419 & .197 \\
\hline 2 & 3 & .268 & .023 & .518 \\
\hline 3 & 69 & .000 & .978 & .007 \\
\hline 4 & 23 & .093 & .205 & -.304 \\
\hline 5 & 80 & .007 & .737 & .083 \\
\hline 6 & 88 & .142 & .112 & -.376 \\
\hline 7 & 35 & .000 & .928 & -.022 \\
\hline 8 & 6 & .000 & .984 & -.005 \\
\hline 9 & 46 & .065 & .291 & .256 \\
\hline 10 & 54 & .000 & .959 & .013 \\
\hline 91 & 33 & .133 & .125 & -.364 \\
\hline 92 & 38 & .019 & .576 & -.137 \\
\hline 93 & 42 & .119 & .148 & .345 \\
\hline 94 & 29 & .008 & .709 & .092 \\
\hline 95 & 36 & .004 & .787 & .066 \\
\hline 96 & 49 & .005 & .779 & -.069 \\
\hline 97 & 90 & .006 & .747 & -.079 \\
\hline 98 & 91 & .030 & .476 & -.174 \\
\hline 99 & 11 & .002 & .864 & -.042 \\
\hline 100 & 2 & .041 & .405 & -.203 \\
\hline
\end{tabular}


Tabelle 16: Ergebnisse der linearen Regressionen für die kognitiv-behavioral behandelte Stichprobe für das BAI.

\begin{tabular}{|c|c|c|c|c|}
\hline Rang & PQS-Code & $\mathbf{R}^{\mathbf{2}}$ & $\mathbf{p}$ & 及 \\
\hline 1 & 3 & .003 & .816 & .051 \\
\hline 2 & 69 & .005 & .746 & -.072 \\
\hline 3 & 23 & .062 & .250 & -.250 \\
\hline 4 & 88 & .086 & .175 & -.293 \\
\hline 5 & 65 & .175 & .047 & .418 \\
\hline 6 & 30 & .138 & .081 & -.371 \\
\hline 7 & 46 & .041 & .355 & .202 \\
\hline 8 & 80 & .053 & .292 & .230 \\
\hline 9 & 45 & .006 & .734 & .075 \\
\hline 10 & 72 & .270 & .011 & -.520 \\
\hline 91 & 42 & .027 & .458 & -.163 \\
\hline 92 & 29 & .013 & .600 & -.115 \\
\hline 93 & 67 & .040 & .362 & -.199 \\
\hline 94 & 33 & .148 & .070 & -.384 \\
\hline 95 & 36 & .002 & .839 & .045 \\
\hline 96 & 90 & .000 & .949 & -.014 \\
\hline 97 & 49 & .000 & .999 & .000 \\
\hline 98 & 2 & .282 & .009 & .531 \\
\hline 99 & 91 & .014 & .590 & .119 \\
\hline 100 & 11 & .001 & .885 & -.032 \\
\hline
\end{tabular}

Tabelle 17: Ergebnisse der linearen Regressionen für die gesamte Stichprobe für das BDI.

\begin{tabular}{|c|c|c|c|c|}
\hline Rang & PQS-Code & $\mathbf{R}^{\mathbf{2}}$ & $\mathbf{p}$ & $\mathbf{B}$ \\
\hline 1 & 3 & .001 & .821 & .036 \\
\hline 2 & 69 & .029 & .285 & .169 \\
\hline 3 & 65 & .014 & .460 & -.117 \\
\hline 4 & 23 & .012 & .485 & -.111 \\
\hline 5 & 88 & .011 & .521 & -.104 \\
\hline 6 & 80 & .008 & .574 & .089 \\
\hline 7 & 46 & .007 & .598 & -.084 \\
\hline 8 & 30 & .001 & .853 & .030 \\
\hline 9 & 35 & .008 & .577 & -.089 \\
\hline 10 & 54 & .029 & .278 & .171 \\
\hline 91 & 67 & .018 & .397 & -.134 \\
\hline 92 & 42 & .033 & .256 & .183 \\
\hline 93 & 33 & .039 & .209 & -.198 \\
\hline 94 & 29 & .001 & .846 & .031 \\
\hline 95 & 36 & .000 & .972 & .005 \\
\hline 96 & 49 & .088 & .057 & -.296 \\
\hline 97 & 90 & .004 & .681 & -.065 \\
\hline 98 & 91 & .024 & .330 & -.154 \\
\hline 99 & 2 & .008 & .568 & .091 \\
\hline 100 & 11 & .000 & .980 & -.004 \\
\hline
\end{tabular}


Tabelle 18: Ergebnisse der linearen Regressionen für die psychodynamisch behandelte Stichprobe für das BDI.

\begin{tabular}{|c|c|c|c|c|}
\hline Rang & PQS-Code & $\mathbf{R}^{\mathbf{2}}$ & $\mathbf{p}$ & 及 \\
\hline 1 & 65 & .009 & .696 & -.096 \\
\hline 2 & 3 & .007 & .734 & .083 \\
\hline 3 & 69 & .024 & .526 & .155 \\
\hline 4 & 23 & .039 & .421 & -.196 \\
\hline 5 & 80 & .008 & .717 & -.089 \\
\hline 6 & 88 & .023 & .534 & -.152 \\
\hline 7 & 35 & .006 & .744 & .080 \\
\hline 8 & 6 & .055 & .335 & -.234 \\
\hline 9 & 46 & .132 & .126 & .364 \\
\hline 10 & 54 & .126 & .135 & .355 \\
\hline 91 & 33 & .042 & .398 & -.206 \\
\hline 92 & 38 & .022 & .549 & .147 \\
\hline 93 & 42 & .085 & .226 & .292 \\
\hline 94 & 29 & .013 & .282 & -.260 \\
\hline 95 & 36 & .005 & .785 & .067 \\
\hline 96 & 49 & .071 & .271 & -.266 \\
\hline 97 & 90 & .001 & .886 & .035 \\
\hline 98 & 91 & .022 & .542 & -.149 \\
\hline 99 & 11 & .025 & .516 & .159 \\
\hline 100 & 2 & .017 & .599 & -.129 \\
\hline
\end{tabular}

Tabelle 19: Ergebnisse der linearen Regressionen für die kognitiv-behavioral behandelte Stichprobe für das BDI.

\begin{tabular}{|c|c|c|c|c|}
\hline Rang & PQS-Code & $\mathbf{R}^{\mathbf{2}}$ & $\mathbf{p}$ & 及 \\
\hline 1 & 3 & .036 & .386 & -.190 \\
\hline 2 & 69 & .011 & .628 & .107 \\
\hline 3 & 23 & .013 & .604 & -.114 \\
\hline 4 & 88 & .079 & .194 & -.281 \\
\hline 5 & 65 & .001 & .878 & .034 \\
\hline 6 & 30 & .165 & .054 & -.406 \\
\hline 7 & 46 & .268 & .011 & -.518 \\
\hline 8 & 80 & .035 & 395 & .186 \\
\hline 9 & 45 & .079 & .193 & .282 \\
\hline 10 & 72 & .027 & .450 & .166 \\
\hline 91 & 42 & .028 & .448 & .167 \\
\hline 92 & 29 & .073 & .212 & .271 \\
\hline 93 & 67 & .026 & .463 & .161 \\
\hline 94 & 33 & .004 & .770 & -.064 \\
\hline 95 & 36 & .089 & .167 & .298 \\
\hline 96 & 90 & .007 & .700 & -.085 \\
\hline 97 & 49 & .028 & .444 & -.168 \\
\hline 98 & 2 & .010 & .656 & .098 \\
\hline 99 & 91 & .013 & .611 & -.112 \\
\hline 100 & 11 & .004 & .767 & -.065 \\
\hline
\end{tabular}


Tabelle 20: Ergebnisse der linearen Regressionen für die gesamte Stichprobe für das STAI.

\begin{tabular}{|c|c|c|c|c|}
\hline Rang & PQS-Code & $\mathbf{R}^{2}$ & $\mathbf{p}$ & 及 \\
\hline 1 & 3 & .037 & .224 & .192 \\
\hline 2 & 69 & .005 & .670 & .068 \\
\hline 3 & 65 & .002 & .767 & -.047 \\
\hline 4 & 23 & .000 & .940 & .012 \\
\hline 5 & 88 & .000 & .992 & .002 \\
\hline 6 & 80 & .000 & .933 & -.013 \\
\hline 7 & 46 & .002 & .789 & .043 \\
\hline 8 & 30 & .002 & .797 & .041 \\
\hline 9 & 35 & .008 & .562 & .092 \\
\hline 10 & 54 & .000 & .924 & .015 \\
\hline 91 & 67 & .031 & .261 & -.177 \\
\hline 92 & 42 & .010 & .537 & .098 \\
\hline 93 & 33 & .106 & .035 & -.326 \\
\hline 94 & 29 & .004 & .702 & -.061 \\
\hline 95 & 36 & .008 & .562 & -.092 \\
\hline 96 & 49 & .040 & .203 & -.200 \\
\hline 97 & 90 & .001 & .814 & -.037 \\
\hline 98 & 91 & .045 & .177 & -.212 \\
\hline 99 & 2 & .035 & .237 & .186 \\
\hline 100 & 11 & .022 & .346 & -.149 \\
\hline
\end{tabular}

Tabelle 21: Ergebnisse der linearen Regressionen für die psychodynamisch behandelte Stichprobe für das STAI.

\begin{tabular}{|c|c|c|c|c|}
\hline Rang & PQS-Code & $\mathbf{R}^{\mathbf{2}}$ & $\mathbf{p}$ & 及 \\
\hline 1 & 65 & .182 & 068 & -.427 \\
\hline 2 & 3 & .019 & .571 & .139 \\
\hline 3 & 69 & .074 & .261 & .271 \\
\hline 4 & 23 & .008 & .724 & .087 \\
\hline 5 & 80 & .073 & .262 & -.271 \\
\hline 6 & 88 & .085 & .227 & -.291 \\
\hline 7 & 35 & .029 & .487 & .170 \\
\hline 8 & 6 & .033 & .453 & -.183 \\
\hline 9 & 46 & .012 & .659 & .108 \\
\hline 10 & 54 & .077 & .250 & .277 \\
\hline 91 & 33 & .090 & .212 & -.300 \\
\hline 92 & 38 & .165 & .084 & .406 \\
\hline 93 & 42 & .042 & .398 & .206 \\
\hline 94 & 29 & .037 & .428 & -.193 \\
\hline 95 & 36 & .003 & .837 & -.050 \\
\hline 96 & 49 & .018 & .580 & -.136 \\
\hline 97 & 90 & .001 & .915 & .026 \\
\hline 98 & 91 & .136 & .120 & -.369 \\
\hline 99 & 11 & .007 & .730 & -.085 \\
\hline 100 & 2 & .052 & .349 & .227 \\
\hline
\end{tabular}


Tabelle 22: Ergebnisse der linearen Regressionen für die kognitiv-behavioral behandelte Stichprobe für das STAI.

\begin{tabular}{|c|c|c|c|c|}
\hline Rang & PQS-Code & $\mathbf{R}^{\mathbf{2}}$ & $\mathbf{p}$ & 及 \\
\hline 1 & 3 & .012 & .621 & .109 \\
\hline 2 & 69 & .041 & .356 & -.202 \\
\hline 3 & 23 & .018 & .537 & -.136 \\
\hline 4 & 88 & .018 & .547 & .132 \\
\hline 5 & 65 & .063 & .247 & .251 \\
\hline 6 & 30 & .159 & .060 & -.398 \\
\hline 7 & 46 & .048 & .316 & -.219 \\
\hline 8 & 80 & .018 & .541 & .134 \\
\hline 9 & 45 & .002 & .836 & .046 \\
\hline 10 & 72 & .002 & .833 & .046 \\
\hline 91 & 42 & .004 & .779 & .062 \\
\hline 92 & 29 & .004 & .765 & .066 \\
\hline 93 & 67 & .107 & .128 & .327 \\
\hline 94 & 33 & .103 & .135 & -.321 \\
\hline 95 & 36 & .028 & .447 & .167 \\
\hline 96 & 90 & .000 & .959 & -.011 \\
\hline 97 & 49 & .000 & .920 & -.022 \\
\hline 98 & 2 & .008 & .677 & .092 \\
\hline 99 & 91 & .014 & .596 & .117 \\
\hline 100 & 11 & .020 & .522 & -.141 \\
\hline
\end{tabular}

Tabelle 23: Ergebnisse der linearen Regressionen für die gesamte Stichprobe für den PSWQ.

\begin{tabular}{|c|c|c|c|c|}
\hline Rang & PQS-Code & $\mathbf{R}^{\mathbf{2}}$ & $\mathbf{p}$ & 及 \\
\hline 1 & 3 & .089 & .055 & .298 \\
\hline 2 & 69 & .014 & .463 & .116 \\
\hline 3 & 65 & .003 & .717 & .058 \\
\hline 4 & 23 & .002 & .774 & .046 \\
\hline 5 & 88 & .014 & .451 & .119 \\
\hline 6 & 80 & .030 & .270 & -.174 \\
\hline 7 & 46 & .003 & .725 & .056 \\
\hline 8 & 30 & .010 & .538 & -.098 \\
\hline 9 & 35 & .016 & .428 & -.126 \\
\hline 10 & 54 & .026 & .310 & .161 \\
\hline 91 & 67 & .072 & .086 & -.268 \\
\hline 92 & 42 & .054 & .139 & .232 \\
\hline 93 & 33 & .065 & .103 & -.255 \\
\hline 94 & 29 & .014 & .457 & .118 \\
\hline 95 & 36 & .026 & .312 & -.160 \\
\hline 96 & 49 & .035 & .236 & -.187 \\
\hline 97 & 90 & .126 & .021 & -.355 \\
\hline 98 & 91 & .098 & .043 & -.314 \\
\hline 99 & 2 & .005 & .653 & .071 \\
\hline 100 & 11 & .055 & .136 & -.234 \\
\hline
\end{tabular}


Tabelle 24: Ergebnisse der linearen Regressionen für die kognitiv-behavioral behandelte Stichprobe für den PSWQ.

\begin{tabular}{|c|c|c|c|c|}
\hline Rang & PQS-Code & $\mathbf{R}^{2}$ & $\mathbf{p}$ & 及 \\
\hline 1 & 3 & .120 & .106 & .346 \\
\hline 2 & 69 & .011 & .627 & -.107 \\
\hline 3 & 23 & .012 & .616 & .111 \\
\hline 4 & 88 & .074 & .208 & .273 \\
\hline 5 & 65 & .146 & .072 & .381 \\
\hline 6 & 30 & .138 & .081 & -.372 \\
\hline 7 & 46 & .052 & .295 & -.228 \\
\hline 8 & 80 & .021 & .505 & -.146 \\
\hline 9 & 45 & .004 & .776 & -.063 \\
\hline 10 & 72 & .023 & .486 & .153 \\
\hline 91 & 42 & .098 & .147 & .312 \\
\hline 92 & 29 & .038 & .376 & .194 \\
\hline 93 & 67 & .053 & .290 & .230 \\
\hline 94 & 33 & .030 & .431 & -.172 \\
\hline 95 & 36 & .023 & .491 & .151 \\
\hline 96 & 90 & .001 & .906 & -.026 \\
\hline 97 & 49 & .011 & .636 & .104 \\
\hline 98 & 2 & .018 & .547 & -.132 \\
\hline 99 & 91 & .052 & .294 & -.229 \\
\hline 100 & 11 & .155 & .064 & -.393 \\
\hline
\end{tabular}

Tabelle 25: Ergebnisse der linearen Regressionen für die psychodynamisch behandelte Stichprobe für den PSWQ.

\begin{tabular}{|c|c|c|c|c|}
\hline Rang & PQS-Code & $\mathbf{R}^{\mathbf{2}}$ & $\mathbf{p}$ & 及 \\
\hline 1 & 65 & .037 & .430 & -.192 \\
\hline 2 & 3 & .012 & .661 & .108 \\
\hline 3 & 69 & .071 & .269 & .267 \\
\hline 4 & 23 & .010 & .680 & -.101 \\
\hline 5 & 80 & .104 & .178 & .322 \\
\hline 6 & 88 & .051 & .354 & -.225 \\
\hline 7 & 35 & .013 & .637 & -.116 \\
\hline 8 & 6 & .012 & .652 & -.111 \\
\hline 9 & 46 & .000 & .966 & .011 \\
\hline 10 & 54 & .000 & .972 & .009 \\
\hline 91 & 33 & .056 & .331 & -.236 \\
\hline 92 & 38 & .095 & .198 & .309 \\
\hline 93 & 42 & .087 & .221 & .295 \\
\hline 94 & 29 & .011 & .667 & .106 \\
\hline 95 & 36 & .015 & .612 & -.124 \\
\hline 96 & 49 & .010 & .683 & -.100 \\
\hline 97 & 90 & .406 & .003 & -.637 \\
\hline 98 & 91 & .152 & .098 & -.391 \\
\hline 99 & 11 & .022 & .545 & -.148 \\
\hline 100 & 2 & .082 & .234 & .287 \\
\hline
\end{tabular}


Anhang B

Tabelle 26: Ergebnisse der linearen Regressionen für die gesamte Stichprobe für das IIP.

\begin{tabular}{|c|c|c|c|c|}
\hline Rang & PQS-Code & $\mathbf{R}^{2}$ & $\mathbf{p}$ & 及 \\
\hline 1 & 3 & .003 & .731 & .055 \\
\hline 2 & 69 & .002 & .768 & -.047 \\
\hline 3 & 65 & .029 & .283 & -.169 \\
\hline 4 & 23 & .014 & .451 & -.120 \\
\hline 5 & 88 & .007 & .587 & .086 \\
\hline 6 & 80 & .020 & .373 & .141 \\
\hline 7 & 46 & .005 & .668 & -.068 \\
\hline 8 & 30 & .004 & .682 & -.065 \\
\hline 9 & 35 & .000 & 1,0 & .000 \\
\hline 10 & 54 & .004 & .680 & -.066 \\
\hline 91 & 67 & .000 & .956 & -.009 \\
\hline 92 & 42 & .016 & .432 & .125 \\
\hline 93 & 33 & .000 & .959 & .008 \\
\hline 94 & 29 & .017 & .406 & -.132 \\
\hline 95 & 36 & .036 & .227 & -.190 \\
\hline 96 & 49 & .089 & .054 & -.299 \\
\hline 97 & 90 & .016 & .424 & -.127 \\
\hline 98 & 91 & .007 & .592 & .085 \\
\hline 99 & 2 & .034 & .245 & -.183 \\
\hline 100 & 11 & .011 & .518 & .103 \\
\hline
\end{tabular}

Tabelle 27: Ergebnisse der linearen Regressionen für die kognitiv-behavioral behandelte Stichprobe für das IIP.

\begin{tabular}{|c|c|c|c|c|}
\hline Rang & PQS-Code & $\mathbf{R}^{\mathbf{2}}$ & $\mathbf{p}$ & $\mathbf{\beta}$ \\
\hline 1 & 3 & .056 & .277 & -.237 \\
\hline 2 & 69 & .003 & .810 & -.053 \\
\hline 3 & 23 & .003 & .808 & -.054 \\
\hline 4 & 88 & .038 & .372 & .195 \\
\hline 5 & 65 & .041 & .356 & -.202 \\
\hline 6 & 30 & .038 & .371 & -.196 \\
\hline 7 & 46 & .085 & .177 & -.291 \\
\hline 8 & 80 & .000 & .983 & -.005 \\
\hline 9 & 45 & .005 & .751 & .070 \\
\hline 10 & 72 & .042 & .348 & .205 \\
\hline 91 & 42 & .014 & .597 & .116 \\
\hline 92 & 29 & .000 & .989 & .003 \\
\hline 93 & 67 & .134 & .086 & .366 \\
\hline 94 & 33 & .004 & .764 & .066 \\
\hline 95 & 36 & .001 & .901 & .028 \\
\hline 96 & 90 & .006 & .736 & .074 \\
\hline 97 & 49 & .003 & .805 & -.054 \\
\hline 98 & 2 & .022 & .499 & -.148 \\
\hline 99 & 91 & .036 & .389 & .189 \\
\hline 100 & 11 & .005 & .748 & .071 \\
\hline
\end{tabular}


Anhang B

Tabelle 28: Ergebnisse der linearen Regressionen für die psychodynamisch behandelte Stichprobe für das IIP.

\begin{tabular}{|c|c|c|c|c|}
\hline Rang & PQS-Code & $\mathbf{R}^{2}$ & P & 及 \\
\hline 1 & 65 & .009 & .693 & .097 \\
\hline 2 & 3 & .079 & .244 & .281 \\
\hline 3 & 69 & .009 & .698 & -.095 \\
\hline 4 & 23 & .053 & .341 & -.231 \\
\hline 5 & 80 & .113 & .159 & .336 \\
\hline 6 & 88 & .007 & .736 & -.083 \\
\hline 7 & 35 & .000 & .974 & -.008 \\
\hline 8 & 6 & .224 & .040 & .474 \\
\hline 9 & 46 & .052 & .346 & .229 \\
\hline 10 & 54 & .035 & .446 & -.186 \\
\hline 91 & 33 & .001 & .884 & .030 \\
\hline 92 & 38 & .074 & .261 & -.272 \\
\hline 93 & 42 & .031 & .474 & .175 \\
\hline 94 & 29 & .101 & .184 & -.319 \\
\hline 95 & 36 & .094 & .203 & -.306 \\
\hline 96 & 49 & .195 & .058 & -.441 \\
\hline 97 & 90 & .082 & .235 & -.286 \\
\hline 98 & 91 & .008 & .715 & .090 \\
\hline 99 & 11 & .054 & .339 & .232 \\
\hline 100 & 2 & .157 & .093 & -.396 \\
\hline
\end{tabular}




\section{LEBENS L A U F}

\section{Persönliche Daten}

Vor- und Zuname

Geburtstag

Geburtsort

Wohnort

Staatsangehörigkeit

Familienstand

\section{Schulbildung}

September 1986 - September 1988

September 1988 - September 1989

September 1989 - September 1990

Oktober 1990 - Juni 1999

\section{Studium}

Oktober 1999 - November 2005

Titel der Diplomarbeit:

\section{Praktika während des Studiums}

23.09.2002-07.11.2002

29.09.2003 - 07.11.2003

1.4.2003 - 30.3.2004

März 2005 - Oktober 2005

\author{
Norina Hofmann \\ 21.02.1980 \\ Dresden \\ 37073 Göttingen \\ deutsch \\ ledig
}

77. Grundschule „An den Seegärten" in Dresden

34. Polytechnische Oberschule „Ernst Moritz Arndt" in Dresden

"Grundschule im Mühlenfeld" in Willich (NordrheinWestfalen)

„Weidig Gymnasium" in Butzbach (Hessen)

Abschluss: Abitur (Note: 1,3)

Studium der Psychologie an der

Georg-August-Universität Göttingen

Abschluss: Diplom (Note: 1,25)

„Psychologie als Frauenstudium - Eine Untersuchung über mögliche Erklärungen für das ungleiche Geschlechterverhältnis in der Psychologie"

Praktikum in der Kontakt- und Beratungsstelle für Jugendliche und junge Erwachsene

Praktikum in der Psychiatrischen Universitätsklinik Göttingen

Praktikum in der Abteilung für Klinische Psychologie und Psychotherapie des Psychologischen Instituts der Universität Göttingen

ehrenamtliche Mitarbeit im Projekt „Zeit für ein Kind“ durch Übernahme einer Patenschaft für ein kurdisches Mädchen mit Sprach- und Kontaktschwierigkeiten 


\section{Beruflicher Werdegang}

Februar - April 2006

Mai 2006 - Dezember 2007

Oktober 2006

seit Juli 2007

seit Juli 2007
Wissenschaftliche Hilfskraft am Psychologischen Institut der Universität Göttingen

Arbeit an der Dissertation („Kognitiv-behaviorale und tiefenpsychologische Therapie der Generalisierten Angst: Ein Therapieprozessvergleich.")

Beginn des Weiterbildenden Studiengangs „Psychologische Psychotherapie“ an den Universitäten Göttingen/Braunschweig

Praktische Tätigkeit in der Psychiatrie des Universitätsklinikums Göttingen („Psychologin im Praktikum")

Wissenschaftliche Hilfskraft in einem Forschungsprojekt zur psychotherapeutischen Behandlung der Sozialen Phobie 